\title{
Towards primary prevention of dementia
}

\author{
Citation for published version (APA):
}

Heger, I. S. (2022). Towards primary prevention of dementia: raising awareness for dementia risk reduction in the general population. [Doctoral Thesis, Maastricht University]. Gildeprint Drukkerijen. https://doi.org/10.26481/dis.20220324ih

Document status and date:

Published: 01/01/2022

DOI:

10.26481/dis.20220324ih

Document Version:

Publisher's PDF, also known as Version of record

\section{Please check the document version of this publication:}

- A submitted manuscript is the version of the article upon submission and before peer-review. There can be important differences between the submitted version and the official published version of record.

People interested in the research are advised to contact the author for the final version of the publication, or visit the DOI to the publisher's website.

- The final author version and the galley proof are versions of the publication after peer review.

- The final published version features the final layout of the paper including the volume, issue and page numbers.

Link to publication

\footnotetext{
General rights rights.

- You may freely distribute the URL identifying the publication in the public portal. please follow below link for the End User Agreement:

www.umlib.nl/taverne-license

Take down policy

If you believe that this document breaches copyright please contact us at:

repository@maastrichtuniversity.nl

providing details and we will investigate your claim.
}

Copyright and moral rights for the publications made accessible in the public portal are retained by the authors and/or other copyright owners and it is a condition of accessing publications that users recognise and abide by the legal requirements associated with these

- Users may download and print one copy of any publication from the public portal for the purpose of private study or research.

- You may not further distribute the material or use it for any profit-making activity or commercial gain

If the publication is distributed under the terms of Article $25 \mathrm{fa}$ of the Dutch Copyright Act, indicated by the "Taverne" license above, 


\section{Towards Primary Prevention of Dementia}

Raising awareness for dementia risk reduction in the general population

Irene Sophia Heger 
(C) Irene Sophia Heger, Maastricht, 2022

All rights reserved. No part of this book may be reproduced or transmitted in any form or by any means without permission from the first author, or where appropriate, from the publishers of the publication.

Cover design and layout: Zuiderlicht

Printed by: Gildeprint - www.gildeprint.nl

ISBN: 978-94-6423-683-5

ISBN e-book: 978-94-6423-690-3 


\title{
Towards Primary Prevention of Dementia
}

\author{
Raising awareness for dementia risk reduction \\ in the general population
}

\section{PROEFSCHRIFT}

Ter verkrijging van de graad van doctor aan de Universiteit Maastricht, op gezag van de Rector Magnificus, Prof. dr. Pamela Habibovic, volgens het besluit van het College van Decanen, in het openbaar te verdedigen op donderdag 24 maart 2022 om 16.00 uur

door

Irene Sophia Heger

Geboren op 31 oktober 1987 te Voorburg 

Promotores:

Co-promotor:

Beoordelingscommissie:
Dr. S. Köhler

Prof. dr. F. R. J. Verhey

Dr. M.P.J. van Boxtel

Prof. dr. R. Crutzen (voorzitter)

Dr. J.A.H.R Claassen (Radboudumc, Nijmegen)

Prof. dr. C.M. van Heugten

Prof. dr. J.W.M. Muris

Dr. C.H.M Smits (Pharos Expertisecentrum

gezondheidsverschillen, Utrecht)

The research described in this thesis was performed at the Department of Psychiatry and Neuropsychology, School for Mental Health and Neuroscience, Maastricht University, Alzheimer Centre Limburg, Maastricht, the Netherlands.

The research presented in this thesis was supported by the Ministry of Economic Affairs by means of the PPP Allowance made available by the Top Sector Life Sciences \& Health to stimulate public-private partnerships (LSHM17080-SGF); the Province of Limburg (SAS-2015-04931); and Health Foundation Limburg.

Printing of this thesis was kindly supported by Alzheimer Nederland and Maastricht University. 



\section{Contents}

Chapter $1 \quad$ General introduction, thesis aim and outline

\begin{tabular}{lll}
\hline Part $1 \quad$ Epidemiological perspective & 25
\end{tabular}

Chapter 2 Associations of the LIfestyle for BRAin Health (LIBRA) index

with structural brain changes and cognition: results from The

Maastricht Study

Neurology, 2021

Chapter 3 Socioeconomic position, modifiable dementia risk and cognitive decline: results of 12-year the Maastricht Aging Study

International Journal of Epidemiology, submitted

\begin{tabular}{|c|c|c|}
\hline Part 2 & Public health perspective & 97 \\
\hline Chapter 4 & $\begin{array}{l}\text { Dementia awareness and risk perception in middle-aged and older } \\
\text { individuals: baseline results of the MijnBreincoach survey on the } \\
\text { association between lifestyle and brain health } \\
\text { BMC Public Health, } 2019\end{array}$ & 99 \\
\hline Chapter 5 & $\begin{array}{l}\text { Raising awareness for dementia risk reduction through a public } \\
\text { health campaign: a pre-post study } \\
\text { BMJ Open, } 2020\end{array}$ & 131 \\
\hline Chapter 6 & $\begin{array}{l}\text { Appreciation of an mHealth tool to increase knowledge and beliefs } \\
\text { and attitudes for dementia risk reduction: a pre-post proof-of- } \\
\text { concept study } \\
\text { Journal of Medical Internet Research mHealth and uHealth, } \\
\text { pending revisions }\end{array}$ & 155 \\
\hline Chapter 7 & General discussion & 195 \\
\hline \multirow[t]{8}{*}{ Addendum } & & 211 \\
\hline & Summary & 213 \\
\hline & Nederlandse samenvatting & 219 \\
\hline & Impact paragraph & 225 \\
\hline & List of publications & 233 \\
\hline & Dankwoord & 239 \\
\hline & Thesis defences from MHeNs & 247 \\
\hline & Author information & 255 \\
\hline
\end{tabular}



Chapter 1

General introduction, thesis aim and outline

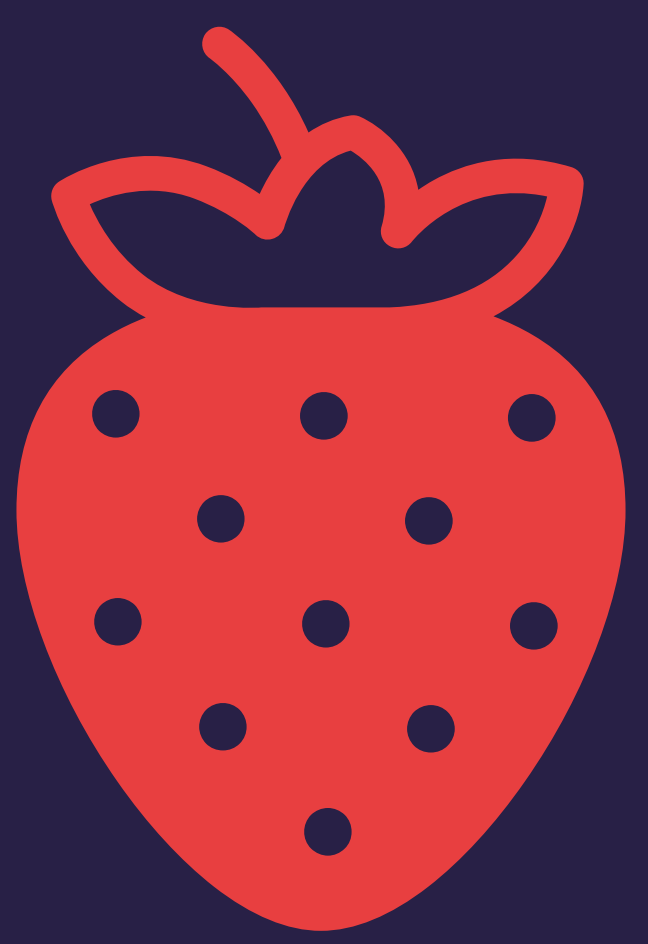





\section{Introduction}

Like in most other non-communicable diseases such as cancer and chronic lung disease $^{1}$, a part of the dementia risk is modifiable. Latest estimates suggest that around $40 \%$ of all dementia cases worldwide are potentially preventable through lifestyle change and proper cardiovascular risk management ${ }^{2}$. While these insights are promising, awareness of dementia risk reduction in the general population is still low and most people still perceive dementia as an inevitable part of aging ${ }^{3}$. This General Introduction will place this thesis in the context of national and international dementia research. It defines what dementia is, including the current state of research on dementia risk reduction, awareness in the general population, and how this thesis aims to contribute to the field.

\section{Dementia}

Dementia is a syndrome with a wide variety of clinical manifestations and aetiologies. While mild decline in cognitive performance can be part of normal aging, the cognitive deficits in dementia are more substantial, most often progressive, interfere with independence in everyday activities and are caused by underlying neuropathology such as in Alzheimer's disease and vascular dementia ${ }^{4,5}$. The number of people with dementia is rising worldwide due to our aging population, as age is the most important risk factor. In the Netherlands, dementia prevalence has increased six-fold from 1950 to 2021, with estimates showing that the current 290.000 people with dementia in the Netherlands will increase to 620.000 in $2050^{6}$. Dementia has a substantial impact on the person with the diagnosis, relatives and carers, as well as on the society and economy as a whole ${ }^{4-6}$. To date, there is no curative treatment available for dementia with clinically relevant health benefits ${ }^{4,5}$.

\section{The potential of prevention}

Recent trends in dementia rates provide better understanding into the topic of dementia risk reduction and the potential of prevention. Epidemiological studies have recently shown that the age-specific prevalence and incidence of dementia are stable or even declining in high-income countries, probably due to improvements in vascular health management, risk factor control, and improved provision of and access to education and health care. In contrast, an increasing number of people in low-to-middle income countries are at increased risk for developing dementia, reflected by the increased presence of risk factors (e.g., diabetes, hypertension, and smoking), less health care opportunities, lower 
risk awareness and less educational attainment ${ }^{7-12}$. An important underlying concept in understanding the protective effect of educational attainment and other cognitively stimulating activities is cognitive reserve ${ }^{13}$. Cognitive reserve explains the differences between individuals in level of tolerance to age-related brain changes. Some people seem less susceptible to these brain changes and can still maintain their cognitive function, compared to others. It appears that this level of tolerance -the cognitive reserve- can be increased by engaging in cognitively stimulating activities, leading to a delay in cognitive decline and dementia onset ${ }^{13}$.

Above all, the diverging trend in dementia rates between high- and low-tomiddle income countries shows the worldwide health inequalities that play a significant role in health status and incidence of diseases. It also shows the potential for primary prevention of dementia. Despite unmodifiable factors, such as genetics and age, there seems to be room to improve brain health and delay or prevent dementia onset via improved general health, lifestyle and wellbeing.

\section{Recent developments in dementia research and policy}

The life-course model of the Lancet Commission on Dementia Prevention, Intervention and $\mathrm{Care}^{14}$ has been updated in 2020 and its authors conclude that around $40 \%$ of dementia is potentially attributable to 12 modifiable risk factors. Risk factors vary across the life span, from less education in early life, hypertension and excessive alcohol consumption in midlife, and smoking, depression and physical inactivity in later life. One of the key messages of the Commission is to "be ambitious about prevention"2. To scaffold these associations between modifiable risk factors and dementia as being causal in nature, randomized controlled trials are needed. The 2-year Finnish Geriatric Intervention Study to Prevent Cognitive Impairment and Disability (FINGER) was the first large-scale multi-domain lifestyle trial to find beneficial effects on cognition ${ }^{15}$. A recent initiative called World Wide FINGERS was set up to harmonize worldwide intervention trials aimed at delaying or preventing dementia onset ${ }^{16}$. The Dutch branch, the FINGER-NL study, was launched in 2021.

From a policy perspective, an important mark was the identification of risk reduction of dementia as one of the action areas for 2017-2025 by the World Health Organization ${ }^{17}$, followed by publishing guidelines for risk reduction of 
cognitive decline and dementia in $2019^{18}$. In the same year, 67 Dutch health professionals and scientists published a letter in a national newspaper in which they summoned the Minister of Health, Welfare and Sports to invest in primary prevention of dementia ${ }^{19}$. The National Dementia Strategy 2021-2030 was published in the following year. Overall, the publication of this Strategy seems a promising development for the Dutch dementia policy. However, dementia risk reduction is not incorporated as one of the major pillars of the strategy yet $^{20}$.

\section{Estimating the risk of dementia}

Dementia risk indices aim to quantify the dementia risk to identify at-risk individuals that can be targeted for prevention initiatives. However, most existing indices are developed within a single cohort study and have not been externally validated. This is problematic since such indices are prone to capitalizing on chance or on associations that only exist in this specific cohort $^{21}$. In addition, they usually incorporate factors that are not amenable to change, most notably age ${ }^{22-27}$. Age remains the best predictor of dementia risk and has on its own almost the same predictive validity as complete indices ${ }^{28}$. Age essentially remains a container variable, which presumably makes up a proxy measure of yet unidentified age-related disease mechanisms with an impact on cognitive outcome. In addition, age does not inform about the room for improvement. In 2015, the LIfestyle for BRAin health (LIBRA) index was developed by researchers from Maastricht University, based on a comprehensive literature review and international Delphi consensus rounds ${ }^{29}$. As shown in Figure 1, LIBRA incorporates twelve potentially modifiable factors and aims to identify at-risk individuals who will benefit most from lifestyle interventions and cardiovascular risk management. Therefore, LIBRA is considered to be useful as a participant selection tool and as a surrogate outcome measure in lifestyle intervention trials ${ }^{30}$, and to inform people about target behaviours in public health initiatives. Indeed, to date LIBRA was shown to predict cognitive decline and dementia risk in several prospective cohort studies ${ }^{31-36}$. 


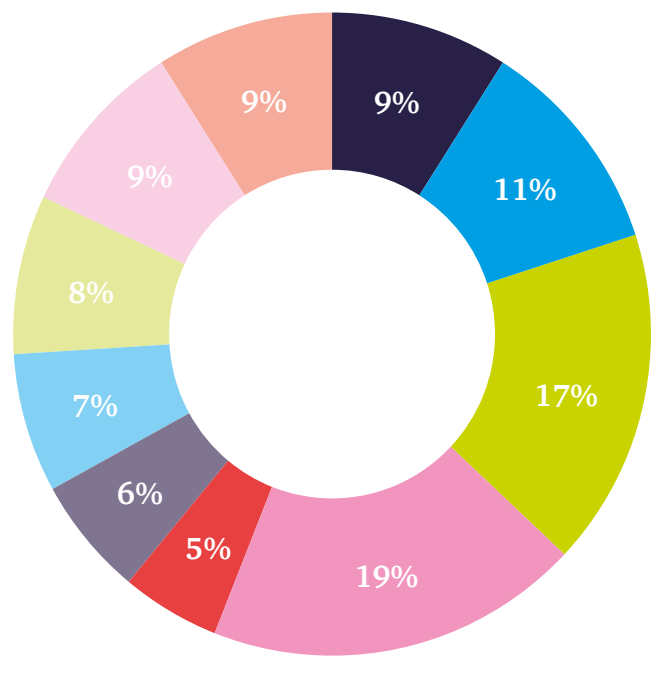

- Healthy diet/ Mediterranean diet

- Depression

- High cognitive activity

- Remember to manage:

- Chronic kidney disease 6\%

- Diabetes 7\%

- Coronary heart disease 6\%

- Low/moderate alcohol intake

- Physical inactivity

- Cholesterol

- Smoking

Midlife obesity

- Midlife hypertension

Figure 1. The individual contribution of each risk or protective factor included in the total score of the LIfestyle for BRAin health (LIBRA) index (based on weight from existing meta-analyses).

\section{Risk awareness}

From a public health perspective, these results are promising. However, most people in the general population are still unaware of the potential of dementia prevention, let alone of specific actions to reduce dementia risk. The British Social Attitudes survey from 2015 concluded that the public knowledge of dementia risk factors is considerably low. The majority of people did select dementia in their top three from a list of health conditions for clinicians and scientists to prevent ${ }^{37}$. According to a systematic review from 2018, almost half of all respondents perceived dementia as a condition which is "nonpreventable"3. Gaps of knowledge exist in particular for the cardiovascular risk factors of dementia (e.g., hypertension or obesity) which means that most people are not aware of the insight that "what is healthy for the heart, is healthy for the brain". 


\section{Thesis aims and outline}

This thesis used two different perspectives to contribute to the field of dementia risk reduction. In the first part, an epidemiological perspective was taken to further validate LIBRA. To explain underlying mechanisms of the well-established association between LIBRA and cognitive performance and dementia risk, we explored plausible biological pathways of this association, using volumetric markers in brain Magnetic Resonance Imaging (MRI). In another study, we investigated whether the association between LIBRA and rate of cognitive decline differs across different socioeconomic strata. With this study, we aimed to identify at-risk groups that are important to target and design interventions for. In the second part of this thesis, a public health perspective was chosen, in which we assessed the level of awareness of dementia risk reduction in the general population and conducted proof-ofconcept studies aimed at raising awareness of dementia risk reduction in the general population. For this purpose, a public health campaign ("We zijn zelf het medicijn") and mHealth intervention ("MijnBreincoach" or "MyBraincoach") were developed and evaluated. LIBRA was used to identify individual roomfor-lifestyle-improvement and to motivate people to make brain-healthy lifestyle choices. The underlying theoretical behavioural change model for these proof-of-concept studies was the Theory of Planned Behaviour (see Figure 2). In short, this theory assumes that behavioural beliefs (i.e., the likely consequences of the behaviour) determine the attitudes toward the behaviour, normative beliefs (i.e., expectations from others) determine the subjective norm (i.e., the perceived social pressure) and control beliefs (i.e., facilitators and barriers to engage in the behaviour) determine the perceived behavioural control $^{38,39}$. These constructs, subsequently, influence the intention to perform the behaviour. The intention and the behavioural control, in turn, predict the actual behaviour ${ }^{38,39}$. We aimed to identify both the barriers and difficulties in developing and executing public health initiatives for raising awareness of dementia risk reduction, as well as the advantages and gains, to improve our understanding of key factors that need to be considered in future studies. Also, we aimed to facilitate steps towards joining (inter)national forces towards primary prevention of dementia. 


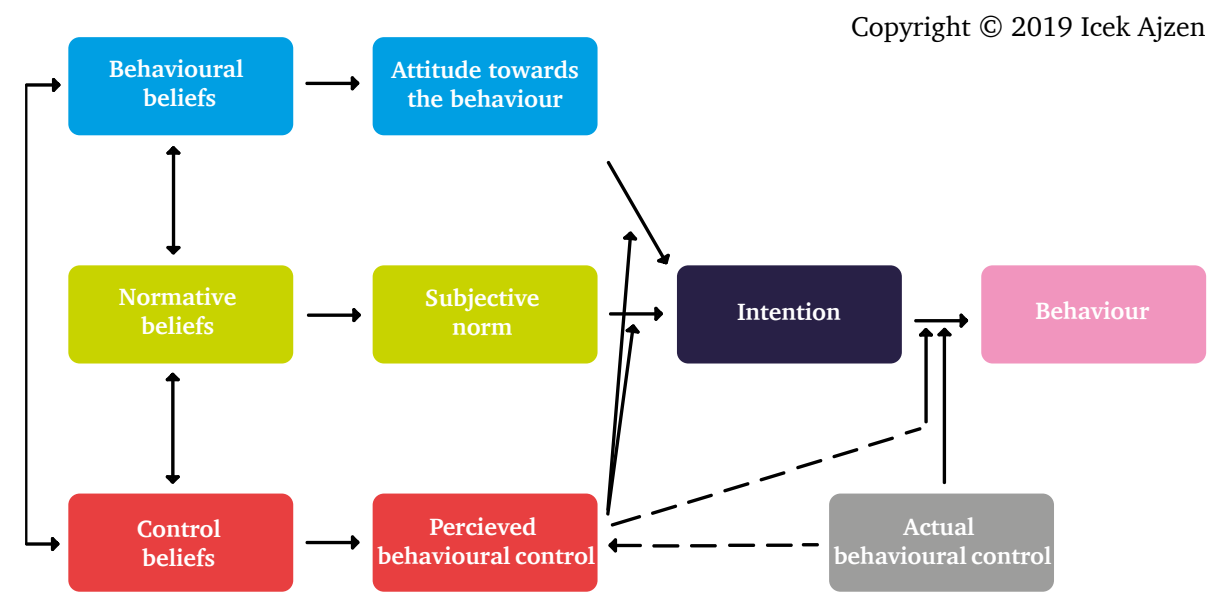

Figure 2. Diagram of the different constructs of the Theory of Planned Behaviour. 
The outline of this thesis is as follows:

\section{Part 1: Epidemiological perspective}

Chapter 2 addressed the following research question: To what extent can volumetric brain markers explain the association between LIBRA and cognitive performance? This question was addressed using data of The Maastricht Study, a population-based cohort study in the south of Limburg, the Netherlands. A cross-sectional analysis was conducted to analyse the indirect, mediating effect of volumetric brain markers in the association between LIBRA and cognitive performance (including memory function, information processing speed and executive functioning and attention).

The role of socioeconomic status was the focus of Chapter 3, with the following research question: Are there socioeconomic differences in the relationship between LIBRA and cognitive functioning over time? We analysed this question using the 12-year follow-up of the Maastricht Aging Study (MAAS), an ongoing prospective cohort study in the province of Limburg, the Netherlands.

\section{Part 2: Public health perspective}

Chapter 4 focussed on assessing literacy of dementia risk reduction in a random sample of middle-aged community-dwelling individuals in the province of Limburg. We aimed to answer the following research question: What does the general public know about modifiable dementia risk and protective factors and what are their needs, wishes and barriers to engage in a brain-healthy lifestyle? This study was used as the rationale for the developed public health initiatives that are described in Chapter 5 and Chapter 6.

Chapter 5 described and evaluated a 10-month public health campaign that was launched in the Province of Limburg, the Netherlands, aimed at raising awareness of dementia risk reduction. This chapter focussed on the research question: What is the effect of a public health campaign on level of awareness of dementia risk reduction and what are the lessons learned for future studies and public health initiatives? The assessment that was described in Chapter 4 was repeated in a new random sample after the campaign to investigate pre/post-campaign differences in level of awareness. 
Chapter 6 focussed on the MijnBreincoach mHealth intervention with the research question: To what extent is the MijnBreincoach app used and appreciated, and what is the effect of the app on perceived knowledge and beliefs and attitudes towards dementia risk reduction? A proof-of-concept study was carried out in which two versions of the tool were used, which differed on the level of personalization ("tailoring").

The last Chapter 7 provided a general discussion of the main finding of this thesis, including the methodological considerations, implications, and future directions. 


\section{References}

1. World Health Organization. Preventing noncommunicable diseases. https:// www.who.int/activities/preventingnoncommunicable-diseases (14-04-2021, date last accessed).

2. Livingston G, Huntley J, Sommerlad A, et al. Dementia prevention, intervention, and care: 2020 report of the Lancet Commission. Lancet 2020;396:413-46.

3. Cations M, Radisic G, Crotty M, Laver KE. What does the general public understand about prevention and treatment of dementia? A systematic review of population-based surveys. PLoS One 2018;13.

4. Patterson C, World Alzheimer Report 2018, in The state of the art of dementia research: New frontiers. 2018, Alzheimer's Disease International: London.

5. World Health Organization. Dementia. 2020. https://www.who.int/news-room/ fact-sheets/detail/dementia (14-04-2021, date last accessed).

6. Alzheimer Nederland. Feiten over dementie. 2017. https://www.alzheimernederland.nl/dementie/feiten-cijfers (19 Jan, date last accessed).

7. Hachinski V, Einhäupl K, Ganten D, et al. Preventing dementia by preventing stroke: The Berlin Manifesto. Alzheimers Dement 2019;15:961-84.

8. Roehr S, Pabst A, Luck T, Riedel-Heller SG. Is dementia incidence declining in high-income countries? A systematic review and meta-analysis. Clin Epidemiol 2018;10:1233-47.
9. Prince $\mathrm{M}$, Ali G-C, Guerchet M, Prina AM, Albanese E, Wu Y-T. Recent global trends in the prevalence and incidence of dementia, and survival with dementia. Alzheimers Res Ther 2016;8:23-23.

10. Satizabal CL, Beiser AS, Chouraki V, Chêne G, Dufouil C, Seshadri S. Incidence of Dementia over Three Decades in the Framingham Heart Study. NEJM 2016;374:523-32.

11. Wolters FJ, Chibnik LB, Waziry R, et al. Twenty-seven-year time trends in dementia incidence in Europe and the United States: The Alzheimer Cohorts Consortium. Neurology 2020;95:e519-e31.

12. Prince $M$, Albanese E, Guerchet M, Prina AM, World Alzheimer Report 2014. Dementia and risk reduction. An analysis of protective and modifiable factors. 2014, Alzheimer's Disease International (ADI): London.

13. Stern Y. Cognitive reserve in ageing and Alzheimer's disease. Lancet Neurol 2012;11:1006-12.

14. Livingston G, Sommerlad A, Orgeta V, et al. Dementia prevention, intervention, and care. The Lancet 2017;390:2673734.

15. Ngandu T, Lehtisalo J, Solomon A, et al. A 2 year multidomain intervention of diet, exercise, cognitive training, and vascular risk monitoring versus control to prevent cognitive decline in at-risk elderly people (FINGER): a randomised controlled trial. Lancet 2015;385:2255-63.

16. Kivipelto M, Mangialasche F, Snyder HM, 
et al. World-Wide FINGERS Network: A global approach to risk reduction and prevention of dementia. Alzheimers Dement 2020;16:1078-94.

17. Global action plan on the public health response to dementia 2017-2025. 2017, World Health Organization: Geneva.

18. Risk reduction of cognitive decline and dementia: WHO guidelines. 2019, World Health Organization: Geneva.

19. Laten we de duurste ziekte aanpakken - dementie, in NRC Handelsblad. 2019: Amsterdam.

20. Nationale dementiestrategie. 2020, Ministerie van Volksgezondheid, Welzijn en Sport.

21. Tang EY, Harrison SL, Errington $\mathrm{L}$, et al. Current Developments in Dementia Risk Prediction Modelling: An Updated Systematic Review. PLoS One 2015;10:e0136181.

22. Stephen R, Liu $Y$, Ngandu $T$, et al. Associations of CAIDE Dementia Risk Score with MRI, PIB-PET measures, and cognition. J Alzheimers Dis 2017;59:695705.

23. Kivipelto $M$, Ngandu $T$, Laatikainen $T$, Winblad B, Soininen H, Tuomilehto J. Risk score for the prediction of dementia risk in 20 years among middle aged people: a longitudinal, population-based study. Lancet Neurol 2006;5:735-41.

24. Reitz C, Tang MX, Schupf N, Manly JJ, Mayeux R, Luchsinger JA. A summary risk score for the prediction of Alzheimer disease in elderly persons. Arch Neurol 2010;67:835-41.

25. Exalto LG, Quesenberry CP, Barnes D, Kivipelto M, Biessels GJ, Whitmer RA.
Midlife risk score for the prediction of dementia four decades later. Alzheimers Dement 2014;10:562-70.

26. Cherbuin N, Shaw ME, Walsh E, Sachdev P, Anstey KJ. Validated Alzheimer's Disease Risk Index (ANU-ADRI) is associated with smaller volumes in the default mode network in the early 60s. Brain Imaging Behav 2019;13:65-74.

27. Barnes DE, Yaffe K. Predicting dementia: role of dementia risk indices. Future Neurol 2009;4:555-60.

28. Licher S, Yilmaz , Leening MJG, et al. External validation of four dementia prediction models for use in the general community-dwelling population: a comparative analysis from the Rotterdam Study. Eur J Epidemiol 2018;33:645-55.

29. Deckers K, van Boxtel MP, Schiepers OJ, et al. Target risk factors for dementia prevention: a systematic review and Delphi consensus study on the evidence from observational studies. Int $J$ Geriatr Psychiatry 2015;30:234-46.

30. Coley N, Hoevenaar-Blom MP, van Dalen JW, et al. Dementia risk scores as surrogate outcomes for lifestyle-based multidomain prevention trials-rationale, preliminary evidence and chal lenges. Alzheimers Dement 2020;16:1674-85.

31. Vos SJB, van Boxtel MPJ, Schiepers OJG, et al. Modifiable Risk Factors for Prevention of Dementia in Midlife, Late Life and the Oldest-Old: Validation of the LIBRA Index. $J$ Alzheimers Dis 2017;58:537-47.

32. Schiepers OJG, Kohler S, Deckers K, et al. Lifestyle for Brain Health (LIBRA): a 
new model for dementia prevention. Int $J$ 1996;11:87-98.

Geriatr Psychiatry 2018;33:167-75.

33. Pons A, LaMonica HM, Mowszowski L, Kohler S, Deckers K, Naismith SL. Utility of the LIBRA Index in Relation to Cognitive Functioning in a Clinical Health Seeking Sample. J Alzheimers Dis 2018;62:373-84.

34. Deckers K, Nooyens A, van Boxtel M, Verhey F, Verschuren M, Kohler S. Gender and Educational Differences in the Association between Lifestyle and Cognitive Decline over 10 Years: The Doetinchem Cohort Study. $J$ Alzheimers Dis 2018;70:S31-S41.

35. Deckers K, Cadar D, van Boxtel MPJ, Verhey FRJ, Steptoe A, Kohler S. Modifiable Risk Factors Explain Socioeconomic Inequalities in Dementia Risk: Evidence from a Population-Based Prospective Cohort Study. J Alzheimers Dis 2019;71:549-57.

36. Deckers $\mathrm{K}$, Köhler $\mathrm{S}$, Ngandu $\mathrm{T}$, et al. Quantifying dementia prevention potential in the FINGER randomized controlled trial using the LIBRA prevention index. Alzheimers Dement 2021.

37. Marcinkiewicz A, Reid S, Attitudes to dementia: Findings from the 2016 British Social Attitudes survey 2016, NatCen Social Research: London.

38. Ajzen I. Theory of Planned Behavior. https://people.umass.edu/aizen/index. html (May 11, 2021, date last accessed).

39. Godin G, Kok G. The theory of planned behavior: a review of its applications to health-related behaviors. AJHP 

Part 1

Epidemiological

perspective 



\section{Chapter 2}

\section{Associations of the Llfestyle for BRAin Health (LIBRA)}

\section{index with structural brain}

\section{changes and cognition: results from The Maastricht Study}

Irene Heger, Kay Deckers, Miranda Schram, Coen Stehouwer, Nicolaas Schaper, Pieter Dagnelie, Carla van der Kallen, Annemarie Koster, Simone Eussen, Jacobus Jansen, Frans Verhey, Martin van Boxtel, Sebastian Köhler 



\section{Abstract}

Background and Objectives: Observational research has shown that a substantial proportion of all dementia cases worldwide is attributable to modifiable risk factors. Dementia risk scores might be useful to identify highrisk individuals and monitor treatment adherence. The objective of this study was to investigate whether a dementia risk score, the LIfestyle for BRAin health (LIBRA) index, is associated with MRI markers and cognitive functioning/ impairment in the general population.

Methods: Cross-sectional data was used from the observational populationbased cohort of The Maastricht Study. The weighted compound score of LIBRA (including twelve dementia risk and protective factors, e.g., hypertension, physical inactivity) was calculated, with higher scores indicating higher dementia risk. Standardized volumes of white matter, grey matter, CSF (as proxy for general brain atrophy), white matter hyperintensities, and presence of cerebral small vessel disease were derived from 3T MRI. Cognitive functioning was tested in three domains: memory, information processing speed, and executive function and attention. Values $\leq 1.5$ SD below the average were defined as cognitive impairment. Multiple regression analyses and structural equation modelling were used, adjusted for age, sex, education, intracranial volume and type-2 diabetes.

Results: Participants ( $n=4,164$; mean age 59y; 49.7\% men) with higher LIBRA scores (mean $=1.19$, range $=-2.7$ to +9.2 ), denoting higher dementia risk, had higher volumes of white matter hyperintensities $(\beta=0.051, p=.002)$, and lower scores on information processing speed $(\beta=-0.067, p=.001)$ and executive function and attention $(\beta=-0.065, p=.004)$. Only in men, associations between LIBRA and volumes of grey matter $(\beta=-0.093, p<.001), \operatorname{CSF}(\beta=0.104, p<.001)$ and memory $(\beta=-0.054, p=.026)$ were found. White matter hyperintensities and CSF volume partly mediated the association between LIBRA and cognition.

Discussion: Higher health- and lifestyle-based dementia risk is associated with markers of general brain atrophy, cerebrovascular pathology and worse cognition, suggesting that LIBRA meaningfully summarizes individual lifestylerelated brain health. Improving LIBRA factors on an individual level might improve population brain health. Sex differences in lifestyle-related pathology and cognition need to be further explored. 
Classification of Evidence: This study provides Class II evidence that higher LIBRA scores are significantly associated with lower scores on some cognitive domains and a higher risk of cognitive impairment. 


\section{Introduction}

A substantial proportion of dementia cases might be attributable to modifiable risk factors ${ }^{1,2}$. Early detection of individuals at risk, allowing for timely management, has great public health implications ${ }^{1}$, as echoed by recent reports of the Lancet Commission on Dementia Prevention, Intervention and $\mathrm{Care}^{2}$ and the World Health Organization (WHO) ${ }^{3}$.

Dementia risk scores, summarizing individual risks, might be useful for selection of high-risk individuals and could serve as intermediate outcomes to monitor treatment adherence. Some risk scores have been associated with structural brain changes and cognitive functioning ${ }^{4-7}$, but most are based on single cohort studies and/or include factors that are not amenable to change, e.g., age $\mathrm{e}^{4.8}$, known to be highly correlated with brain markers. The LIfestyle for BRAin health (LIBRA) index is based on a systematic literature review and Delphi consensus on factors amendable to change ${ }^{9}$, thereby summarizing one's potential for brain health improvement ${ }^{9}$. Criterion validity has been established by several prospective studies relating higher LIBRA scores with steeper cognitive decline, incident cognitive impairment and dementia in mid- and late life ${ }^{9-14}$, and intervention effects in multifactorial randomized controlled trials ${ }^{15}$. Whether LIBRA is also related to brain markers, reflecting more direct neurobiological markers of 'brain health', remains to be elucidated.

Therefore, this study aimed to examine the association of LIBRA with cognitive performance and impairment, and evidence of neuroimaging abnormalities in the general adult population (aged 40-75 years). In addition, we investigated biological plausible pathways by testing whether MRI markers mediated the association of LIBRA with cognition. 


\section{Methods}

\section{Participants}

Data were used from The Maastricht Study, an observational population-based cohort study, of which the rationale and methodology has been described previously ${ }^{16}$. In brief, the study focusses on the etiology, pathophysiology, complications and comorbidities of type 2 diabetes mellitus (T2DM) and is characterized by an extensive phenotyping approach. Eligible for participation were individuals aged between 40 and 75 years and living in the southern part of the Netherlands. Participants were recruited through mass media campaigns and from the municipal registries and the regional Diabetes Patient Registry (which includes virtually all T2DM individuals in primary, secondary or tertiary care in the targeted population) via mailings. Recruitment was stratified according to known T2DM status, with an oversampling of individuals with T2DM, for reasons of efficiency, while at the same time monitoring the representation of the source population continuously (reprinted with permission) ${ }^{16,17}$. The present report addresses the following primary research questions: Are higher (i.e., more unhealthy) LIBRA scores associated with lower scores on cognitive functioning and a higher odds of cognitive impairment (Class II Evidence)? Are higher LIBRA scores associated with lower volumes of MRI markers and a higher odds of cerebral small vessel disease (Class II Evidence)? To what extent can volumetric MRI markers explain the association between LIBRA and cognitive functioning (Class II Evidence)? Cross-sectional data was used from participants who completed the baseline survey between November 2010 and January 2018. The examinations of each participant were performed within a time window of three months. MRI measurements were implemented from December 2013 onwards. Participants were included in the analyses if data on MRI outcomes, at least 11 LIBRA factors (see Table 1 and Operationalization of the LIBRA score) and cognition were available.

\section{Operationalization of the LIBRA score}

The individual LIBRA factors were created based on clinical data from physical examination and/or self-reported questionnaires from the baseline measurement of The Maastricht Study and then dichotomized (presence of LIBRA factor yes/no) according to established cut-offs. The LIBRA total score is computed by assigning a weight (positive for presence of risk factors; negative for presence of protective factors) to each factor, based on the relative risks from published meta-analyses ${ }^{9,18}$. Weights are then standardized and summed 
up to a total score. A higher LIBRA score reflects higher dementia risk, with scores ranging from -5.9 to +12.79 . All LIBRA factors could be operationalized in The Maastricht Study, except for the LIBRA factor high cognitive activity. Engagement in cognitively stimulating activities was not available in the dataset and, therefore, this LIBRA factor could not be included in the risk calculation. Available protective factors were adherence to a Mediterranean diet and low to moderate alcohol use. Risk factors were physical inactivity, smoking, obesity, depression, T2DM, hypertension, hypercholesterolemia, heart disease and chronic kidney disease. See Table 1 for an overview of all individual LIBRA factors, assigned weights and operationalization in this dataset.

Adherence to a Mediterranean diet was based on the Greek Mediterranean diet score derived from a comprehensive 253-item self-administered Food Frequency Questionnaire (FFQ) on frequency (not used to 7 days/week) and consumed amounts $\left(<1 /\right.$ day to $>12 /$ day), with a 1 -year reference period ${ }^{19}$. The Mediterranean diet score consists of the reported intake of vegetables, fruit and nuts, fish, cereal intake, dairy, meat and alcohol, with scores ranging from 0 to 9 . A score of $\geq 6$ is used as a cut-off for adhering to the diet ${ }^{20}$. Nonadherence to this diet does not necessarily imply non-adherence to the Dutch food-based dietary guidelines, which provide a more general guideline for a healthy diet in relation to numerous chronic diseases than specifically for brain health and dementia ${ }^{21}$. Physical inactivity was based on self-reported moderate to vigorous physical activity in the past two weeks, calculated from a modified version of the Community Healthy Activities Model Program for Seniors (CHAMPS) questionnaire ${ }^{22}$. Less than 150 minutes per week of moderate to vigorous physical activity was categorized as physically inactive, based on the Dutch physical activity guidelines ${ }^{23}$. Smoking status was defined by selfreported data on smoking cigarettes, with response options 'never smoked', 'ever smoked' and 'currently smoking': current smokers were assigned to the risk group. Low to moderate alcohol use was based on self-reported alcohol use per day based on an item of the FFQ, converted into grams of ethanol per day. Low to moderate alcohol intake was defined as $\leq 70$ grams per week, based on the Dutch guidelines recommending not to drink, or to drink no more than one glass of alcohol a day ${ }^{21}$. Obesity was based on the WHO categories ${ }^{24}$, in which a BMI (calculated from physical examination at the research centre) of $\geq 30$ $\mathrm{kg} / \mathrm{m}^{2}$ was defined as obese. The presence of depression was assessed using the Mini International Neuropsychiatric Interview (MINI; current major or minor depressive episode ${ }^{25}$. In case of missing data on the MINI, the Patient Health 
Questionnaire (PHQ-9) was used to determine presence of moderate to severe depressive symptoms (range 0-27; cut-off $\geq 10$ ) ${ }^{26}$. T2DM was defined based on glucose tolerance status based on fasting glucose $(\geq 7.0)$, oral glucose tolerance test ( $\geq 11.1$ ), according to WHO definition, or information on current diabetes medication use ${ }^{27}$. For sensitivity analyses, a second variable was computed based on impaired glucose metabolism, which includes both prediabetes and T2DM. Hypertension was based on average office blood pressure measurement (systolic blood pressure $\geq 140 \mathrm{mmHg}$ or diastolic blood pressure $\geq 90 \mathrm{mmHg}$ ) and/or current antihypertensive medication use. Hypercholesterolemia was calculated from serum total cholesterol using a cut-off of $\geq 6.5 \mathrm{mmol} / \mathrm{l}$. The LIBRA factor heart disease was based on self-reported history of cardiovascular disease from the Rose Questionnaire ${ }^{28}$ (i.e., myocardial infarction, and/or percutaneous artery angioplasty of the coronary arteries, abdominal arteries peripheral arteries or carotid artery, and/or vascular surgery on coronary arteries, abdominal arteries peripheral arteries or carotid artery). Presence of cerebrovascular infarction and haemorrhage were not included in the risk calculation of the LIBRA factor heart disease. For sensitivity analyses, a second variable was computed based only on self-reported history of myocardial infarction ${ }^{29}$, thereby including only coronary heart disease. Chronic kidney disease was derived from CKD-EPI equation estimated glomerular filtration rate using serum cystatin $C$ (serum cystatin $C$ of $<60$ ) and/or average urinary albumin excretion (both microalbuminuria and macro-albuminuria defined as risk $)^{30}$. 


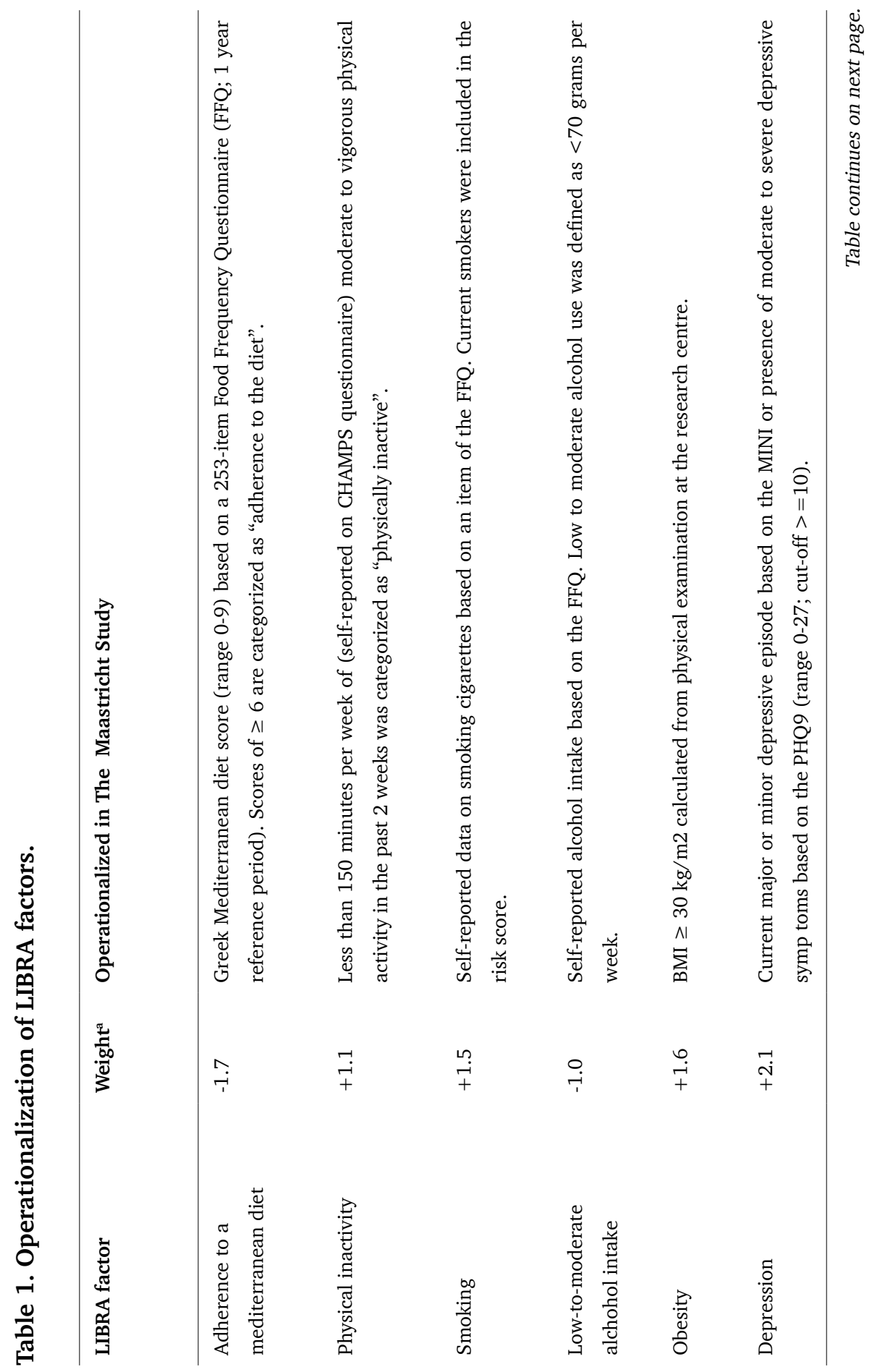




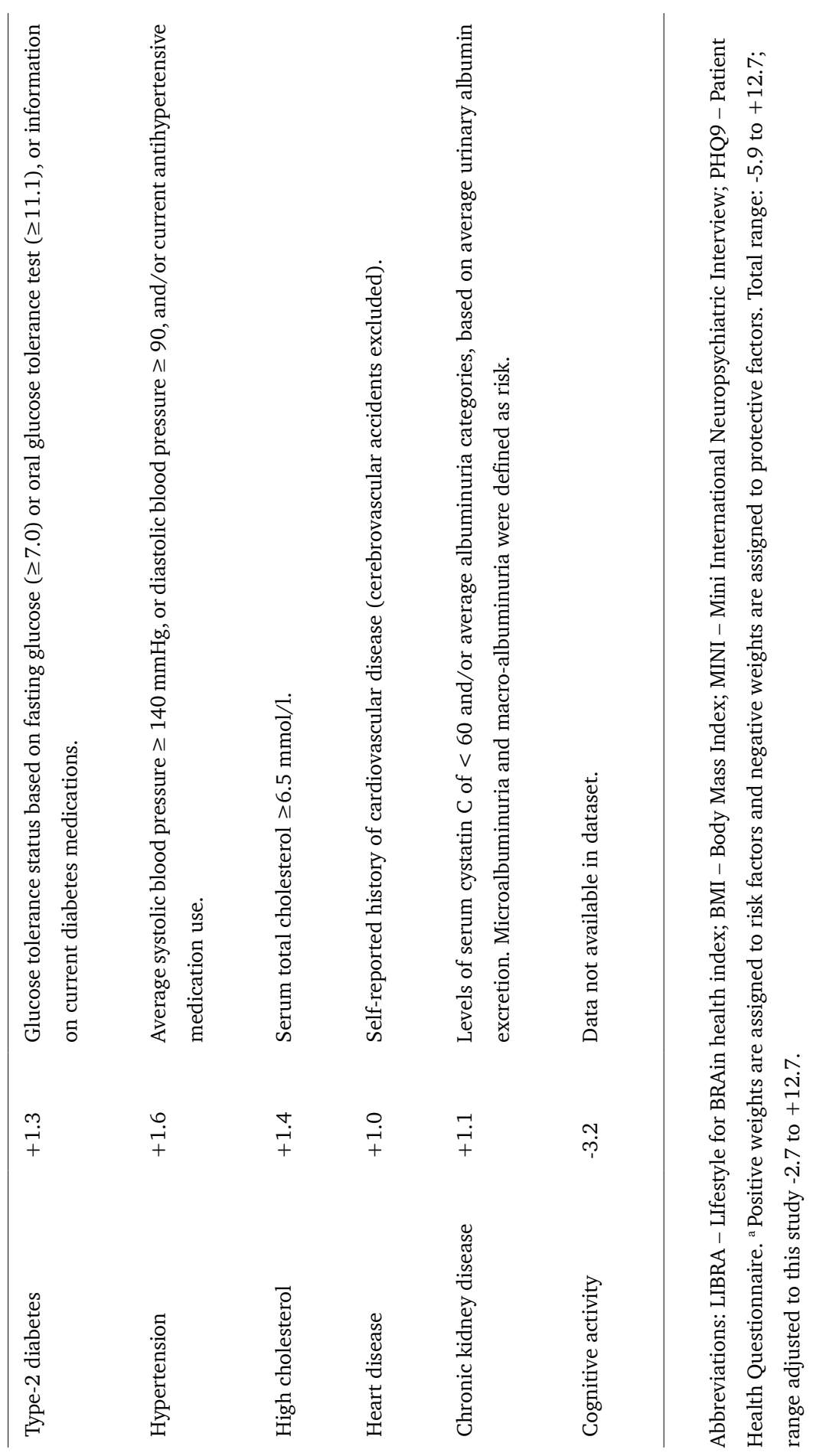




\section{Cognitive performance}

Cognitive performance was assessed by a concise (30-minute) neuropsychological test battery ${ }^{16}$. For conceptual clarity, individual neuropsychological test scores were standardized and divided into three cognitive domains (memory function, information processing speed, and executive function and attention (reprinted with permission) ${ }^{16,17}$. Briefly, memory function was evaluated using the Verbal Leaning Test ${ }^{31}$ and a memory domain score was derived by calculating the average of total immediate and delayed recall standardized scores. An information processing speed domain score was derived from standardized scores of the Stroop Colour-Word Test (SCWT) part I and $\mathrm{II}^{32}$, the Concept Shifting Test (CST) part A and $\mathrm{B}^{33}$ and the Letter-Digit Substitution $\mathrm{Test}^{34}$. The executive function and attention domain score was calculated from the average score of the SCWT part III and the CST part C. If necessary, individual test scores were log-transformed to reduce skewness of distributions and/or inverted so that higher scores indicated better cognitive performance. In addition, participants were categorized as cognitively impaired (yes/no) based on a regression-based normalization procedure per test that predicted expected scores for each individual given their age, sex and level of education from a published normative sample ${ }^{31-34}$. The difference between observed and expected scores and their standard deviation were used to calculate z-scores, which were then averaged per domain and re-standardized. Individuals performing $\leq 1.5 \mathrm{SD}$ below their norm-based expected score in any of the three cognitive domains were categorized as having cognitive impairment.

\section{Brain MRI}

Brain MRI was performed on a 3 Tesla (3T) MRI scanner (MAGNETOM Prismafit Syngo MR D13D; Siemens Healthcare, Erlangen, Germany) by use of a 64-element head coil for parallel imaging, as previously described ${ }^{16}$.

Measurement of brain volumes and cerebral small vessel disease T1 images and T2-weighted FLAIR images were analysed by use of an ISO13485:2012 certified automated method (which included visual inspection) ${ }^{35,36}$. T1 images were segmented into grey matter (GM), white matter (WM) and (as an inverted measure of brain atrophy) CSF ( 1 voxel $=1.00 \mathrm{~mm}^{3}=0.001$ $\mathrm{ml})^{35}$. Intracranial volume (ICV) was calculated as the sum of GM, WM and CSF. T2-weighted FLAIR and T1 images were used to calculate white matter hyperintensity $(\mathrm{WMH})$ volume ${ }^{36}$. Identified $\mathrm{WMHs}$ were summed to assess total 
WMH burden in ml. Additionally, WMHs were visually rated with the Fazekas scale $^{37}$. Lacunar infarcts and cerebral microbleeds were counted manually by three neuro-radiologists in accordance with the Microbleed Anatomical Rating Scale (MARS) ${ }^{38,39}$. Presence of cerebral small vessel disease (CSVD) was defined as a Fazekas score of $\geq 2$, presence of lacunar infarcts, and/or presence of cerebral microbleeds.

\section{Statistical analysis}

Independent samples t-tests and $\chi^{2}$-tests were used to investigate differences in demographic variables and LIBRA scores between the actual study sample used in the present study and the excluded group, and between three LIBRA groups (low risk: $\leq 1$ SD below sample mean, middle risk: between -1 and +1 SD, and high risk: $\geq 1$ SD above sample mean). The association between LIBRA and the structural MRI markers and between LIBRA and the three cognitive domains were analysed in separate multiple linear regression analyses. A quadratic term of LIBRA was added to the linear function in the analyses of the cognitive domains information processing speed and executive function and attention as this improved model fit. For direct comparison of strength of associations, we report the standardized regression coefficient beta and 95\% CI. Logistic regression analyses were used to examine the association between LIBRA and CSVD, and between LIBRA and cognitive impairment, yielding odds ratios (OR) and 95\% CIs.

Structural equation modelling was used to study mediation of LIBRA on cognition by MRI markers by decomposing the total association into direct and indirect associations. Because the regression analysis suggested a curvilinear association between LIBRA and two cognitive domains, we used a technique that allows for estimating non-linear mediation effects, which is not taken into account in traditional linear or log-linear mediation models (see Figure $1)^{40}$. For this, we estimated the instantaneous indirect effect $\theta$, which tests the mediation effect at different levels of the independent predictor variable (LIBRA), showing how the mediation effects changes as the level of the independent variable changes. Following this approach, we estimated the instantaneous indirect effects $\theta$ at three levels of LIBRA: 1 SD below the LIBRA sample mean (LIBRA score $=-0.87$ ), at the LIBRA sample mean (LIBRA score $=1.19$ ) and 1 SD above the LIBRA sample mean (LIBRA score $=3.25$ ), following previous recommendations ${ }^{40}$. To estimate robust $95 \%$ CIs, we used bootstrapping with 10,000 repetitions. 
Associations with cognition were adjusted for age, sex and level of education. Associations with structural brain markers were additionally adjusted for ICV to correct for head size, and the variable MRI lag time to adjust for the time (in years) between inclusion and MRI scan. The oversampling of participants with T2DM by design urged us to adjust for diabetes status in all the analyses in order to make sure that the overexpression of LIBRA risk factors in T2DM, such as obesity, hypercholesterolemia, hypertension or depression, did not confound the observed associations between LIBRA, MRI markers and cognition. Interaction terms were included in additional analyses to investigate whether the associations between LIBRA and brain markers or cognitive performance were moderated by sex and T2DM status. Finally, we did a series of sensitivity analyses to test the robustness of findings after assigning those with prediabetes the risk weight for T2DM, and after assigning a risk weight only to those with coronary heart disease. Statistical analyses were done with Stata 13.1 (StataCorp, TX) and Mplus8 (Muthen \& Muthen) using two-sided hypothesis testing and an alpha-level of $<.05$.

\section{Standard Protocol Approvals, Registrations, and Patient Consents}

The Maastricht Study has been approved by the institutional medical ethical committee (NL31329.068.10) and the Ministry of Health, Welfare and Sports of the Netherlands (Permit 131088-105234-PG). All participants gave their written informed consent ${ }^{16}$.

\section{Data Availability Statement}

Data are unsuitable for public deposition due to ethical restrictions and privacy regulation of participant data. Data from The Maastricht Study are available to any interested researcher who meets the criteria for access to confidential data. Data requests may be submitted to The Maastricht Study Management Team (research.dms@mumc.nl). 


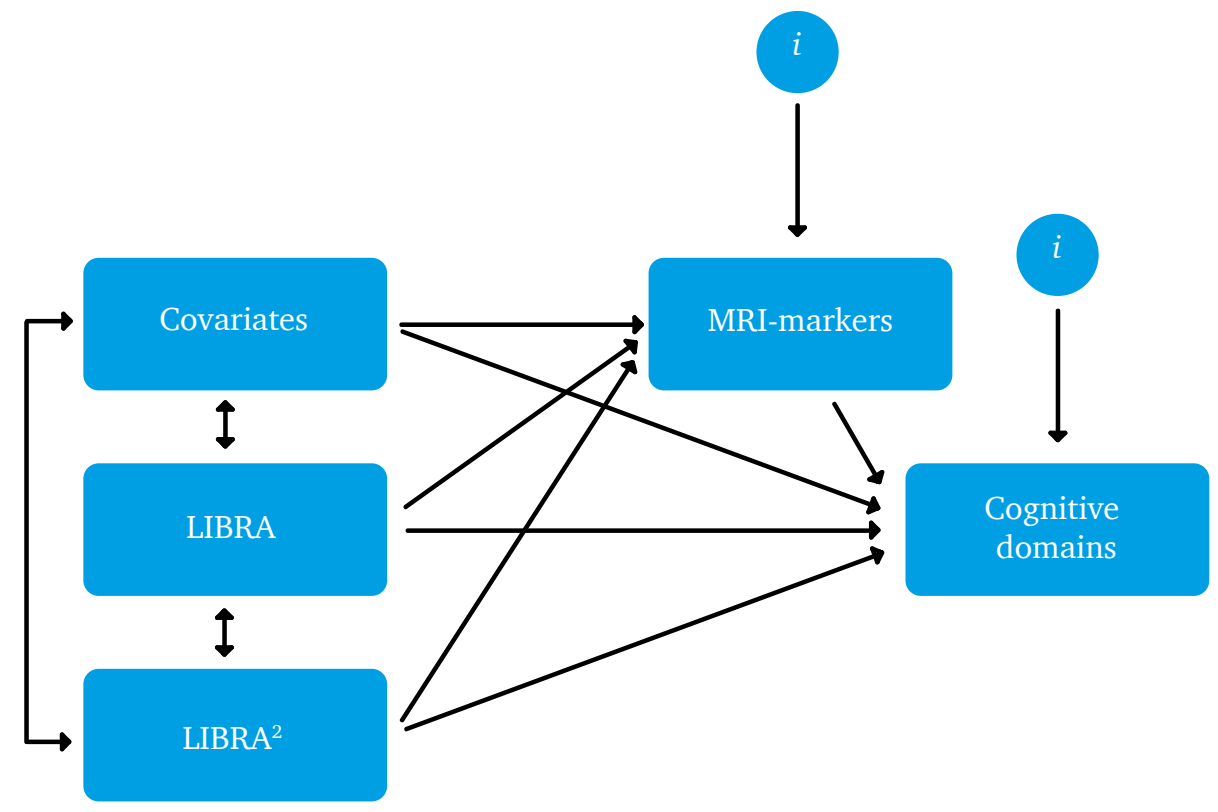

Figure 1. Path model to quantify the instantaneous indirect effect of LIBRA on cognition. Abbreviations: LIBRA - LIfestyle for BRAin health (continuous); LIBRA ${ }^{2}$ - LIfestyle for BRAin health (squared); ICV - intracranial volume. Covariates: sex, age, level of education, time between assessment and MRI, ICV and diabetes status. ${ }^{i}$ Standard error. 


\section{Results}

Study design and sample characteristics

Of all 7,689 participants (mean age 59.8 years; $50.4 \%$ men; $34.7 \%$ low educated; $24.6 \%$ T2DM), $45.8 \%$ was excluded from the present study, largely due to absence of MRI data. LIBRA factors that were most often missing were physical inactivity (9.8\% missing) and adherence to a Mediterranean diet and low to moderate alcohol intake (from the same food questionnaire; $5.2 \%$ missing). All other LIBRA factors were below 3.7\% missing. See Figure 2 for a flowchart. Compared to the study sample $(n=4,164)$, excluded participants $(\mathrm{n}=3,525)$ had a higher mean age $(59.2$ years vs. 60.5 years; $(\mathrm{t}(7687)=6.5, p$ $<0.001$ ) and had lower education (sample low education $30.2 \%$, excluded low education 40.2\%; $\left.\mathrm{X}^{2}(2)=86.6, p<0.001\right)$. Excluded participants had a more unfavourable LIBRA risk profile (1.19 vs. $1.95 ; \mathrm{t}(7687)=15.4, p<0.001)$, with higher presence of T2DM (19.0\% vs. 31.3\%; $\left.\mathrm{X}^{2}(1)=156.1, p<0.001\right)$, hypertension $\left(49.0 \%\right.$ vs. 59.7\%; $\left.\mathrm{X}^{2}(1)=87.0, p<0.001\right)$, heart disease (10.1\% vs. $20.3 \%$; $\left.X^{2}(1)=152.3, p<0.001\right)$, obesity $\left(18.0 \%\right.$ vs. $25.9 \%$; $X^{2}$ $(1)=70.9, p<0.001)$, chronic kidney disease (5.2\% vs. $7.6 \%$; $X^{2}(1)=19.3$, $p<0.001$ ), depression (4.2\% vs. 6.1\%; $\left.\mathrm{X}^{2}(1)=13.7, p<0.001\right)$, and were more often smokers $\left(11.0 \%\right.$ vs. $\left.16.4 \% ; X^{2}(1)=47.5, p<0.001\right)$, physically inactive $\left(25.3 \%\right.$ vs. $\left.31.6 \%, \mathrm{X}^{2}(1)=31.9, p<0.001\right)$ and less often adhered to the Mediterranean diet (28.5\% vs. $26.2 \%$; $\left.X^{2}(1)=4.6, p=0.032\right)$. Low to moderate alcohol intake was more common in the excluded group (54.9\% vs. 59.4\%; $\left.\mathrm{X}^{2}(1)=14.7, p<0.001\right)$ and hypercholesterolemia was more common in the study sample compared to excluded participants (15.4\% vs. $12.3 \%$; $\mathrm{X}^{2}$ $(1)=14.4, p<0.001)$. Men had higher (more unhealthy) average LIBRA scores (1.5) compared to women $(0.9 ; \mathrm{t}(4162)=10.3, p<0.001)$, including higher presence of T2DM (25.6\% vs. $\left.12.5 \%, \mathrm{X}^{2}(1)=116.1, p<0.001\right)$, hypertension $\left(57.7 \%\right.$ vs. $\left.40.4 \%, X^{2}(1)=125.1, p<0.001\right)$, and physical inactivity $(28.1 \%$ vs. $\left.22.5 \%, X^{2}(1)=17.1,<0.001\right)$. The characteristics of the total study sample and those with a low ( $\leq 1$ SD below sample mean), middle (between -1 and $+1 \mathrm{SD}$ ) and a high ( $\geq 1$ SD above sample mean) LIBRA score are summarized in Table 2. 
Total sample The Maastricht Study

$$
\mathbf{N}=7,689
$$

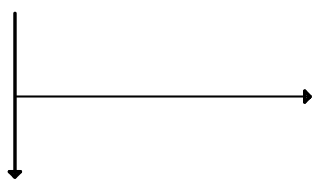

\section{Missing MRI data $(\mathrm{n}=2,506)$}

Sample with MRI data available $\mathrm{n}=5,183$

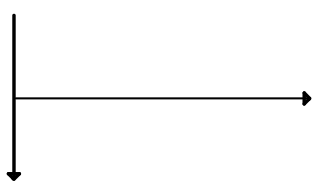

Sample with availability of 11 LIBRA factors $\mathrm{n}=4,300$

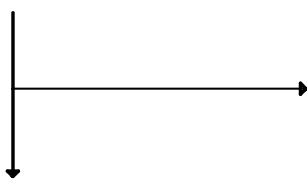

\section{Study sample} $\mathrm{n}=4,164$

Less than 11 LIBRA factors available $(n=883)$

Individual LIBRA factors missing:

Physical inactivity $(\mathrm{n}=507)$

Diet $(\mathrm{n}=270)$

Alcohol $(\mathrm{n}=269)$

Heart disease $(\mathrm{n}=193)$

Smoking $(\mathrm{n}=35)$

Hypertension $(n=5)$

Depression $(\mathrm{n}=5)$

Cholesterol $(n=4)$

Missing on all cognitive domains $(n=136)$

Individual domains missing:

Memory $(\mathrm{n}=153)$

Information processing speed $(\mathrm{n}=169)$

Executive functioning and attention $(\mathrm{n}=178)$

Figure 2. Flowchart of the study sample selection. Abbreviation: LIBRA - LIfestyle for BRAin health index. 
Table 2. Characteristics of the total sample and of participants with low, middle and high risk based on LIBRA scores.

\begin{tabular}{|c|c|c|c|c|}
\hline Variables $^{\mathrm{a}}$ & $\begin{array}{l}\text { Total sample } \\
\mathrm{N}=4,164\end{array}$ & $\begin{array}{l}\text { Low risk } k^{b} \\
\mathrm{n}=848\end{array}$ & $\begin{array}{l}\text { Middle risk } \\
\mathrm{n}=2,665\end{array}$ & $\begin{array}{l}\text { High risk }^{\mathrm{b}} \\
\mathrm{n}=651\end{array}$ \\
\hline Men; n (\%) & $2,070(49.7 \%)$ & 319 (37.6\%) & $1,354(50.8 \%)$ & $397(61.0 \%)$ \\
\hline Age; mean (SD) & $59.2(8.6)$ & $55.2(8.4)$ & $59.8(8.3)$ & $62.1(8.0)$ \\
\hline \multicolumn{5}{|l|}{ Education ${ }^{\mathrm{c}} ; \mathrm{n}(\%)$} \\
\hline Low & $1,252(30.2 \%)$ & $167(19.7 \%)$ & 795 (29.9\%) & $290(45.4 \%)$ \\
\hline Middle & $1,184(28.6 \%)$ & $255(30.1 \%)$ & $770(29.0 \%)$ & 159 (24.9\%) \\
\hline High & $1,706(41.2 \%)$ & $424(50.1 \%)$ & $1,092(41.1 \%)$ & $190(29.7 \%)$ \\
\hline \multicolumn{5}{|l|}{ Marital status; n (\%) } \\
\hline Single & $287(6.9 \%)$ & $64(7.6 \%)$ & $178(6.7 \%)$ & $45(6.9 \%)$ \\
\hline Married or registered & $3,417(82.1 \%)$ & $704(83.1 \%)$ & $2,199(82.5 \%)$ & $514(79.0 \%)$ \\
\hline Widowed/divorced & $452(10.9 \%)$ & $78(9.2 \%)$ & $283(10.6 \%)$ & $91(14.0 \%)$ \\
\hline Other & $7(0.2 \%)$ & $1(0.1 \%)$ & $5(0.2 \%)$ & $1(0.2 \%)$ \\
\hline
\end{tabular}

Table continues on next page. 


\begin{tabular}{|c|c|c|c|c|}
\hline $\begin{array}{l}\text { LIBRA total score; } \\
\text { mean (SD) }\end{array}$ & $1.19(2.06)$ & $-1.47(0.61)$ & $1.20(1.05)$ & $4.6(1.08)$ \\
\hline $\begin{array}{l}\text { Individual LIBRA } \\
\text { factors; n (\%) }\end{array}$ & & & & \\
\hline Type-2 diabetes & $790(19.0 \%)$ & $4(0.5 \%)$ & 390 (14.6\%) & $396(60.8 \%)$ \\
\hline Hypertension & $2,041(49.0 \%)$ & $61(7.2 \%)$ & $1,381(51.8 \%)$ & $599(92.0 \%)$ \\
\hline High cholesterol & $639(15.4 \%)$ & $27(3.2 \%)$ & $493(18.5 \%)$ & $119(18.3 \%)$ \\
\hline Mediterranean diet & $1,186(28.5 \%)$ & 457 (53.9\%) & $691(25.9 \%)$ & $38(5.8 \%)$ \\
\hline Heart disease & 419 (10.1) & $10(1.2 \%)$ & $254(9.5 \%)$ & $155(23.8 \%)$ \\
\hline $\begin{array}{l}\text { Chronic kidney } \\
\text { disease }\end{array}$ & $216(5.2 \%)$ & $3(0.4 \%)$ & $117(4.4 \%)$ & $96(14.8 \%)$ \\
\hline $\begin{array}{l}\text { Low/moderate } \\
\text { alcohol use }\end{array}$ & $2,285(54.9 \%)$ & $677(79.8 \%)$ & $1,258(47.2 \%)$ & $350(53.8 \%)$ \\
\hline Physical inactivity & $1,054(25.3 \%)$ & $23(2.7 \%)$ & 677 (25.4\%) & $354(54.4 \%)$ \\
\hline Depression & $175(4.2 \%)$ & $0(0 \%)$ & $67(2.5 \%)$ & $108(16.6 \%)$ \\
\hline Obesity & $749(18.0 \%)$ & $6(0.7 \%)$ & $320(12.0 \%)$ & $423(65.0 \%)$ \\
\hline Smoking & $458(11.0 \%)$ & $12(1.4 \%)$ & $300(11.3 \%)$ & $146(22.4 \%)$ \\
\hline
\end{tabular}

Abbreviations: LIBRA - LIfestyle for BRAin Health score (higher is more risk).

${ }^{\text {a }}$ Maximum values and percentages do not count up due to missing values and rounding issues; ${ }^{\mathrm{b}}$ Low (risk) score $=\leq 1 \mathrm{SD}$ below sample mean, middle (risk) score $=$ between -1 and $+1 \mathrm{SD}$, high (risk) score $=\geq 1 \mathrm{SD}$ above sample mean; ${ }^{\mathrm{c}}$ Educational level was divided from 9 ordinal levels to 3 categories (low: no education, primary education, lower vocational education; middle: intermediate vocational education, higher secondary education; high: higher professional education, university education). 


\section{LIBRA and structural brain measures}

Table 3 displays the results of the multiple linear regression analyses of the association between LIBRA and the volumetric MRI markers. Higher LIBRA scores were linearly associated with higher volumes of WMH in the total sample. Interaction analyses revealed that the association between LIBRA and GM and CSF volume were moderated by sex, with stronger and significant associations in men, but associations in women were directionally similar (Figure 3A and B). No association was found between LIBRA and volumes of WM, and no interactions were found by T2DM status. There was no association between the LIBRA score and presence of CSVD (OR 1.036, CI $0.994-1.080, p=0.092$ ). When applying a stricter definition of CSVD, defined as the presence of at least two markers of CSVD, an association was found (OR 1.123, CI 1.028-1.226, $p=0.010$ ). 


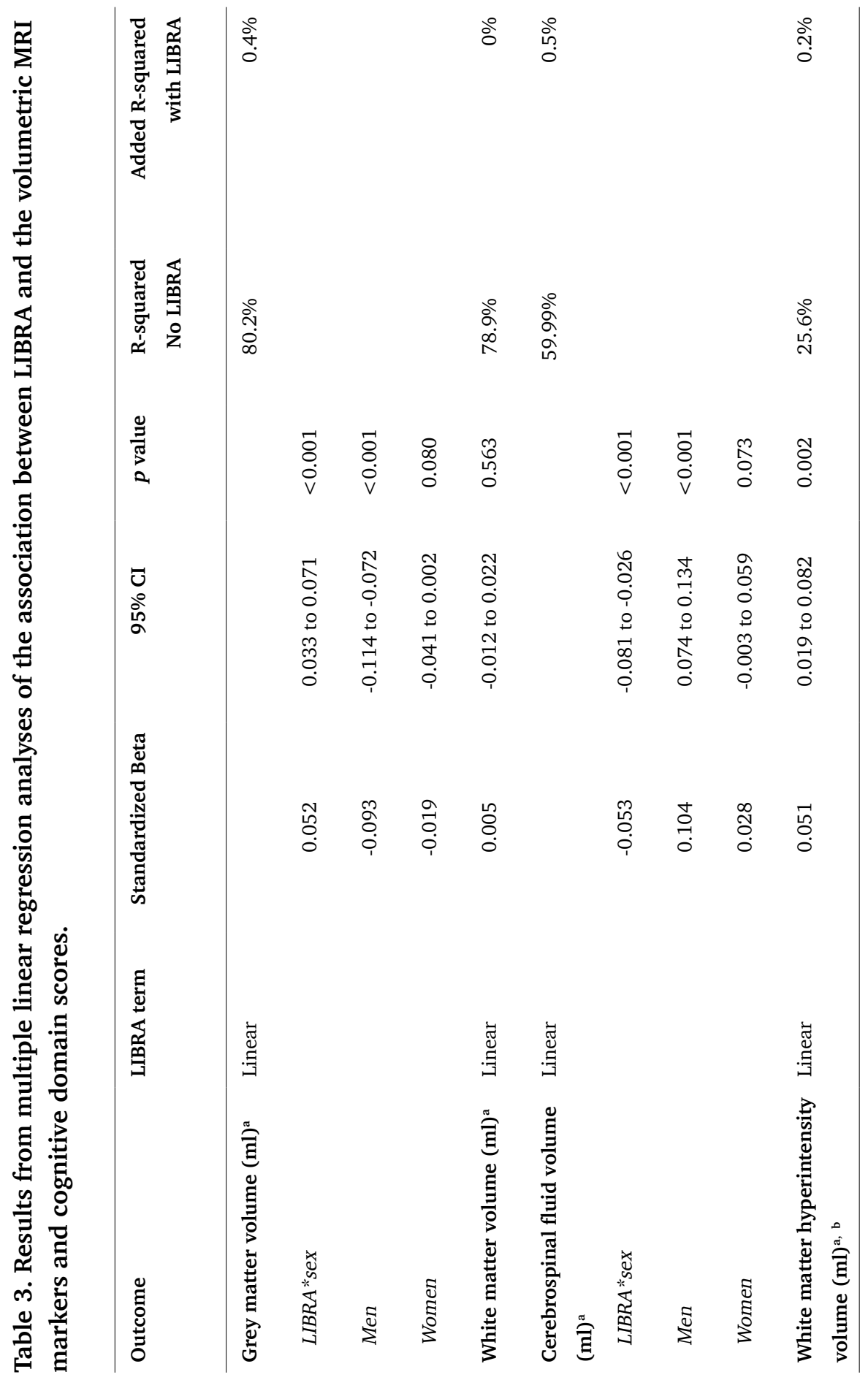




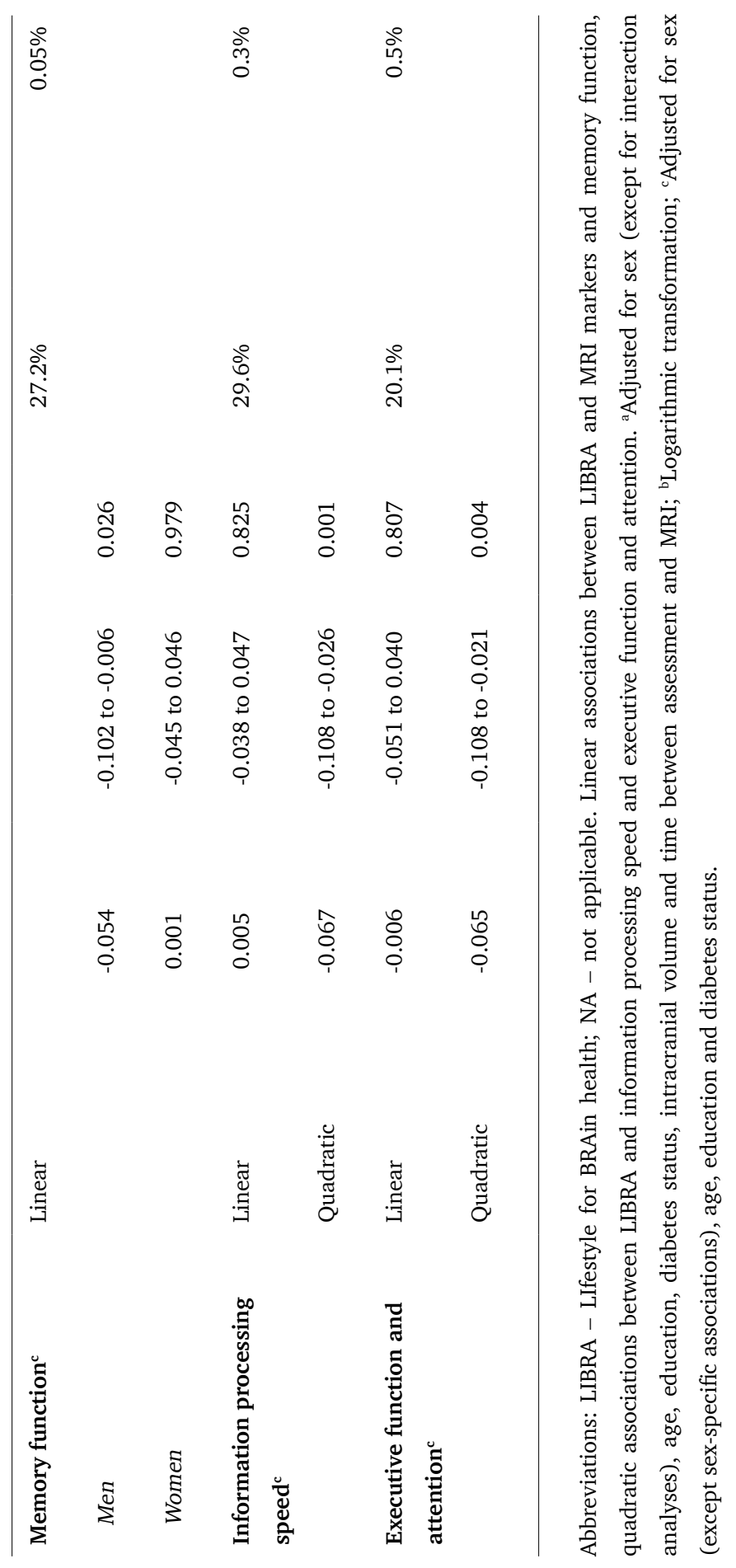



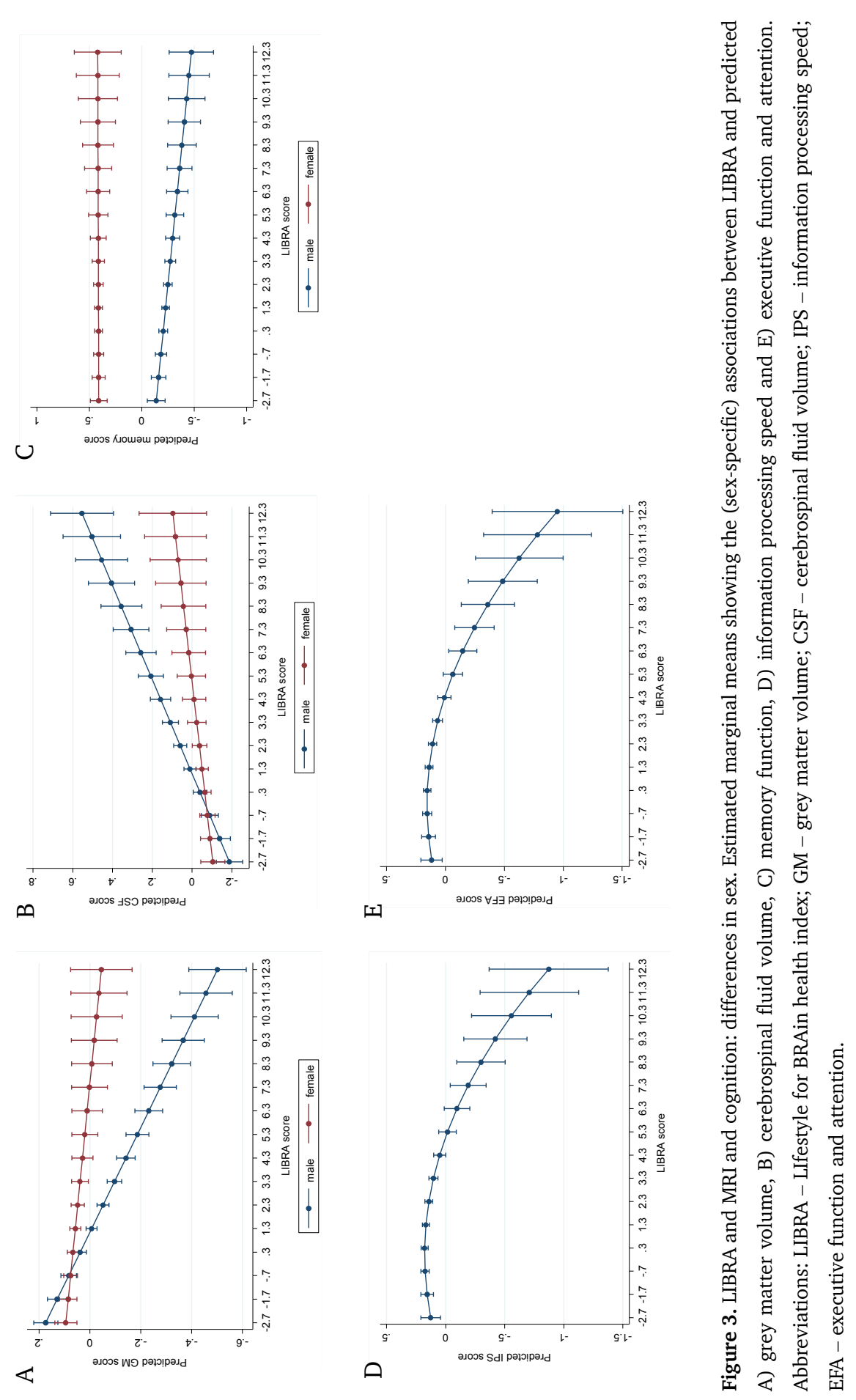


\section{LIBRA and cognition}

Likelihood ratio testing of the association between LIBRA and cognition showed that the model including both a linear and a quadratic LIBRA term had the best fit for the cognitive domains information processing speed and executive function and attention. As shown in Figure 3 (C-E), the relationship between LIBRA and these two domains changed as LIBRA scores increased in a curvilinear fashion, with a stronger negative association as LIBRA scores increased. A linear LIBRA term was the best fit for the domain of memory function. The results of the regression analyses are displayed in Table 3. Wald tests of the joint effects of the combined linear-quadratic LIBRA term were significant for both information processing speed: $(\mathrm{F}(2,4099)=$ 9.08, $p<0.001)$, and executive function and attention: $(\mathrm{F}(2,4090)=9.14$, $p<0.001)$. Additionally, we filtered the model only to cognition scores $>-1$ and then performed Wald tests to test if the quadratic LIBRA still improved the model. Wald tests were significant, both for information processing speed $(p<0.001)$ and executive function and attention $(p=0.007)$. No interactions were found for sex and T2DM status. Sex-specific analyses suggested that the effect for memory function was only present in men (see Figure 3C and Table 3).

\section{Cognitive impairment}

Likelihood ratio testing showed that the model including both a linear and a quadratic LIBRA term had the best fit for cognitive impairment. Logistic regression analyses revealed a relationship between the quadratic LIBRA score and the odds of cognitive impairment (OR 1.02, CI 1.006 - 1.036, $p=0.006$ ).

Mediation analyses of the association between LIBRA and cognitive outcomes by MRI markers

Non-linear mediation at different levels of LIBRA (low: -1 SD, middle: at mean, high: +1 SD) showed that WMH volumes partly explained the relationship between LIBRA and information processing speed, executive function and attention, and cognitive impairment in the total sample. Following the observed curvilinear association between LIBRA and these cognitive outcomes, the non-linear mediation effect $\theta$ tended to increase across levels of LIBRA. This suggests that MRI markers partly mediated the association between LIBRA and cognitive outcomes, and this became even stronger as LIBRA increased. Higher CSF volumes also mediated the association between LIBRA and information processing speed and between LIBRA and executive function and attention. 
In men only, WMH volumes mediated the association between LIBRA and memory function. The details on the estimations of the (instantaneous) indirect associations of LIBRA on cognition through MRI are given in Table 4. 


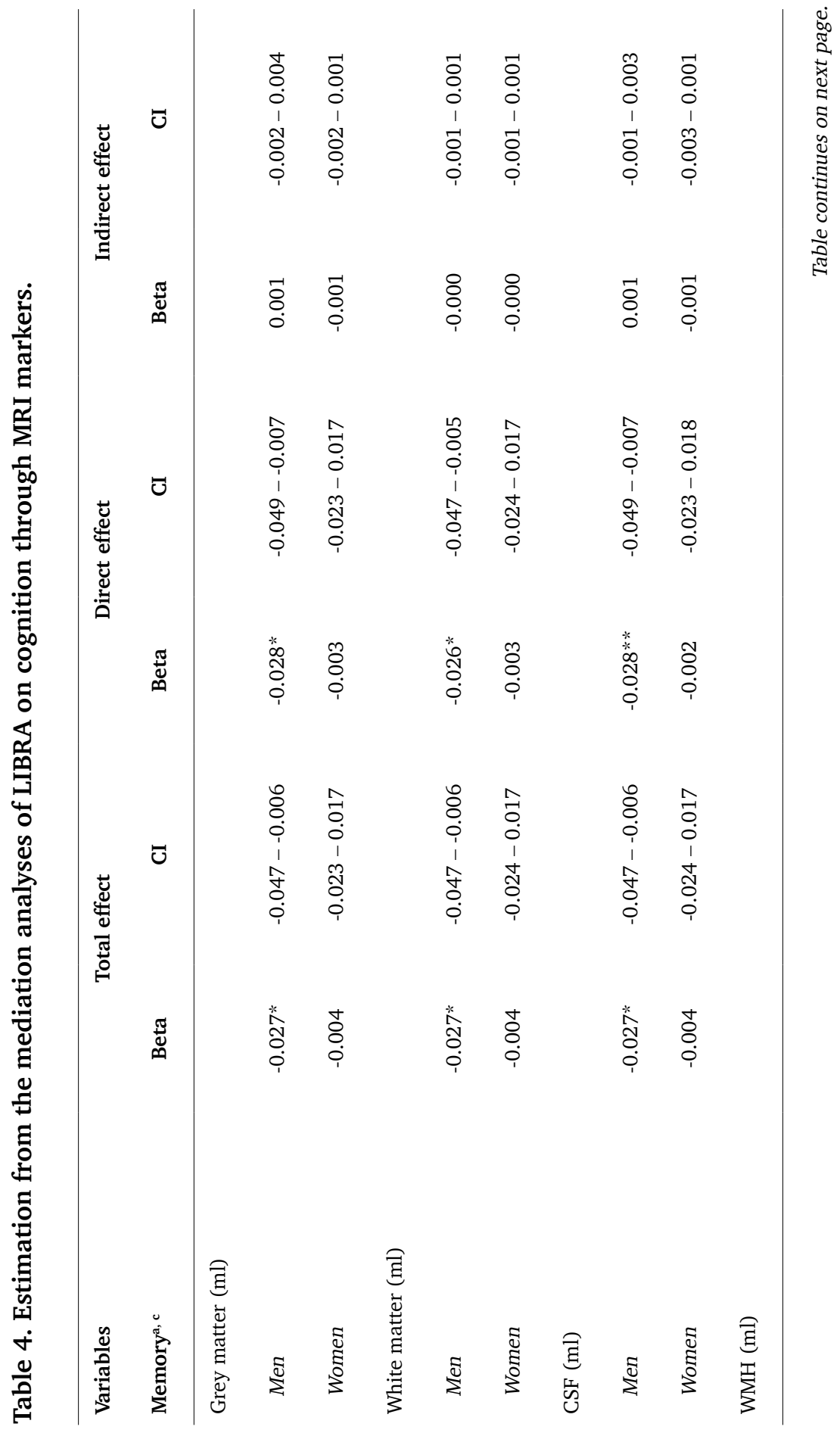




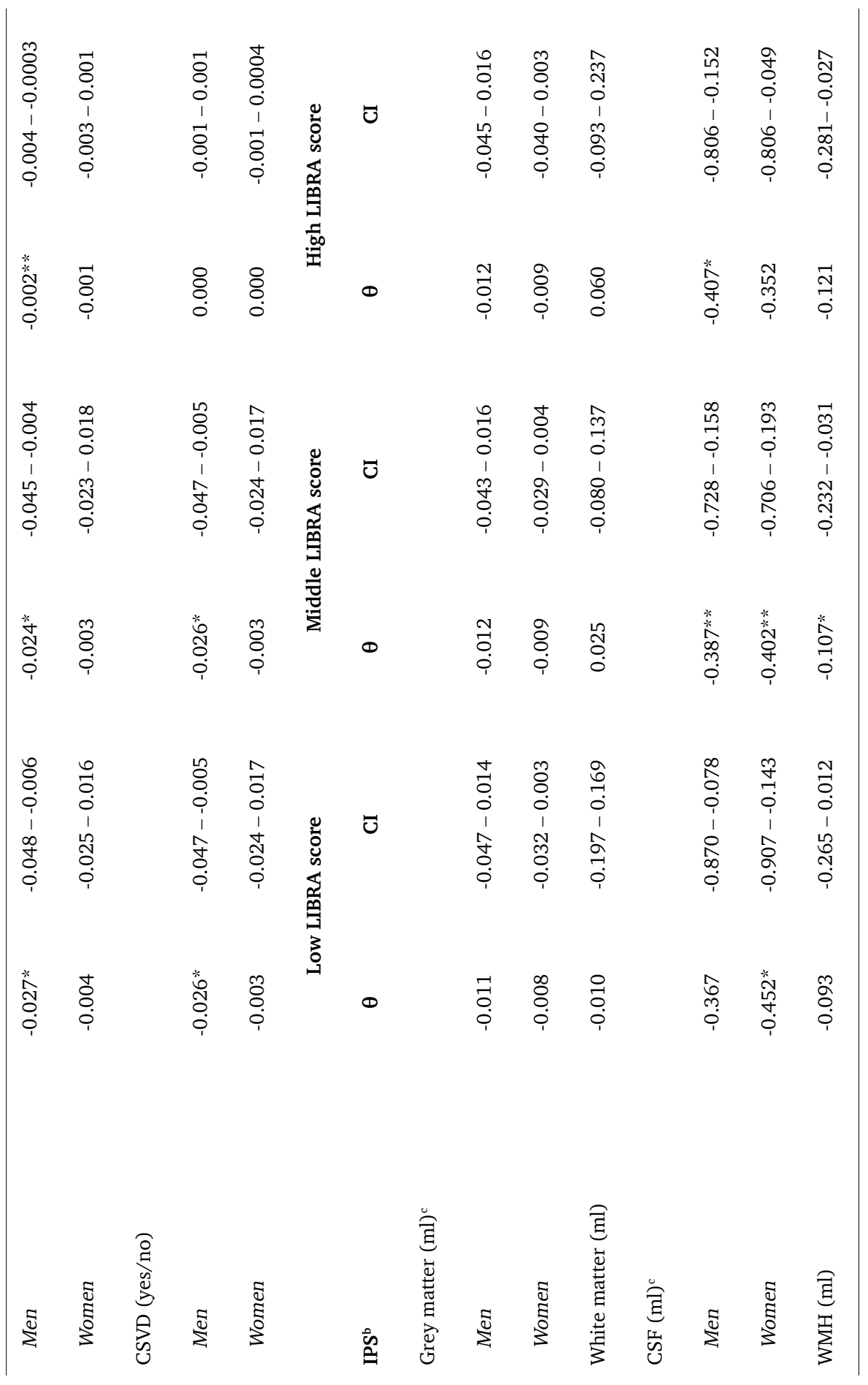




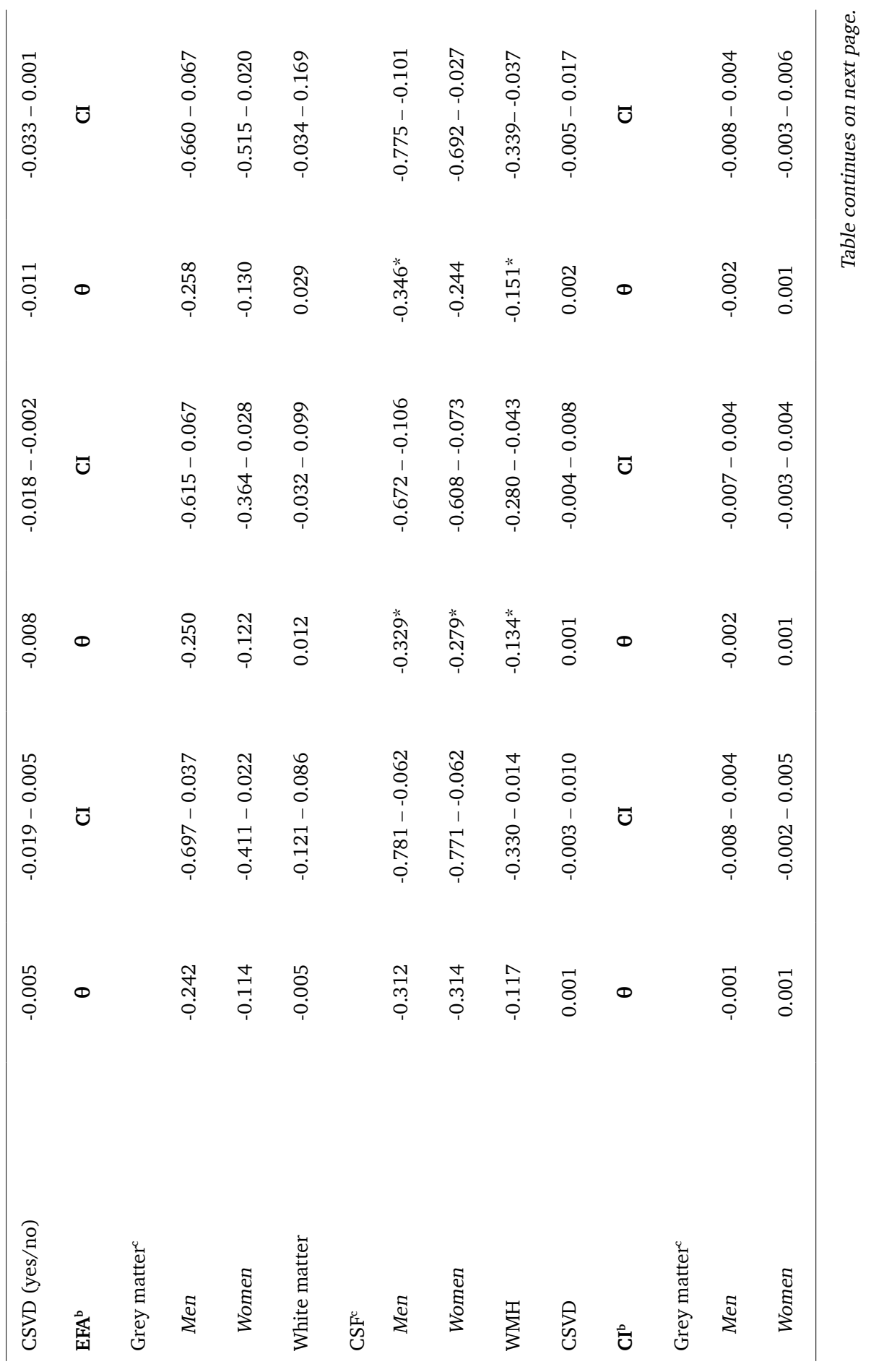




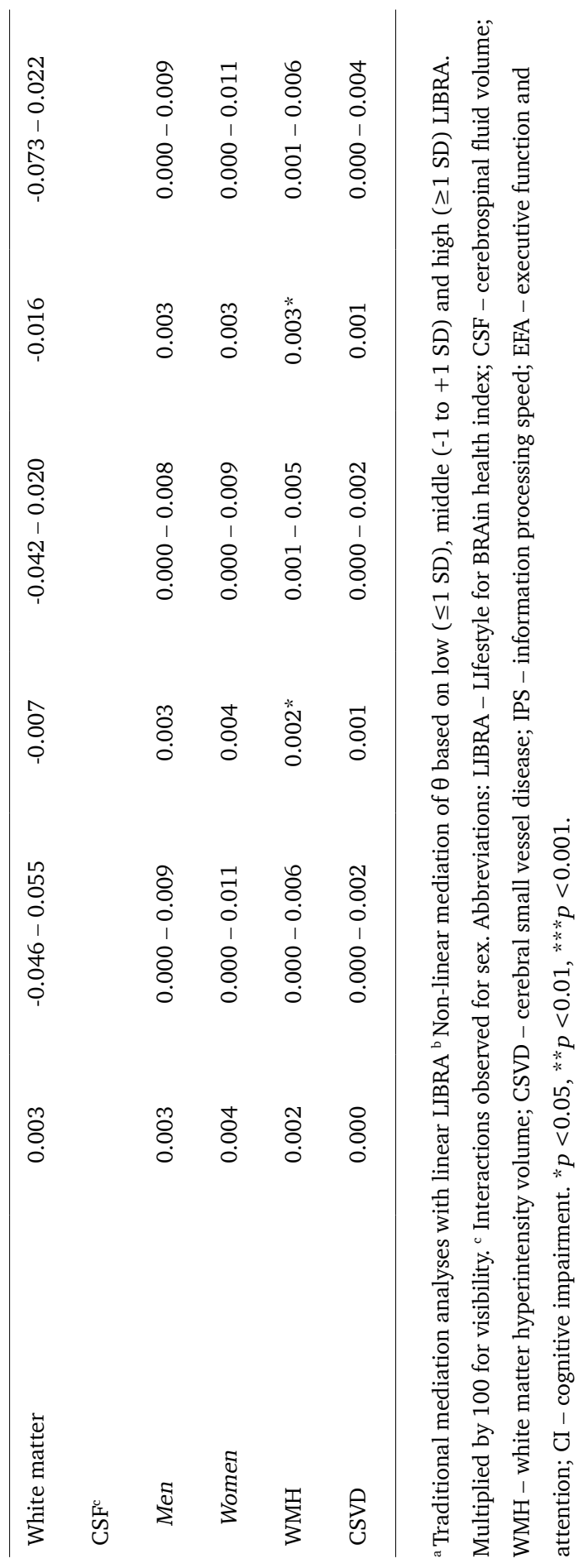




\section{Additional analyses}

Sensitivity analyses were performed for the LIBRA variables T2DM, assigning those with prediabetes the risk-weight for T2DM, and for heart disease, by assigning a risk-weight only to those with coronary heart disease (in line with its initial use in $\mathrm{LIBRA}^{29}$ ). Results remained similar to the main analyses. 


\section{Discussion}

This cross-sectional population-based study investigated the relationship of a modifiable risk score for dementia with brain-MRI markers and cognitive functioning. Higher LIBRA scores, reflecting a less brain-healthy lifestyle, were associated with $\mathrm{WMH}$ volume and with lower scores on information processing speed and executive function and attention and higher odds of cognitive impairment. Associations of LIBRA with memory and general brain atrophy (i.e., GM, CSF) were only present in men. Volumes of WMH and CSF mediated the association between LIBRA and cognition in the full cohort, and WMH mediated the relation with memory in men.

The results confirm previous studies showing that higher LIBRA scores are related to lower cognitive functioning and higher risk for cognitive impairment and dementia in the general population and clinical studies ${ }^{10-14}$. Importantly, our study shows a relationship of LIBRA with underlying biological gradients of $\mathrm{WMH}$ and global atrophy using population MRI imaging, showing it is indeed an index of brain health. In men, higher LIBRA scores were associated with higher volumes of brain atrophy and lower scores on memory function, with directionally similar but not significant associations in women. Although the association of LIBRA with memory in men was only found in sex-specific analyses, and not in formal interaction analyses as has been found for the association with brain atrophy, these two findings seem congruent. Both memory decline and brain atrophy are manifestations of Alzheimer's disease ${ }^{41}$, and previous studies showed that, in middle-age, men have more pronounced brain atrophy compared to women, whereas women show steeper decline in later phases ${ }^{42-44}$. Lifestyle-related brain damage might thus be more pronounced in men compared to women of the same age in our cohort aged 40-75 years, leading to lower cognitive performance. Indeed, men had higher mean LIBRA scores, which is in line with a previous study ${ }^{13}$, and higher WMH and CSF volume and worse cognitive scores, including memory, than women in the present study. The fact that worse cognitive performance was more strongly related with MRI markers as LIBRA scores increased, adds to the validity of this score for identifying those with low brain health and high risk of deterioration.

Various pathophysiological mechanisms may affect the different LIBRA factors, such as arteriolosclerosis ${ }^{45}$, atherosclerotic burden ${ }^{46}$, cerebral hypoperfusion ${ }^{46}$, and neurodegenerative Alzheimer's disease pathology ${ }^{47}$. We only found an association between LIBRA and presence of CSVD when holding a more strict 
definition of CSVD, which was not the initial a priori definition. Interestingly, both WMH and brain atrophy explained the relation between LIBRA and cognition. While cross-sectional associations do not allow temporal inference, it is in line with the idea that these risk factors affect and accelerate both vascular and neurodegenerative pathology ${ }^{48}$. In line with our study, the Cardiovascular Risk Factors, Aging, and Incidence of Dementia (CAIDE) Risk Score has been associated with WMH load, too ${ }^{49}$. This score includes both modifiable (hypertension, hypercholesterolemia, BMI and physical inactivity) and non-modifiable factors (e.g., age, sex, education), which makes it difficult to disentangle their relative contribution. Besides, an external validation study of four dementia prediction models (including CAIDE) showed that age alone already showed nearly identical discriminative ability compared to the full model including other (modifiable) risk factor ${ }^{50}$. Importantly, we showed that a compound score of health- and lifestyle factors is associated with brain markers and cognition, even after adjustment for the contribution of the non-modifiable factors age, sex and education. The use of a compound score that includes risk and protective factors all within the reach of vascular risk management and lifestyle interventions makes the LIBRA index a useful tool in identifying a group of individuals at increased risk of cognitive decline and dementia and for monitoring treatment targets over time in dementia prevention trials ${ }^{15}$.

Strengths of this study include the population-based design, the large sample size within the midlife target age range (40-75 years), which makes the results particularly generalizable to middle-aged individuals. Furthermore, the availability of domain-specific cognition and state-of-the-art populationimaging data allowed us to study the association pathways in considerable depth. The extensive phenotyping approach of The Maastricht Study made it possible to operationalize the LIBRA factors largely based on objective clinical data from physical examination at the research centre, if applicable in combination with self-reported data. Last, this study has taken the nonlinear association between LIBRA and cognition into account in the mediation analysis by estimating the instantaneous indirect effects of the MRI markers. Unfortunately, these analyses made it impossible to quantify the overall indirect effect of LIBRA on cognition through the MRI markers since the instantaneous indirect effect is estimated for three specific values of LIBRA (based on the $\mathrm{SD})^{40}$. Other limitations of our study are the cross-sectional design, in which definitive conclusions concerning cause and effect are not possible. Also, 
selection bias may have occurred in this present study due to missing MRI data. Indeed, the group that did not undergo an MRI, and was therefore not included in this study, was older, had a lower level of education and appeared to be more frail, i.e., higher presence LIBRA factors such as T2DM, hypertension and heart disease, which likely have led to an underestimation of the associations. Next, while data on most LIBRA factors were available in this dataset, the absence of the LIBRA factor cognitive activity, which is the strongest protective factor (LIBRA weight of -3.2), could have weakened the predictive value of the LIBRA index. Furthermore, the use of dichotomous LIBRA scores, i.e., presence of LIBRA factor yes/no, makes the index less suitable to detect small changes in a specific factor in behavioral change programs ${ }^{15}$. Yet, a study showed that LIBRA was most responsive to change compared to other risk indices, probably due to the large amount of modifiable factors ${ }^{15}$. Still, the use of alternative scoring formats need to be considered. Finally, the adjustment for T2DM status in all analyses might not be sufficient to control for the oversampling of participants with T2DM by design. There was, however, no interaction pattern for T2DM, suggesting that LIBRA had similar associations on cognition in those with and without T2DM.

Future studies should replicate these findings in a prospective design, in order to expand the understanding of the relationship between health- and lifestylerelated risk factors and cognitive ageing over time. Furthermore, the mediation analyses should be explored further by more extensive brain structure measures (e.g., white matter connectivity, hippocampal volume).

\section{Conclusion}

This study showed that higher LIBRA scores, indicating a less brain-healthy lifestyle profile, are associated with lower information processing speed, executive function and attention and white matter hyperintensities in the total population, and with lower memory function and markers of global brain atrophy in men, independent of the non-modifiable risk factors age, sex and education. Sex differences in the lifestyle-related pathology and manifestations of dementia need to be further explored. Improving health and lifestyle factors captured by LIBRA might improve population brain health. 


\section{References}

1. Norton S, Matthews FE, Barnes DE, Yaffe K, Brayne C. Potential for primary prevention of Alzheimer's disease: an analysis of population-based data. Lancet Neurol 2014;13:788-794.

2. Livingston G, Huntley J, Sommerlad A, et al. Dementia prevention, intervention, and care: 2020 report of the Lancet Commission. Lancet 2020;396:413-446.

3. Risk reduction of cognitive decline and dementia: WHO guidelines. 2019, World Health Organization: Geneva.

4. Stephen R, Liu Y, Ngandu T, et al. Associations of CAIDE Dementia Risk Score with MRI, PIB-PET measures, and cognition. $J$ Alzheimers Dis 2017;59:695-705.

5. Kivipelto M, Ngandu T, Laatikainen T, Winblad B, Soininen H, Tuomilehto J. Risk score for the prediction of dementia risk in 20 years among middle aged people: a longitudinal, population-based study. Lancet Neurol 2006;5:735-741.

6. Reitz C, Tang MX, Schupf N, Manly JJ, Mayeux R, Luchsinger JA. A summary risk score for the prediction of Alzheimer disease in elderly persons. Arch Neurol 2010;67:835-841.

7. Cherbuin N, Shaw ME, Walsh E, Sachdev P, Anstey KJ. Validated Alzheimer's Disease Risk Index (ANUADRI) is associated with smaller volumes in the default mode network in the early 60s. Brain Imaging Behav
2019;13:65-74.

8. Barnes DE, Yaffe K. Predicting dementia: role of dementia risk indices. Future Neurol 2009;4:555-560.

9. Deckers K, van Boxtel MP, Schiepers OJ, et al. Target risk factors for dementia prevention: a systematic review and Delphi consensus study on the evidence from observational studies. Int $J$ Geriatr Psychiatry 2015;30:234-246.

10. Schiepers OJG, Kohler S, Deckers K, et al. Lifestyle for Brain Health (LIBRA): a new model for dementia prevention. Int $J$ Geriatr Psychiatry 2018;33:167-175.

11. Vos SJB, van Boxtel MPJ, Schiepers OJG, et al. Modifiable Risk Factors for Prevention of Dementia in Midlife, Late Life and the Oldest-Old: Validation of the LIBRA Index. $J$ Alzheimers Dis 2017;58:537-547.

12. Pons A, LaMonica HM, Mowszowski L, Kohler S, Deckers K, Naismith SL. Utility of the LIBRA Index in Relation to Cognitive Functioning in a Clinical Health Seeking Sample. $J$ Alzheimers Dis 2018;62:373-384.

13. Deckers K, Nooyens A, van Boxtel M, Verhey F, Verschuren M, Kohler S. Gender and Educational Differences in the Association between Lifestyle and Cognitive Decline over 10 Years: The Doetinchem Cohort Study. J Alzheimers Dis 2018;70:S31-S41.

14. Deckers $K$, Barbera $M$, Köhler $S$, et al. Long-term dementia risk prediction by the LIBRA score: A 30-year follow-up of 
the CAIDE study. Int $J$ Geriatr Psychiatry 2020;35:195-203.

15. Coley N, Hoevenaar-Blom MP, van Dalen JW, et al. Dementia risk scores as surrogate outcomes for lifestyle-based multidomain prevention trials-rationale, preliminary evidence and challenges. Alzheimers Dement 2020.

16. Schram MT, Sep SJS, van der Kallen CJ, et al. The Maastricht Study: an extensive phenotyping study on determinants of type 2 diabetes, its complications and its comorbidities. Eur $J$ Epidemiol 2014;29:439-451.

17. Geraets AFJ, Schram MT, Jansen JFA, et al. The relation of depression with structural brain abnormalities and cognitive functioning: the Maastricht study. Psychol Med 2021:1-10.

18. Deckers K, Kohler S, van Boxtel M, Verhey F, Brayne C, Fleming J. Lack of associations between modifiable risk factors and dementia in the very old: findings from the Cambridge City over-75s cohort study. Aging Ment Health 2017;22:1272-1278.

19. van Dongen MC, Wijckmans-Duysens NEG, den Biggelaar LJ, et al. The Maastricht FFQ: Development and validation of a comprehensive food frequency questionnaire for the Maastricht study. Nutrition 2019;62:39-46.

20. Trichopoulou A, Orfanos P, Norat T, et al. Modified Mediterranean diet and survival: EPIC-elderly prospective cohort study. BMJ 2005;330:991.

21. Kromhout D, Spaaij CJK, de Goede J, Weggemans RM. The 2015 Dutch foodbased dietary guidelines. Eur J Clin Nutr
2016;70:869.

22. Resnicow $\mathrm{K}$, McCarty $\mathrm{F}$, Blissett $\mathrm{D}$, Wang $\mathrm{T}$, Heitzler C, Lee RE. Validity of a modified CHAMPS physical activity questionnaire among African-Americans. Med Sci Sports Exerc 2003;35:1537-1545.

23. Weggemans RM, Backx FJG, Borghouts L, et al. The 2017 Dutch Physical Activity Guidelines. Int $J$ Behav Nutr Phys Act 2018;15:58.

24. World Health Organization. Obesity. 2019; Beschikbaar op: https://www.who. int/topics/obesity/en/. Geraadpleegd op 27-2-2019.

25. Sheehan DV, Lecrubier Y, Sheehan KH, et al. The Mini-International Neuropsychiatric Interview (M.I.N.I.): the development and validation of a structured diagnostic psychiatric interview for DSM-IV and ICD-10. J Clin Psychiatry 1998;59 Suppl 20:22-57

26. Kroenke K, Spitzer RL, Williams JB. The PHQ-9: validity of a brief depression severity measure. $J$ Gen Intern Med 2001;16:606-613.

27. Definition and diagnosis of diabetes mellitus and intermediate hyperglycaemia: report of a WHO/ IDF consultation. 2006, World Health Organization: Geneva, Switzerland.

28. Rose G, McCartney P, Reid DD. Selfadministration of a questionnaire on chest pain and intermittent claudication. Br J Prev Soc Med 1977;31:42-48.

29. Deckers K, Schievink SHJ, Rodriquez MMF, et al. Coronary heart disease and risk for cognitive impairment or dementia: Systematic review and meta- 
analysis. PLoS One 2017;12.

30. Inker LA, Schmid CH, Tighiouart $\mathrm{H}$, et al. Estimating glomerular filtration rate from serum creatinine and cystatin C. $N$ Engl $J$ Med 2012;367:20-29.

31. Van der Elst W, van Boxtel MP, van Breukelen GJ, Jolles J. Rey's verbal learning test: normative data for 1855 healthy participants aged 24-81 years and the influence of age, sex, education, and mode of presentation. $J$ Int Neuropsychol Soc 2005;11:290-302.

32. Van der Elst W, Van Boxtel MP, Van Breukelen GJ, Jolles J. The Stroop colorword test: influence of age, sex, and education; and normative data for a large sample across the adult age range. Assessment 2006;13:62-79.

33. Van der Elst W, Van Boxtel MP, Van Breukelen GJ, Jolles J. The Concept Shifting Test: adult normative data. Psychol Assess 2006;18:424-432.

34. van der Elst W, van Boxtel MP, van Breukelen GJ, Jolles J. The Letter Digit Substitution Test: normative data for 1,858 healthy participants aged 24-81 from the Maastricht Aging Study (MAAS): influence of age, education, and sex. $J$ Clin Exp Neuropsychol 2006;28:9981009.

35. Vrooman HA, Cocosco CA, van der Lijn F, et al. Multi-spectral brain tissue segmentation using automatically trained k-Nearest-Neighbor classification. Neuroimage 2007;37:71-81.

36. de Boer R, Vrooman HA, van der Lijn F, et al. White matter lesion extension to automatic brain tissue segmentation on
MRI. Neuroimage 2009;45:1151-1161.

37. Fazekas F, Chawluk JB, Alavi A, Hurtig HI, Zimmerman RA. MR signal abnormalities at $1.5 \mathrm{~T}$ in Alzheimer's dementia and normal aging. AJR Am $J$ Roentgenol 1987;149:351-356.

38. Cordonnier C, Potter GM, Jackson CA, et al. improving interrater agreement about brain microbleeds: development of the Brain Observer MicroBleed Scale (BOMBS). Stroke 2009;40:94-99.

39. Gregoire SM, Chaudhary UJ, Brown MM, et al. The Microbleed Anatomical Rating Scale (MARS): reliability of a tool to map brain microbleeds. Neurology 2009;73:1759-1766.

40. Hayes AF, Preacher KJ. Quantifying and Testing Indirect Effects in Simple Mediation Models When the Constituent Paths Are Nonlinear. Multivariate Behav Res 2010;45:627-660.

41. Lane CA, Hardy J, Schott JM. Alzheimer's disease. Eur J Neurol 2018;25:59-70.

42. Ferretti MT, Iulita MF, Cavedo E, et al. Sex differences in Alzheimer disease - the gateway to precision medicine. Nat Rev Neurol 2018;14:457-469.

43. Ferretti MT, Martinkova J, Biskup E, et al. Sex and gender differences in Alzheimer's disease: current challenges and implications for clinical practice: Position paper of the Dementia and Cognitive Disorders Panel of the European Academy of Neurology. Eur J Neurol 2020;27:928943.

44. Armstrong NM, An Y, Beason-Held L, et al. Sex differences in brain aging and predictors of neurodegeneration 
in cognitively healthy older adults. Neurobiol Aging 2019;81:146-156.

45. Berry C, Sidik N, Pereira AC, et al. SmallVessel Disease in the Heart and Brain: Current Knowledge, Unmet Therapeutic Need, and Future Directions. J Am Heart Assoc 2019;8:e011104.

46. Qiu C, Winblad B, Fratiglioni L. The agedependent relation of blood pressure to cognitive function and dementia. Lancet Neurol 2005;4:487-499.

47. O'Brien JT, Markus HS. Vascular risk factors and Alzheimer's disease. BMC Med 2014;12:218.

48. Bos I, Vos SJB, Schindler SE, et al. Vascular risk factors are associated with longitudinal changes in cerebrospinal fluid tau markers and cognition in preclinical Alzheimer's disease. Alzheimer's \& dementia : the journal of the Alzheimer's Association 2019;15:1149-1159.

49. Salvado G, Brugulat-Serrat A, Sudre $\mathrm{CH}$, et al. Spatial patterns of white matter hyperintensities associated with Alzheimer's disease risk factors in a cognitively healthy middle-aged cohort. Alzheimers Res Ther 2019;11:12.

50. Licher S, Yilmaz P, Leening MJG, et al. External validation of four dementia prediction models for use in the general community-dwelling population: a comparative analysis from the Rotterdam Study. Eur J Epidemiol 2018;33:645-655. 
Associations of LIBRA with structural brain changes and cognition 

Chapter 3

Socioeconomic position, modifiable dementia risk and cognitive decline: results of 12-year the Maastricht Aging Study

Irene Heger, Martin van Boxtel, Kay Deckers, Hans Bosma, Frans Verhey, Sebastian Köhler

Submitted 



\section{Abstract}

Background: Evidence for dementia risk reduction via lifestyle modification is accumulating. People with low socioeconomic status (SES) have increased dementia risk and on average worse health. This study investigated whether the association between a modifiable dementia risk score and rate of cognitive decline differs across SES strata.

Methods: Data were used from the Maastricht Aging Study, a prospective cohort study with a 12-year follow-up $(n=1223)$. The 'LIfestyle for BRAin health' (LIBRA) index was used to assess the modifiable dementia risk fraction. Cognitive performance was measured on the domains of information processing speed, executive functioning and verbal memory function. A SES compound score was calculated from equivalent income and educational level. Linear mixed models were used to study the association between LIBRA, SES and their interaction on the rate of cognitive decline.

Results: Participants in the lowest SES tertile displayed more decline in information processing speed (vs. middle SES: $X^{2}=7.08, P=0.029$; vs. high SES: $X^{2}=9.49, P=0.009$ ) and verbal memory (vs. middle SES: $X^{2}=9.28$, $P<0.001$; vs. high SES: $X^{2}=16.68, P<0.001$ ) compared to their middle- and high-SES counterparts. Higher LIBRA scores were also associated with more decline in information processing speed $\left(X^{2}=12.66, P=0.002\right)$ and verbal memory $\left(\mathrm{X}^{2}=4.63, P=0.032\right)$. No consistent effect modification by SES was found.

Conclusions: Lower SES and higher modifiable dementia risk were associated with faster cognitive decline over 12 years. While associations for the latter did not differ across SES strata, people with lower SES have more room-forlifestyle-improvement, and this should be addressed in future interventions. 


\section{Introduction}

Dementia is characterized by progressive decline of cognitive functions, leading to interferences in daily activities and a loss of autonomy. ${ }^{1}$ Alzheimer's disease is the most common underlying cause of dementia, although mixed aetiologies (e.g., neurodegeneration and vascular damage) often co-exist. ${ }^{1}$ Given the rising number of people with dementia and the ongoing absence of curative treatments, dementia is one of the biggest health challenges of our time. ${ }^{1,2}$ The expected worldwide threefold increase of the number of people with dementia to 152 million in 2050 is particularly attributable to the rising numbers in low-to-middle income countries (LMIC). ${ }^{1,3,4}$ In fact, epidemiological studies have shown stabilization and even decline in incidence rates of dementia in high-income countries (HIC)., 5-9 Possible explanations are the increasing wealth and improved general health in HIC (e.g., improved provision and access to education, better nutrition and cardiovascular risk management) and increasing rates of dementia risk factors in LMIC (e.g., higher prevalence of diabetes mellitus, smoking, hypertension, and obesity). ${ }^{3,5-7}$

Indeed, there is accumulating evidence that modifiable risk factors contribute to cognitive decline and dementia risk, which suggests potential for dementia risk reduction. ${ }^{4,10,11}$ The 2020-update of the Lancet Report on Dementia Prevention, Intervention and Care concluded that around $40 \%$ of all dementia cases is potentially attributable to modifiable risk factors. ${ }^{4}$ The World Health Organization (WHO) identified risk reduction of dementia as one of the action areas for 2017-2025, ${ }^{12}$ which was followed by publishing guidelines for risk reduction of cognitive decline and dementia in $2019 .{ }^{2}$

Besides lifestyle-related risk factors, lower socioeconomic status (SES), in which SES is defined as "social and economic factors that influence which positions individuals or groups will hold within the structure of a society" ${ }^{13}$, has also been associated with an increased risk of cognitive decline and dementia ${ }^{3}$, ${ }^{14-17}$. Additionally, some studies suggest that SES might act as a moderator between lifestyle and cognitive performance. For example, higher adherence to a healthy diet was associated with less cognitive decline over 3-years in older adults (68-84 years) with low SES, compared with high SES (based on income, education and occupation). ${ }^{18} \mathrm{~A}$ prospective cohort study found that financially disadvantaged participants benefitted cognitively the most from living healthy (based on vegetable and fish intake, physical activity, smoking status and light-to-moderate alcohol intake) ${ }^{19}$. Contrary, a prospective cohort 
study from 2019 found that the effect of a modifiable risk score (including lifestyle factors) and cognitive functioning over 10 years was similar across educational groups, another indicator of SES. ${ }^{20}$ In addition, SES measured as income did not modify the lifestyle-intervention effect of the Finnish Geriatric Intervention Study to Prevent Cognitive Impairment and Disability (FINGER) on cognition. ${ }^{21}$

Heterogeneity in these findings could be due to different operationalization of lifestyle and SES (based on a single variable or a compound score) and different populations. More clarity on this matter is important from a public health perspective. It is important to identify and target subgroups that would benefit most from lifestyle interventions and vascular risk management aimed at risk reduction of dementia and to develop more extensive or other types of interventions in specific SES groups, to reach the full potential of primary prevention of dementia.

Therefore, this study aims to contribute to the existing evidence by investigating whether the association between a modifiable dementia risk score and cognitive performance over 12 years is similar across groups with different levels of SES, defined by educational level and income. 


\section{Methods}

\section{Study design}

This study used data from the Maastricht Aging Study (MAAS), a populationbased observational study into successful and pathological cognitive aging. ${ }^{22}$ Data collection for MAAS is ongoing and has to date a fully completed followup period of 12-years. ${ }^{22}$

\section{Participants}

Potential participants for MAAS were randomly drawn from the Research Network Family Medicine Maastricht (RNFM), a registration network of family practices in the Province of Limburg, the Netherlands. ${ }^{23}$ The network consisted of approximately 80000 primary care patients that are representative of the general Dutch population with respect to demographic characteristics. ${ }^{23} \mathrm{~A}$ total of 10396 individuals aged between 24 and 81 years old without morbidity that is known to interfere with cognitive function (e.g., dementia, schizophrenia) received a postal letter with an invitation to participate in MAAS. Interested individuals were screened for exclusion criteria that were not available in the RNFM database (e.g., score of $<24$ on the Mini-Mental State Examination, history of transient ischemic attacks, or brain surgery). ${ }^{22}$ This led to the selection of 1823 Caucasian and Dutch speaking participants who completed the baseline assessment between 1993 and 1995. ${ }^{22,24}$ This study used data from the sample of $\geq 40$ years old at baseline $(n=1223)$ and used the six- and 12-year follow-up assessments as (cognitive) outcome time points.

\section{Modifiable risk factors}

To assess health and lifestyle factors associated with dementia risk, the 'LIfestyle for BRAin health' (LIBRA) index was used. ${ }^{25}$ While most other dementia risk indices are based on single cohort studies and/or include non-modifiable factors such as age, sex or genetic factors, ${ }^{26-30}$ LIBRA is based on a systematic literature review and Delphi consensus ${ }^{25}$ and was designed to capture the modifiable risk fraction of 12 risk and protective factors. LIBRA could therefore be useful as a participant selection tool or as a surrogate outcome measure in lifestyle intervention trials. ${ }^{31,32}$ LIBRA has been associated with cognitive decline, cognitive impairment and dementia risk in several cohorts, particularly in middle-aged and young-old populations. ${ }^{15,}$ 20, 32-37 Presence of LIBRA factors in MAAS (yes/no) was based on self-reported questionnaires and/or clinical data at baseline and defined following established cut-offs. A 
weight (positive for risk factors, negative for protective factors) was assigned to the presence of each LIBRA factor, based on the factor's relative risk. ${ }^{25}$ With the exception of information on adherence to a Mediterranean diet, all LIBRA factors were available in MAAS. The LIBRA total score was calculated for each participant by summation of the individual LIBRA weights (ranging from -4.2 to +12.7 , with higher scores indicating higher dementia risk). Additionally, LIBRA discrete risk groups were calculated based on tertiles (referred to as 'low', 'middle' and 'high' risk). Participants were only included in the analyses if all 11 LIBRA factors were available. Table 1 provides an overview of the LIBRA factors, assigned weights and operationalization in MAAS. 


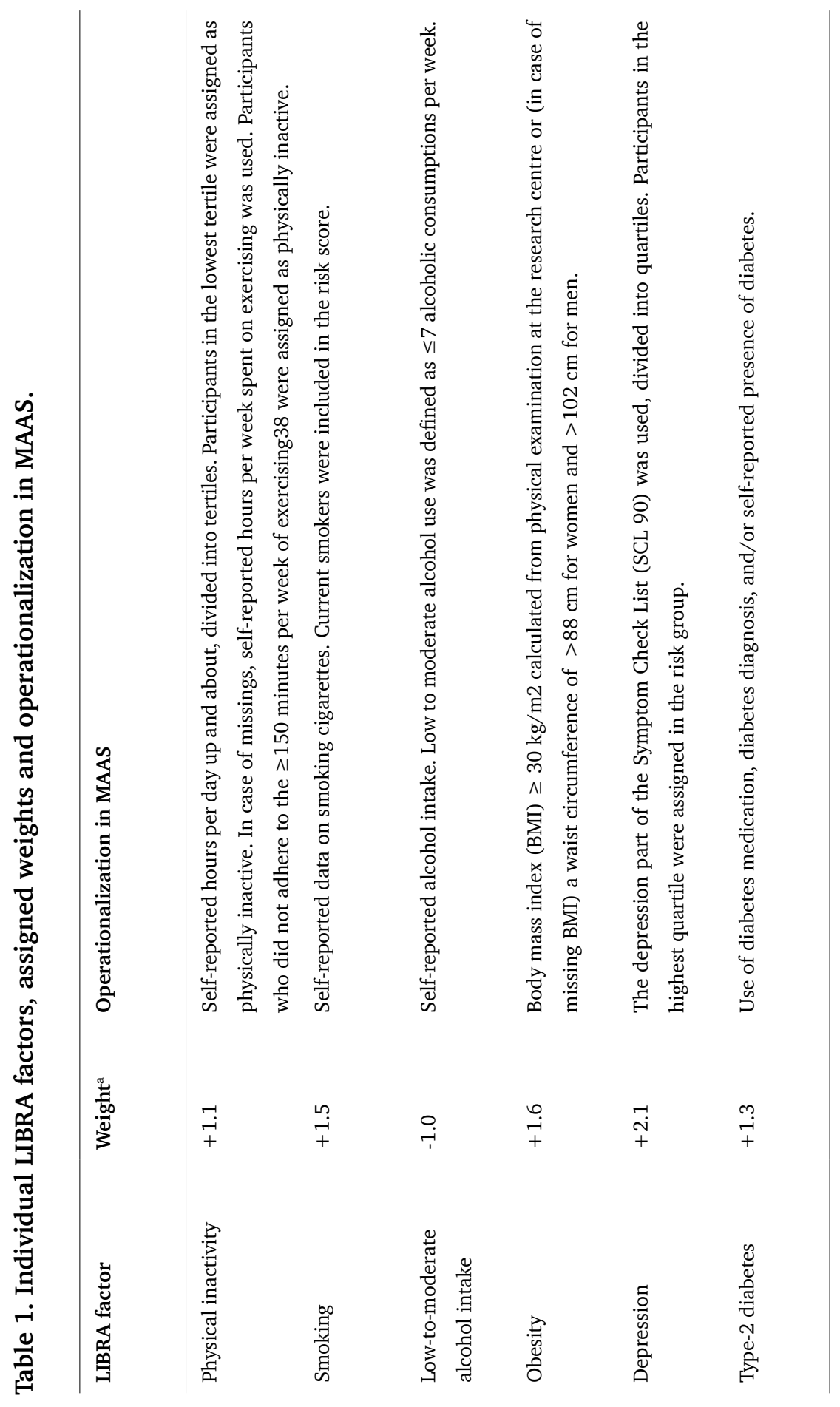




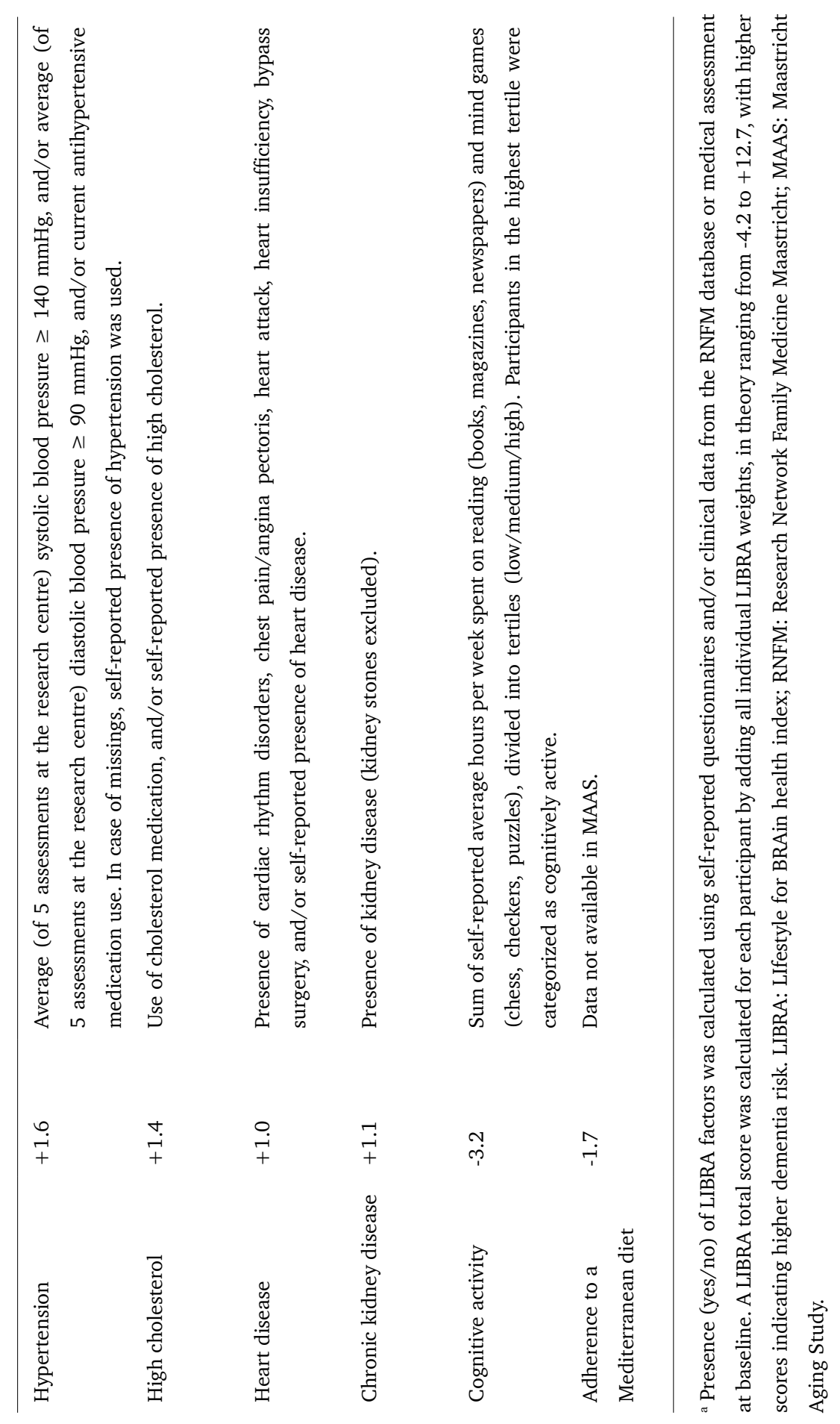




\section{Cognitive outcomes}

Cognitive performance was assessed with a neuropsychological assessment administered by trained personnel at baseline, six- and 12-years follow-up. The Verbal Learning Test (VLT) was used to assess verbal memory. A total of 15 meaningful words were successively presented after which a participant was asked to recall as many words as possible, with five consecutive trials. Number of recalled words after 20 minutes assessed delayed recall and was used as an outcome measure in this study. ${ }^{39}$ To assess information processing speed, the written version of the Letter-Digit Substitution Test (LDST) was used. In this test, participants were given 90 seconds to match as many letters as possible to the given numbers following the key at the top of the test sheet. ${ }^{40}$ The Stroop Colour-Word Test (SCWT) was used to assess executive functioning. Three cards were consecutively presented to participants, each displaying 100 colour names (card one), coloured patches (card two) and colour names printed in another colour's ink (card three). Cognitive interference, the time needed to ignore irrelevant but very outstanding (verbal) information in favour of less salient information (colour naming) in card three, was used to assess executive functioning. ${ }^{41}$ Participants were only included in the study sample if at least one of the three cognitive tests was administered at baseline.

\section{Socioeconomic status}

SES was assessed using a compound score of household income and educational level. Income was measured by self-reported net month income of the household in Euro, with 12 answering options ranging from less than 680 euro to more than 2949 euro. In MAAS, income was measured from the six-year followup and available for $712(58.2 \%)$ participants of the study sample. In order to account for the household size living from a household income, income was then recoded into a continuous equivalent income measure by using the Organisation for Economic Co-operation and Development (OECD) square root scale. ${ }^{42}$ The midpoint of each category of income was taken and divided by the squared root of the number of people that this income should provide for. Educational level was measured using the highest finalized educational degree of a participant in eight categories (primary school, low vocational education, intermediate secondary education, intermediate vocational or higher secondary education, higher vocational education, university, other), assessed at baseline. To compute an overall compound score of SES, equivalent income and educational level were standardized and averaged and subsequently categorized into tertiles. 


\section{Statistical analysis}

$\mathrm{Chi}^{2}$ tests and t-tests were used to nvestigate differences in socio-demographics, LIBRA profile and cognitive performance between the baseline study sample and those excluded (i.e., < 11 LIBRA factors measured). ANOVA's and chi $^{2}$ tests were used to study differences in the aforementioned determinants between the three SES tertiles of the baseline study sample (low, middle and high SES). Linear mixed models were used to study the association between LIBRA, SES and cognition over time, adjusted for baseline age, age ${ }^{2}$, and sex. To correct for skewness, data on the VLT delayed recall was squared and data on the SCWT interference score was log-transformed. As suggested by likelihood ratio tests, the models included a random intercept, random slope and unstructured covariance matrix. Interaction terms were added to the models to investigate whether participants with different LIBRA scores (time*LIBRA; using dummy variables for the two follow-ups of time) or SES scores (time*SES; dummy's for middle and high SES) differ in the rate of change of cognition over time. The interaction terms were tested using a Wald test which yields a chi ${ }^{2}$ statistic with 2 degrees of freedom. Next, three-way interactions (time*LIBRA*SES) were added to the models to study the effect of SES on the association between LIBRA and cognition over time, followed by stratified analyses in case of statistical significance. Analyses were first performed for continuous LIBRA scores, and then repeated using LIBRA tertiles (low-risk, middle-risk, high-risk). Two inverse probability weights were used to reduce selection bias. First, an attrition weight was used. While the mixed model assumes data to be missing at random, conditional on included covariates, the weighting further minimizes bias due to selective dropout. The attrition weight was based on probit regression including demographic and health-related factors associated with missingness. Second, a sampling weight using age, gender and level of occupational achievement as predictors was added to weight the estimates back to the RNFM source population participants were recruited from. All analyses were performed in Stata 13.1 (StataCorp, College Station, TX, USA) with a level of statistical significance set a $P<0.05$ in two-tailed tests.

\section{Data availability statement}

The data underlying this article will be shared on reasonable request to the corresponding author. 


\section{Results}

\section{Participants}

A flowchart of the recruitment process and selection of the study sample is shown in Figure 1. Table 2 presents the baseline characteristics of the study sample by SES groups (low, middle, high). Participants in the lowest SES group were older, more often female, had a higher (i.e., unhealthier) LIBRA score and lower performance on cognition tasks. In contrast, the protective factor low-to-moderate alcohol use was more common in the lowest SES group and participants in the high SES group were more often physically inactive compared to their low- and middle counterparts.
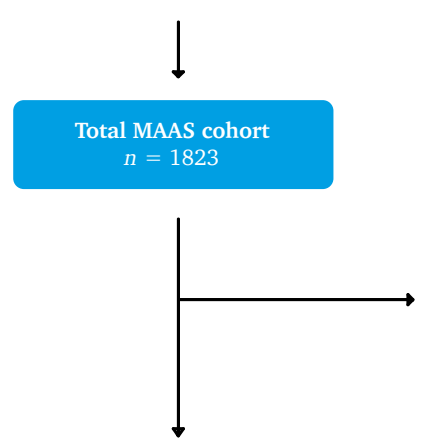

Excluded from analyses:

- Excluded by design (age $\leq 40$ years at baseline):

$n=525$

- Excluded due to $\geq 1$ LIBRA factors missing:

$n=72$

Baseline study sample $n=1223$

Study sample 6 year follow-up $n=928$

Study sample 12 year follow-up $n=755$

Figure 1. Flowchart of the recruitment process and selection of study sample. RNFM: Research Network Family Medicine Maastricht; MAAS: Maastricht Aging Study; LIBRA: LIfestyle for BRAin health index.
} 
Table 2. Characteristics of the baseline study population $(n=1223)$ by socioeconomic status.

\begin{tabular}{lrrrr}
\hline Variables $^{\mathrm{a}}$ & Low SES & Middle SES & High SES \\
& $\mathrm{n}=419(34.3 \%)$ & $\mathrm{n}=402(32.9 \%)$ & $\mathrm{n}=402(32.9 \%)$ & $P$ \\
\hline
\end{tabular}

Demographics

Age; mean (SD) $\quad 62.4(11.1) 40-82 \quad 57.9(10.6) 40-79 \quad 56.3(12.1) 40-82 \quad<0.001$

range

Female; n (\%)

247 (59.0\%)

199 (49.5\%)

$156(38.8 \%)<0.001$

Educational level;

$<0.001$

n (\%)

Low

$413(98.6 \%)$

$114(28.4 \%)$

$4(1.0 \%)$

Middle

$6(1.4 \%)$

$284(70.7 \%)$

$170(42.3 \%)$

High

$0(0 \%)$

$4(1.0 \%)$

$228(56.7 \%)$

LIBRA health

and lifestyle

factors; $n(\%)$

Hypertension

$200(47.9 \%)$

139 (34.6\%)

$140(34.7 \%) \quad<0.001$

Diabetes

$35(8.4 \%)$

$18(4.5 \%)$

$16(4.0 \%)$

0.011

Hypercholestero-

$51(12.2 \%)$

$56(13.9 \%)$

$50(12.4 \%)$

0.724

lemia

Coronary heart

$66(15.8 \%)$

$61(15.2 \%)$

$60(14.9 \%)$

0.935

disease

Table continues on next page. 


\begin{tabular}{|c|c|c|c|c|}
\hline $\begin{array}{l}\text { Chronic kidney } \\
\text { disease }\end{array}$ & $21(5.0 \%)$ & $17(4.2 \%)$ & $18(4.5 \%)$ & 0.855 \\
\hline Obesity & $114(27.3 \%)$ & 79 (19.7\%) & $63(15.6 \%)$ & $<0.001$ \\
\hline Smoking & $110(26.3 \%)$ & 99 (24.6\%) & $110(27.3 \%)$ & 0.684 \\
\hline Cognitive activity & $111(26.6 \%)$ & $113(28.1 \%)$ & $141(35.0 \%)$ & 0.020 \\
\hline Physical inactivity & 125 (29.9\%) & $136(33.8 \%)$ & $211(52.4 \%)$ & $<0.001$ \\
\hline $\begin{array}{l}\text { Low-to-moderate } \\
\text { alcohol use }\end{array}$ & $296(70.8 \%)$ & 259 (64.4\%) & 201 (49.9\%) & $<0.001$ \\
\hline $\begin{array}{l}\text { High depressive } \\
\text { symptoms }\end{array}$ & $114(27.3 \%)$ & $91(22.6 \%)$ & $75(18.6 \%)$ & 0.013 \\
\hline $\begin{array}{l}\text { LIBRA score; } \\
\text { mean (SD) range }\end{array}$ & $\begin{array}{r}1.42(2.33) \\
-4.2 \text { to }+9.2\end{array}$ & $\begin{array}{r}1.05(2.27) \\
-4.2 \text { to }+7.7\end{array}$ & $\begin{array}{l}0.94(2.40) \\
-4.2 \text { to }+7.4\end{array}$ & 0.008 \\
\hline $\begin{array}{l}\text { Cognitive } \\
\text { function score }{ }^{\mathrm{b}} \text {; } \\
\text { mean (SD) }\end{array}$ & & & & \\
\hline $\begin{array}{l}\text { Information } \\
\text { processing speed }\end{array}$ & $39.05(9.86)$ & $45.54(9.56)$ & $50.61(10.05)$ & $<0.001$ \\
\hline $\begin{array}{l}\text { Executive } \\
\text { functioning }\end{array}$ & $60.19(30.95)$ & 47.89 (18.69) & $43.26(18.44)$ & $<0.001$ \\
\hline Verbal memory & $8.41(2.90)$ & $9.05(2.84)$ & $9.70(3.09)$ & $<0.001$ \\
\hline
\end{tabular}

\footnotetext{
a Numbers and percentages do not add up, due to missing values. ${ }^{\mathrm{b}}$ Information processing speed was assessed with the LDST (higher scores reflect better performance), executive functioning with the SCWT interference score (lower scores reflect better performance), and verbal memory with the delayed recall score of the VLT (higher scores reflect better performance). SES: socioeconomic status (compound score of educational level and equivalent month income); SD: standard deviation; LIBRA: LIfestyle for BRAin health index.
} 
Lifestyle and cognitive decline

At baseline, higher LIBRA scores were associated with lower scores on information processing speed $(\mathrm{B}=-0.37,95 \% \mathrm{CI}=-0.58$ to $-0.16, P<0.001$; results in this paragraph not tabulated) and executive functioning $(B=0.02$, $95 \% \mathrm{CI}=0.01$ to $0.04, P<0.001)$, but not on verbal memory $(B=-1.01$, $95 \% \mathrm{CI}=-2.15$ to $0.12, P=0.081$ ). In the prospective analyses, a higher LIBRA score was associated with faster decline in information processing speed (Wald test for overall interaction: $\mathrm{X}^{2}=12.66, \mathrm{df}=2, P=0.002$; 6-year LIBRA*time interaction: $\mathrm{B}=-0.25, \quad \mathrm{X}^{2}=7.39, \quad \mathrm{df}=1, \quad P=0.007 ; \quad 12$-year LIBRA*time interaction: $\left.\mathrm{B}=-0.47, \mathrm{X}^{2}=11.64, \mathrm{df}=1, P=0.001\right)$. There was no association between the continuous LIBRA and decline in executive functioning (Wald test for overall interaction: $\mathrm{X}^{2}=0.03, \mathrm{df}=2, P=0.987 ; 6$-year LIBRA*time interaction $\mathrm{B}=-0.0004, \mathrm{X}^{2}=0.00, \mathrm{df}=1, P=0.947 ; 12$-year LIBRA*time interaction: $\mathrm{B}=-0.002$, $\left.\mathrm{X}^{2}=0.02, \mathrm{df}=1, P=0.875\right)$. The difference for verbal memory from baseline to 6-year follow-up $\left(B=-1.52, X^{2}=4.63, \mathrm{df}=1, P=0.032\right)$ was attenuated at 12-year follow-up $\left(\mathrm{B}=-0.67, \mathrm{X}^{2}=0.45, \mathrm{df}=1, P=0.502\right)$, and thus there was no overall association found $\left(\mathrm{X}^{2}=4.72, \mathrm{df}=2, P=0.094\right)$.

Table 3 presents the results of the comparison of the middle- and high-risk groups with the low-risk group, based on LIBRA tertiles. Faster decline in information processing speed was observed in the high-risk group compared to the low-risk group over 12-years follow-up. There was an increase (not tabulated) in verbal memory score from baseline to 6-year follow-up. This improvement in performance was lower in the high-risk group than in the low-risk group. No effect was observed from 6-years to 12-years follow-up. No differences over time were observed in executive functioning and there were no differences in cognition scores over time between the middle- and high-risk groups. The trajectories over time (baseline until 12-year follow-up) of the cognitive tests for the three LIBRA risk groups are displayed in Figure 2. 


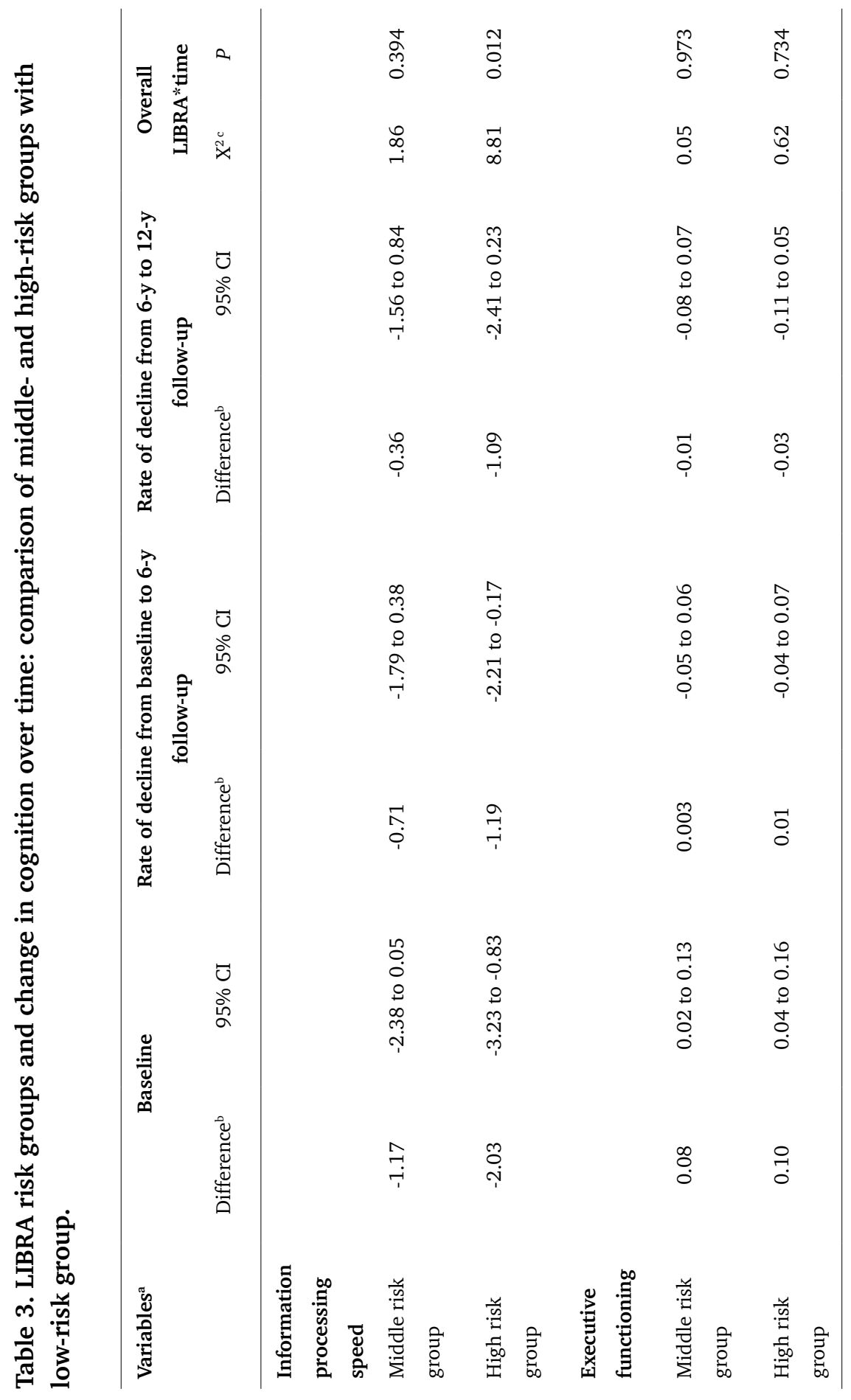




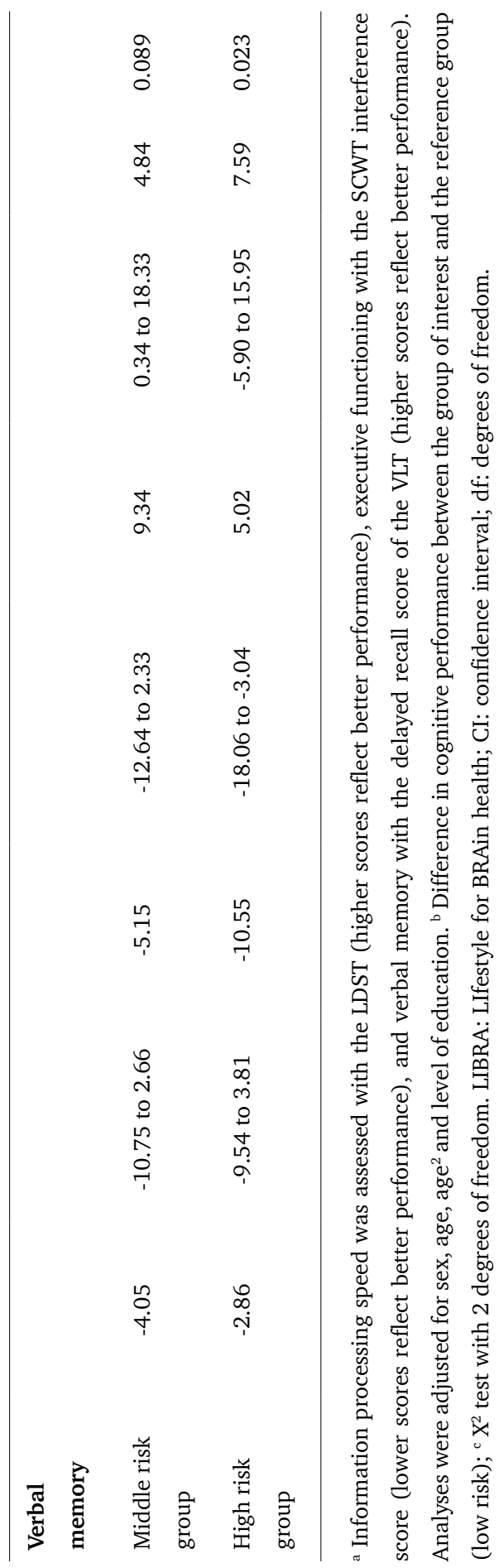



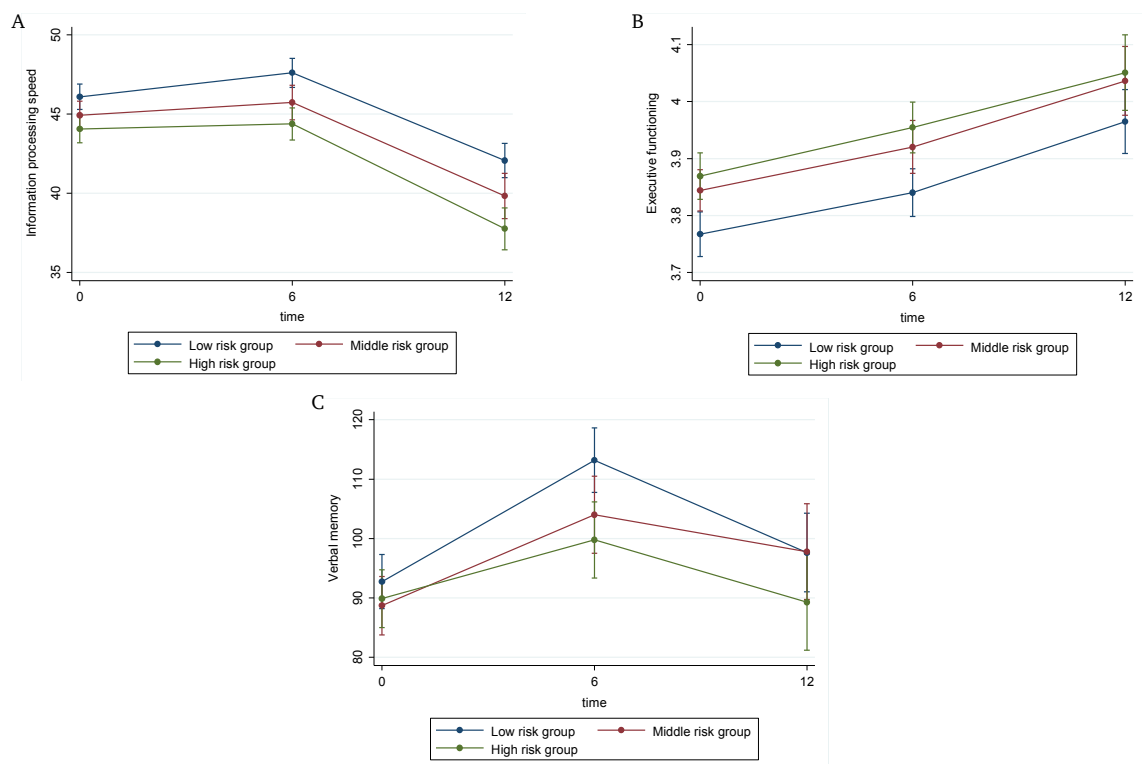

Figure 2. Estimated marginal means of cognitive trajectories over time for the LIBRA risk groups. ${ }^{a}$ Information processing speed, assessed with the LDST (higher scores reflect better performance);

b Executive functioning, assessed with the SCWT interference score (log-transformed; lower scores reflect better performance); ${ }^{\text {c }}$ Verbal memory, assessed with the delayed recall score of the VLT (squared; higher scores reflect better performance). Adjusted for sex, age, age ${ }^{2}$ and level of education. LIBRA: LIfestyle for BRAin health index. 
SES and cognitive decline

Table 4 presents the results of the comparison between the three SES groups, based on SES tertiles. Participants in the middle- and high SES groups had less decline in information processing speed and verbal memory (except middle SES vs. low SES in the 6-y follow-up of verbal memory) compared to participants in the low SES group. There was no difference in decline between the middle and high SES groups. There was also no difference in executive functioning observed over time between the three SES groups. Figure 3 displays the cognitive trajectories of the three SES groups over time.

Differences in socioeconomic status

None of the three-way interaction models showed a modifying effect of SES on the relationship between continuous LIBRA and cognition. When categorizing LIBRA into three risk groups (tertiles), a modifying effect of SES was found in executive functioning. With increasing SES, the middle-risk group needed less time to complete this task compared to the low-risk group from baseline to 6-year follow-up ( $\mathrm{B}=-0.05,95 \% \mathrm{CI}=-0.10$ to $-0.01, P=0.013)$. This effect was attenuated at 12 -year follow-up ( $\mathrm{B}=0.002,95 \% \mathrm{CI}=-0.08$ to $0.08, P=0.970$ ) and no effects were found for the high-risk group compared to the low risk group (baseline to 6-year: $\mathrm{B}=0.01,95 \% \mathrm{CI}=-0.04$ to $0.06, P=0.749$; 6 to 12 year follow-up: $\mathrm{B}=0.08,95 \% \mathrm{CI}=-0.01$ to $0.17, P=0.074$ ). 


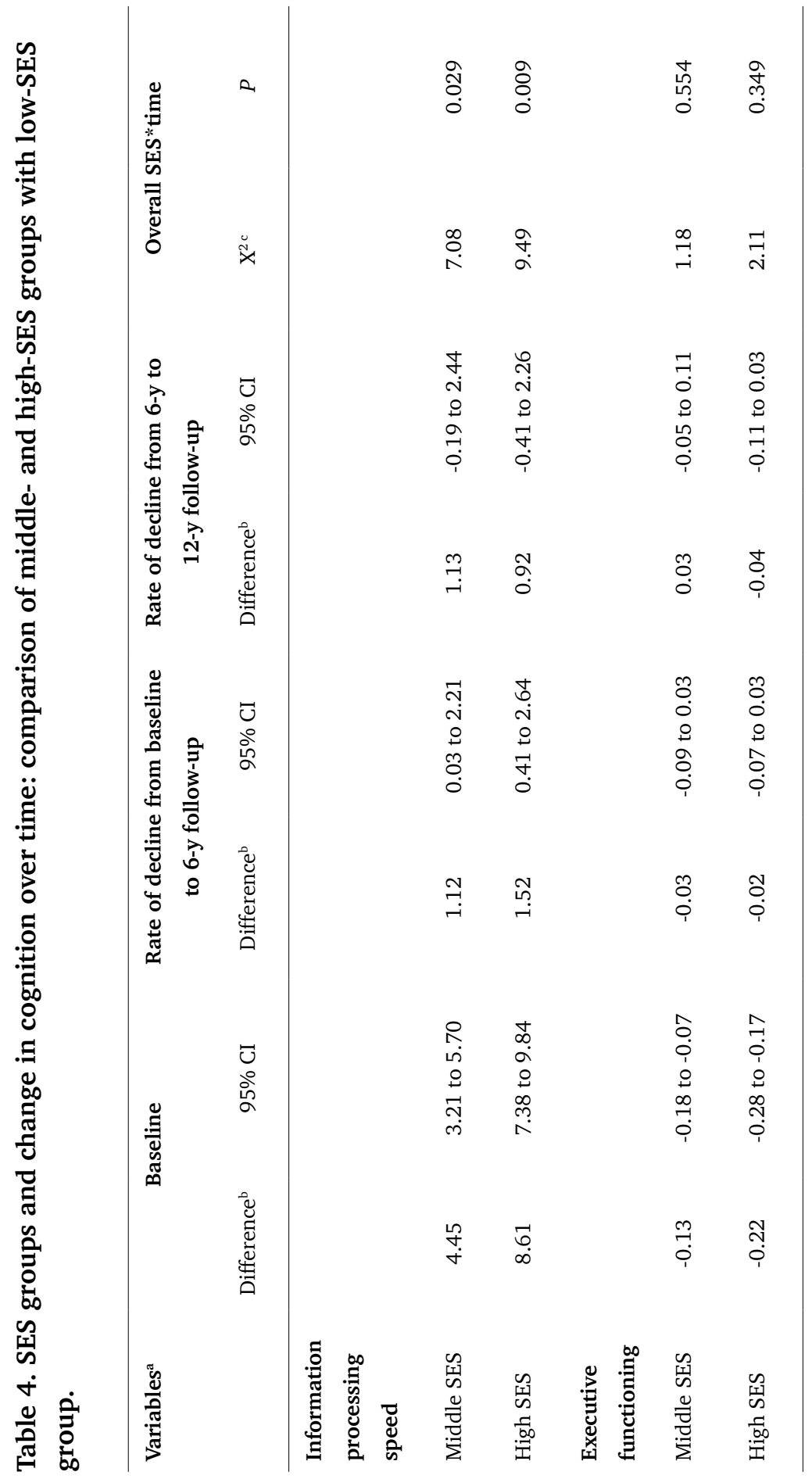




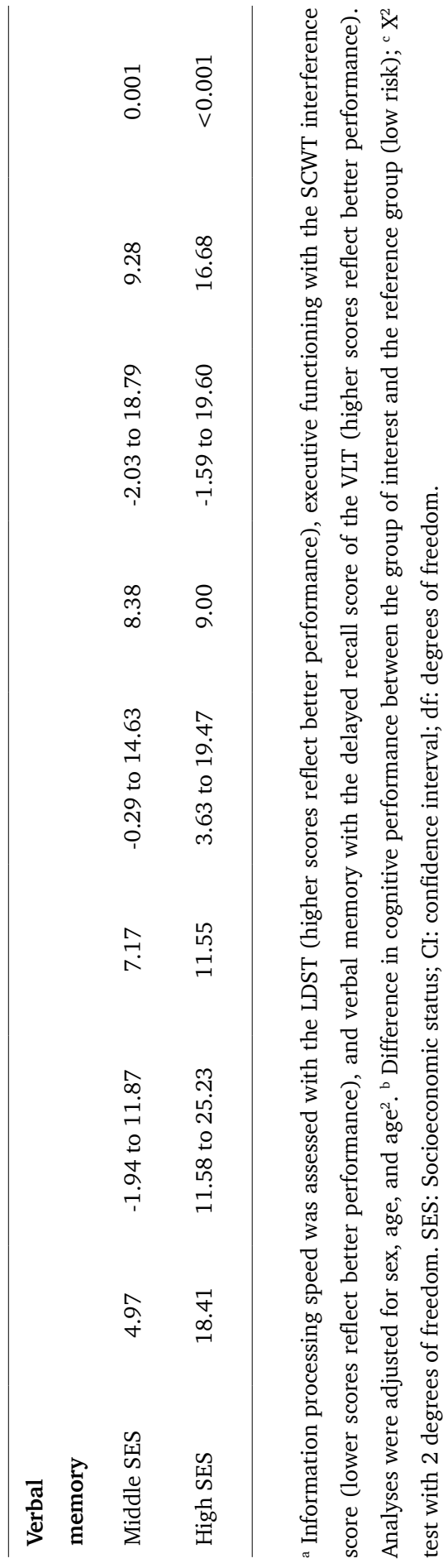


A

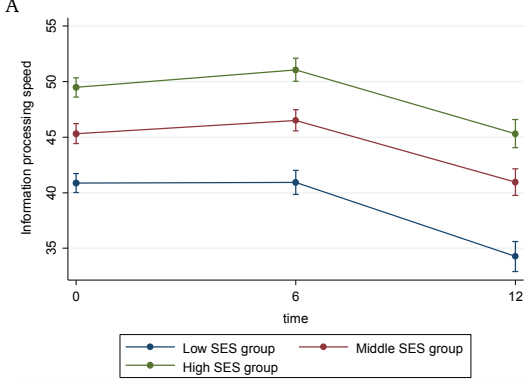

B

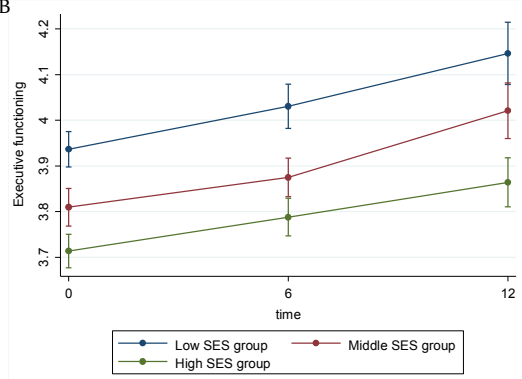

C

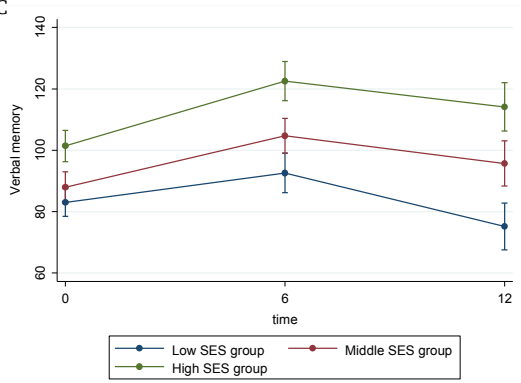

Figure 3. Estimated marginal means of cognitive trajectories of the SES groups over time.

a Information processing speed, assessed with the LDST (higher scores reflect better performance);

b Executive functioning, assessed with the SCWT interference score (log-transformed; lower scores reflect better performance); " Verbal memory, assessed with the delayed recall score of the VLT (squared; higher scores reflect better performance). Adjusted for sex, age and age ${ }^{2}$. SES: socioeconomic status (assessed using a compound score of equivalent income and/or educational level). 
Sensitivity analyses

We performed sensitivity analyses in which the three-way interaction models were performed including education or income separately (in tertiles), instead of the combined SES compound score. Results for the continuous LIBRA score did not change, meaning that none of these models showed a modifying effect of education or income on the relationship between LIBRA and cognition. When categorizing LIBRA into three risk groups (tertiles), the modifying effect of the original SES model in executive functioning no longer appeared $(B=-0.11$, $95 \% \mathrm{CI}=-0.26$ to $0.03, P=0.120$ ). A modifying effect of income was found in information processing speed, in which participants within the highest income group with the highest (i.e., most unhealthy) risk profile performed worse from baseline to 6-year follow-up compared to participants within the lowest income group ( $\mathrm{B}=-2.87,95 \% \mathrm{CI}=-5.69$ to $-0.05, P=0.046$ ). 


\section{Discussion}

This study prospectively examined the association between the modifiable dementia risk score LIBRA and cognitive decline across different SES strata over 12-years in a cognitively healthy population aged 40 years and above. Results show that higher LIBRA scores, reflecting a less brain-healthy lifestyle, predicted more decline in information processing speed and verbal memory function. Participants in the lowest SES group displayed more decline in information processing speed and verbal memory function compared to the higher SES groups. No consistent effect modification was found of SES on the association between LIBRA and rate of cognitive decline.

Higher modifiable dementia risk was associated with faster decline in cognitive performance, which is in line with previous work. ${ }^{15}, 20,32,33,36,37$ The finding that the improved performance in verbal memory score from baseline to 6-year follow-up was lower in the high-risk group than in the low-risk group indicated that the former benefitted less from the practice effect that is commonly observed in repeated assessment of verbal memory ${ }^{43}$. Possible pathophysiological mechanisms by which the different LIBRA factors affect cognition are neurodegenerative processes ${ }^{44}$, cerebrovascular pathology ${ }^{45}$, atherosclerotic burden ${ }^{46}$ metabolic and inflammatory processes 25 and enhanced brain reserve, leading to delayed cognitive decline. ${ }^{47}$

No consistent effect modification of SES was found, which is in line with previous work investigating LIBRA and cognitive performance in different educational strata. ${ }^{20}$ It should be noted that analyses were conducted at the level of the LIBRA total score, which takes all 11 LIBRA factors into account, and that differences in SES for individual risk or protective factors (e.g., hypertension, physical inactivity) was not assessed and could have been present. ${ }^{20}$ Yet, the used methods served our purpose since LIBRA aims to identify the degree of change based on the total modifiable risk spectrum. These findings suggest that modifiable lifestyle and health factors are similarly associated with cognitive decline across SES strata. This finding seems to make a case for universal dementia risk reduction across all SES levels. However, our study also shows that participants with lower SES have a poorer health and lifestyle status and faster decline in cognitive performance. Thus, the modifiable risk fraction (i.e., the roomfor-improvement) is higher in people with low SES. Consequently, health inequalities will increase further if these high-risk groups -which are often 
also hard-to-reach ${ }^{48-50}$ - are missed in the development and out-roll of future interventions.

Strengths of this study include the prospective design, which allowed us to assess exposure to LIBRA factors years prior to our cognitive outcome measures and to analyse serial assessment of cognitive functioning with a comprehensive neuropsychological test battery, and the large sample size. Further, this study is strengthened by using a modifiable dementia risk score that is developed based on an extensive systematic literature review and Delphi consensus study ${ }^{25}$. Last, we used a resource-based SES operationalization ${ }^{42}$ not only based on educational level, which is also a proxy for cognitive reserve ${ }^{14}$, but also on an economic (income) measure that accounts for household size. This study, however, also has some limitations that need to be addressed. First, dropout of the study at 6- and 12-years follow-up could have led to selection bias. This is an unfortunate but common phenomenon in ageing studies. Still, the response rate was quite high for a prospective study $(71.7 \%$ from baseline to 6-year follow-up, and $81.4 \%$ from 6 to 12-year) and the use of linear mixed models allowed us to use maximum likelihood estimations ("imputation") of missing outcome data to minimize selection bias. Furthermore, although the use of a SES compound score of education and equivalent income should foremost be seen as a strength of this study, it should be noted that there was no data on equivalent income in $41.8 \%(n=511)$ of the participants, which resulted in a SES score based on only educational level in this group. Further, the use of self-reported measures to calculate most of the LIBRA factors and net month income could have led to recall bias or socially desirable responses and could have affected the predictive accuracy. Still, most LIBRA factors were operationalized based on a combination of self-report and a clinical measure (e.g., hypertension), diagnosis and/or registered medication use (e.g., diabetes, heart disease). Last, although LIBRA assumes all individual factors to have an added predictive value, the underlying mechanisms by which the individual LIBRA factors affect cognition could interact and this was not considered in the design of the LIBRA score.

\section{Conclusion}

A higher modifiable risk score and SES predicted cognitive decline over 12 years. Although modifiable factors were equally associated with cognitive decline across SES strata, the modifiable risk fraction is higher in people with low SES. It is thus important to tailor interventions towards the needs, wishes 
Chapter 3

and barriers of subgroups, defined by SES and/or risk status. It appears that the largest prevention gain is to be earned in lower SES strata. 


\section{References}

1. World Health Organization. Factsheet Dementia. https://www.who.int/newsroom/fact-sheets/detail/dementia (9-22021, date last accessed).

2. Risk reduction of cognitive decline and dementia: WHO guidelines. 2019, World Health Organization: Geneva.

3. Hachinski V, Einhäupl $\mathrm{K}$, Ganten $\mathrm{D}$, et al. Preventing dementia by preventing stroke: The Berlin Manifesto. Alzheimers Dement 2019;15:961-84.

4. Livingston G, Huntley J, Sommerlad A, et al. Dementia prevention, intervention, and care: 2020 report of the Lancet Commission. Lancet 2020;396:413-46.

5. Roehr S, Pabst A, Luck T, Riedel-Heller SG. Is dementia incidence declining in high-income countries? A systematic review and meta-analysis. Clin Epidemiol 2018;10:1233-47.

6. Prince $\mathrm{M}$, Ali G-C, Guerchet $\mathrm{M}$, Prina AM, Albanese E, Wu Y-T. Recent global trends in the prevalence and incidence of dementia, and survival with dementia. Alzheimers Res Ther 2016;8:23-23.

7. Satizabal CL, Beiser AS, Chouraki V, Chêne G, Dufouil C, Seshadri S. Incidence of Dementia over Three Decades in the Framingham Heart Study. NEJM 2016;374:523-32.

8. Wolters FJ, Chibnik LB, Waziry $\mathrm{R}$, et al. Twenty-seven-year time trends in dementia incidence in Europe and the United States: The Alzheimer Cohorts Consortium. Neurology 2020;95:e519-e31.
9. Matthews FE, Stephan BC, Robinson L, et al. A two decade dementia incidence comparison from the Cognitive Function and Ageing Studies I and II. Nat Commun 2016;7:11398.

10. Norton S, Matthews FE, Barnes DE, Yaffe K, Brayne C. Potential for primary prevention of Alzheimer's disease: an analysis of population-based data. Lancet Neurol 2014;13:788-94.

11. Lincoln P, Fenton $K$, Alessi $C$, et al. The Blackfriars Consensus on brain health and dementia. Lancet 2014;383:1805-06.

12. Global action plan on the public health response to dementia 2017-2025. 2017, World Health Organization: Geneva.

13. Krieger $\mathrm{N}$, Williams $\mathrm{DR}$, Moss NE. Measuring social class in US public health research: concepts, methodologies, and guidelines. Annu Rev Public Health 1997; 18:341-78.

14. George KM, Lutsey PL, Kucharska-Newton A, et al. Life-Course Individual and Neighborhood Socioeconomic Status and Risk of Dementia in the Atherosclerosis Risk in Communities Neurocognitive Study. Am J Epidemiol 2020;189:113442.

15. Deckers $\mathrm{K}$, Cadar $\mathrm{D}$, van Boxtel MPJ, Verhey FRJ, Steptoe A, Kohler S. Modifiable Risk Factors Explain Socioeconomic Inequalities in Dementia Risk: Evidence from a Population-Based Prospective Cohort Study. $J$ Alzheimers Dis 2019;71:549-57.

16. Yaffe K, Falvey C, Harris TB, et al. Effect 
of socioeconomic disparities on incidence of dementia among biracial older adults: prospective study. BMJ 2013;347:f7051.

17. Zeki Al Hazzouri A, Haan MN, Kalbfleisch JD, Galea S, Lisabeth LD, Aiello AE. Life-course socioeconomic position and incidence of dementia and cognitive impairment without dementia in older Mexican Americans: results from the Sacramento area Latino study on aging. Am J Epidemiol 2011;173:1148-58.

18. Parrott MD, Shatenstein B, Ferland G, et al. Relationship between diet quality and cognition depends on socioeconomic position in healthy older adults. $J$ Nutr 2013;143:1767-73.

19. Weng PH, Chen JH, Chiou JM, et al. The effect of lifestyle on late-life cognitive change under different socioeconomic status. PLoS One 2018;13:e0197676.

20. Deckers K, Nooyens A, van Boxtel M, Verhey F, Verschuren M, Kohler S. Gender and Educational Differences in the Association between Lifestyle and Cognitive Decline over 10 Years: The Doetinchem Cohort Study. $J$ Alzheimers Dis 2018;70:S31-S41.

21. Rosenberg A, Ngandu T, Rusanen M, et al. Multidomain lifestyle intervention benefits a large elderly population at risk for cognitive decline and dementia regardless of baseline characteristics: The FINGER trial. Alzheimer's \& dementia : the journal of the Alzheimer's Association 2018;14:263-70.

22. Jolles J, Houx PJ, van Boxtel MPJ, Ponds RWHM, Maastricht Aging Study. 1995, Neuropsych Publishers: Maastricht.
23. Metsemakers JF, Höppener P, Knottnerus JA, Kocken RJ, Limonard CB. Computerized health information in The Netherlands: a registration network of family practices. $\mathrm{Br}$ J Gen Pract 1992;42:102-6.

24. van Boxtel MP, Buntinx F, Houx PJ, Metsemakers JF, Knottnerus A, Jolles J. The relation between morbidity and cognitive performance in a normal aging population. J Gerontol A Biol Sci Med Sci 1998;53:M147-54.

25. Deckers K, van Boxtel MP, Schiepers OJ, et al. Target risk factors for dementia prevention: a systematic review and Delphi consensus study on the evidence from observational studies. Int $J$ Geriatr Psychiatry 2015;30:234-46.

26. Stephen R, Liu Y, Ngandu T, et al. Associations of CAIDE Dementia Risk Score with MRI, PIB-PET measures, and cognition. J Alzheimers Dis 2017;59:695705.

27. Kivipelto $M$, Ngandu $T$, Laatikainen $T$, Winblad B, Soininen H, Tuomilehto J. Risk score for the prediction of dementia risk in 20 years among middle aged people: a longitudinal, population-based study. Lancet Neurol 2006;5:735-41.

28. Reitz C, Tang MX, Schupf N, Manly JJ, Mayeux R, Luchsinger JA. A summary risk score for the prediction of Alzheimer disease in elderly persons. Arch Neurol 2010;67:835-41.

29. Exalto LG, Quesenberry CP, Barnes D, Kivipelto M, Biessels GJ, Whitmer RA. Midlife risk score for the prediction of dementia four decades later. Alzheimers Dement 2014;10:562-70. 
30. Cherbuin N, Shaw ME, Walsh E, Sachdev P, Anstey KJ. Validated Alzheimer's Disease Risk Index (ANU-ADRI) is associated with smaller volumes in the default mode network in the early 60s. Brain Imaging Behav 2019;13:65-74.

31. Coley N, Hoevenaar-Blom MP, van Dalen JW, et al. Dementia risk scores as surrogate outcomes for lifestyle-based multidomain prevention trials-rationale, preliminary evidence and challenges. Alzheimers Dement 2020;16:1674-85.

32. Deckers $\mathrm{K}$, Köhler $\mathrm{S}$, Ngandu $\mathrm{T}$, et al. Quantifying dementia prevention potential in the FINGER randomized controlled trial using the LIBRA prevention index. Alzheimers Dement 2021.

33. Vos SJB, van Boxtel MPJ, Schiepers OJG, et al. Modifiable Risk Factors for Prevention of Dementia in Midlife, Late Life and the Oldest-Old: Validation of the LIBRA Index. $J$ Alzheimers Dis 2017;58:537-47.

34. Deckers K, Kohler S, van Boxtel M, Verhey F, Brayne C, Fleming J. Lack of associations between modifiable risk factors and dementia in the very old: findings from the Cambridge City over-75s cohort study. Aging Ment Health 2017;22:1272-78.

35. Pons A, LaMonica HM, Mowszowski L, Kohler S, Deckers K, Naismith SL. Utility of the LIBRA Index in Relation to Cognitive Functioning in a Clinical Health Seeking Sample. J Alzheimers Dis 2018;62:373-84.

36. Schiepers OJG, Kohler S, Deckers K, et al. Lifestyle for Brain Health (LIBRA): a new model for dementia prevention. Int $J$ Geriatr Psychiatry 2018;33:167-75.

37. Deckers K, Barbera M, Köhler S, et al. Long-term dementia risk prediction by the LIBRA score: A 30-year follow-up of the CAIDE study. Int $J$ Geriatr Psychiatry 2020;35:195-203.

38. Weggemans RM, Backx FJG, Borghouts L, et al. The 2017 Dutch Physical Activity Guidelines. Int $J$ Behav Nutr Phys Act 2018;15:58.

39. Van der Elst W, van Boxtel MP, van Breukelen GJ, Jolles J. Rey's verbal learning test: normative data for 1855 healthy participants aged 24-81 years and the influence of age, sex, education, and mode of presentation. $J$ Int Neuropsychol Soc 2005;11:290-302.

40. van der Elst W, van Boxtel MP, van Breukelen GJ, Jolles J. The Letter Digit Substitution Test: normative data for 1,858 healthy participants aged 24-81 from the Maastricht Aging Study (MAAS): influence of age, education, and sex. $J$ Clin Exp Neuropsychol 2006;28:9981009.

41. Van der Elst W, Van Boxtel MP, Van Breukelen GJ, Jolles J. The Stroop colorword test: influence of age, sex, and education; and normative data for a large sample across the adult age range. Assessment 2006;13:62-79.

42. OECD, Divided We Stand: Why Inequality Keeps Rising. 2011.

43. Gavett BE, Gurnani AS, Saurman JL, et al. Practice Effects on Story Memory and List Learning Tests in the Neuropsychological Assessment of Older Adults. PLoS One 
2016;11:e0164492.

44. O'Brien JT, Markus HS. Vascular risk factors and Alzheimer's disease. BMC Med 2014;12:218.

45. Wolters FJ, Zonneveld HI, Hofman A, et al. Cerebral Perfusion and the Risk of Dementia: A Population-Based Study. Circulation 2017;136:719-28.

46. Qiu C, Winblad B, Fratiglioni L. The agedependent relation of blood pressure to cognitive function and dementia. Lancet Neurol 2005;4:487-99.

47. Stern Y. Cognitive reserve in ageing and Alzheimer's disease. Lancet Neurol 2012;11:1006-12.

48. Luten KA, Dijkstra A, de Winter AF, Reijneveld SA. Developing a communitybased intervention for Dutch older adults in a socioeconomically disadvantaged community. Health Promot Int 2018.

49. Lafortune L, Martin S, Kelly S, et al. Behavioural Risk Factors in Mid-Life Associated with Successful Ageing, Disability, Dementia and Frailty in Later Life: A Rapid Systematic Review. 2016. 11, 1-34 DOI: 10.1371/journal. pone.0144405.

50. Steyaert J, Deckers K, Smits C, et al. Putting primary prevention of dementia on everybody's agenda. Aging Ment Health 2020:1-5. 
Socioeconomic position, modifiable dementia risk and cognitive declice 

Part 2

Public health perspective 

Chapter 4

Dementia awareness and risk

perception in middle-aged

and older individuals: baseline

results of the MijnBreincoach

survey on the association

between lifestyle and brain

health

Irene Heger, Kay Deckers, Martin van Boxtel, Marjolein de Vugt, KlaasJan

Hajema, Frans Verhey, Sebastian Köhler

BMC Public Health, 2019 



\section{Abstract}

Background: The total number of people with dementia is increasing worldwide, due to our aging society. Without a disease-modifying drug available, risk reduction strategies are to date the only promising way to reduce dementia incidence in the future. Substantial evidence exists that lifestyle factors contribute to the risk of dementia, such as physical exercise, mental activity and (non-)smoking. Still, most people seem unaware of a relationship between lifestyle and brain health. This paper investigates dementia literacy and knowledge of modifiable risk and protective factors of dementia in a Dutch population-based sample.

Methods: An online-survey was carried out among 590 community-dwelling people between 40 and 75 years old in the Province of Limburg, the Netherlands. The total group comprises both of a provincial sample $(n=381)$ and a sample of three specific districts within the province $(n=209)$. Dementia awareness and knowledge about 12 risk and protective factors was assessed with items derived from the British Social Attitudes (BSA) survey, supplemented with custom items developed by the research team.

Results: The majority of participants (56\%) were unaware of a relationship between lifestyle and dementia risk. Most individuals identified low cognitive activity, physical inactivity and unhealthy diet as dementia risk factors. Particular gaps in knowledge existed with regard to major cardiovascular risk factors such as hypertension, hypercholesterolemia and coronary heart disease. Although the level of awareness varied by age and level of education, most people $(70 \%)$ were eager to learn more about the topic of brain health, and indicated to be interested in using eHealth (54\%) to measure or improve brain health.

Conclusions: Most people still are unaware of the relation between lifestyle and brain health, indicating the need for public health campaigns. Increasing awareness in the general population about the presence of modifiable dementia risk and protective factors is a crucial first step prior to implementation of preventative measures. Targeting specific subgroups, such as individuals with low socio-economic status and low health literacy, is essential for the reach and effect of a prevention campaign. Outcome of this study was the rationale for an awareness campaign in the Netherlands, called "MijnBreincoach" ("MyBraincoach"). 
Trial registration: Ethics Review Committee Psychology and Neuroscience (ERCPN); reference number 177_07_03_2017

Keywords: dementia literacy, awareness, lifestyle, risk factors, prevention, brain health, health promotion 


\section{Background}

Dementia is a syndrome characterized by cognitive dysfunction leading to interference with daily life activities. Alzheimer's disease and cerebrovascular damage are the most common underlying causes of dementia, with many patients showing evidence for both ${ }^{1,2}$. Dementia is one of the most common causes of disability and mortality among older individuals and has considerable psychosocial effects for both the person with the diagnosis and for relatives and informal caregivers ${ }^{3-5}$. According to the current estimates, 47 million people are living with dementia worldwide. It is expected that this number will triple to 131 million by 2050, with the largest relative increase in low-and middle-income countries ${ }^{6}$. In the Netherlands, 270,000 people had one form of dementia in 2017, and this figure is expected to more than double by $2055^{7}$. The associated global societal economic costs are predicted to rise steadily, making dementia a trillion-dollar disease in $2018^{6,8}$.

Despite extensive global research, there is to date no curative treatment for the common forms of dementia ${ }^{9}$, but several risk factors have been identified. Besides non-modifiable risk factors, such as age, sex and genetics, there is good support for modifiable risk factors as contributors to the risk of developing dementia in later life ${ }^{10}$. Recent estimations suggest that one in three dementia cases may be attributable to common modifiable risk factors ${ }^{11}$. Several healthy living behaviours have been identified, e.g., regular physical exercise, high mental activity and adequate blood pressure control ${ }^{12}$. Development of effective risk reduction strategies to prevent dementia, or delay its onset, is recently receiving increasing attention in research and policy $4,{ }^{13-17}$. With regard to the question when to start targeting these risk and protective factors, it seems that the earlier in life, the better. Research shows that the predictive value of these factors for cognitive impairment and dementia in the very old $(85+)$ is poor ${ }^{18}$. Interventions aimed at promoting healthy lifestyle might therefore be most effective in younger stages of life, such as midlife ${ }^{18-21}$.

Yet, there seems to be a relative lack of dementia risk awareness in the general public, resulting in major gaps of knowledge on dementia in general, and on the relation between lifestyle and brain health in particular. A recent systematic review showed that almost $50 \%$ of all respondents perceive dementia as an inevitable and non-preventable part of living ${ }^{22}$. An Australian survey from 2009 showed that about one-third of all respondents believed that nothing can be done about the risk of dementia, and respondents most often could not 
identify common risk factors ${ }^{23}$. The recent British Social Attitudes (BSA) survey showed that dementia is a major public health concern for most people, but their knowledge of dementia risk factors was poor. Only $1 \%$ of the respondents identified the seven risk and protective factors mentioned in the survey correctly and $22 \%$ could not identify any of the factors. In addition, more than half of participants agreed with the statement "there is nothing one can do to lower one's dementia risk" or said they do not know².

In order to identify specific target groups and address their needs and wishes in future strategies for dementia prevention, the aim of this study was to evaluate dementia literacy and knowledge of dementia risk and protective factors in a well-defined geographical region: the province of Limburg in the South of the Netherlands. We report on the findings of two different samples, as well as differences between certain subgroups (e.g., gender, age, level of education) with regard to dementia risk awareness. Findings were the rationale of an awareness campaign about the relationship between lifestyle and brain health in the province of Limburg, called "MijnBreincoach" ("MyBraincoach"). 


\section{Methods}

\section{Study design and recruitment}

This cross-sectional study is part of MijnBreincoach, a public health campaign of the Alzheimer Centrum Limburg, which is part of the Maastricht University Medical Centre (MUMC+) in the Netherlands. The present study describes the baseline assessment of the public need and pre-campaign level of awareness. The target population for this study were community-dwelling people in midlife (between 40 and 75 years). The study sample was determined in two steps. First, people living in the Province of Limburg who had participated in a previous national health survey (Gezondheidsmonitor 2016) from the municipal health services (GGD) and who agreed to be contacted for future studies were invited to participate (hereafter: the 'provincial sample'). From this total sample, a random number of 711 individuals aged 40-75 years old, stratified by region (North and South Limburg), were invited by email to participate. Because MijnBreincoach consists of both a mass media approach, aimed at the total Province, as well as a community-participation approach to prevention, a random sample of 629 individuals within the relevant age range from three "living labs" in the towns of Brunssum, Landgraaf and Roermond were invited to participate in a second step (hereafter: the 'district sample'). For this, a random selection based on ZIP codes and age (40-75 years) was drawn by the GGD (South Limburg) or the municipality (North Limburg) from the municipal register of the three districts. The three districts were chosen to allow for variation in average neighbourhood socio-economic status. The Ethics Review Committee Psychology and Neuroscience (ERCPN) of Maastricht University approved this study (reference number 177_07_03_2017).

\section{Measurements}

All participants received an invitational e-mail (provincial sample) or letter (district sample) with a unique login code to complete an online informed consent form followed by the actual questionnaire using Qualtrics survey software. The socio-demographic variables gender, age, marital status and level of education were included in the questionnaire. Level of education was obtained by self-assessment of the highest finalized degree and categorized into low (primary school or low vocational education), middle (intermediate secondary education or intermediate vocational or higher secondary education) and high (higher vocational education or university). To assess general dementia literacy, we used ten translated items from the BSA survey 
of the $\mathrm{UK}^{2}$. These items concerned self-reported knowledge of dementia, personal experience with people with dementia, dementia risk awareness and knowledge of five modifiable dementia risk and protective factors (hypertension, smoking, physical activity, depression and diabetes mellitus). We included seven additional modifiable risk and protective factors (obesity, coronary heart disease, chronic kidney disease, hypocholesteraemia, mental activity, low to moderate alcohol intake and healthy diet), in order to assess all the 12 modifiable risk and protective factors included in the "LIfestyle for BRAin Health" (LIBRA) score ${ }^{21,24,25}$. In addition, four sham factors were included (use of painkillers, exposure to ambient noise, personal hygiene and having children) to check for monotone answering tendency. Additional items were developed to evaluate the needs, wishes and barriers of participants concerning brain health, such as the need for further information, preferred information source, subjective barriers to engage in a brain-healthy lifestyle, and motivation to use an internet application to increase risk factor awareness. The total questionnaire consisted of 31 items, with two additional follow-up items for participants who stated to be interested in using an e-Health platform concerning brain health. Most items were set up as statements. Participants were asked to what extent they agreed or disagreed on a 5-point Likert scale ranging from 'strongly agree' to 'strongly disagree'. An English translation of the complete survey is appended in an additional file (Additional File 1).

\section{Statistical analysis}

$\chi 2$ tests were used to examine whether the demographic variables age group ( $<65$ years and $\geq 65$ years), sex, marital status and educational level were associated with level of awareness, knowledge of risk and protective factors and needs, wishes and barriers. All analyses were done in Stata 13.1 (StataCorp, College Station, TX, USA), and the level of statistical significance used was $\mathrm{p}$ $<0.05$ in two-tailed tests. 


\section{Results}

\section{Demographics}

A flowchart of the recruitment process has been added for both the provincial and the district sample as two additional files (Additional File 2 and 3). For the provincial sample, 381 (53.6\%) of the 711 invited individuals participated, and $209(33.2 \%)$ out of 629 for the district sample. In the latter, the response rate of Brunssum was lower (25.7\%) than of Landgraaf (35.7\%) and Roermond (37.1\%). The characteristics of the provincial and district sample are presented in Table 1, and the characteristics of the three districts are summarized in Table 2. Some sample differences in demographic variables were observed. Compared to the provincial sample, participants of the three districts had a significantly lower level of education $\left(\chi^{2}(2)=29.57, p=<.001\right)$. When analysing the three districts separately, it appeared that participants of the district of Brunssum were older, with a higher proportion of people aged 65 years and older $\left(\chi^{2}\right.$ $(2)=18.03, p=<.001$ ), and had significantly lower levels of education than participants of Roermond $\left(\chi^{2}(1)=4.17, p=.041\right)$ and Landgraaf $\left(\chi^{2}(1)=\right.$ $4.17 p=.041)$. 
Table 1. Characteristics of the provincial sample and the district sample.

\begin{tabular}{|c|c|c|}
\hline Sample characteristics & Province of Limburg $(\mathrm{N}=381)$ & Districts $(N=209)$ \\
\hline Age, mean (SD) & $61.1(8.9)$ & $60.1(8.6)$ \\
\hline \multicolumn{3}{|l|}{ Age group (year), $n(\%)$} \\
\hline $40-50$ & $52(13.7 \%)$ & $33(15.9 \%)$ \\
\hline $51-60$ & $115(30.2 \%)$ & $72(34.6 \%)$ \\
\hline $61-70$ & $155(40.7 \%)$ & $78(37.5 \%)$ \\
\hline $71-75$ & $59(15.5 \%)$ & $25(12 \%)$ \\
\hline Female gender, $\mathrm{n}(\%)$ & $164(44 \%)$ & $105(50 \%)$ \\
\hline \multicolumn{3}{|l|}{ Marital status, n (\%) } \\
\hline $\begin{array}{l}\text { Married or living } \\
\text { together }\end{array}$ & $299(79.5 \%)$ & $172(82.3 \%)$ \\
\hline Not or never been married & $18(4.8 \%)$ & $12(5.7 \%)$ \\
\hline Divorced & $33(8.8 \%)$ & $15(7.2 \%)$ \\
\hline Widowed & $26(6.9 \%)$ & $10(4.8 \%)$ \\
\hline \multicolumn{3}{|l|}{ Educational level $^{1}, \mathrm{n}(\%)$} \\
\hline Low & $46(12.2 \%)$ & $55(26.3 \%)^{* * * *}$ \\
\hline Middle & $134(35.6 \%)$ & $88(42.1 \%)$ \\
\hline High & 196 (52.1\%) & 66 (31.6\%) \\
\hline
\end{tabular}

Note: ${ }^{1}$ Level of education was self-reported and categorized as follows: low (primary school or low vocational education), middle (intermediate secondary education or intermediate vocational or higher secondary education) and high (higher vocational education or university). ${ }^{* * *} p<0.001$. 


\section{Table 2. Characteristics of the three districts.}

\begin{tabular}{|c|c|c|c|}
\hline Sample characteristics & Roermond $(\mathrm{N}=78)$ & Landgraaf $(\mathrm{N}=75)$ & Brunssum $(\mathrm{N}=56)$ \\
\hline Age, mean (SD) & $56.9(8.7)$ & $60.9(7.7)$ & $63.3(8.1) * * *$ \\
\hline \multicolumn{4}{|l|}{ Age group (year), $n(\%)$} \\
\hline $40-50$ & $19(24.4 \%)$ & $8(10.8 \%)$ & $6(10.7 \%)$ \\
\hline $51-60$ & $31(39.7 \%)$ & $26(35.1 \%)$ & $15(26.8 \%)$ \\
\hline $61-70$ & $24(30.8 \%)$ & $31(41.9 \%)$ & $23(41.1 \%)$ \\
\hline $71-75$ & $4(5.1 \%)$ & $9(12.2 \%)$ & $12(21.4 \%)$ \\
\hline Female gender, n (\%) & $39(50 \%)$ & $38(51 \%)$ & $28(50 \%)$ \\
\hline \multicolumn{4}{|l|}{ Marital status, n (\%) } \\
\hline Married or living together & $66(85 \%)$ & $64(85 \%)$ & $42(75 \%)$ \\
\hline Not or never been married & $4(5 \%)$ & $4(5 \%)$ & $4(7 \%)$ \\
\hline Divorced & $5(6 \%)$ & $5(7 \%)$ & $5(9 \%)$ \\
\hline Widowed & $3(4 \%)$ & $2(3 \%)$ & $5(9 \%)$ \\
\hline \multicolumn{4}{|l|}{ Educational level ${ }^{1}, \mathrm{n}(\%)$} \\
\hline Low & $18(23 \%)$ & $15(20 \%)$ & $22(39 \%) *$ \\
\hline Middle & $32(41 \%)$ & $33(44 \%)$ & $23(41 \%)$ \\
\hline High & $28(36 \%)$ & $27(36 \%)$ & $11(20 \%)$ \\
\hline
\end{tabular}

Note: ${ }^{1}$ Level of education was self-reported and categorized as follows: low (primary school or low vocational education), middle (intermediate secondary education or intermediate vocational or higher secondary education) and high (higher vocational education or university). *** $p<0.001$, $* \mathrm{p}<0.05$. 


\section{Dementia literacy}

Of the total sample, $44 \%$ of the respondents $(n=254)$ were aware of a relationship between brain health and lifestyle by stating that dementia risk reduction is possible. People with a lower level of education $\left(\chi^{2}(2)=53.46\right.$, $p<.001)$ and people aged 65 years and older $\left(\chi^{2}(1)=9.12, p<.01\right)$ were less likely to agree that dementia risk reduction is possible. No differences were found between men and women $\left(\chi^{2}(1)=0.42, p=.517\right)$, the provincial sample and the district sample $\left(\chi^{2}(1)=2.78, p=.10\right)$, and between districts $\left(\chi^{2}(2)=1.47, p=.480\right)$.

\section{Knowledge on dementia risk and protective factors}

More than half of the total sample (59\%) identified zero to four of the twelve factors, with more than $10 \%$ unable to recognize any of them and only $1.7 \%$ identifying all factors correctly. Figure 1 presents the percentage of identified dementia risk and protective factors for the provincial sample and the district sample. Figure 2 displays a comparison between the three districts. Figures 3 and 4 give an overview of the amount of correctly identified risk and protective factors in both the provincial and district sample and for the three districts separately. In both the provincial and the district sample, a cognitively active lifestyle was identified most often (province 80\%; districts 79\%), followed by physical activity (province 66\%; districts 59\%) and a healthy diet (province $52 \%$; districts 47\%). Vascular factors such as hypertension (province 30\%; districts 23\%\%), hypercholesterolemia (province 27\%; districts 25\%), coronary heart disease (province 17\%; districts 14\%) and chronic kidney disease (province 11\%; districts 8\%) were identified least often. With regard to the sham factors (use of painkillers, exposure to ambient noise, personal hygiene and having children), more than $90 \%$ of the participants correctly rejected them as valid risk factors for dementia. Significantly fewer risk and protective factors were identified by participants with lower education $\left(\chi^{2}(12)\right.$ $=35.94, p=<.001)$, except for the risk factors depression $\left(\chi^{2}(1)=2.46\right.$, $p=.117)$, hypercholesterolemia $\left(\chi^{2}(1)=1.79, p=.181\right)$ and coronary heart disease $\left(\chi^{2}(1)=3.46, p=.063\right)$. Participants who stated that their level of knowledge concerning dementia was excellent, good or considerable identified significantly more risk and protective factors than participants stating that their knowledge was poor $\left(\chi^{2}(12)=28.50, p=<.01\right)$. 


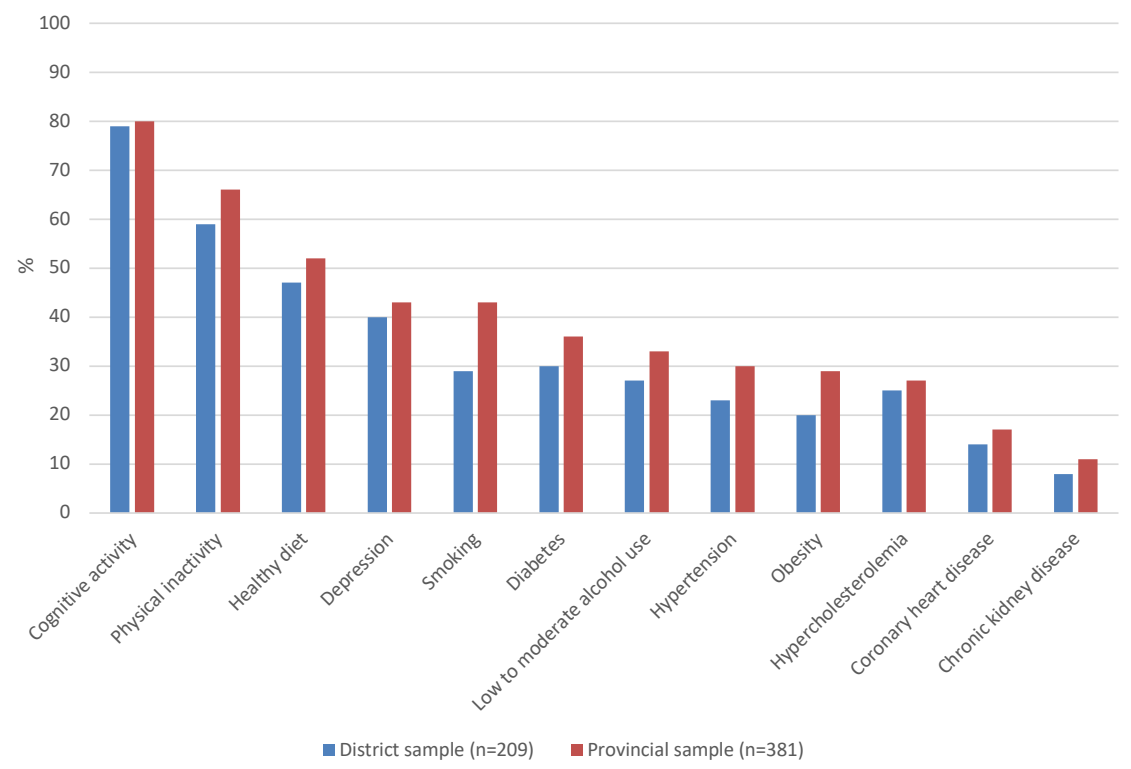

Figure 1. Identified risk and protective factors for the provincial sample and the district sample.

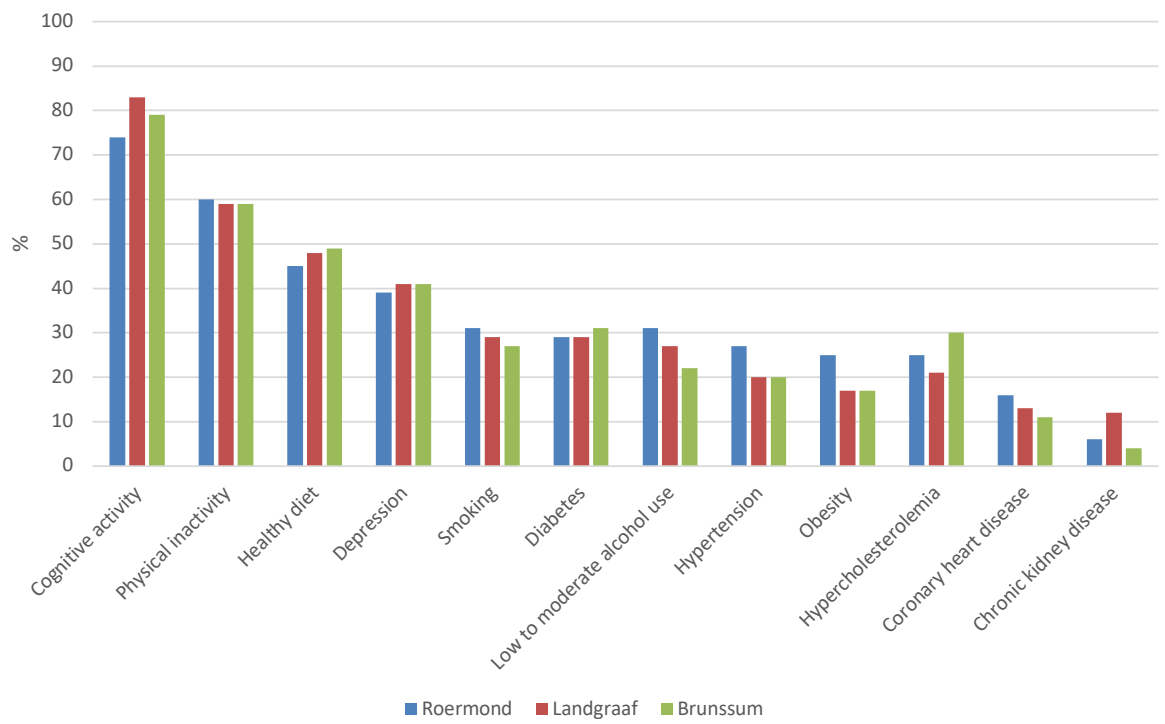

Figure 2. Identified risk and protective factors for the three districts. 


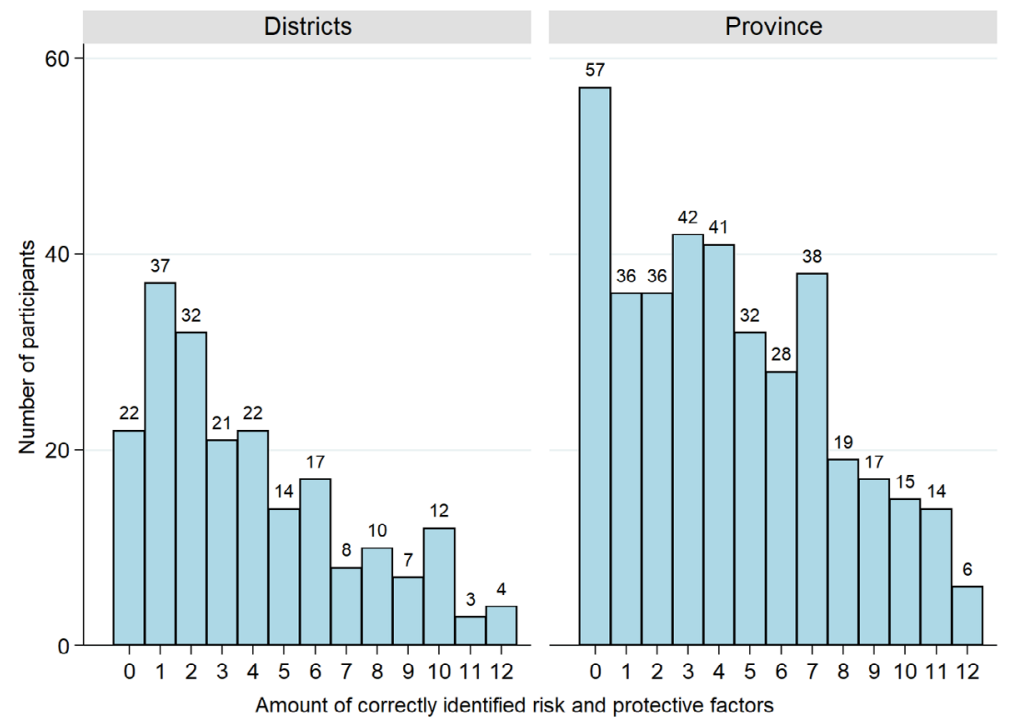

Figure 3. Amount of correctly identified risk and protective factors for the provincial sample and the district.
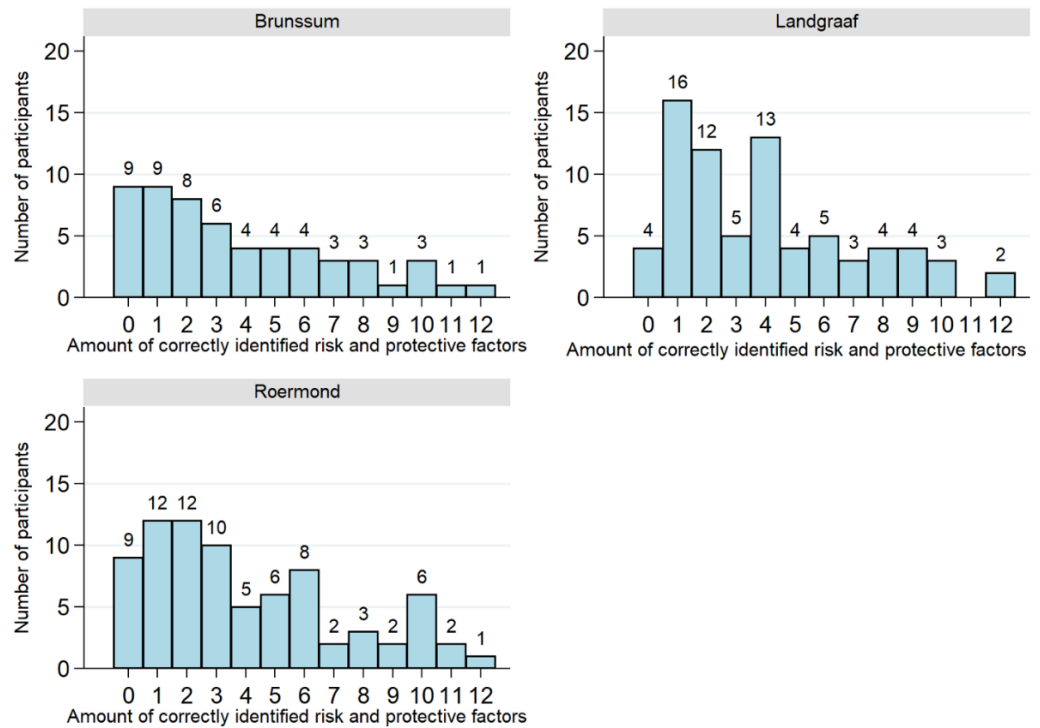

Figure 4. Amount of correctly identified risk and protective factors for the three districts separately. 
Needs, wishes and barriers

The majority of participants $(n=382,70 \%)$ stated they would like to receive more information about the relationship between lifestyle and brain health. Most people preferred information by searching on the web (province 61\%; districts 56\%), followed by visiting the website of the Dutch Alzheimer's Association (province 36\%; districts 39\%), consulting their general practitioner (province 36\%; districts 43\%), visiting the website of the municipal health services (province 14\%; districts 19\%) and visiting the library (province 5\%; districts $3 \%)$. People from the provincial sample $\left(\chi^{2}(2)=17.27, \mathrm{p}=<.001\right)$ and people with a higher level of education $\left(\chi^{2}(4)=11.96, p<.05\right)$ were more likely to request further information. Fifty-four percent $(n=291)$ of the participants stated they would like to use an Internet application in order to learn more about improving their brain health, with another $27 \%(n=147)$ willing to consider it. No statistical differences with regard to the use of an Internet application were observed between the provincial and district sample $\left(\chi^{2}(2)=0.06, p=0.971\right)$ nor for level of education $\left(\chi^{2}(4)=4.93, p=0.294\right)$ and sex $\left(\chi^{2}(2)=5.65, p=0.06\right)$. The majority of participants aged 65 years old or above would use, or consider using, an Internet application $(n=176,81 \%)$, which was not significantly different $\left(\chi^{2}(2)=2.92, p=.232\right)$ from younger ages $(n=266,81 \%)$. The largest barrier for adopting a brain-healthy lifestyle was stated to be lack of knowledge (province 42\%; districts 38\%), followed by lack of motivation (province 17\%; districts 13\%) and lack of time (province 14\%; districts 11\%). Other barriers were difficulty organizing (province 8\%, districts 5\%), financial reasons (province 4\%; districts 6\%), health problems (province 4\%; districts 2\%) and 'other reasons' (province 4\%, districts 3\%). 


\section{Discussion}

This study assessed dementia literacy and knowledge concerning dementia risk and protective factors in middle-aged and older individuals living in the community. Results clearly showed that the majority of individuals were unaware of the relationship between lifestyle-related risk and protective factors and brain health. Considerable gaps in knowledge exist regarding common dementia risk factors such as hypertension, hypercholesterolemia and coronary heart disease. Most people were eager to receive more information on the topic of brain health and most participants were also positive about using eHealth tools to improve brain health. Variation in level of awareness by level of education and age was observed.

The results of this study are in line with previous studies reporting on the level of awareness of the relationship between lifestyle and brain health and risk and protective factors for dementia ${ }^{2,22,23}$. The methodology of this study is comparable to the BSA study in the UK, and therefore it is noteworthy that the UK and Dutch population show very similar levels of awareness, with less than half of the participants (47\% and 44\%, respectively) reporting that the risk for dementia can be influenced ${ }^{2}$. As for the identification of lifestyle factors, our study is also congruent with the findings of a recent systematic review examining population surveys concerning the public's knowledge and understanding of dementia ${ }^{22}$. Cognitive activity was identified most often as a protective factor ${ }^{22}$. Despite the good evidence for cardiovascular risk factors such as hypertension, hypercholesterolemia and diabetes ${ }^{12,15,26,27}$, only few people seem aware that "what is good for your heart is good for your brain".

Together, these studies make a strong case for informing the public more effectively about modifiable dementia risk and protective factors. The report of the National Academies of Sciences, Engineering and Medicine (NASEM) from 2017 states that evidence for risk and protective factors of brain health is still inconclusive, yet compelling. The report stresses the importance of providing the public with easily accessible information about the effect of targeting those risk factors that are supported by promising research, i.e., cognitive activity, physical activity and adequate blood pressure control ${ }^{12}$. The Lancet Commission on Dementia Prevention, Intervention and Care underlines this by recommending 'to be ambitious about dementia prevention'. The Commission underscores that society as a whole has a responsibility to not 
only provide information about dementia prevention, but also implement low-level interventions ${ }^{4}$.

It seems that people with a lower socioeconomic status and older people are more difficult to reach and motivate in eHealth and preventative campaigns ${ }^{28-32}$. This was also seen in our study, in which the subgroups with significantly lower dementia literacy (i.e., people with lower levels of education) were less inclined to receive further information. A one-size-fits-all approach might therefore not be efficient with regard to prevention strategies. Indeed, recent literature showed that targeting specific subgroups and engagement of local partners is crucial for the reach and effect of a prevention campaign ${ }^{32}$.

The current findings serve as a baseline for a dementia awareness campaign that has been launched recently in the Province of Limburg, the Netherlands, called MijnBreincoach ('MyBraincoach'33. As part of this campaign, an eHealth platform has been developed aimed at giving people insight into their own lifestyle profile and pointing them towards individual 'room for improvement'. To investigate differences in preventive strategies, the approach is two folded. On the one hand, it targets the whole Province of Limburg through mass media (e.g., interviews in newspapers and TV), posters and flyers in public spaces and involvement of cultural and health-related authorities. On the other hand, a district-oriented campaign implements measures tailored towards the needs and wishes of the three districts that were part of our survey. The latter approach was developed together with the local community, local professionals and health care providers, offering a variety of activities (e.g., lectures in community centres, training to local health care providers, involvement of small and medium-sized enterprises).

To our knowledge, this is the first study of dementia literacy in a Dutch population and one of the first studies worldwide to combine the results of a dementia literacy assessment with an awareness campaign fitted to the same target population. Strengths of our study include the use of a comparable methodology as previous dementia literacy studies ${ }^{2}$ in order to add up to the existing evidence. Our study has, however, some limitations. First, selection bias may have occurred in general since non-Dutch speakers were excluded, which likely excludes minority groups who are less educated and health literate. Second, the potential for the use of Internet applications found in our study could be an overestimation since our study design consisted mainly of online 
questionnaires. Third, it should be taken in to consideration that those aged 65 years and older, who were less aware of dementia risk reduction, related this question to themselves and therefore answered differently than when asking about 'risk reduction in general'. Unfortunately, we do not have any data on why people do not agree that 'dementia risk reduction is possible'. This would be an interesting question to add in future research. However, we have shown in previous studies that the LIBRA-index still predicts cognitive deterioration and incident dementia in this age group ${ }^{18,20,21}$. So from our perspective, they belong to the same target group as people aged younger than 65 years old disagreeing with the statement that dementia risk reduction is possible. Furthermore, the provincial sample was composed of participants from the Gezondheidsmonitor 2016 who agreed to participate in further research. This pre-selection of people willing to participate in scientific research might not be an exact reflection of the general population, due to factors such as level of education and general health knowledge, which might lead to an overestimation of awareness levels. In addition, we could not compare the demographics of those who did and did not take part in this study because linkage of the two surveys (datasets) was only possible in those that did consent to participate in the present study. Still, our findings are comparable to the results of the BSA study in the UK. Also, differences between the provincial and district samples were minimal. Regarding the latter, the Brunssum district showed the lowest level of awareness and the lowest level of education. It should be noted that this district was selected a priori because of its known, relatively low socioeconomic status.

\section{Conclusions}

Our study corroborates the evidence that the majority of people in the general population are unaware of a relationship between lifestyle-related factors and brain health, and most people in this study expressed a need for brainhealth education. Major gaps in knowledge exist in particular amongst the cardiovascular risk factors for dementia. These findings stress the importance of informing the public about lifestyle related risk and protective factors of brain health and dementia via health promotion campaigns. 


\section{References}

1. O'Donnell CA, Browne S, Pierce M, et al. Reducing dementia risk by targeting modifiable risk factors in mid-life: study protocol for the Innovative Midlife Intervention for Dementia Deterrence (InMINDD) randomised controlled feasibility trial. Pilot Feasibility Stud 2015;1:40.

2. Marcinkiewicz A, Reid S, Attitudes to dementia: Findings from the 2016 British Social Attitudes survey 2016, NatCen Social Research: London.

3. Ritchie K, Lovestone S. The dementias. Lancet 2002;360:1759-1766.

4. Livingston G, Sommerlad A, Orgeta V, et al. Dementia prevention, intervention, and care. The Lancet 2017;390:26732734.

5. Berr C, Wancata J, Ritchie K. Prevalence of dementia in the elderly in Europe. Eur Neuropsychopharmacol;15:463-471.

6. Prince M, Comas A, Knapp M, Guerchet MK, M., World Alzheimer Report 2016: improving healthcare for people living with dementia. 2016, Alzheimer's Disease International: London.

7. Feiten over dementie. 2017; Beschikbaar op: https://www.alzheimer-nederland. nl/dementie/feiten-cijfers. Geraadpleegd op 19 Jan.

8. Michalowsky B, Thyrian JR, Eichler T, et al. Economic Analysis of Formal Care, Informal Care, and Productivity Losses in Primary Care Patients who Screened Positive for Dementia in Germany. $J$ Alzheimers Dis 2016;50:47-59.

9. Rogers NT, Steptoe A, Cadar D. Frailty is an independent predictor of incident dementia: Evidence from the English Longitudinal Study of Ageing. Sci Rep 2017;7:1-7.

10. O'Donnell CA, Manera V, Kohler S, Irving K. Promoting modifiable risk factors for dementia: is there a role for general practice? Br J Gen Pract 2015;65:567568.

11. Norton S, Matthews FE, Barnes DE, Yaffe K, Brayne C. Potential for primary prevention of Alzheimer's disease: an analysis of population-based data. Lancet Neurol 2014;13:788-794.

12. Downey A, Stroud C, Landis S, Leshner AI, Preventing Cognitive Decline and Dementia: A Way Forward. 2017, The National Academies Press: Washington (DC). p. 1-161

13. Anstey KJ, Peters $\mathrm{R}$, Clare L, et al. Joining forces to prevent dementia: The International Research Network On Dementia Prevention (IRNDP). Int Psychogeriatr 2017;29:1757-1760.

14. Fratiglioni L, Qiu C. Prevention of cognitive decline in ageing: dementia as the target, delayed onset as the goal. Lancet Neurol. 2011;10:778-779.

15. Gorelick PB, Furie KL, Iadecola $C$, et al. Defining Optimal Brain Health in Adults: A Presidential Advisory From the American Heart Association/American Stroke Association. Stroke 2017;48.

16. Lincoln P, Fenton $K$, Alessi $C$, et al. The Blackfriars Consensus on brain health and dementia. Lancet 2014;383:1805-1806. 
17. Lee ATC, Richards M, Chan WC, Chiu HFK, Lee RSY, Lam LCW. Association of Daily Intellectual Activities With Lower Risk of Incident Dementia Among Older Chinese Adults. JAMA Psychiatry 2018.

18. Deckers K, Kohler S, van Boxtel M, Verhey F, Brayne C, Fleming J. Lack of associations between modifiable risk factors and dementia in the very old: findings from the Cambridge City over-75s cohort study. Aging Ment Health 2017;22:1272-1278.

19. Winblad B, Amouyel P, Andrieu S, et al. Defeating Alzheimer's disease and other dementias: a priority for European science and society. Lancet Neurol 2016;15:455532.

20. Schiepers OJG, Kohler S, Deckers K, et al. Lifestyle for Brain Health (LIBRA): a new model for dementia prevention. Int $J$ Geriatr Psychiatry 2018;33:167-175.

21. Vos SJB, van Boxtel MPJ, Schiepers OJG, et al. Modifiable Risk Factors for Prevention of Dementia in Midlife, Late Life and the Oldest-Old: Validation of the LIBRA Index. $J$ Alzheimers Dis 2017;58:537-547.

22. Cations M, Radisic G, Crotty M, Laver KE. What does the general public understand about prevention and treatment of dementia? A systematic review of population-based surveys. PLoS One 2018;13.

23. Low LF, Anstey KJ. Dementia literacy: recognition and beliefs on dementia of the Australian public. Alzheimers Dement 2009;5:43-49.

24. Deckers K, van Boxtel MP, Schiepers OJ, et al. Target risk factors for dementia prevention: a systematic review and Delphi consensus study on the evidence from observational studies. Int $J$ Geriatr Psychiatry 2015;30:234-246.

25. Pons A, LaMonica HM, Mowszowski L, Kohler S, Deckers K, Naismith SL. Utility of the LIBRA Index in Relation to Cognitive Functioning in a Clinical Health Seeking Sample. J Alzheimers Dis 2018;62:373-384

26. Deckers K, Schievink SHJ, Rodriquez MMF, et al. Coronary heart disease and risk for cognitive impairment or dementia: Systematic review and metaanalysis. PLoS One 2017;12.

27. Schievink SHJ, van Boxtel MPJ, Deckers $\mathrm{K}$, van Oostenbrugge RJ, Verhey FRJ, Kohler S. Cognitive changes in prevalent and incident cardiovascular disease: a 12 year follow-up in the Maastricht Aging Study (MAAS). Eur Heart J 2017.

28. de Veer AJ, Peeters JM, Brabers AE, Schellevis FG, Rademakers JJ, Francke AL. Determinants of the intention to use e-Health by community dwelling older people. BMC Health Serv Res 2015; 15:103.

29. LaMonica HM, English A, Hickie IB, et al. Examining Internet and eHealth Practices and Preferences: Survey Study of Australian Older Adults With Subjective Memory Complaints, Mild Cognitive Impairment, or Dementia. $J$ Med Internet Res 2017;19.

30. Eakin EG, Bull SS, Glasgow RE, Mason M. Reaching those most in need: a review of diabetes self-management interventions in disadvantaged populations. Diabetes 
Metab Res Rev 2002;18:26-35.

31. Cleland V, Ball K. Recruiting hard-toreach populations: lessons from a study of women living in socioeconomically disadvantaged areas of Victoria, Australia. Health Promot J Austr 2010;21:243-244.

32. Luten KA, Dijkstra A, de Winter AF, Reijneveld SA. Developing a communitybased intervention for Dutch older adults in a socioeconomically disadvantaged community. Health Promot Int 2018.

33. We zijn zelf het medicijn. Beschikbaar op: https://www.wezijnzelfhetmedicijn.nl/. Geraadpleegd op 31-08-2018. 


\section{Additional Files}

\section{Additional File 1. English language translation of the survey}

\section{Demographics}

1. How old are you?

[textfield for number between 40 and 75]

2. What is your gender?

$\begin{array}{ll}\text { o } & \text { Male } \\ \text { o } & \text { Female }\end{array}$

3. What is your zip code?

[textfield of four numbers]

4. What is your marital status?

o Married/registered partnership

o Living together

o Unmarried, never been married

o Divorced

o Widowed

5. What is your highest finalized degree of education?

[Six categories according to the Dutch education system, categorized into low, medium and high]

\section{Dementia knowledge}

1. Dementia describes a set of symptoms including loss of memory, sudden mood swings, not remembering who people are, and having trouble finding your words. Alzheimer's disease is one form of dementia. How much would you say you know about dementia?
o A great deal
o Quite a lot
o Some
o Not very much 
$\begin{array}{ll}\text { o } & \text { Nothing at all } \\ \text { o } & \text { I don't know } \\ \text { o } & \text { I prefer not to answer this question }\end{array}$

2. Have you ever personally known anyone with dementia or have it yourself? [Please choose all that apply]

- $\quad$ No, I don't know anyone who has, or had, dementia

- $\quad$ Yes, my job involves / involved working with people who have dementia

- $\quad$ Yes, I have dementia myself

- $\quad$ Yes, my partner, parents (in-law), or children

- $\quad$ Yes, my grandparents or brother/sister

- $\quad$ Yes, a friend(s) I know fairly well

- $\quad$ Yes, a friend(s) or acquaintance(s) I know less well

- $\quad$ Yes, a colleague / someone at my work

- $\quad$ Yes, someone else

- I am not sure

- $\quad$ I prefer not to answer this question

3. Leaving aside anything you have done as part of your job, please say which, if any, of the following you have done for someone with dementia, either now or in the past?

[Please choose all that apply]

- $\quad$ Cared for someone (other than myself) who lives / lived with me

- $\quad$ Cared on a regular basis for someone who does not / did not live with me

- $\quad$ Been responsible for making arrangements for someone to be looked after (e.g., arranged for a carer to visit someone at home, or arranged for someone to go into a residential home)

- Helped someone from time-to-time

- $\quad$ Encouraged someone showing symptoms of dementia to seek professional help

- $\quad$ Visited someone every now and then

- $\quad$ Other [textfield]

- $\quad$ None of these

- I prefer not to answer this question 


\section{Dementia risk awareness}

Please state how much you agree or disagree with the following statements.

4. 'There is nothing anyone can do to reduce their risks of getting dementia'

o Agree strongly

o Agree

o Neither agree nor disagree

o Disagree

o Disagree strongly

5. 'High blood pressure increases your chances of getting dementia'

o Agree strongly

o Agree

o Neither agree nor disagree

o Disagree

o Disagree strongly

6. 'Having a parent with dementia increases the chances of getting dementia'

o Agree strongly

o Agree

o Neither agree nor disagree

o Disagree

o Disagree strongly

7. 'The use of painkillers increases your chances of getting dementia'

o Agree strongly

o Agree

o Neither agree nor disagree

o Disagree

o Disagree strongly

8. 'Smoking increases your chances of getting dementia'

o Agree strongly

o Agree

o Neither agree nor disagree

o Disagree

o Disagree strongly 
9. 'No or moderate alcohol use lowers your chances of getting dementia'

o Agree strongly

o Agree

o Neither agree nor disagree

o Disagree

o $\quad$ Disagree strongly

10. 'Regular physical activity lowers your chances of getting dementia'

o Agree strongly

o Agree

o $\quad$ Neither agree nor disagree

o Disagree

o Disagree strongly

11. 'Working in a noisy environment increases your chances of getting dementia'

o Agree strongly

o Agree

o Neither agree nor disagree

o Disagree

o Disagree strongly

12. 'Depression increases the chances of getting dementia'

o Agree strongly

o Agree

o Neither agree nor disagree

o Disagree

o Disagree strongly

13. 'Diabetes increases the chances of getting dementia'
o Agree strongly
o Agree
o $\quad$ Neither agree nor disagree
o Disagree
o Disagree strongly

14. 'Being overweight increases the chances of getting dementia'

o Agree strongly 


$\begin{array}{ll}\text { o } & \text { Agree } \\ \text { o } & \text { Neither agree nor disagree } \\ \text { o } & \text { Disagree } \\ \text { o } & \text { Disagree strongly }\end{array}$

15. 'Poor personal hygiene increases the chances of getting dementia'

$\begin{array}{ll}\text { o } & \text { Agree strongly } \\ \text { o } & \text { Agree } \\ \text { o } & \text { Neither agree nor disagree } \\ \text { o } & \text { Disagree } \\ \text { o } & \text { Disagree strongly }\end{array}$

16. 'A mentally active lifestyle lowers the chances of getting dementia'
o Agree strongly
o Agree
o Neither agree nor disagree
o Disagree
o Disagree strongly

17. 'Heart disease increases the chances of getting dementia'
o Agree strongly
o Agree
o Neither agree nor disagree
o Disagree
o Disagree strongly

18. 'Kidney disease increases the chances of getting dementia'
o Agree strongly
o Agree
o Neither agree nor disagree
o Disagree
o Disagree strongly

19. 'Having children increases the chances of getting dementia'
o $\quad$ Agree strongly
o Agree
o Neither agree nor disagree
o Disagree 
o Disagree strongly

20. 'High cholesterol increases the chances of getting dementia'

o Agree strongly

o Agree

o Neither agree nor disagree

o Disagree

o Disagree strongly

21. 'Healthy diet lowers the chances of getting dementia'

$\begin{array}{ll}\text { o } & \text { Agree strongly } \\ \text { o } & \text { Agree } \\ \text { o } & \text { Neither agree nor disagree } \\ \text { o } & \text { Disagree } \\ \text { o } & \text { Disagree strongly }\end{array}$

22. Here is a list of factors that influence the risk of getting dementia, together with factors that have no influence on the risk of getting dementia. Which of the factors described below are the most important factors for the development of dementia according to you?

[Three options possible]

o High blood pressure

o Having parents with dementia

o Use of painkillers

o Smoking

o $\quad$ No or moderate use of alcohol

o Regular physical activity

o Working in a noisy environment

o Depression

o Diabetes

o Obesity (being overweight)

o Poor personal hygiene

o A mentally active lifestyle

o Heart disease

o Kidney disease

o Having children

o High cholesterol

o Healthy diet 
23. Would you be interested in receiving information on how to improve your brain health?

$\begin{array}{ll}\text { o } & \text { Yes } \\ \text { o } & \text { No } \\ \text { o } & \text { Maybe }\end{array}$

24. In the case that you would like to know more about your own brain health, what infor-mation channels would you use to find this information?

[Please choose all that apply]

- $\quad$ Search on the web

- $\quad$ GP office

- Webpage municipal health services

- Website Dutch Alzheimer's Association

- Library

- $\quad$ Other [textfield]

- $\quad$ None of these

- I do not know

- $\quad$ I prefer not to answer this question

25. In the case that you would decide to work on improving your own brain health, what could be an obstructing factor for you?

[Please choose all that apply]
o Lack of knowledge
o Lack of time
o Financial reasons
o Lack of motivation
o Difficult to organize
o Health problems
o Other [textfield]
o None of these
o I do not know
o I prefer not to answer this question

26. In the case that there was a mobile application, providing you without charge with in-formation about your brain health and giving advice on how to improve your brain health, would you use this app?

$\begin{array}{ll}\text { o } & \text { Yes } \\ \text { o } & \text { No }\end{array}$


o Maybe

[If answer is No, go to End]

[If answer is Yes or Maybe, go the question 27]

27. Maastricht University has started a research line focused on prevention of dementia, in collaboration with the municipal health services. This research includes the devel-opment of an app (mobile application) that could give you more insight into your own brain health and how to improve your brain health. Would you be interested and do you give consent to be contacted for this research?

o Yes, I give consent

o No, I give no consent

[If answer is No, go to End]

[If answer is Yes, go to question 28]

28. Please leave the phone number and/or e-mail-address that we can use to contact you for this research.

[Text field]

\section{End}

Thank you very much for filling in this questionnaire. If you have any remarks or questions regarding this study, please contact us via [email address]. 
Additional File 2. Flowchart of the recruitment process of the provincial sample

Participants of the Dutch Health

Survey who consented to be

contacted for further studies

$\mathrm{N}=1000$

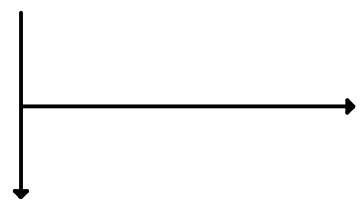

Random exclusion

$$
\mathbf{N}=\mathbf{2 8 9}
$$

Random selection based on

age and region

$\mathrm{N}=711$

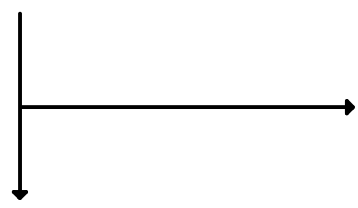

Non-responce

$\mathbf{N}=330$

Participants included

in the survey

$\mathbf{N}=381$ 


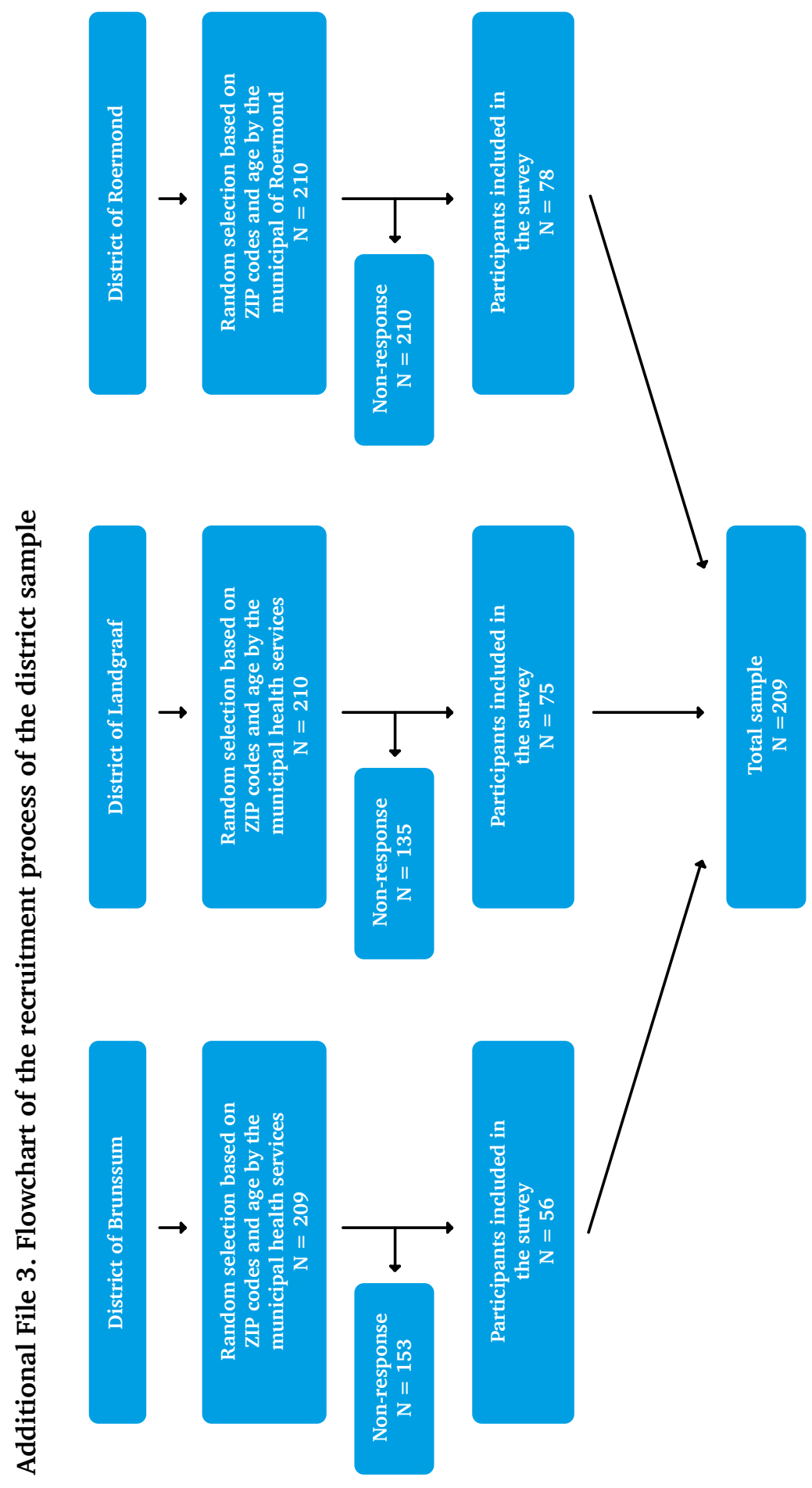



Chapter 5

Raising awareness for dementia risk reduction through a public health campaign: a pre-post study

Irene Heger, Sebastian Köhler, Martin van Boxtel, Marjolein de Vugt, KlaasJan Hajema, Frans Verhey, Kay Deckers

BMJ, 2020 



\section{Abstract}

Objectives: Evaluate the effect of a health promotion campaign aimed at increasing awareness about dementia risk reduction in middle-aged community-dwelling individuals in the Netherlands.

Design: A 10-month public health campaign using mass-media and community participation, supported by eHealth. Population-level difference in awareness before and after the campaign, including variation between demographic groups, was assessed in two independent cross-sectional samples from the same target population.

Setting: The public health campaign was launched in the Province of Limburg, the Netherlands, targeting all inhabitants aged 40 to 75 years old. Three specific districts within the Province were chosen for an additional communityparticipation approach, in which local stakeholders were invited to support the campaign.

Results: No pre- $(n=590)$ post- $(n=602)$ difference was observed in people agreeing to the statement that dementia risk reduction is possible $\left(\mathrm{X}^{2}(1)=\right.$ $1.27, \mathrm{p}=0.260)$. For the individual lifestyle factors, physical activity $(7.6 \%$ increase $\left.\left(\mathrm{X}^{2}(1)=7.48, p=.006\right)\right)$ and healthy diet $\left(10.5 \%\right.$ increase $\left(\mathrm{X}^{2}(1)\right.$ $=12.37, p \leq 0.001)$ ) were identified more often as being protective against dementia post-campaign. Of all risk/protective factors assessed, cognitive activity was identified most often at both pre- $(79.4 \%)$ and post-assessment (80.4\%), but there was no increase in awareness $\left(\mathrm{X}^{2}(1)=0.17, p=0.677\right)$. Self-reported exposure to the campaign was associated with greater awareness and motivation for behavioural change $\left(\mathrm{X}^{2}(1)=6.52, p=.011\right)$. Compared to mass media only, the addition of community-participation resulted in better recognition of campaign-material and the eHealth-platform.

Conclusions: This study was not able to reach a population-level increase of awareness of dementia risk reduction. Two out of the three lifestyle factors that formed the foundation of the campaign were identified more often postcampaign. Those reported having been exposed to the campaign were more aware and more inclined towards behavioural change. 


\section{Introduction}

Dementia is characterized by progressive decline of cognitive abilities, leading to interference in daily living. Alzheimer's disease (AD) and vascular dementia are the most common underlying pathologies, and often co-exist ${ }^{1}$. Due to the aging population, the number of people living with dementia worldwide is expected to triple from 50 million in 2018 to 152 million in $2050^{2}$. This rapid global increase and the absence of a curative treatment exposes a major public health concern.

Primary prevention of dementia through lifestyle modification is gaining increasing attention in research and policy ${ }^{1,3-5}$. Population-based estimations show that around one-third of all dementia cases might be attributable to seven lifestyle and health-related factors, including physical inactivity, depression, and low mental stimulation ${ }^{4}$. Reducing exposure to these risk factors by 10 to $20 \%$ per decade would lower the prevalence of $\mathrm{AD}$ by as much as 8 to $15 \%{ }^{4}$. These insights have led to a series of randomized controlled trials (RCTs), using lifestyle interventions to delay or prevent cognitive decline and dementia onset $^{6-9}$. Neither the multi-domain Prevention of Dementia by Intensive Vascular Care (preDIVA) trial, in 3,454 patients with known vascular risk factors aged 70-79 years, nor the Multidomain Alzheimer Preventive Trial (MAPT) in 1,680 adults aged 70 years and older resulted in significant reduction of incident dementia ${ }^{7}$ and change in memory function ${ }^{8}$, or only in subgroups. In contrast, the population-based Finnish Geriatric Intervention Study to Prevent Cognitive Impairment and Disability (FINGER), starting earlier in those aged 60-77 years, was the first large-scale RCT that showed beneficial effects on cognition $^{6,10}$. It seems that dementia risk reduction interventions should target people in midlife to minimize lifetime accumulation of risk factor exposure and consequent brain pathology $\mathrm{y}^{1,11-13}$

Recently, a global initiative aimed at harmonizing intervention studies on risk factor modification was launched, called World Wide (WW) FINGERS ${ }^{11}$. Incorporated trials include the US Study to Protect Brain Health Through Lifestyle Intervention to Reduce Risk (US POINTER) and the Singapore intervention study to prevent cognitive impairment and disability (SINGER) ${ }^{11}$. The ambition of WW-FINGERS to find robust evidence for lifestyle interventions that delay or prevent dementia onset is promising from a public health perspective. In addition, epidemiological studies have shown decreasing incidence of dementia in high-income countries, observed in several cohorts ${ }^{14-16}$, 
probably because of improved cardiovascular health, nutrition and education over the last decades ${ }^{2}$. The timeliness of dementia prevention interventions was also emphasized by the publication of guidelines for risk reduction of cognitive decline and dementia by the World Health Organization (WHO) in $2019^{5}$ and the 2020-report of the Lancet Commission on Dementia Prevention, Intervention and Care. ${ }^{17}$

It must, however, be noted that the general public is still largely unaware of the potential of dementia risk reduction, let alone of specific actions to reduce dementia risk ${ }^{18-21}$. In our own survey among middle-aged adults $(n=590), 44 \%$ were aware of dementia risk reduction, and only $20-25 \%$ considered vascular conditions to increase the risk. ${ }^{18}$ Hence, despite the need for conclusive RCTs, little is known about how knowledge can be translated to the general public to raise awareness, and how to engage hard-to-reach subgroups (e.g., low health literacy or socioeconomic status) who are often underrepresented in clinical trials, too ${ }^{22-24}$. Creating risk awareness at the population level is a crucial first step before behaviour-change programs can be developed and implemented.

Dementia awareness campaigns have focused on topics as improving recognition of dementia ${ }^{25}$, dementia care $^{26}$, decreasing public stigma ${ }^{27,28}$, and few on dementia risk reduction ${ }^{29,30}$. An Australian study using an informative website on dementia risk reduction resulted in increased knowledge and motivation to engage in relevant health behaviours. However, no populationlevel measurements for evaluation were used and the study only included a post-intervention assessment of people visiting the website ${ }^{29}$. One populationbased national awareness campaign in Ireland found a significant increase in people agreeing that "there are things you can do to reduce your risk". However, awareness of dementia risk reduction was not associated with recognition of the advertisements used during the campaign ${ }^{30}$.

The goal of this study was to evaluate the impact of a health promotion campaign aimed at increasing awareness about dementia risk reduction in middle-aged Dutch community-dwelling individuals. Specifically, we tested change in the level of awareness at the population-level before and after the campaign, variation between demographic groups, the effect of different approaches (mass media versus additional community-participation) and the use of eHealth supportive technology, in order to distil the lessons learned for future campaigns and policies. 


\section{Methods}

\section{Target population}

The target population were inhabitants of the Province of Limburg, the Netherlands, aged 40 to 75 years old $\left(558,535\right.$ people in total $\left.{ }^{31}\right)$. The Ethics Review Committee Psychology and Neuroscience (ERCPN) of Maastricht University approved this study (reference number 177-07-03-2017). All participants received an information letter and signed a digital informed consent form prior to participation.

\section{Awareness campaign}

The primary aim of this campaign (March 2018 - January 2019) was to increase awareness on dementia risk reduction. The secondary aim was to motivate people for behavioural change by means of eHealth. The campaign was developed by the Alzheimer Centre Limburg (ACL) at Maastricht University and Maastricht University Medical Centre+, in consultation with the Dutch Municipal Health Services (MHS) and the Department of Health Promotion of Maastricht University. An agency for strategy and design developed the campaign materials and website32. To maximize acceptance in the relatively young target population, a positive phrasing was chosen for the slogan ("We are our own medicine"), terminology (e.g., "brain health" rather than "dementia"), and campaign material visualizing the three campaign themes: "eat healthy", "exercise regularly", and "stay curious" (see Supplemental File 1 for examples). The campaign materials were discussed with stakeholders (Dutch Alzheimer's Association and an evaluation panel of potential endusers). We deliberately designed the campaign in a way that would address different people. We provided both a low-level and free app with short, simple text messages that appeared automatically on a daily basis, and provided an extensive website for background information and references to extra literature (e.g., website Dutch Alzheimer's Association). Two different campaign approaches were chosen in order to compare the outcome. A broad campaign was launched, targeting the public via mass media such as newspapers and social media (hereafter "population sample"). Three specific districts within the Province (Landgraaf-Schaesberg, Brunssum-Oost, and RoermondHoogvonderen) were chosen based on varying socio-economic status, from low to middle-high, and absence of other public health projects (hereafter "district sample"). We worked together with key-figures and facilities in that district, in order to meet the specific needs and wishes of that district. 
The eHealth platform

An online platform called MijnBreincoach ("MyBraincoach") was developed together with two software companies and made available as a mobile app and web portal to the general public during the campaign ${ }^{33}$. Users complete a " 12 item quick test" using the well-validated LIfestyle for BRAin Health (LIBRA) score $^{34}$. This predictive model consists of twelve modifiable risk and protective factors for dementia (e.g., smoking, physical inactivity, depression), and gives people insight into their personal dementia risk profile. Detailed information can be found in Supplemental File 2.

Pre- and post-campaign surveys

Two cross-sectional surveys were performed: one pre-campaign (September $2017^{18}$ ) and one post-campaign (February 2019). The pre- and post-campaign surveys took place in independent samples, in order to ensure that the potential increase in awareness was not caused by learning effects. The methodology and results of the pre-campaign survey have been described in more detail elsewhere ${ }^{18}$.

\section{Recruitment process}

The population samples were drawn by the MHS from participants of a previous national health survey ('Gezondheidsmonitor 2016') who had agreed to be contacted for future studies. A random selection of people (40-75 years) was invited to participate via email. The district samples were drawn from the municipal registry by the municipality or by the MHS, based on ZIP-codes and age and received a postal letter.

\section{Measurements}

Age, sex, marital status, educational level, self-reported knowledge of dementia and awareness of dementia risk reduction were assessed both at the pre- and post-assessment. Ten items from the British Social Attitudes (BSA) survey ${ }^{19}$ were used, translated into Dutch. To assess all twelve factors from the LIBRA-index, ${ }^{34}$ custom-made items were also included ${ }^{18}$. The post-campaign survey included additional items concerning exposure to the campaign (e.g., asking participants whether they recognized campaign material). The items on exposure to the campaign were placed after the items assessing awareness, thereby not influencing one's perspective on the possibility of dementia risk reduction. See Supplementary File 3 for the complete pre- and post-campaign survey. The primary outcome of awareness of dementia risk reduction was 
assessed as the difference between pre- and post-assessment in the proportion of people rejecting the statement 'There is nothing I can do to reduce my dementia risk'. We presented this statement in the Results section in a positive form for reasons of clarity ("dementia risk reduction is possible"). Secondary outcomes were changes in endorsement of the three campaign themes physical activity, cognitive activity and healthy diet.

\section{Statistical analyses}

Independent groups $t$-tests and $\chi^{2}$ tests were used to analyse differences between the pre- and post-campaign samples, and between population and district samples, and to investigate differences in the relation between sociodemographic variables and level of awareness and knowledge of risk and protective factors. Analyses were performed in Stata 13.1 (StataCorp, College Station, TX, USA), with the level of significance set at $\mathrm{p} \leq 0.05$ in two-tailed tests.

Patient and Public Involvement

There were no patients involved in this research. Members of the public were involved in the design and rollout of the public health campaign. 


\section{Results}

\section{Demographics}

The response rates of the pre- and post-campaign surveys were highly comparable (population pre-campaign $53.6 \%$, post-campaign $54.8 \%$; district pre-campaign $33.2 \%$, post-campaign $32.2 \%$ ). See Supplemental File 4, 5 a and $5 \mathrm{~b}$ for flowcharts of the recruitment process. Table 1 shows the characteristics of the total and the two separate samples. The characteristics of the districts can be found in Supplemental File 6. The total pre-assessment sample was highly comparable with the total post-assessment sample. As expected by design, the population sample was more highly educated than the district sample at the pre- $\left(\chi^{2}(2)=29.57, \mathrm{p} \leq 0.001\right)$ and post-assessment $\left(\chi^{2}(2)=17.41, \mathrm{p} \leq\right.$ $0.001)$. An overview of the forms of community engagement are displayed in Box 1. 


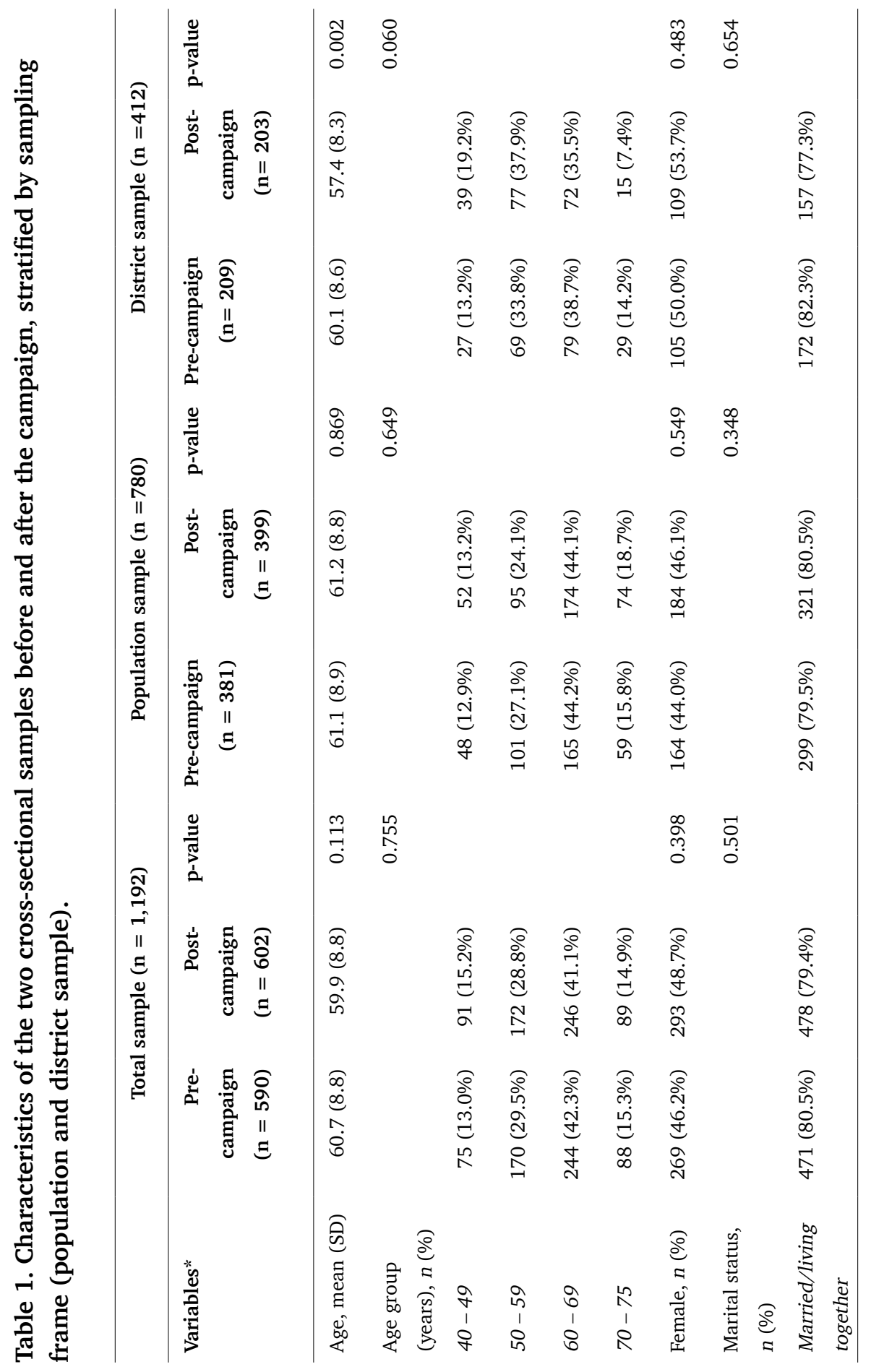




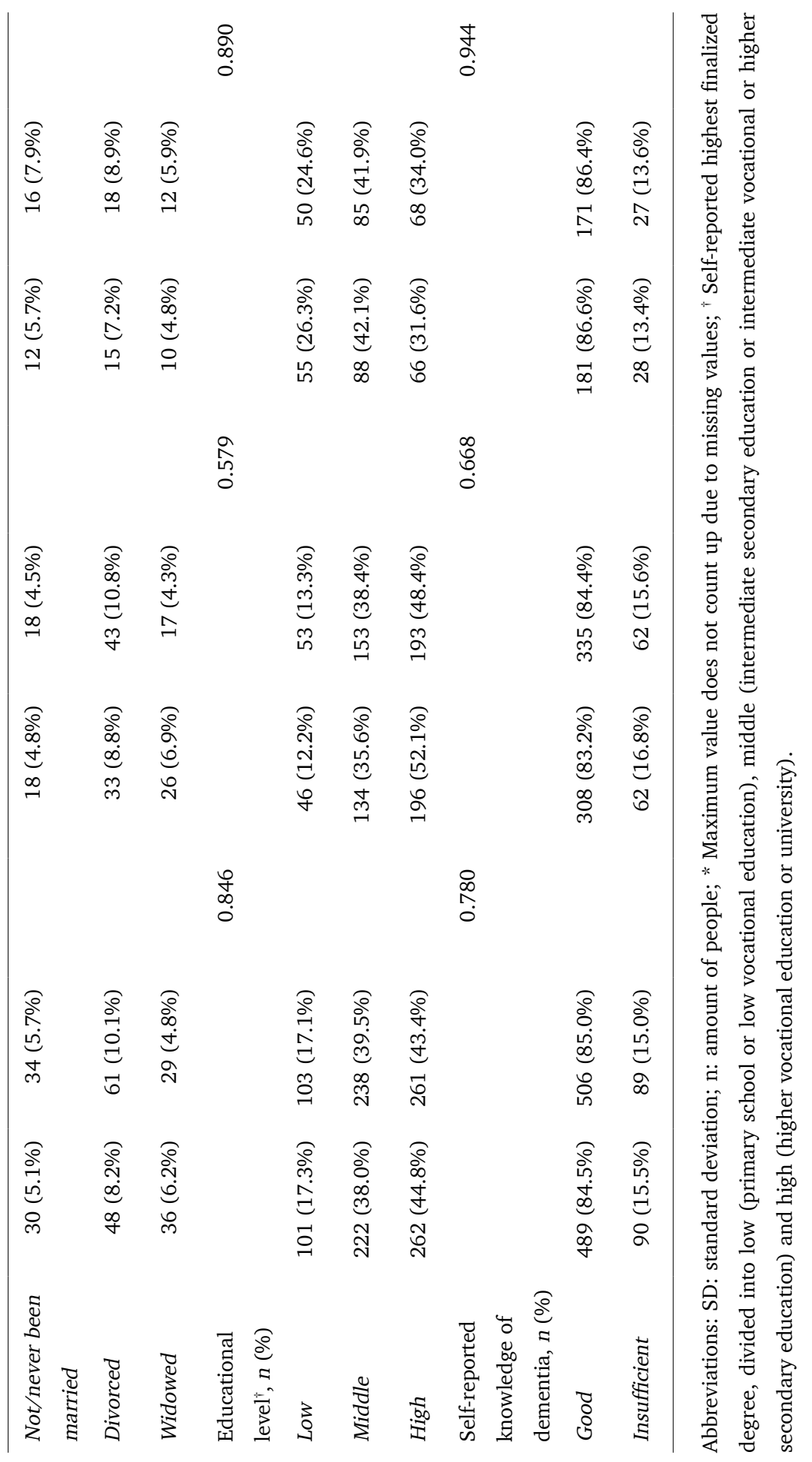




\section{Box 1. Forms of community engagement during the campaign} (March 2018 - January 2019).

Local $\begin{aligned} & \text { More than } 140 \text { stakeholders (municipalities, schools, health care } \\ & \text { engagement and } \\ & \text { support }\end{aligned}$
centres, companies) committed to this campaign by distributing
campaign material/messages and/or organizing public events $\quad \begin{aligned} & \text { Distribution of } 35,000 \text { campaign leaflets and more than } 1,000 \\ & \text { campaign posters (at more than } 400 \text { locations within the Province) } \\ & \text { - Organization of more than one public event per week (n=52; lecture, } \\ & \text { workshop or other community activities) }\end{aligned}$

\begin{tabular}{lll}
\hline $\begin{array}{l}\text { Campaign } \\
\text { website }\end{array}$ & $-\begin{array}{l}\text { More than 10,000 website visits } \\
\text { Online campaign posters are downloaded more than 5,500 times in } \\
\text { total }\end{array}$ \\
\hline
\end{tabular}

Media

- Over 65 media outlets (e.g., newspaper items, radio-interviews)

- Campaign tweets reached 200,000 people

- Facebook messages reached more than 15,000 people

eHealth platform - 9,000 downloads MijnBreincoach app

- Ministry of Health, Welfare and Sport in the Netherlands incorporated the app on their website on innovations in health care 
Exposure to the campaign

Of all post-campaign participants $(n=602), 20.0 \%$ reported to have heard of the campaign, $19.7 \%$ of the slogan, $21.8 \%$ about the eHealth platform, and $29.8 \%$ recognized one of the campaign materials (e.g., poster, flyer). Women heard more often about the eHealth platform $\left(27.5 \%\right.$ vs. $16.3 \% ; \chi^{2}(1)=9.75$, $p=.002)$ and recognized campaign material more often ( $34 \%$ vs. $25.8 \% ; \chi^{2}(1)$ $=4.23, p=.040$ ) compared to men. Lower educated participants recognized campaign material more often than more highly educated participants did (33.5\% vs. $\left.25.1 \% ; \chi^{2}(1)=4.28, p=.039\right)$.

Difference in level of awareness before and after the campaign (total sample) Figure 1 displays a pre- and post-campaign comparison of the percentage of participants agreeing that dementia risk reduction is possible, and the percentage of participants identifying the three campaign themes. No difference in the primary outcome of awareness of dementia risk reduction was observed $\left(X^{2}(1)=1.27, p=0.260\right)$. Cognitive activity was identified most often as being protective against dementia at both pre- $(79.4 \%)$ and post-assessment (80.4\%), but there was no increase in awareness $\left(\chi^{2}(1)=0.17, p=0.677\right)$. A modest increase in awareness was observed for physical activity (7.6\% increase; $\left.\chi^{2}(1)=7.48, p=.006\right)$ and healthy diet (10.5\% increase; $\chi^{2}(1)$ $=12.37, p \leq 0.001)$. More highly educated participants were more aware of dementia risk reduction compared to lower educated participants, both in the pre-assessment (low 18.2\%, middle 38.9\%, high 59.4\%; $\chi^{2}(2)=53.46, p \leq$ 0.001 ) and post-assessment (low $29.3 \%$, middle $33.9 \%$, high $52.3 \% ; \chi^{2}(2)=$ $24.15, p \leq 0.001)$. The same applies to the identification of the three campaign themes. In men, level of awareness decreased slightly with $8 \%\left(\chi^{2}(1)=3.89\right.$, $p=.049$ ), but they identified the campaign theme "eat healthy" more often over time $\left(\chi^{2}(1)=10.99, p=.001\right)$. The level of awareness remained stable over time in women $\left(\chi^{2}(1)=0.09, p=.770\right)$, participants under the age of 65 years $\left(\chi^{2}(1)=0.78, p=.377\right)$ and participants aged 65 and above $\left(\chi^{2}(1)\right.$ $=1.46, p=.227)$, but over time, the theme "exercise regularly" was identified more often by participants under the age of 65 years $\left(9.4 \%\right.$ increase; $\chi^{2}(1)=$ 7.13, $p=.008)$. 


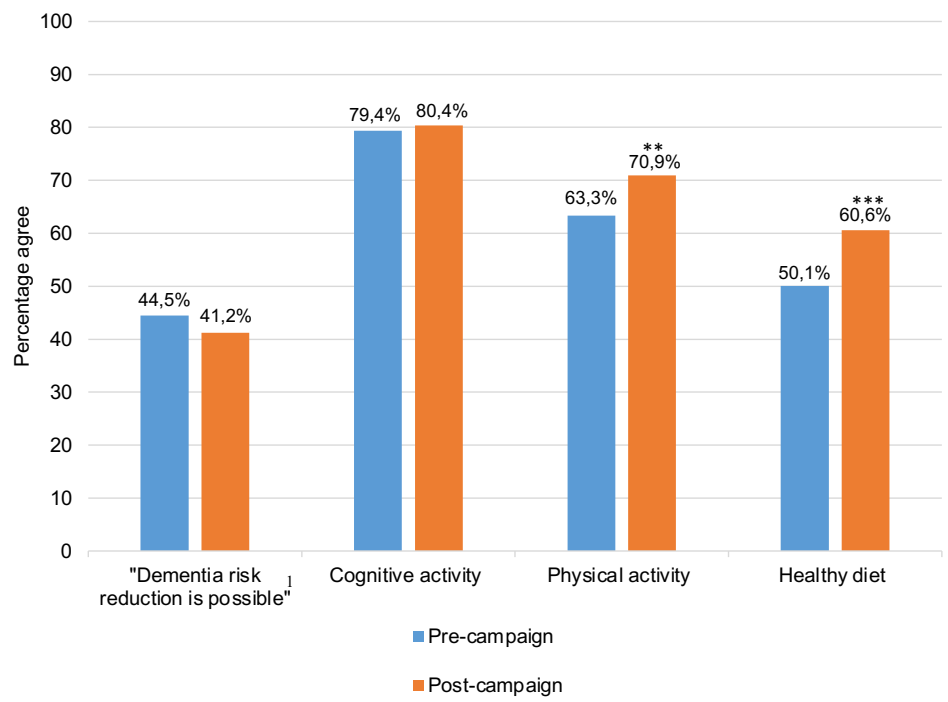

Figure 1. Pre- $(n=590)$ and post- $(n=602)$ campaign comparison of the total sample. Percentage agreeing with the statement that dementia risk reduction is possible, and percentage identifying the three target risk factors/themes of the campaign. Maximum values and percentages do not count up due to missing values. ${ }^{1}$ Original statement presented to participants: "There is nothing I can do to reduce my dementia risk". " $p<.05 ; * * p<.01 ; * * * p<.001$.

\section{Differences between the two campaign approaches}

No significant difference in level of awareness was found for both the population $\left(47.1 \%\right.$ to $\left.40.5 \% ; \chi^{2}(1)=3.39, p=0.066\right)$ and district sample (39.9\% to $\left.42.7 \% ; \chi^{2}(1)=0.33, p=0.565\right)$. Compared to pre-assessment, cognitive activity was not identified more often as a protective factor for dementia at post-assessment, either in the population $\left(79.9 \%\right.$ to $81.8 \% ; \chi^{2}(1)$ $=0.43, p=0.510)$ or district sample $\left(78.5 \%\right.$ to $77.7 \% ; \chi^{2}(1)=0.04, p=$ $0.844)$. Physical activity was identified more often in the population $(65.6 \%$ to $\left.73.3 \% ; \chi^{2}(1)=5.14, p=.023\right)$, but not in the district sample $(59.2 \%$ to $\left.66.3 \% ; \chi^{2}(1)=2.17, p=0.141\right)$. Healthy diet was identified more often in both the population $\left(51.7 \%\right.$ to $\left.62.3 \% ; \chi^{2}(1)=8.23, p=.004\right)$ and district sample $\left(47.3 \%\right.$ to $\left.57.4 \% ; \chi^{2}(1)=3.99, p=.046\right)$. An increase was found of the LIBRA factors low-to-moderate alcohol use $\left(26.9 \%\right.$ to $38.4 \% ; \chi^{2}(1)=6.07$, $p=.014)$, obesity ( $19.9 \%$ to $\left.28.4 \% ; \chi^{2}(1)=3.91, p=.048\right)$ and smoking $\left(29.3 \%\right.$ to $\left.42.9 \% ; \chi^{2}(1)=8.15, p=.004\right)$ in the district sample. More highly 
educated participants were more aware of dementia risk reduction compared to lower educated participants, both in the pre-assessment (population low $13.7 \%$, middle $39.1 \%$, high $60.2 \% ; \chi^{2}(2)=36.27, p \leq 0.001$; districts low $21.8 \%$, middle $38.6 \%$, high $\left.56.9 \% ; \chi^{2}(2)=15.41, p \leq 0.001\right)$ and the postassessment (population low $31.4 \%$, middle $30.0 \%$, high $51.0 \% ; \chi^{2}(2)=17.49$, $p \leq 0.001$; districts low $27.1 \%$, middle $41.0 \%$, high 55.9\%; $\chi^{2}(2)=9.72, p$ $=.008)$. In those with a low level of education in the population, an increase in awareness of dementia risk reduction was observed $\left(17.7 \%\right.$ increase; $\chi^{2}(1)=$ $4.18, p=.041)$, and for the campaign themes physical activity (22\% increase; $\left.\chi^{2}(1)=4.35, p=.037\right)$ and healthy diet $\left(25.3 \%\right.$ increase; $\chi^{2}(1)=5.79, p$ $=.016)$.

Exposure to the campaign and level of awareness in the total post-campaign sample $(n=602)$

Awareness of dementia risk reduction was higher for post-campaign participants who reported to have heard compared to those who have not heard of the campaign $\left(51.4 \%\right.$ vs. $\left.37.9 \% ; \chi^{2}(1)=6.52, p=.011\right)$, the campaign slogan $\left(53.3 \%\right.$ vs. $\left.37.2 \% ; \chi^{2}(1)=9.07, p=.003\right)$ and the eHealth platform $(54.8 \%$ vs. $\left.36.6 \% ; \chi^{2}(1)=12.39, p \leq 0.001\right)$. Campaign materials were more often recognized in the districts (35.2\%) than in the population sample $(26.8 \%$; $\left.\chi^{2}(1)=3.92, p=.048\right)$. More than a third $(37.2 \%)$ expressed to have become more conscious of lifestyle being related to their brain health, and $30.4 \%$ stated to have engaged in a brain-healthy lifestyle. Physical activity (45.8\%), eating healthy (40.9\%), and weight management (39.4\%) were most often engaged in during the past year.

\section{Self-reported knowledge of dementia}

Figure 2 displays the level of awareness by self-reported general knowledge of dementia in the post-assessment sample. Participants who stated that their general knowledge of dementia was considerable or good were more aware of dementia risk reduction than participants with self-reported insufficient general knowledge $\left(\chi^{2}(1)=6.48, p=.011\right)$. The same applied to the identification of the risk/protective factors physical activity $\left(\chi^{2}(1)=4.59\right.$, $p=.032)$, healthy diet $\left(\chi^{2}(1)=7.32, p=.007\right)$, smoking $\left(\chi^{2}(1)=8.18\right.$, $p=.004)$, depression $\left(\chi^{2}(1)=5.44, p=.020\right)$, diabetes $\left(\chi^{2}(1)=8.31, p\right.$ $=.004)$, and hypercholesterolemia $\left(\chi^{2}(1)=6.60, p=.010\right)$. 


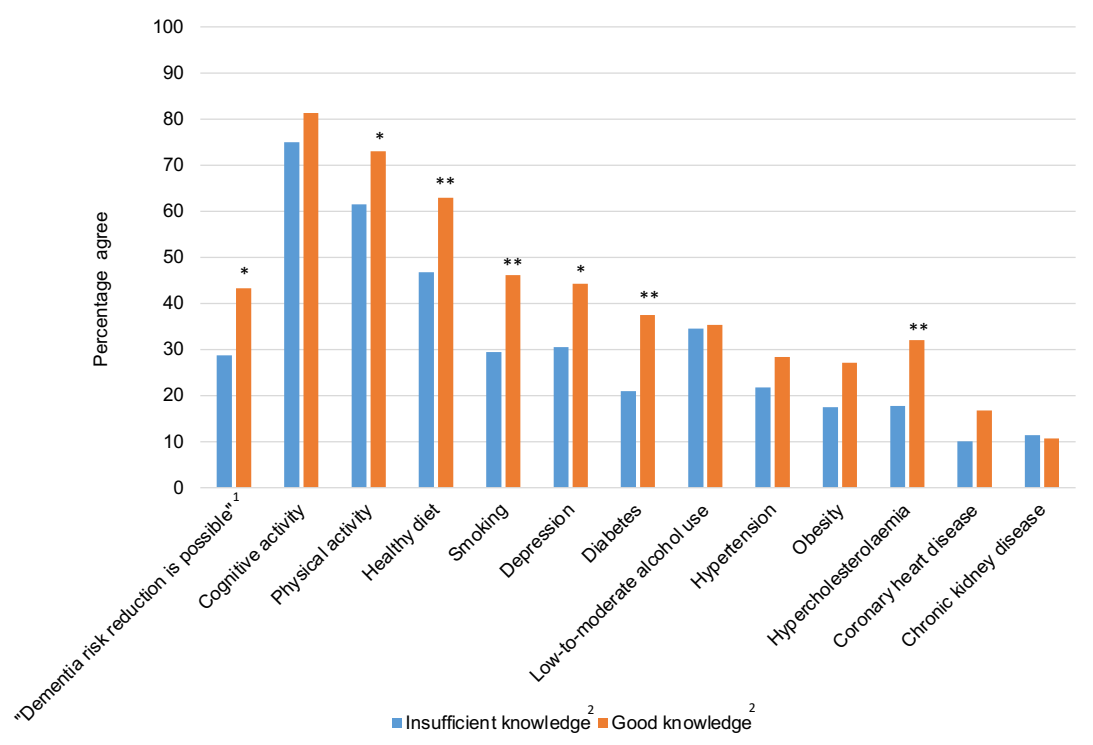

Figure 2. Level of awareness by self-reported general knowledge of dementia in the postassessment sample $(n=602)$. The percentages reflect the percentage of participants who agreed that a particular factor is a risk or protective factor for dementia. Maximum values and percentages do not count up due to missing values. ${ }^{1}$ Original statement presented to participants: "There is nothing I can do to reduce my dementia risk". ${ }^{2}$ Self-reported knowledge of dementia, divided into "Insufficient knowledge" (answering options "I don't know", "Nothing at all" and "Not very much") and "Good knowledge" ("Some", "Quite a lot" and "A great deal"). * $p<.05 ; * * p<.01 ; * * * p<.001$.

\section{The eHealth platform}

Anonymous user-tracking showed that the 12-item "quick test" was completed more than 13,300 times by people from the general public during the campaign. The mean age of this group was 57 years (SD 14.3; range 18-94y), 68\% were female and $76 \%$ were higher educated (i.e., higher vocational education or university). Room for improvement (according to self-reported presence or absence of risk/protective factors) was highest for the LIBRA factors hypertension, hypercholesterolemia, and alcohol consumption. Almost 36\% $(n=4,755)$ created an account and completed the more comprehensive administration (mean age 57 years, $72 \%$ female, $78 \%$ higher educated). Room for improvement, based on the extensive LIBRA administration using validated questionnaires, was highest for physical inactivity, adherence to a Mediterranean diet, and cognitive activity. These were also the factors that were chosen most often for receiving daily notifications. 


\section{Discussion}

This paper presents the results of the first health promotion campaign in the Netherlands aimed at increasing awareness of dementia risk reduction in middle-aged, community-dwelling individuals. In general, this study was not successful since no population-level change in awareness was observed. However, two out of the three campaign themes were identified more often after the campaign. People exposed to the campaign, its slogan and the eHealth platform were significantly more aware of dementia risk reduction and the three campaign themes. Participants from the district campaign recognized campaign material and the eHealth platform more often.

Unfortunately, awareness of dementia risk reduction and knowledge of most LIBRA factors did not increase. Several reasons might exist. This campaign did not use national mass media, in contrast to a population-based awareness campaign in Ireland that did find a significant increase in awareness of dementia risk reduction ${ }^{30}$. Due to a limited budget and resources, the coverage of our campaign might have been insufficient to reach population-level increase in awareness. Interestingly, our study did find an increase in awareness in those who reported to have been exposed to the campaign, while the Irish study could not differentiate between the exposed and non-exposed group ${ }^{30}$. Women stated more often than men to be exposed to our campaign material and to have visited the eHealth platform, which is in line with previous studies stating that women participate more than men in health campaign $s^{35}$. However, this did not translate in an increase in awareness in women at post-assessment. In addition, it could be that the statement to assess awareness was too complex ("there is nothing I can do to reduce my dementia risk"). A simpler, positively formulated statement might have been more suitable for our purpose. The statement used was taken from the BSA $2015^{19}$ in order to compare dementia literacy between the United Kingdom and the Netherlands. Furthermore, there was no higher endorsement of the protective factor of cognitive activity after the campaign. This might be explained by a ceiling effect, as many people already considered it to be a protective factor at baseline.

Strengths of this study include the extensive pre- and post-campaign surveys, in which we used multiple items to assess awareness of dementia risk reduction in general, and specific risk and protective factors. Furthermore, we used large independent samples and comparable methodology to a previous study assessing awareness of dementia risk reduction ${ }^{19}$. Next, the intervention part 
(awareness campaign) of this study was designed in consultation of experts, addressed, in line with the WHO guidelines ${ }^{5}$, multiple dementia risk factors and collaborated with stakeholders in a multidisciplinary approach ${ }^{5}$. The involvement of stakeholders created a "snowball effect", as they communicated the campaign message via their own channels (see Box 1). Also, although the basic framework was set beforehand, the flexible design of the campaign made it possible to alter strategies along the way.

This study, however, also had limitations. First, this study was not inclusive regarding non-Dutch speaking individuals and individuals without internet access. Furthermore, the population samples were drawn from participants from a previous survey interested in future research. This could have led to a pre-selection of people interested in scientific research and health. Last, the restrained resources of the team (e.g., to contact relevant stakeholders) limited the reach and effect of the campaign. These limitations were already acknowledged beforehand. This campaign was developed as a proof-of-concept study in a naturalistic setting, investigating campaign strategies and the extent of involvement of the community.

\section{Recommendations for future campaigns}

In general, involvement of the community is an important determinant of success. Further, as this study shows that individuals with self-reported sufficient knowledge of dementia are more aware of dementia risk reduction, it is recommended to incorporate such a campaign into a general dementia campaign, or even a broad health promotion campaign, given the overlapping risk factors for cardiovascular disease and diabetes. In fact, incorporating lifestyle recommendations of various non-communicable diseases is one of the recommendations of the $\mathrm{WHO}^{5}$. Furthermore, it is important to tailor health messages to specific subgroups (e.g., based on educational level, age, sex, high/ low risk group). Their needs, wishes and barriers to engage in a brain-healthy lifestyle should be further explored, both prior to the execution of a campaign and as a post-campaign evaluation, for example by qualitative research. To illustrate, comparable to earlier studies ${ }^{22-24}$, our study showed that dementia risk reduction literacy was higher in more highly educated participants. However, campaign material was recognized more often and awareness only improved in the lower educated group (particularly in the population sample). The campaign was designed with differences in health literacy and socioeconomic status in mind (e.g., content checking by the MHS). Also, it was 
striking that campaign activities (e.g., lectures, workshops) were particularly visited by the older half (60-75 years) of the targeted population, despite our efforts in providing information online and using terms as "brain health" instead of "dementia risk". Reaching younger individuals, with a positively framed message on the potential of dementia risk reduction, is important to take into consideration. Next, it should be noted that increasing awareness is an essential yet insufficient step towards behavioural change. Altering complex and entrenched behaviours is very difficult, and unlikely to be sufficiently affected by this small-scale campaign. This was done to some extent by prompting people with low-level, positive messages on how to engage in brain-healthy activities. Yet, the main focus of this campaign was increasing awareness and not behavioural change.

\section{Conclusion}

This study was not able to reach a population-level increase of awareness of dementia risk reduction, but did increase awareness and willingness to take action in those exposed. Future campaigns should scale up to maximize exposure and engagement in the population. More insight is needed on how increasing awareness may trigger lifestyle behaviour. 


\section{References}

1. Livingston G, Sommerlad A, Orgeta V, et al. Dementia prevention, intervention, and care. Lancet 2017;390:2673-734.

2. Patterson C. World Alzheimer Report 2018. London: Alzheimer's Disease International, 2018.

3. Lincoln P, Fenton K, Alessi C, et al. The Blackfriars Consensus on brain health and dementia. Lancet 2014;383:1805-06.

4. Norton S, Matthews FE, Barnes DE, et al. Potential for primary prevention of Alzheimer's disease: an analysis of population-based data. Lancet Neurol 2014;13:788-94.

5. Risk reduction of cognitive decline and dementia: WHO guidelines. Geneva: World Health Organization, 2019.

6. Ngandu T, Lehtisalo J, Solomon A, et al. A 2 year multidomain intervention of diet, exercise, cognitive training, and vascular risk monitoring versus control to prevent cognitive decline in at-risk elderly people (FINGER): a randomised controlled trial. Lancet 2015;385:2255-63.

7. Moll van Charante EP, Richard E, Eurelings LS, et al. Effectiveness of a 6-year multidomain vascular care intervention to prevent dementia (preDIVA): a clusterrandomised controlled trial. Lancet 2016;388:797-805.

8. Andrieu S, Guyonnet S, Coley N, etal.Effect of long-term omega 3 polyunsaturated fatty acid supplementation with or without multidomain intervention on cognitive function in elderly adults with memory complaints (MAPT): a randomised, placebo-controlled trial. Lancet Neurol 2017;16:377-89.

9. Kivipelto $\mathrm{M}$, Ngandu $\mathrm{T}$, Laatikainen $\mathrm{T}$, et al. Risk score for the prediction of dementia risk in 20 years among middle aged people: a longitudinal, populationbased study. Lancet Neurol 2006;5:73541.

10. Rosenberg A, Ngandu T, Rusanen M, et al. Multidomain lifestyle intervention benefits a large elderly population at risk for cognitive decline and dementia regardless of baseline characteristics: The FINGER trial. Alzheimers Dement 2018;14:263-70.

11. Kivipelto M, Mangialasche F, Ngandu T. Lifestyle interventions to prevent cognitive impairment, dementia and Alzheimer disease. Nat Rev Neurol 2018;14:653-66.

12. Deckers $K$, Kohler S, van Boxtel M, et al. Lack of associations between modifiable risk factors and dementia in the very old: findings from the Cambridge City over75s cohort study. Aging Ment Health 2017;22:1272-78.

13. Vos SJB, van Boxtel MPJ, Schiepers OJG, et al. Modifiable Risk Factors for Prevention of Dementia in Midlife, Late Life and the Oldest-Old: Validation of the LIBRA Index. $J$ Alzheimers Dis 2017;58:537-47.

14. Prince $\mathrm{M}$, Ali $\mathrm{G}-\mathrm{C}$, Guerchet $\mathrm{M}$, et al. Recent global trends in the prevalence and incidence of dementia, and survival with dementia. Alzheimers Res Ther 2016;8:23-23. 
15. Roehr S, Pabst A, Luck T, et al. Is dementia incidence declining in high-income countries? A systematic review and metaanalysis. Clin Epidemiol 2018;10:123347.

16. Satizabal CL, Beiser AS, Chouraki V, et al. Incidence of Dementia over Three Decades in the Framingham Heart Study. N Engl J Med 2016;374:523-32.

17. Livingston G, Huntley J, Sommerlad A, et al. Dementia prevention, intervention, and care: 2020 report of the Lancet Commission. Lancet 2020;396(10248):413-46.

18. Heger I, Deckers K, van Boxtel M, et al. Dementia awareness and risk perception in middle-aged and older individuals: baseline results of the MijnBreincoach survey on the association between lifestyle and brain health. BMC Public Health 2019;19:678.

19. Marcinkiewicz A, Reid S. Attitudes to dementia: Findings from the 2016 British Social Attitudes survey London: NatCen Social Research, 2016.

20. Cations M, Radisic G, Crotty M, et al. What does the general public understand about prevention and treatment of dementia? A systematic review of population-based surveys. PLoS One 2018;13:e196085.

21. Liu D, Cheng G, An L, et al. Public Knowledge about Dementia in China: A National WeChat-Based Survey. Int $J$ Environ Res Public Health 2019;16:4231.

22. de Veer AJ, Peeters JM, Brabers AE, et al. Determinants of the intention to use e-Health by community dwelling older people. BMC Health Serv Res
2015;15:103.

23. Eakin EG, Bull SS, Glasgow RE, et al. Reaching those most in need: a review of diabetes self-management interventions in disadvantaged populations. Diabetes Metab Res Rev 2002;18:26-35.

24. Luten $\mathrm{KA}$, Dijkstra $\mathrm{A}$, de Winter AF, et al. Developing a community-based intervention for Dutch older adults in a socioeconomically disadvantaged community. Health Promot Int 2019;34:567-80.

25. Askari N, Bilbrey AC, Garcia Ruiz I, et al. Dementia Awareness Campaign in the Latino Community: A Novel Community Engagement Pilot Training Program with Promotoras. Clin Gerontol 2018;41:20008.

26. Moorhouse P, Hamilton LM. Not if, but when: impact of a driving and dementia awareness and education campaign for primary care physicians. CGJ 2014;17:705.

27. Kim S, Werner P, Richardson A, et al. Dementia Stigma Reduction (DESeRvE): Study protocol for a randomized controlled trial of an online intervention program to reduce dementia-related public stigma. Contemp Clin Trials Commun 2019;14:100351.

28. Werner P, Kermel Schiffman I. Exposure to a national multimedia Alzheimer's disease awareness campaign: Assessing stigmatic beliefs towards persons with the disease. Int $J$ Geriatr Psychiatry 2018;33:e336-e42.

29. Farrow M. User perceptions of a dementia risk reduction website and its promotion 
of behavior change. JMIR Res Protoc 2013;2:e15.

30. Hickey D. The impact of a National Public Awareness Campaign on Dementia Knowledge and Help-seeking Intention in Ireland. Dublin: Health Service Executive: 2019.

31. Centraal Bureau voor Statistiek (CBS) Statline; [Available at: https:// opendata.cbs.nl/statline/\#/CBS/nl/ dataset/03759ned/table? $\mathrm{dl}=26570$. Accessed 12-09-2019.

32. Campaign website [Available at: https:// www.wezijnzelfhetmedicijn.nl/. Accessed 19-12-2019.

33. Online platform MijnBreincoach [Available at: https://www. mijnbreincoach.eu/. Accessed 19-122019.

34. Deckers K, van Boxtel MP, Schiepers OJ, et al. Target risk factors for dementia prevention: a systematic review and Delphi consensus study on the evidence from observational studies. Int $J$ Geriatr Psychiatry 2015;30:234-46.

35. Compernolle S, De Cocker K, Lakerveld J, et al. A RE-AIM evaluation of evidencebased multi-level interventions to improve obesity-related behaviours in adults: a systematic review (the SPOTLIGHT project). Int $J$ Behav Nutr Phys Act 2014;11:147. 
Raising awareness for dementia risk reduction through a public health campaign 

Chapter 6

Appreciation of an mHealth tool to increase knowledge and beliefs and attitudes for dementia risk reduction: a pre-post proof-of-concept study

Irene Heger, Kay Deckers, Martin van Boxtel, Marjolein de Vugt, Frans Verhey, Anke Oenema, Sebastian Köhler

Submitted 



\section{Abstract}

Background: The number of people with dementia is increasing worldwide and to date there is no curative treatment, making it one of the biggest public health challenges for the upcoming decades. Yet, health- and lifestyle factors account for a substantial part of all dementia cases worldwide, which opens up the opportunity of primary prevention of dementia. The general public is still largely unaware of the potential of dementia risk reduction. mHealth interventions can potentially contribute to improving knowledge and motivation for dementia risk reduction in a low-cost and scalable way.

Objectives: To evaluate two versions (standard and extended) of the mobile app "MijnBreincoach" ("MyBraincoach") on usage pattern, appreciation, and beliefs and attitudes related to dementia risk behaviours. Both versions aimed at improving knowledge and identifying dementia risk behaviour. The extended version also aimed at increasing beliefs and attitudes related to identified dementia risk behaviours.

Methods: A pre-post-design was used to assess usage pattern (usage-tracking data), appreciation, and seven constructs of beliefs and attitudes related to dementia risk reduction (the MCLHB-DRR Scale). Participants were community-dwelling middle-aged adults (40-75 years) from the Netherlands. Participants used one of the two versions of the app. Linear mixed models were used, adjusted for age, sex and educational level.

Results: Of all participants $(n=299), 190(63.6 \%)$ completed the postevaluation and 167 (55.9\%) had installed the app. The most reported reason for non-use was technical problems during installation and use (47.1\%). Women installed the app more often than men $\left(63.1 \%\right.$ vs. $48.7 \% ; \chi^{2}$ (1) $=6.304, P=.01)$. Of all participants who responded to the follow-up assessment and reported to have used the app $(n=102), 78.4 \%$ stated that the app provided a positive approach towards brain health and $>80 \%$ felt better informed about dementia risk reduction. Usage-tracking showed that only 16 of the 101 participants (15.8\%) who were randomized to the extended app used the main additional feature. An intention-to-treat analyses revealed no pre-post changes in beliefs and attitudes (perceived susceptibility $B=-0.07$, $P=.56$; perceived severity $\mathrm{B}=-0.26, P=.18$, perceived benefits $\mathrm{B}=-0.13$, $P=.20$, perceived barriers $\mathrm{B}=0.23, P=.16$, cues to action $\mathrm{B}=0.09, P=.60$, 
general health motivation $\mathrm{B}=0.01, P=.92$, and self-efficacy $\mathrm{B}=-0.05, P=.62$ ). No significant differences were found between the two versions of the app. Female, younger $(<60 \mathrm{y})$ and low-to-middle educated participants evaluated the app most positively.

Conclusions: Most participants evaluated the app positively and stated to have increased their knowledge on the topic. Usage of the extended functions of the app was low, which may explain why modification of beliefs and attitudes towards behavioural change did not change. Improving the use of the app is important to take into consideration in future studies. 


\section{Introduction}

Dementia is a syndrome characterized by progressive deterioration of cognitive abilities, leading to disturbances in daily activities and increased dependency. The most common underlying cause of dementia is Alzheimer's disease, while mixed forms (e.g., neurodegeneration and vascular damage) often co-exist ${ }^{1,2}$. To date, there is no curative treatment available and the number of people with dementia is rising worldwide, making dementia one of the biggest challenges for public health in the upcoming decades that requires good management ${ }^{3}$. There is increasing evidence that dementia is associated with modifiable risk and protective factors, such as smoking, obesity and physical inactivity ${ }^{2,4-6}$, which opens up the opportunity for primary prevention of dementia. This is underscored by the 2020 report of the Lancet Commission on Dementia Prevention, Intervention, and Care that concluded that a total of 12 modifiable factors account for around $40 \%$ of the people with dementia worldwide ${ }^{3}$. In addition, the World Health Organization recently published guidelines for risk reduction of cognitive decline and dementia ${ }^{1}$. In order to minimalize brain damage due to long time risk factor exposure, midlife seems a suitable age bracket to target most of these factors $4,7,8$.

Nevertheless, the public is still largely unaware of the relationship between lifestyle- and health-related factors and dementia risk ${ }^{9-11}$. A systematic review of population-based surveys from 2018 concluded that almost half of all respondents believed that dementia is non-preventable ${ }^{12}$, which can pose barriers to engage in behaviour change to reduce dementia risk. Particular gaps of knowledge exist with regard to the contribution of cardiovascular risk factors to dementia risk, such as hypercholesterolemia, midlife obesity and smoking 9, 12, 13. Thus, from a public health perspective, important steps are to increase knowledge, awareness and, subsequently, motivation to make brain healthy lifestyle changes $4,10,14-16$.

Using eHealth and mHealth for this purpose offers several advantages over traditional, face-to-face public health methods. For example, the larger reach (over $92 \%$ of the Dutch population was in the possession of a smartphone in $2019^{17}$ ), the easy accessibility, increased adherence, low costs (e.g., no trained staff and location needed) ${ }^{18,19}$ and potentially less socioeconomic disparity ${ }^{20}$. In a randomized clinical trial of patients with coronary heart disease, receiving short motivational text messages four times a week for six months led to significant reductions in risk factor exposure levels ${ }^{20}$. Raising awareness about 
the relationship between lifestyle and brain health and informing people about their personal room-for-lifestyle-improvement in a similar way might be a costeffective and scalable approach to help people make the behavioural changes for dementia risk reduction ${ }^{12,21}$. In fact, one of our own studies from 2019 showed that $54 \%$ of the study sample from the general population (40-75 years old) would like to use an app to learn more about brain health, and $27 \%$ is willing to consider using it ${ }^{9}$.

A recent systematic literature review on 14 web-based multi-domain lifestyle programs to improve brain health found beneficial effects on dementia risk score and cognitive test performance ${ }^{22}$. However, interventions were very heterogeneous regarding program content (tailoring based on current lifestyle or goals), duration ( $4-204$ weeks) and targeted lifestyle factors (two to nine factors), while a detailed description of evaluation methods and results was often lacking. Reporting a clear overview of applied methodology and results is advised in order to compare the effectiveness and sustainability ${ }^{22}$. Moreover, making web-based interventions available to the general public is important to bridge the gap between scientific research and society.

This proof-of-concept study aims to evaluate the mHealth intervention MijnBreincoach ("MyBraincoach"), that uses the same dementia risk score to identify room-for-lifestyle-improvement as one of the web-based interventions from the review and was now made available without charge to the general public in the Netherlands. MijnBreincoach aims to improve knowledge of brain health and motivation for behavioural change in the general population through tailored lifestyle advice. We will test two versions (standard and extended) of the app on usage pattern, appreciation and beliefs and attitudes related to motivation to modify risk behaviours. 


\section{Methods}

\section{Study Design}

This study combined two studies regarding the MijnBreincoach app that took place in May - July 2018 and in October - December 2019. Both studies involved a pre-post study design. In the 2018-study, participants used the standard version of the app (see Method Section, The MijnBreincoach mobile app (standard version)). After completion of this study, another version of the app was developed that had extended functions (see Method Section, Features of the extended app). During the 2019-study participants were either randomized to the group that used the standard version of the app, or to the group that used the extended version. For the current analyses, data from both studies were combined for a larger sample size and to allow comparison between the two versions of the app. The Ethics Review Committee Psychology and Neuroscience (ERCPN) of Maastricht University approved the 2018 study (ERCPN- 185_01_11_2017) and the 2019 study (reference number ERCPN-208_08_05_2019).

\section{Participants}

Eligible for this study were community-dwelling, middle-aged (40 - 75 years) individuals without dementia (self-report), and with access to a smartphone with Internet connection, living in the Netherlands. The study aimed to include 100 participants in the 2018-study and 200 participants $(n=100$ standard app, $n=100$ extended app) in the 2019-study. Participants were sampled from responders of previous dementia risk awareness surveys ${ }^{9,23}$ who agreed to participate in future research. For the 2019-study, we made use of additional recruitment strategies via an online advertisement on the website of the Dutch Brain Foundation (Hersenstichting) and via a newsletter for staff members of a large health care institution in the Province of Limburg, the Netherlands (Meander Groep Zuid-Limburg). In this advertisement, individuals were asked if they were willing to participate in a study of Maastricht University regarding "an application related to brain health and lifestyle". Besides eligibility criteria, no more information was given on the purpose of this study or the app.

\section{Procedure}

Figure 1 provides a flowchart of the recruitment process, actual app use and survey response of the total sample and Multimedia Appendix 1 provides an overview per study year. All eligible individuals were contacted via e-mail, 
with information on the study (including information on length of time of the survey and anonymous data storage) and a hyperlink to the online informed consent form and baseline questionnaire using Lime Survey software (see Method Section, Measures). For the participants of the 2019-study, participants were randomized based on computer generated random numbers by an independent researcher to either the standard or extended version of the app. After completion of the baseline questionnaire, participants received an email with detailed information on how to install the app. They were requested to use the app on a daily basis for the next twelve weeks. Standard app users could install the app via their general app store, and extended app users could install the app via a secured hyperlink (Android) or by installing the TestFlight app first (iOS). Participants could email or call the research team with questions and remarks concerning the installation process and use of the app. Every two weeks a reminder email was sent to all participants encouraging them to use the app. Halfway through the intervention period (after 6 weeks), a personal email was sent to all participants who had not yet installed the app. After the intervention period of 12 weeks, all participants received an e-mail with a personal and secured hyperlink to the online follow-up questionnaire. All participants who completed the study received a gift card of 20 euro. Data was anonymized, stored on a protected server of the research setting and access was only granted to the authors that performed the statistical analyses (IH, SK). 


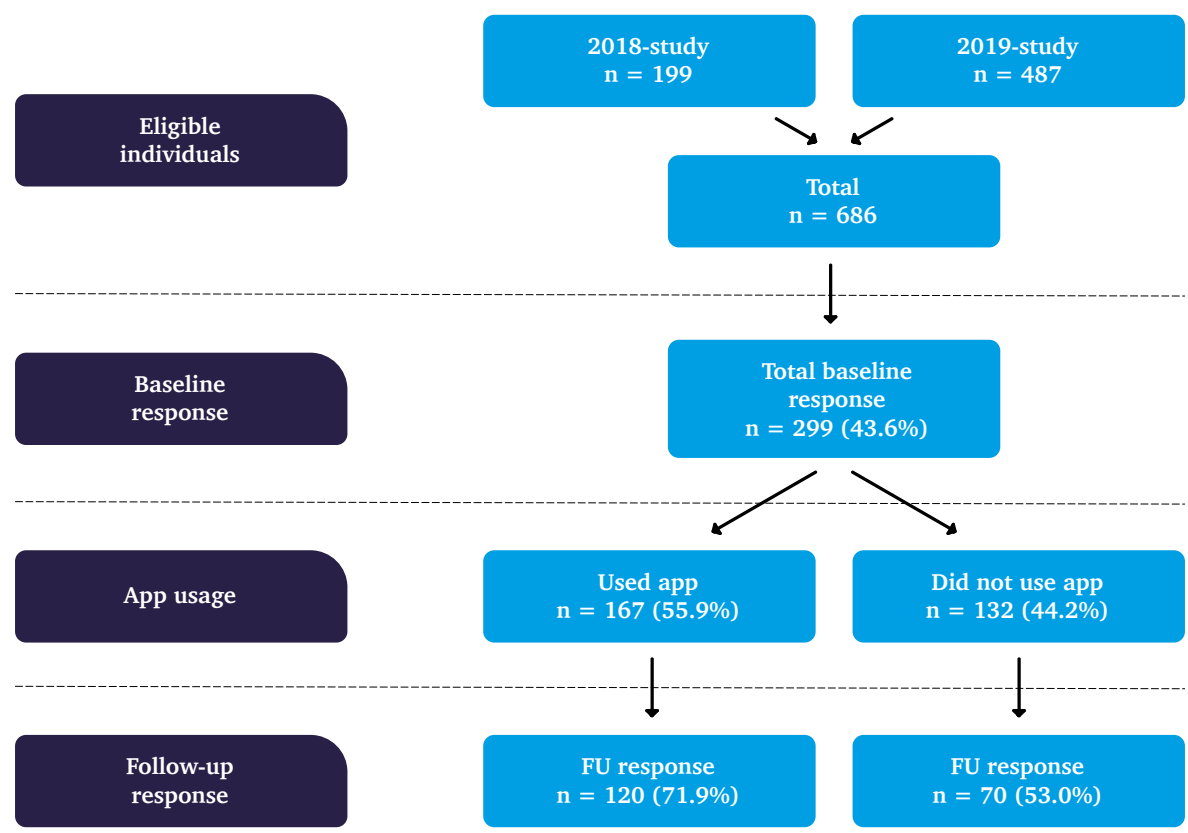

Figure 1. Flowchart of the recruitment process, actual app use and survey response of the total sample. Recruitment via: eligible individuals from responder samples of surveys evaluating a health campaign and advertisement on website Dutch Brain Foundation and newsletter large health care institution in the south of the Netherlands.

\section{Measures}

This study made use of usage tracking data and online questionnaires before and after the intervention to assess actual use, reasons for non-use and dropout, appreciation of the app (including perceived knowledge gain) and (changes in) beliefs and attitudes towards behavioural change for dementia risk reduction.

\section{Usage tracking data}

An export of usage tracking data was used to assess the usage pattern of all participants (see Figure 1 and Multimedia Appendix 1, app usage). This provided data for each individual participant on the dementia risk profile, lifestyle topics chosen to receive daily notifications, the number of daily notifications received and the number of days the app was used. 
Online questionnaires before and after the intervention period

Prior to and after the intervention period, participants completed an online questionnaire (see Figure 1 and Multimedia Appendix 1, baseline and follow-up response). The online questionnaire at baseline included items on demographics (i.e., age, sex, educational level, marital status), mobile operating system (iOS or Android) and beliefs and attitudes towards behavioural change for dementia risk reduction by means of the Motivation to Change Lifestyle and Health Behaviours for Dementia Risk Reduction (MCLHB-DRR) Scale ${ }^{24}$. This 27-item scale assesses seven factors that reflect dimensions of the Health Belief Model, i.e., perceived susceptibility (4 items), perceived severity ( 5 items), perceived benefits ( 4 items), perceived barriers (4 items), cues to action (4 items), general health motivation (4 items), and self-efficacy ( 2 items). All items are set up as statements in which participants answer on a 5-point Likert scale from "strongly disagree" (score 1) to "strongly agree" (score 5). The Health Belief Model and the MCLHB-DRR Scale are explained in more depth in the paper describing the development of the scale ${ }^{24}$.

The online follow-up questionnaire after the 12-weeks intervention period included the MCLHB-DRR Scale again, plus a custom questionnaire on user appreciation. Participants who reported not to have installed the app completed statements on reasons why, and participants who reported to have installed and used the app completed statements on appreciation and perceived knowledge gain, accompanying illustrative screenshots of the app. Users of the extended app version answered extra statements evaluating the additional features of this app. In answering the statements, participants were asked to what extent they agreed or disagreed on a 5-point Likert scale ranging from "strongly agree" to "strongly disagree". The statements of the active app users are displayed in Figure 4 of the Results Section. This questionnaire was developed in consultation with the department of Health Promotion of Maastricht University, based on other proof-of-concept studies, and tested on understandability in two pilot studies in responders from the same target population.

This study used the 23-item Dutch validated version of the MCLHB-DRR as a primary outcome, in which item 4 (perceived susceptibility), 10 and 13 (perceived benefits) and 25 (general health motivation) from the original scale were deleted because of low factor loadings ${ }^{25}$. In order to perform 
sensitivity analyses, the four deleted items of the Dutch validated scale were included in the assessment and the original 27-item version was also calculated.

The MijnBreincoach mobile app (standard version)

\section{Key functionalities}

The standard app version was designed to give people insight into their personal dementia risk profile and to identify room-for-improvement, based on the validated LIfestyle for BRAin health (LIBRA) score ${ }^{26}$. The LIBRA score, that was already used as a framework for a dementia risk reduction intervention discussed in a recent review, consists of 12 modifiable risk and protective factors for cognitive decline and dementia and has been extensively shown to predict dementia risk $8,27,28$ and change in multimodal lifestyle interventions ${ }^{29,30}$. Multimedia Appendix 2 provides an overview of all LIBRA factors and the instruments and cut-offs used. Figure 2 gives an overview of the different functionalities within the app and Multimedia Appendix 3 provides accompanying illustrative screenshots of the user interface. First, users completed a comprehensive assessment of the 12 LIBRA factors (see step 1 in Figure 2, and Multimedia Appendix $3^{a}$ ). After completion, a personal profile was created (See step 2 in Figure 2, and Multimedia Appendix $3^{\text {b) }}$ that gave people insight into identified areas of healthy behaviour (to promote maintenance), identified areas of unhealthy behaviour (to promote change) and identified chronic vascular/metabolic conditions (to promote appropriate management). Next, users were invited to choose a lifestyle topic or health condition of interest to work on (step 3 in Figure 2). They then received a short daily notification (the "Walnut of the day"; 14 in total) which contained a short text message providing either educational information, a brain-health advice, a quiz item, or a behavioural challenge in a pseudo-random fashion (See steps 4 and 5 in Figure 2, and Multimedia Appendix $3^{\mathrm{b}, \mathrm{c}}$ ). If the daily notification was not opened, its content was presented again the next day. Users could change their topic of interest at any time (step 6 in Figure 2). 


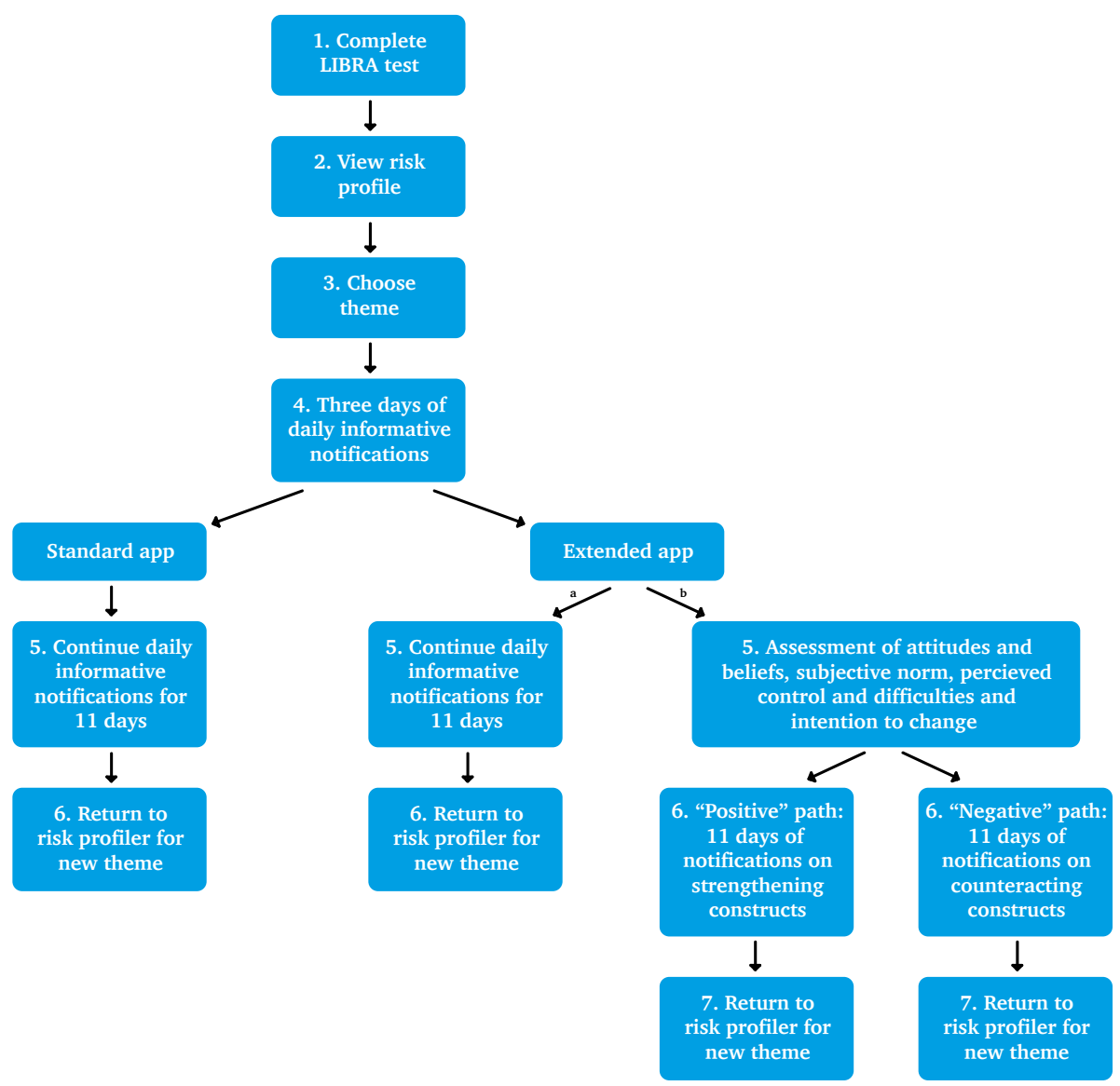

Figure 2. Graphic overview of the functionalities of the standard and extended MijnBreincoach

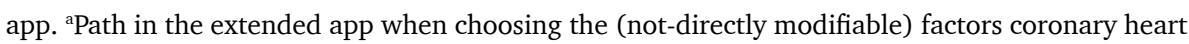
disease, chronic kidney disease, diabetes, cholesterol, hypertension, obesity or depression. ${ }^{\text {bPath }}$ in the extended app when choosing the (directly modifiable) factors alcohol consumption, physical inactivity, smoking, diet or cognitive activity. LIBRA: LIfestyle for BRAin health index. 
Features of the extended app

Key functionalities

The additional aim of the extended version of the app was to guide participants further in the process of behaviour change, by addressing beliefs and attitudes about lifestyle and health behaviour changes for dementia risk reduction. This additional feature was only integrated for the five LIBRA factors that were directly modifiable through individual lifestyle change (i.e., healthy diet, physical activity, cognitive activity, smoking, alcohol use; see step 5b of Figure 2). The Determinants of Lifestyle Behavior Questionnaire (DLBQ) ${ }^{31}$, an instrument based on Ajzen's Theory of Planned Behavior ${ }^{32}$, was used to assess the determinants attitudes, subjective norms, perceived control and difficulties and intention to change for physical activity, dietary behaviour and smoking and to provide tailored advice. Similar items were constructed to also assess determinants of the LIBRA factors "cognitive activity" and "alcohol use" (e.g., perceived difficulties: "I find it difficult not to smoke under stressful circumstances" was changed into "I find it difficult to drink less alcohol under stressful circumstances"). The daily notifications for the other seven LIBRA factors were the same as the standard version of the app (see step 5 a of Figure 2). Based on a custom-made cut-off score, users were classified in a positive or negative path. In the next eleven days, participants received messages to counteract negative and strengthen positive constructs. $O$ The messages contained text, but also active elements, such as writing down (dis) advantages of changing behaviour, goals and action plans to prevent relapse to old behaviour. Similar to the standard app, each topic consisted of 14 daily notifications. In addition, the app was extended with interactive functions, based on user feedback from the 2018-study. First, the layout of the administration of the LIBRA assessment was changed from a traditional questionnaire-design to a chat-interface design (with predetermined answering options) in order to make it more interactive. The last additional feature concerned the ability to re-read all daily notifications in one's personal profile, whereas participants from the standard version could read the short text messages only once they appeared.

\section{Development and testing of the apps}

In the developmental phase of both versions of the app, scrum sessions were organized with the software partners, important stakeholders and end-users in which the framework of the app was developed. The daily notifications 
were custom-made and derived from knowledge and advice from websites and flyers of evidence-based resources (e.g., the Dutch Heart Foundation, the Netherlands Nutrition Centre, Dutch Brain Foundation). After the research team had created 14 short notification messages based on these resources for each LIBRA theme, all content was checked by content experts on accuracy, completeness and readability. Further, we tested the acceptance and lookand-feel of both versions of the app in a client panel of the Alzheimer Centre Limburg of people with dementia, their caregivers and people with a specific interest in the topic of brain health and dementia. A final pilot study $(n=20)$ was conducted in which participants (from the target population) tested the apps for two consecutive weeks, to identify technical issues and survey items that needed further modification.

\section{Statistical analyses}

For the purpose of this study, the 2018- and 2019-study were combined in one sample in the analyses, while additional analyses stratified for study year and version of the app. For analyses on usage pattern of the app, we included data from the usage-tracking system of all participants that downloaded and used the app (see Figure 1, app usage). Analyses on reasons for non-use or dropout, appreciation of the app and beliefs and attitudes related to behavioural change included all that responded to the follow-up survey (see Figure 1, Baseline and Follow-up response). Due to incomplete follow-up responses, the number of people included in the analyses may differ from the number of people displayed in Figure 1.

Chi-squared tests and t-tests were used to investigate socio-demographics (age, sex, educational level), app characteristics (standard or extended version, operation system) and app usage in 1) participants lost-to-follow-up/ non-responders vs. study completers; 2) 2018 vs. 2019-study; 3) standard vs. extended version of the app; and 4) evaluation questions. To investigate change from baseline to post-intervention in the (Dutch validated, 23-item) MCLHBDRR Scale, we used linear mixed models adjusted for age, sex and educational level. The models included a random-intercept only, since likelihood ratio testing suggested the best fit for this approach. Use of linear mixed models allowed us to follow an intention-to-treat principle with maximum likelihood estimation ("imputation") of missing outcome data ${ }^{33}$. Then analyses were restricted to participants who completed subsequent steps in the app, using usage-tracking data as filters. Interaction terms between time ( $1=$ pre, $2=$ post $)$ 
and subsequently sex, level of education, age, year of study, version of the app and LIBRA profile were tested, followed by stratified analyses in case of statistical significance $(P<0.05)$. Analyses on the MCLHB-DRR Scale were repeated in a sensitivity analysis using the original 27 -item scale. In another sensitivity analysis, participants from the 2019-sample who were recruited via the extra recruitment strategies were excluded to investigate whether the results were driven by this difference in recruitment. All analyses were performed in Stata 13.1 (StataCorp, College Station, TX, USA), and the level of statistical significance was $p<0.05$ in two-tailed tests. 


\section{Results}

\section{Participants}

As shown in Figure 1 and Multimedia Appendix 1, a total of 686 individuals were invited to participate, of whom 299 (response rate: 43.6\%) completed the baseline survey and subsequently received information on how to install the app. The app was used by 167 (55.9\%) participants. In total, 190 (63.6\%) participants completed the follow-up questionnaire, of which 70 (36.8\%) did not use the app and answered questions on reasons for non-use and 120 $(63.2 \%)$ used the app and answered questions on user appreciation. A loss to follow-up analysis on survey response revealed no differences between studycompleters and dropouts/non-responders for sex $\left(\chi^{2}(1)=0.027, P=.87\right)$, age $(\mathrm{t}(297)=-0.664, P=.51)$, level of education $\left(\chi^{2}(1)=0.202, P=.65\right)$, risk factor profile $(\mathrm{t}(161)=1.50, P=.14)$, version of app (standard vs. extended version; $\chi^{2}(1)=2.19, P=.14$ ) or operating system (iOS vs. Android; $\chi^{2}(1)=3.143$, $P=.08)$. There were no socio-demographic differences observed in version of the app and year of study, except for a higher percentage of high-educated individuals participating in the 2019-study compared to the 2018-study. Table 1 describes the characteristics of the total study sample, and stratified by year of study. 
Table 1. Characteristics of the total sample and stratified by study year.

\begin{tabular}{|c|c|c|c|c|}
\hline Variables $^{\mathrm{a}, \mathrm{b}}$ & $\begin{array}{r}\text { Total baseline } \\
\text { sample }(n=299)\end{array}$ & 2018-study $(n=89)$ & $\begin{array}{r}2019 \text {-study } \\
(n=210)\end{array}$ & $P$ \\
\hline $\begin{array}{l}\text { Version of the } \\
\text { app, n (\%) }\end{array}$ & & & & $<.001$ \\
\hline Standard version & $198(66.2 \%)$ & $89(100 \%)$ & $109(51.9 \%)$ & \\
\hline Extended version & $101(33.8 \%)$ & NA & $101(48.1 \%)$ & \\
\hline Sex, n (\%) & & & & .55 \\
\hline Female & $149(49.8 \%)$ & $42(47 \%)$ & $107(51.0 \%)$ & \\
\hline Male & $150(50.2 \%)$ & $47(53 \%)$ & $103(49.1 \%)$ & \\
\hline $\begin{array}{l}\text { Age, mean } \pm \text { SD } \\
\text { (range) }\end{array}$ & $60.2 \pm 8.3(40-74)$ & $61.4 \pm 7.8(40-74)$ & $59.7 \pm 8.5(41-74)$ & .11 \\
\hline $\begin{array}{l}\text { Age groups, } n \\
(\%)\end{array}$ & & & & .09 \\
\hline $40-59$ years & $133(44.5 \%)$ & $33(37 \%)$ & $100(47.6 \%)$ & \\
\hline $60-75$ years & $166(55.5 \%)$ & $56(63 \%)$ & $110(52.4 \%)$ & \\
\hline $\begin{array}{l}\text { Level of } \\
\text { education }{ }^{\mathrm{c}}, \mathbf{n}(\%)\end{array}$ & & & & .03 \\
\hline Low & $19(6 \%)$ & $10(11 \%)$ & $9(4 \%)$ & \\
\hline Middle & $104(34.8 \%)$ & $35(39 \%)$ & $69(33 \%)$ & \\
\hline High & $176(58.9 \%)$ & $44(49 \%)$ & $132(62.9 \%)$ & \\
\hline $\begin{array}{l}\text { Marital status, } \\
\text { n (\%) }\end{array}$ & & & & .91 \\
\hline $\begin{array}{l}\text { Married/living } \\
\text { together }\end{array}$ & $241(80.6 \%)$ & $73(82 \%)$ & $168(80.0 \%)$ & \\
\hline Unmarried & $14(5 \%)$ & $3(3 \%)$ & $11(5 \%)$ & \\
\hline Divorced & $33(11 \%)$ & $10(11 \%)$ & $23(11 \%)$ & \\
\hline Widowed & $11(4 \%)$ & $3(3 \%)$ & $8(4 \%)$ & \\
\hline
\end{tabular}




\begin{tabular}{|c|c|c|c|c|}
\hline $\begin{array}{l}\text { App usage in } \\
\text { general, n (\%) }\end{array}$ & & & & .49 \\
\hline Daily & $276(92.3 \%)$ & $81(91 \%)$ & $195(92.9 \%)$ & \\
\hline Weekly & $13(4 \%)$ & $4(5 \%)$ & $9(4 \%)$ & \\
\hline Monthly & $2(0.7 \%)$ & 0 & $2(1 \%)$ & \\
\hline Never & $8(3 \%)$ & $4(5 \%)$ & $4(2 \%)$ & \\
\hline
\end{tabular}

a Percentages do not always add up to $100 \%$ because of rounding. ${ }^{\text {b }}$ Abbreviations: NA: not applicable; SD: standard deviation. ' Self-reported highest finalized degree, divided into low (primary school or low vocational education), middle (intermediate secondary education or intermediate vocational or higher secondary education) and high (higher vocational education or university). 
Usage tracking data: usage pattern

Of all participants $(n=299), 167(55.9 \%)$ installed the app according to the usage tracking data. Approximately $19 \%$ of the total sample contacted the research team via email or telephone with questions on installing the app and password resets. Women $(\mathrm{n}=94,63 \%)$ installed the app more often than men did $\left(n=73,49 \% ; \chi^{2}(1)=6.304, P=.01\right)$. The usage of the app was on average 30.4 days (from installation to last daily notification; SD 39.9, range $1-181$, with $40 \%(n=66)$ of the participants using the app less than seven days). Participants received on average 20.3 (SD 23.1; range $1-98$ ) daily notifications, with $41 \%(\mathrm{n}=68)$ receiving six or less and $7 \%(\mathrm{n}=11)$ receiving more than 70 notifications.

Of all participants who were randomized to the extended version of the app ( $\mathrm{n}=101), 50$ (49.5\%) actually installed the app but only 16 of these 50 participants (32\%) used the main additional feature of the extended version (tailoring based on the DLBQ constructs). Those who did not use this additional feature $(n=34)$ either chose other themes $(n=13 ; 38 \%)$ or did not receive more than three daily notifications (in order for tailoring to appear; $n=21$; $62 \%)$.

Figure 3 displays the frequency of the subsequent steps/functions used in the app and the themes chosen to receive daily notifications. Among the 167 participants who installed the app, the majority $(n=163,97.6 \%)$ viewed the personal risk profile. Health factors that were most often present were hypertension $(\mathrm{n}=81,50 \%)$, high cholesterol $(\mathrm{n}=52,32 \%)$ and chronic kidney disease $(n=36,22 \%)$. Room for lifestyle improvement was highest for physical activity ( $\mathrm{n}=137,84.1 \%)$, healthy diet $(\mathrm{n}=134,82.2 \%)$ and cognitive activity $(n=69,42 \%)$. These were also the lifestyle themes that were most often chosen to receive daily notifications (cognitive activity $n=65,40 \%$, healthy diet $n=63$, $39 \%$ and physical activity $n=60,37 \%$ ). No differences in app usage was found between users of different versions of the app and year of study. 


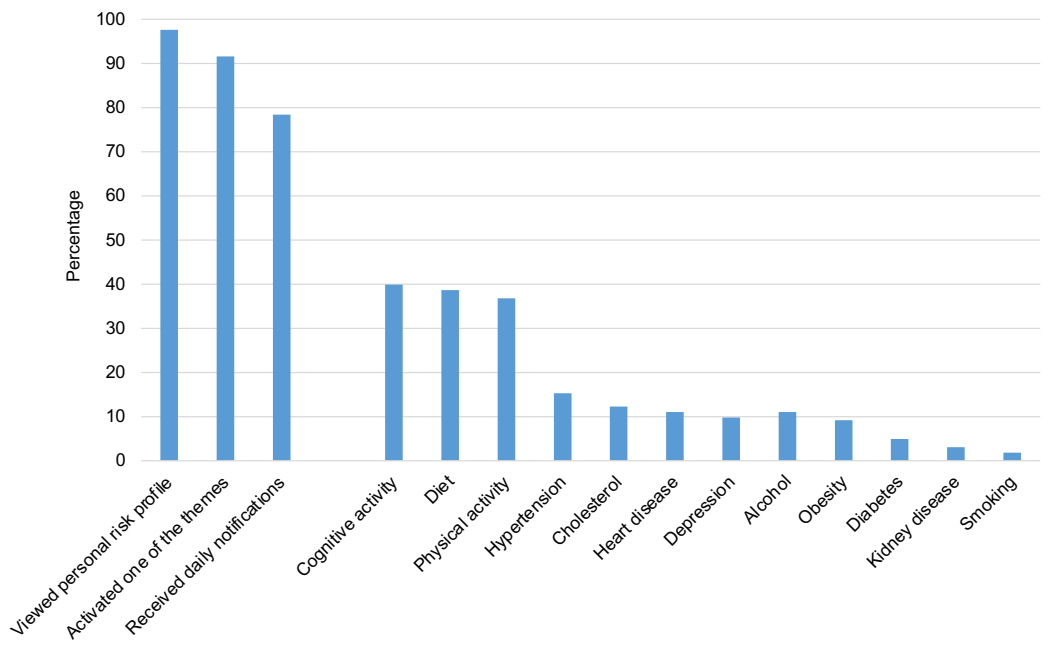

Figure 3. Subsequent steps taken in the app according to user-tracking data. Individual factors reflect the percentage of participants that have activated this theme in the app to receive daily notifications (information, quiz item, behavioural challenge).

\section{Online questionnaires}

Reasons for non-use and dropout

Of all 70 participants that did not use the app and responded to the followup assessment, 41 completed items on reasons for non-use (the remaining 29 had incomplete survey responses). Of all participants that did use the app and responded to the follow-up assessment $(n=120), 43(36 \%)$ reported to have stopped using the app during the intervention. Reasons for non-use and dropout were mostly technical problems such as problems with installation, sporadic log-outs and forgetting username or password $(\mathrm{n}=21 ; 51 \%$ and $\mathrm{n}=30 ; 70 \%$ respectively), followed by motivational issues $(\mathrm{n}=11 ; 27 \%$ and $n=5 ; 12 \%)$ and lack of time $(n=9 ; 22 \%$ and $n=8 ; 19 \%)$. No differences were observed in reasons for non-use and dropout for age, sex and educational level between the two app versions (Table 2). 
Table 2. Reasons for non-use and dropout during the study by version of the app.

\begin{tabular}{lrrrr}
\hline & \multicolumn{3}{c}{ Non-use (n=41) } & Dropout (n=43) \\
\hline $\begin{array}{l}\text { Self-reported } \\
\text { reasons, } \mathbf{n}(\%)\end{array}$ & $\begin{array}{r}\text { Standard version } \\
(n=22)\end{array}$ & $\begin{array}{r}\text { Extended version } \\
(n=19)\end{array}$ & $\begin{array}{r}\text { Standard version } \\
(n=22)\end{array}$ & $\begin{array}{r}\text { Extended version } \\
(n=21)\end{array}$ \\
$\begin{array}{l}\text { Motivational } \\
\text { issues }\end{array}$ & $6(27 \%)$ & $5(26 \%)$ & $1(5 \%)$ & $4(19 \%)$ \\
Lack of time & $6(27 \%)$ & $3(16 \%)$ & $5(23 \%)$ & $3(14 \%)$ \\
$\begin{array}{l}\text { Technical } \\
\text { problems }\end{array}$ & $10(46 \%)$ & $11(58 \%)$ & $16(73 \%)$ & $14(67 \%)$ \\
\hline
\end{tabular}

Appreciation of the app and perceived knowledge gain

Of all participants that used the app and responded to the follow-up assessment $(n=120), 21$ stopped before or during the user evaluation, leaving 99 to 106 participants in these analyses, depending on the item. Participants reported to have spent an average of 6.4 minutes per day on the app (SD 7.8, range $0-45$ ). The app was overall graded with a 6.3 (SD 2.0; range $2-10$ ) on a scale of 0 to 10, with higher scores being better. Low-to-middle educated participants spent more minutes per day $(9.4, \mathrm{n}=35)$ on the app than high educated participants $(4.7, \mathrm{n}=65)$ did $(\mathrm{t}(98)=2.97, P=.004)$, and also graded the app somewhat higher (low/middle: 6.8, $\mathrm{n}=34$; high: 6.0, $\mathrm{n}=65$; $\mathrm{t}(97)=$ $2.06, P=.04) .66 \%(n=67)$ of all participants expressed a desire to keep using the app. No differences were observed for sex, age, study year and version of the app.

Figure 4 shows the results of the self-reported data of the post-assessment survey concerning the user evaluation. More than three quarters of the sample $(n=102)$ found the app enjoyable and stated that the app provided a positive approach towards brain health. The vast majority stated that the daily notifications were easy to read. With regard to the perceived knowledge gain, more than $80 \%$ stated to have a better understanding of the concept of brain health, that the app made them think of their own brain health and that they were able to use the given advices. No differences were observed 
between years of study and versions of the app, except that participants using the extended version less often stated that the app was enjoyable $(63 \%, \mathrm{n}=22$ vs. $82 \%, n=55 ; \chi^{2}(1)=4.60, P=.03$ ) compared to participants using the standard version. Differences in age, sex and education level were observed. Low-to-middle educated participants stated more often than higher educated participants that the daily notifications were easy to read $(100 \%, n=36$ vs. $\left.89 \%, \mathrm{n}=59 ; \chi^{2}(1)=4.10, P=.04\right)$, that they perceived the advices as useful $\left(100 \%, \mathrm{n}=36\right.$ vs. $\left.77 \%, \mathrm{n}=51 ; \chi^{2}(1)=9.59, P=.002\right)$, and have gained a better understanding of brain health ( $94 \%, \mathrm{n}=33$ vs. $\left.74 \%, \mathrm{n}=49, \chi^{2}(1)=6.02, P=.01\right)$. The average age was younger in those agreeing (or answering neutral) to the statements that the daily notifications were easy to read $(n=102$; average age "agree/neutral": 59 years; "disagree": 66 years; $\mathrm{t}(100)=-2.30, P=.02)$ and that the app was easy to use $(\mathrm{n}=102$; agree/neutral: 58 years, disagree: 63 years; $\mathrm{t}(100)=-2.77, P=.01)$. 


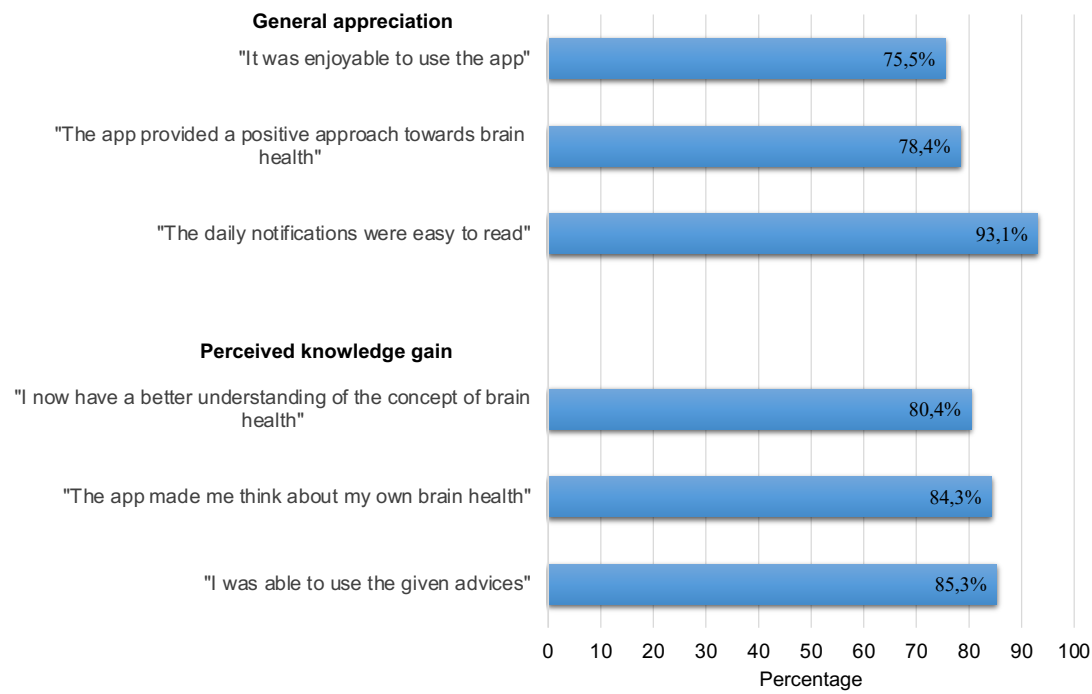

Figure 4. User appreciation of the MijnBreincoach app ( $n=102)$. Percentages reflect the amount of participants agreeing with the statement or answering neutrally (totally agree, agree, and neither agree nor disagree).

Beliefs and attitudes related towards behavioural change: baseline differences $(n=299)$

At baseline, women scored higher on general health motivation compared to men $(\mathrm{t}(297)=-1.97, P=.05)$. Low-to-middle educated participants scored higher at baseline on perceived severity $(\mathrm{t}(297)=2.35, p=.019)$ and perceived barriers $(\mathrm{t}(297)=2.48, P=.014)$, compared to high educated participants. Participants under the age of 60 years scored higher at baseline on perceived benefits towards changing lifestyle and health behaviour to reduce dementia risk $(\mathrm{t}(297)=2.71, P=.01)$ compared to participants aged 60 or above. No baseline differences were observed for users of the standard versus extended version of the app, and for the 2018- versus 2019-sample.

Beliefs and attitudes related towards behavioural change: main effects In the intention-to-treat analyses, no change was found on the constructs in the total sample (see Table 3). Interaction analyses suggested that perceived benefits decreased over time in women $(B=-0.343$, CI $-0.630--0.057, P=.02)$ and in participants from the 2019-study $(B=-0.270, C I-0.498-0.042, P=.02)$, 
while there was a non-significant increase in perceived benefits in participants of the 2018-study ( $B=0.404$, CI $-0.025-0.834, P=.07$ ). When restricting analyses to participants that had actively used the app, the perceived benefits $(\mathrm{B}=0.615$, $\mathrm{CI} 0.115-1.115, P=.02)$ and cues to action $(\mathrm{B}=1.170$, $\mathrm{CI} 0.358$ - 1.981, $P=.01$ ) increased significantly in the 2018 sample, but not in the 2019 sample. Perceived barriers further increased in participants that used the standard version of the app ( $B=0.588$, CI $0.189-0.987, P=.004)$, but this became non-significant when restricting analyses to active users $(B=0.414, \mathrm{CI}$ $-0.079-0.908, P=.10)$. A non-significant decrease in perceived barriers was observed in the 2019-study, both in the total sample $(B=-0.370$, CI $-0.899-$ $0.158, P=.17)$ and in those who actively used the app (B=-0.373, CI -1.070 $-0.324, P=.30$ ). 
Table 3. Pre-post comparison of beliefs and attitudes towards behavioural change.

\begin{tabular}{lrccc}
\hline $\begin{array}{l}\text { Variables } \\
\text { Total pre-survey, } \\
\text { mean } \pm \text { SD; range }\end{array}$ & $\begin{array}{c}\text { Total post-survey, } \\
\text { mean } \pm \text { SD; range }\end{array}$ & B & $\boldsymbol{P}$ \\
\hline Perceived susceptibility & $8.9 \pm 2.0 ; 3-14$ & $8.8 \pm 2.2 ; 3-15$ & -0.07 & 0.56 \\
Perceived severity & $15.6 \pm 3.3 ; 5-24$ & $15.5 \pm 3.5 ; 5-25$ & -0.26 & 0.18 \\
Perceived benefits & $7.1 \pm 1.4 ; 3-10$ & $7.0 \pm 1.4 ; 3-10$ & -0.13 & 0.20 \\
Perceived barriers & $8.3 \pm 2.5 ; 4-16$ & $8.4 \pm 2.5 ; 4-15$ & 0.23 & 0.16 \\
Cues to action & $11.9 \pm 2.6 ; 4-20$ & $11.9 \pm 2.6 ; 4-18$ & 0.09 & 0.60 \\
General health motivation & $12.3 \pm 1.7 ; 7-15$ & $12.3 \pm 1.7 ; 7-15$ & 0.01 & 0.92 \\
Self-efficacy & $6.6 \pm 1.4 ; 2-10$ & $6.5 \pm 1.4 ; 2-10$ & -0.05 & 0.62 \\
\hline
\end{tabular}

${ }^{\text {a }}$ Analyses adjusted for educational level, sex and age.

\section{Sensitivity analysis}

Sensitivity analyses using the original 27-item scale of the MCLHB-DRR showed similar results. For perceived benefits, there was no longer a significant interaction term for sex $(B=-0.245$, $C I-0.830-0.341, P=.41)$ and year of study $(B=-0.486$, $C I-1.196-0.224, P=.18)$ with time. Results on usage pattern and the self-reported questionnaires were similar when excluding those from the 2019-sample who were recruited via the extra recruitment strategies. 


\section{Discussion}

\section{Principal findings and interpretation}

This proof-of-concept study using a pre-post design investigated the MijnBreincoach mobile application on usability, acceptability, knowledge and beliefs and attitudes related to motivation to change behaviour for dementia risk reduction in 299 non-demented, community-dwelling Dutch participants in midlife. The app was evaluated positively, with most participants stating to have improved their knowledge on brain health. Sub-group analyses showed that women, younger $(<60 y)$ and low-to-middle educated participants evaluated the app most positive. Very few participants used the additional features of the extended version of the app. No or only modest pre-post changes were observed on beliefs and attitudes related to dementia risk reduction.

It seemed that the app covered the topic of dementia risk reduction in an appealing manner, and was successful in transferring knowledge to the general public. This finding is in line with previous web-based studies evaluating the usefulness of providing online information ${ }^{34}$, also specifically for dementia risk reduction ${ }^{35-37}$. However, beliefs and attitudes towards dementia risk reduction did not change or only changed in an inconsistent pattern in subanalyses of participants that actively used the app. Given the observation that $40 \%$ of all participants received six or less daily notifications and that the effect measurement only took place after 12 weeks, one explanation could be that beliefs and attitudes did change directly after viewing one's risk profile and/ or receiving the daily messages, but that this effect disappeared over time. It should also be noted that our sample was recruited based on their interest in the topic of brain health and mainly consisted of middle-to high educated participants who had a low risk profile (i.e., low average LIBRA scores compared to population-based studies investigating LIBRA ${ }^{8,27,38}$ ). It might thus be that the baseline measurement of beliefs and attitudes was a true reflection of one's risk perception, which could have been a barrier to find changes over time. Other explanations concern the scale we used to assess beliefs and attitudes towards dementia risk reduction. It could be that the MCLHB-DRR scale is particularly suitable to identify individuals or subgroups that would benefit most of an intervention and then tailor interventions towards their specific beliefs and needs ${ }^{24}$, and that the scale is less suitable as an outcome measure in a mHealth-only lifestyle intervention of three months. The scale has, to our knowledge, not been used to assess the effect of (lifestyle) interventions 
and, therefore, comparisons with other studies cannot be made. Last, we encountered some technical issues that could have affected the user adherence and experience. In the week after the post-assessments were e-mailed during the 2019-study, Maastricht University was hit by an institution-wide cyberattack. Because of this, our survey program was no longer online, and we were unable to reach participants. This resulted in a gap in time of five weeks between the end of the intervention period and the effect measurement, which could have influenced the results. Furthermore, there were technical issues regarding the app, involving registration, login and server time-outs. In fact, $44 \%$ of our sample did not even install the app, and technical issues were the most frequent self-reported reason for non-use or dropout, which is a common problem in web-based lifestyle programs for brain health ${ }^{22}$. To illustrate, of participants that installed the app and activated a LIBRA theme, $14.4 \%$ (see Figure 3) never received a daily notification, which is likely the consequence of system failure log-outs. As for the 2019 sample specifically, stronger rules on privacy assurance resulted in extra steps to download the extended app, which resulted in high dropout rates.

Women had a higher baseline general health motivation and used the app more often than men, which is in line with earlier studies investigating webbased lifestyle interventions ${ }^{19,39}$. Younger participants rated the readability of the daily notifications more positively, and found the app more enjoyable, compared to older participants. Several studies show that older participants of web-based interventions may experience barriers ${ }^{21,}{ }^{40}$. However, older participants still evaluated the app in a positive manner, and there were no differences between age groups in use of the app or dropout of the study. The finding that low-to-middle educated participants spent more time on the app could suggest that they were more engaged. Low-to-middle educated participants also gave the app a slightly better overall appreciation. Importantly, the app was designed to make it suitable for less educated or less health literate people (e.g., prompting people with short messages that appeared automatically), since these sub-groups are often hard-to-reach in web-based lifestyle interventions ${ }^{19,39}$. Previous research showed that lower education and lower socio-economic position is associated with a higher risk for dementia, and that this is substantially explained by an unfavourable modifiable risk score ${ }^{28}$. Engaging this hard-to-reach group is thus important for achieving the goals of dementia risk reduction in the general population and reducing brainhealth inequalities ${ }^{10}$. 


\section{Strengths and limitations}

Strengths of this study include the extensive pre-post measurements, including both self-reported data, and objective usage-tracking. Also, we tested the accuracy, completeness and readability of the custom-made daily notifications by renowned experts in the field, and tested the general acceptance of this app in potential end-users and a client panel. In addition, we designed the app around multiple dementia risk factors (12 in total) and collaborated with different stakeholders in a multidisciplinary approach, which is in line with WHO guidelines ${ }^{1}$. Next, in contrast to most other web-based interventions to improve brain health ${ }^{22}$, our mHealth platform is free of charge available to the general public in the Netherlands and could easily be adapted to the setting and language of other countries and could therefore provide a bridge between dementia risk reduction research and the community. This study, however, also has limitations that need to be addressed. First, this study did not use a control group and randomization (in the total sample), which means that the internal validity of the intervention is low. Furthermore, recruitment bias may have occurred due to the inclusion from responders of a previous dementia risk awareness survey ${ }^{23}$ who expressed interest in brain health studies, and additional recruitment via the websites of the Dutch Brain Foundation and a large health care institution. This makes our results less generalizable to the intended target group of the general population. Yet, sensitivity analyses excluding participants of the extra recruitment strategies did not change results.

Next, this study was limited by the considerable amount of participants that did not use the app $(n=132,44.2 \%)$ and/or did not respond to the follow-up assessment $(n=109,36.5 \%)$. This should be taken into consideration when interpreting the results. It most likely means that the app was only used by participants who were (a priori) highly motivated to participate. To generalize these findings to the general public, usage rates should be improved. Still, systematic reviews show that attrition rates are often high in digital interventions and our loss to follow-up was equal to the reported mean loss to follow-up reported in one of the reviews of $47 \%{ }^{41,42}$. Furthermore, except for the export of the usage pattern of participants, all outcome measures were based on selfreport. Socially desirable answers should therefore be taken into account when interpreting results on appreciation of the app and perceived knowledge gain. Last, all communication and instructions to use the app were given via e-mail. In retrospect, it may have been more beneficial to organize dedicated meetings 
(online or at the research centre), in which participants were guided in how to install and use the app, or to provide participants with devices that already had the app preloaded. This might have solved some of the technical issues that participants encountered and might also have increased active involvement.

\section{Implications and recommendations future research}

This study was designed as a proof-of-concept, aimed at identifying both the barriers and difficulties in the development of a mHealth tool for dementia risk reduction, and the advantages and gains, in order to improve our understanding of important factors that need to be considered in future studies. First of all, it is important to improve the usability of the tool before executing further intervention studies, since it is impossible to draw conclusions concerning the effectiveness of (parts of) an intervention if participants were not able to use it the way it was supposed to. In order to improve usability, the technical issues must be resolved and the app needs to be further developed (e.g., profile options to set the preferred amount of daily notifications). When further developing the behavioural change aspects of this app, the right balance between of eHealth and mHealth, (e.g., scalable to implement populationwide, easy to implement in daily living), and in-person counselling (e.g., personalized care, motivational interviewing techniques) should be kept in mind ${ }^{43}$. For example, a blended care approach, in which the mHealth tool is combined with face-to-face or online coaching sessions, could have beneficial effects on lifestyle improvements ${ }^{44,45}$. The extended version of this app did attempt to tailor the daily notifications towards the individual, but still was a stand-alone intervention without behavioural support of a coach. Next, in retrospect, including items on literacy of dementia risk reduction would have strengthened the evidence from the self-reported evaluation of the app that, despite the fact that motivation to change behaviour did not improve, the app was successful in increasing participants' knowledge on dementia risk reduction. Last, future studies should consider randomized controlled designs in investigating the effect of mobile applications to improve brain health in order to answer questions regarding causality. 


\section{Conclusions}

This study suggests that the mHealth intervention MijnBreincoach could be a useful contribution to reach the potential of primary prevention of dementia, by improving knowledge on the subject in an appealing manner. Yet, improving the use (i.e., resolve technological issues, expand functions of the app) and investigating the effect of extended behavioural change aspects is important to take into consideration in future studies. 


\section{References}

1. Risk reduction of cognitive decline and dementia: WHO guidelines. 2019, World Health Organization: Geneva.

2. Norton $S$, Matthews FE, Barnes DE, Yaffe K, Brayne C. Potential for primary prevention of Alzheimer's disease: an analysis of population-based data. Lancet Neurol 2014;13:788-94.

3. Livingston G, Huntley J, Sommerlad A, et al. Dementia prevention, intervention, and care: 2020 report of the Lancet Commission. Lancet 2020;396:413-46.

4. Livingston $\mathrm{G}$, Sommerlad A, Orgeta V, et al. Dementia prevention, intervention, and care. Lancet 2017;390:2673-734.

5. Baumgart M, Snyder HM, Carrillo MC, Fazio S, Kim H, Johns H. Summary of the evidence on modifiable risk factors for cognitive decline and dementia: A population-based perspective. Alzheimers Dement 2015;11:718-26.

6. Yu JT, Xu W, Tan CC, et al. Evidencebased prevention of Alzheimer's disease: systematic review and meta-analysis of 243 observational prospective studies and 153 randomised controlled trials. J Neurol Neurosurg Psychiatry 2020;91:1201-09.

7. Kivipelto M, Mangialasche F, Ngandu T. Lifestyle interventions to prevent cognitive impairment, dementia and Alzheimer disease. Nature reviews. Neurology 2018;14:653-66.

8. Vos SJB, van Boxtel MPJ, Schiepers OJG, et al. Modifiable Risk Factors for Prevention of Dementia in Midlife, Late Life and the Oldest-Old: Validation of the LIBRA Index.
J Alzheimers Dis 2017;58:537-47.

9. Heger I, Deckers K, van Boxtel M, et al. Dementia awareness and risk perception in middle-aged and older individuals: baseline results of the MijnBreincoach survey on the association between lifestyle and brain health. BMC Public Health 2019;19:678.

10. Steyaert J, Deckers K, Smits C, et al. Putting primary prevention of dementia on everybody's agenda. Aging Ment Health 2020:1-5.

11. Barak Y, Gray AR, Rapsey C, Scott K. The Dunedin dementia risk awareness project: pilot study in older adults. Int Psychogeriatr 2020;32:241-54.

12. Cations M, Radisic G, Crotty M, Laver KE. What does the general public understand about prevention and treatment of dementia? A systematic review of population-based surveys. PLoS One 2018;13.

13. Parial LL, Lam SC, Ho JYS, Suen LKP, Leung AYM. Public knowledge of the influence of modifiable cardiovascular risk factors on dementia: a systematic literature review and meta-analysis. Aging Ment Health 2020;7:1-15.

14. World Alzheimer Report 2019. Attitudes to Dementia. 2019, Alzheimer's Disease International: London.

15. Orgeta V, Mukadam N, Sommerlad A, Livingston G. The Lancet Commission on Dementia Prevention, Intervention, and Care: a call for action. Ir $J$ Psychol Med 2019;36:85-88. 
16. Low LF, Anstey KJ. Dementia literacy: recognition and beliefs on dementia of the Australian public. Alzheimers Dement 2009;5:43-9.

17. StatLine Centraal Bureau voor de Statistiek (CBS). Internet; toegang, gebruik en faciliteiten; 2012-2019. 2019. https://opendata.cbs.nl/\#/CBS/nl/ dataset/83429NED/table? $\mathrm{dl}=2 \mathrm{~F} 8 \mathrm{AA}$ (May 27, 2020, date last accessed).

18. Saner $H$, van der Velde E. eHealth in cardiovascular medicine: A clinical update. Eur J Prev Cardiol 2016;23:5-12.

19. Van 't Riet J, Crutzen $R$, De Vries $H$. Investigating predictors of visiting, using, and revisiting an online healthcommunication program: a longitudinal study. J Med Internet Res 2010;12:e37.

20. Chow CK, Ariyarathna N, Islam SM, Thiagalingam A, Redfern J. mHealth in Cardiovascular Health Care. Heart Lung Circ 2016;25:802-7.

21. Kampmeijer $R$, Pavlova $M$, Tambor $M$, Golinowska S, Groot W. The use of e-health and m-health tools in health promotion and primary prevention among older adults: a systematic literature review. BMC Health Serv Res 2016;16 Suppl 5:290.

22. Wesselman LM, Hooghiemstra AM, Schoonmade LJ, de Wit MC, van der Flier WM, Sikkes SA. Web-Based Multidomain Lifestyle Programs for Brain Health: Comprehensive Overview and Meta-Analysis. JMIR Ment Health 2019;6:e12104.

23. Heger I, Köhler $S$, van Boxtel $M$, et al. Raising awareness for dementia risk reduction through a public health campaign: a pre-post study. BMJ Open 2020;10:e041211.

24. Kim S, Sargent-Cox K, Cherbuin N, Anstey KJ. Development of the motivation to change lifestyle and health behaviours for dementia risk reduction scale. Dement Geriatr Cogn Dis Extra 2014;4:172-83.

25. Joxhorst T, Vrijsen J, Niebuur J, Smidt N. Cross-cultural validation of the motivation to change lifestyle and health behaviours for dementia risk reduction scale in the Dutch general population. BMC Public Health 2020;20:788.

26. Deckers K, van Boxtel MP, Schiepers OJ, et al. Target risk factors for dementia prevention: a systematic review and Delphi consensus study on the evidence from observational studies. Int $J$ Geriatr Psychiatry 2015;30:234-46.

27. Schiepers OJG, Kohler S, Deckers K, et al. Lifestyle for Brain Health (LIBRA): a new model for dementia prevention. Int $J$ Geriatr Psychiatry 2018;33:167-75.

28. Deckers $K$, Cadar D, van Boxtel MPJ, Verhey FRJ, Steptoe A, Kohler S. Modifiable Risk Factors Explain Socioeconomic Inequalities in Dementia Risk: Evidence from a Population-Based Prospective Cohort Study. J Alzheimers Dis 2019;71:549-57.

29. Coley N, Hoevenaar-Blom MP, van Dalen JW, et al. Dementia risk scores as surrogate outcomes for lifestyle-based multidomain prevention trials-rationale, preliminary evidence and challenges. Alzheimers Dement 2020.

30. Deckers K, Köhler S, Ngandu T, et al. Quantifying dementia prevention potential in the FINGER randomized controlled 
trial using the LIBRA prevention index. Alzheimers Dement 2021.

31. Lakerveld J, Bot SD, Chinapaw MJ, Knol DL, de Vet HC, Nijpels G. Measuring pathways towards a healthier lifestyle in the Hoorn Prevention Study: the Determinants of Lifestyle Behavior Questionnaire (DLBQ). Patient Educ Couns 2011;85:e53-8.

32. Godin G, Kok G. The theory of planned behavior: a review of its applications to health-related behaviors. AJHP 1996;11:87-98.

33. White IR, Horton NJ, Carpenter J, Pocock SJ. Strategy for intention to treat analysis in randomised trials with missing outcome data. BMJ 2011;342:d40.

34. Wantland DJ, Portillo CJ, Holzemer WL, Slaughter R, McGhee EM. The effectiveness of Web-based vs. non-Webbased interventions: a meta-analysis of behavioral change outcomes. $J$ Med Internet Res 2004;6:e40.

35. Farrow M. User perceptions of a dementia risk reduction website and its promotion of behavior change. JMIR research protocols 2013;2:e15-e15.

36. O'Connor E, Farrow M, Hatherly C. Randomized Comparison of Mobile and Web-Tools to Provide Dementia Risk Reduction Education: Use, Engagement and Participant Satisfaction. JMIR Ment Health 2014;1:e4.

37. Anstey KJ, Bahar-Fuchs A, Herath P, et al. Body brain life: A randomized controlled trial of an online dementia risk reduction intervention in middle-aged adults at risk of Alzheimer's disease. Alzheimers Dement 2015;1:72-80.
38. Deckers K, Nooyens A, van Boxtel M, Verhey F, Verschuren M, Kohler S. Gender and Educational Differences in the Association between Lifestyle and Cognitive Decline over 10 Years: The Doetinchem Cohort Study. J Alzheimers Dis 2018;70:S31-S41.

39. Brouwer $\mathrm{W}$, Oenema $\mathrm{A}$, Raat $\mathrm{H}$, et al Characteristics of visitors and revisitors to an Internet-delivered computer-tailored lifestyle intervention implemented for use by the general public. Health Educ Res 2010;25:585-95.

40. Wildenbos GA, Peute L, Jaspers M. Aging barriers influencing mobile health usability for older adults: A literature based framework (MOLD-US). Int J Med Inform 2018;114:66-75.

41. Gold N, Yau A, Rigby B, Dyke C, Remfry EA, Chadborn T. Effectiveness of Digital Interventions for Reducing Behavioral Risks of Cardiovascular Disease in Nonclinical Adult Populations: Systematic Review of Reviews. $J$ Med Internet Res 2021;23:e19688.

42. Mathieu E, McGeechan K, Barratt A, Herbert R. Internet-based randomized controlled trials: a systematic review. $J$ Am Med Inform Assoc 2013;20:568-76.

43. Ryan K, Dockray S, Linehan C. A systematic review of tailored eHealth interventions for weight loss. Digit Health 2019;5:2055207619826685.

44. Joiner KL, Nam S, Whittemore R. Lifestyle interventions based on the diabetes prevention program delivered via eHealth: A systematic review and meta-analysis. Prev Med 2017;100:194-207.

45. Kouwenhoven-Pasmooij TA, Robroek SJW, 
Chapter 6

Kraaijenhagen RA, et al. Effectiveness of the blended-care lifestyle intervention 'PerfectFit': a cluster randomised trial in employees at risk for cardiovascular diseases. BMC Public Health 2018;18:766. 


\section{Additional Files}

Additional File 1. Flowchart of the recruitment process, actual app use and survey response per study year

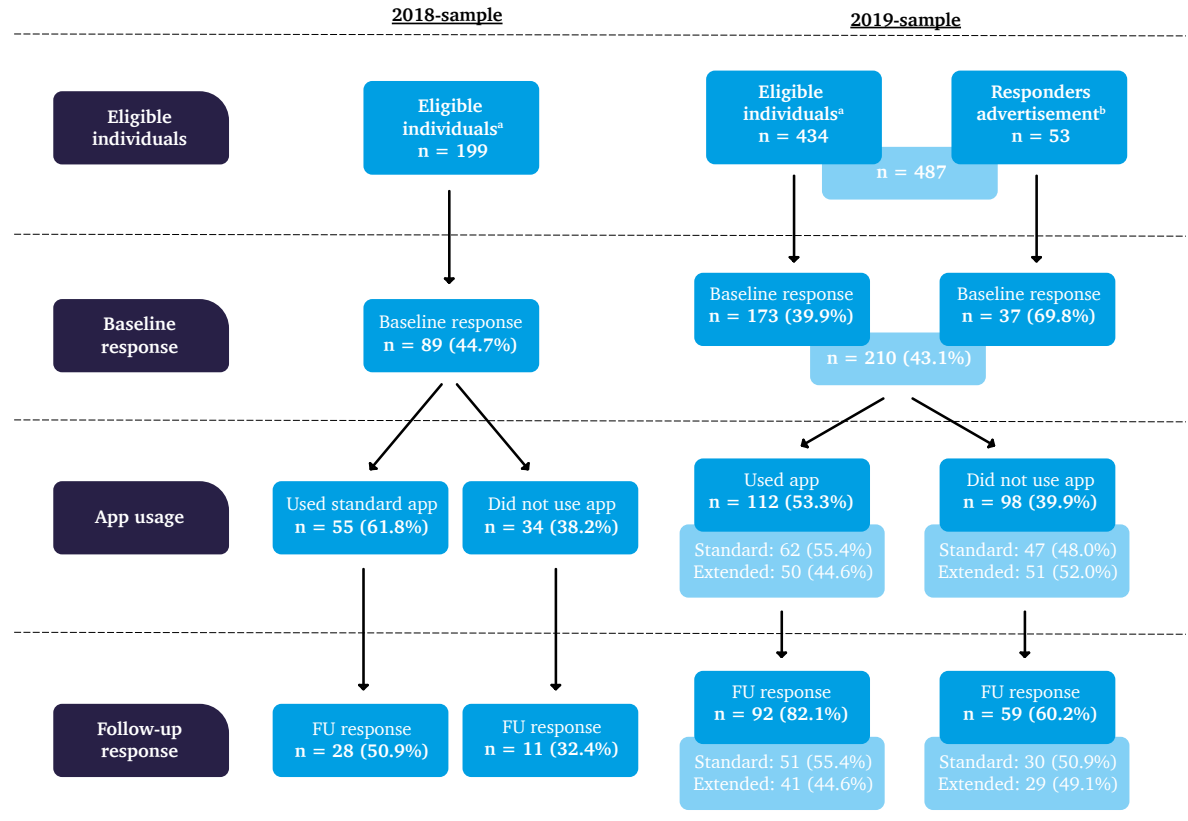

Recruitment via: ${ }^{a}$ eligible individuals from responder samples of surveys evaluating a health campaign and ${ }^{\mathrm{b}}$ advertisement on website Dutch Brain Foundation and newsletter large health care institution in the south of the Netherlands (higher response rate than a because of advertisement format: all reached out to participate themselves). 
Additional File 2. Overview of the questions, instruments and cut-offs for the 12 LIBRA factors

\begin{tabular}{|c|c|c|}
\hline & Questions/instruments & Cut-off \\
\hline $\begin{array}{l}\text { Low-to-moderate } \\
\text { alcohol consumption }\end{array}$ & $\begin{array}{l}\text { What is your daily, average } \\
\text { alcohol consumption? }\end{array}$ & $\begin{array}{l}\text { I don't drink alcohol }(-1.0) \\
\text { I drink up to } 1 \text { glass a day }(-1.0) \\
\text { I drink more than } 1 \text { glass a day } \\
\text { (0) }\end{array}$ \\
\hline $\begin{array}{l}\text { Coronary heart } \\
\text { disease }\end{array}$ & $\begin{array}{l}\text { Has your doctor ever told you } \\
\text { that you have a heart- or blood } \\
\text { vessel condition (including } \\
\text { heart attack, angina (chest } \\
\text { pain), heart failure, stroke or } \\
\text { TIA's)? }\end{array}$ & $\begin{array}{l}\text { Yes }(+1.0) \\
\text { No }(0) \\
\text { I don't know }(0)\end{array}$ \\
\hline Physical inactivity & $\begin{array}{l}\text { European Prospective } \\
\text { Investigation into Cancer and } \\
\text { Nutrition (EPIC) Physical } \\
\text { Activity Questionnaire }\end{array}$ & $\begin{array}{l}\text { Inactive or moderately inactive } \\
(+1.1) \\
\text { Moderately active or active }(0)\end{array}$ \\
\hline $\begin{array}{l}\text { Chronic kidney } \\
\text { disease }\end{array}$ & $\begin{array}{l}\text { Has your doctor ever told you } \\
\text { that you have chronic kidney } \\
\text { disease? }\end{array}$ & $\begin{array}{l}\text { Yes }(+1.1) \\
\text { No }(0) \\
\text { I don't know }(0)\end{array}$ \\
\hline Diabetes & $\begin{array}{l}\text { Has your doctor ever told you } \\
\text { that you have diabetes? }\end{array}$ & $\begin{array}{l}\text { Yes }(+1.3) \\
\text { No }(0) \\
\text { I don't know }(0)\end{array}$ \\
\hline Cholesterol & $\begin{array}{l}\text { Has your doctor ever told you } \\
\text { that your cholesterol is too } \\
\text { high? }\end{array}$ & $\begin{array}{l}\text { Yes }(+1.4) \\
\text { No }(0) \\
\text { I don't know }(0)\end{array}$ \\
\hline
\end{tabular}




\begin{tabular}{|c|c|c|}
\hline \multirow[t]{2}{*}{ Smoking } & Do you smoke? & Yes $(+1.5)$ \\
\hline & & No (0) \\
\hline \multirow[t]{7}{*}{ Obesity } & How long are you (in & $\mathrm{BMI} \geq 30(+1.6)$ \\
\hline & centimetres)? & $\mathrm{BMI}<30(0)$ \\
\hline & How much do you weigh? & \\
\hline & (Round off on whole kilograms. & \\
\hline & Compute body mass index & \\
\hline & $(B M I)=$ weight $(\mathrm{kg}) /$ length & \\
\hline & $\left(m^{2}\right)$ & \\
\hline \multirow[t]{3}{*}{ Hypertension } & Has your doctor ever told & Yes $(+1.6)$ \\
\hline & you that you have high blood & No $(0)$ \\
\hline & pressure? & I don't know (0) \\
\hline Healthy diet/ & Mediterranean Diet Adherence & MEDAS total score $\leq 8(-1.7)$ \\
\hline Mediterranean diet & Screener (MEDAS) & MEDAS total score $<8(0)$ \\
\hline \multirow[t]{2}{*}{ Depression } & Patient Health Questionnaire & PHQ-9 score $>9(+2.1)$ \\
\hline & (PHQ-9) & PHQ-9 score $\leq 9(0)$ \\
\hline \multirow[t]{2}{*}{ High cognitive activity } & Cognitive Reserve Index & CRIq total score $\geq 130(-3.2)$ \\
\hline & questionnaire (CRIq) & CRIq total score $<130(0)$ \\
\hline
\end{tabular}

LIBRA: LIfestyle for BRAin health index. 


\section{Additional File 3. User interface MijnBreincoach app}

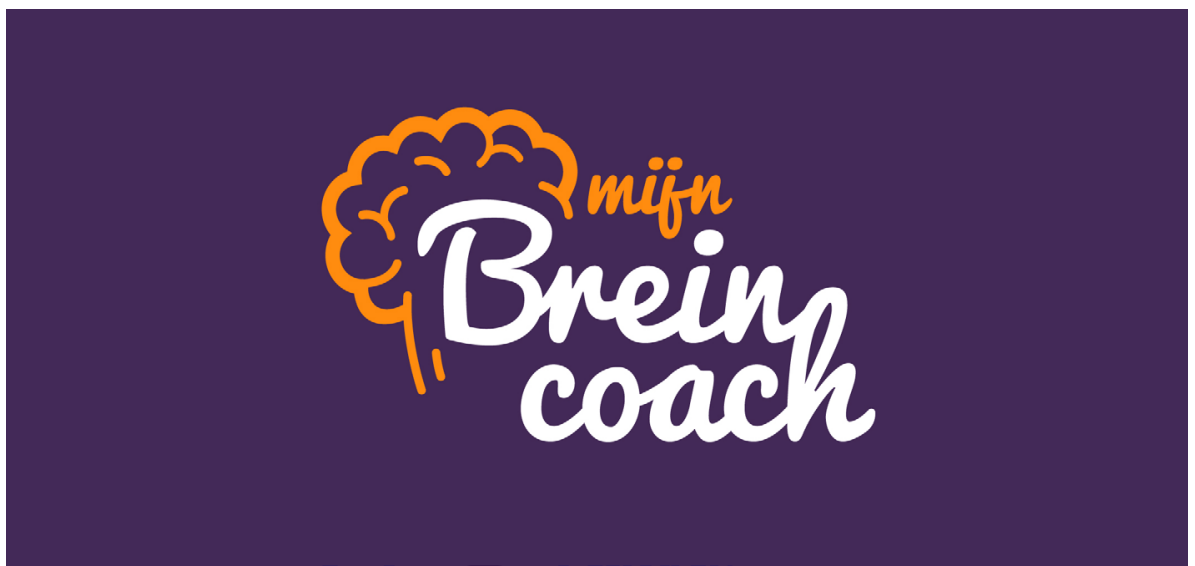

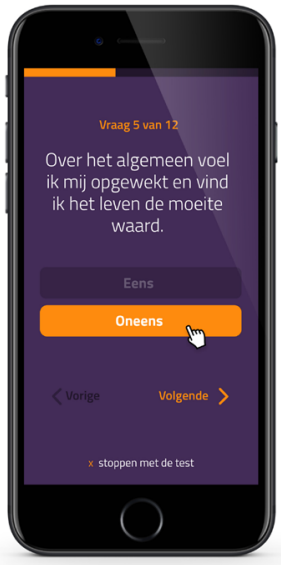

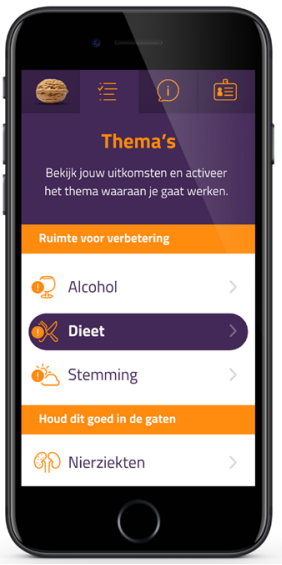

B

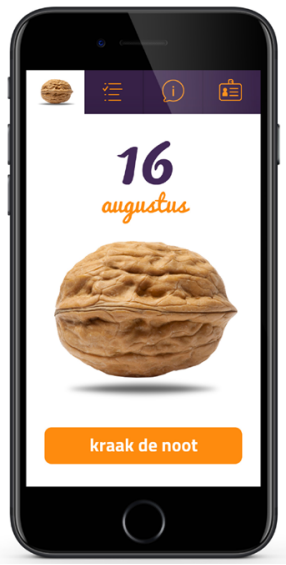

C

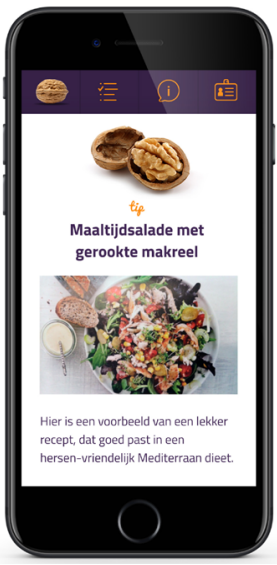

${ }^{\text {a }}$ Completing the LIBRA test; ${ }^{\mathrm{b}}$ Personal brain health profile; ${ }^{\mathrm{c}}$ Presentation of daily notification ("Walnut of the day"); ${ }^{\mathrm{d}}$ Content of daily notification (screenshot from LIBRA theme diet: advice in the form of a brain-healthy recipe). LIBRA: LIfestyle for BRAin health index. 
An mHealth tool for dementia risk reduction: a pre-post proof-of-concept study 

Chapter 7

General discussion

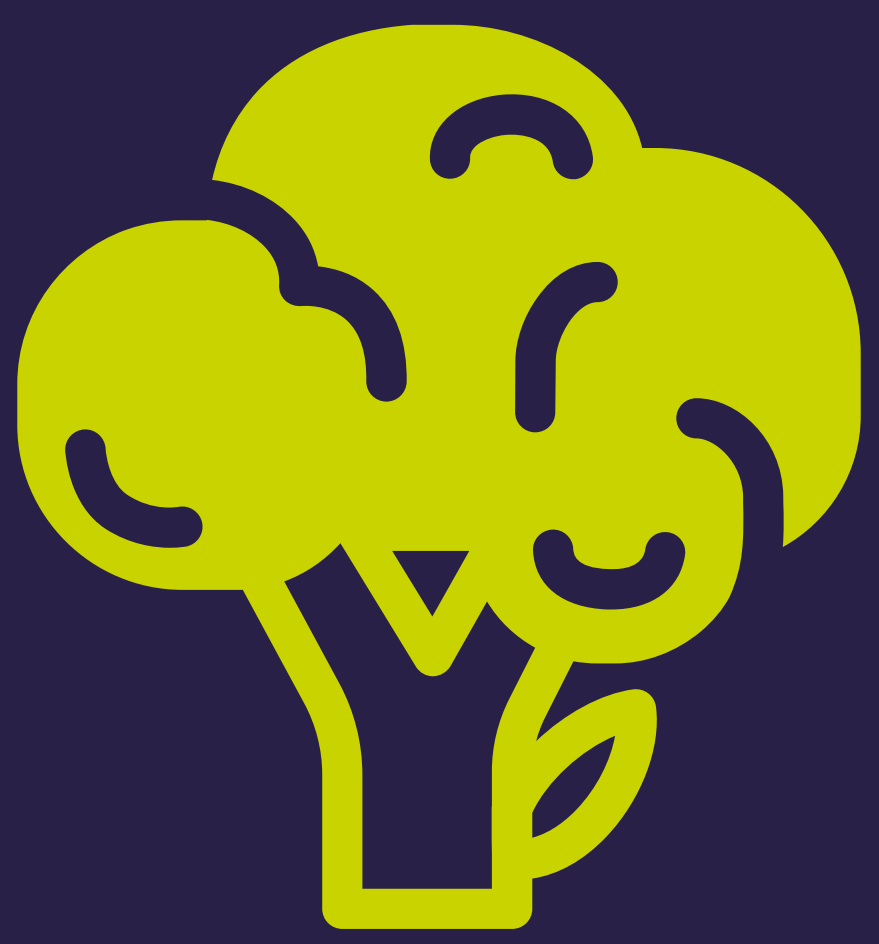





\section{Introduction}

There is a growing body of evidence for risk reduction of dementia via lifestyle modification and cardiovascular risk management, showing the potential of primary prevention of dementia ${ }^{1-4}$. The LIfestyle for BRAin health (LIBRA) index ${ }^{5}$ aims to quantify the modifiable dementia risk to identify at-risk groups and individual room-for-improvement in lifestyle. It is noteworthy that awareness of dementia risk reduction in the general population and knowledge about risk factors is low, and that dementia is often considered by people as being part of normal aging, genetically determined and, thus, non-preventable ${ }^{6,7}$. To use the full potential of dementia risk reduction, it is important to raise awareness on dementia risk reduction in the general population as a crucial first step towards behavioural change.

This final chapter provides a brief overview of the outline of this thesis, describes the main findings, the methodological considerations, implications and future directions.

Part 1 of this thesis focussed on LIBRA. The already well-established association between LIBRA and $\operatorname{cognition}^{8-13}$ was explored in more depth, by looking into underlying biological pathways (i.e., volumetric markers on brain Magnetic Resonance Imaging (MRI)) in Chapter 2 and investigating whether socioeconomic differences influence the relationship between LIBRA and rate of cognitive decline in Chapter 3. Part 2 of this thesis described the development and evaluation of two proof-of-concept studies aimed at raising awareness of dementia risk reduction. Chapter $\mathbf{4}$ and $\mathbf{5}$ described the rationale, development and evaluation of a public health campaign and Chapter 6 evaluated the MijnBreincoach ("MyBraincoach") mobile app.

\section{Main findings}

\section{Part 1: Epidemiological perspective}

In this thesis, we were able to further validate LIBRA as a predictor of cognitive functioning, cognitive decline and cognitive impairment, and to provide first evidence for associated structural brain changes.

In a cross-sectional analysis in The Maastricht Study of 4,164 individuals aged 40 - 75 years old, described in Chapter 2, we found that a higher LIBRA score, reflecting a less brain-healthy lifestyle, was associated with higher volumes of 
white matter hyperintensities, lower scores on information processing speed and executive function and attention and higher odds of cognitive impairment. In men, higher LIBRA scores were also associated with lower volumes of grey matter, higher volumes of cerebrospinal fluid (as a proxy for general brain atrophy) and lower memory function. White matter hyperintensities and cerebrospinal fluid volume partly explained the association between LIBRA and cognitive functioning. This study not only confirmed previous work showing that a higher LIBRA score is related to lower cognitive performance ${ }^{8-13}$, but also provided first evidence at the level of volumetric brain changes. Thus, even when only modifiable factors were considered (and adjusting for age, sex and education), we still find associations with cognitive functioning and brain markers in relatively healthy participants, showing that LIBRA indeed reflects brain health in midlife. Sex differences in the lifestyle-related pathology and manifestations of dementia need to be further explored.

The longitudinal analyses of 1,223 participants aged 40 years or older in the Maastricht Aging Study (MAAS), described in Chapter 3, showed that higher LIBRA scores predicted faster rate of decline in information processing speed and verbal memory function. In addition, participants with lower socioeconomic status (SES; a compound score of educational level and equivalent income) displayed more decline in information processing speed and verbal memory function than participants with higher SES. However, no consistent effect modification between SES and LIBRA was observed over time. This suggests that lifestyle is an important determinant of cognitive decline across all SES strata. Yet, people with low SES had an unfavourable LIBRA score, suggesting that they have more potential to benefit from lifestyle-based interventions.

These two studies underline the importance of intervening on risk factors that directly impact the cardiovascular and metabolic system, and thereby the risk of dementia. Also, these studies show that LIBRA could be useful to capture the modifiable risk fraction and may serve as a participant selection tool and surrogate outcome measure in lifestyle intervention trials. LIBRA can also be used to inform people about target behaviours in primary care and public health initiatives. It is paramount to address socioeconomic differences in developing and executing public health initiatives to reduce the worldwide health inequalities. This can be done on a global level (e.g., by the World Health Organization (WHO) ) and on a national or local level (e.g., by local and national governments and the municipal health services). 


\section{Part 2: Public health perspective}

The focus of this part of the thesis was the development of two proof-of-concept studies aimed at raising awareness of dementia risk reduction. Chapter 4 described the assessment of the level of awareness in a random sample of community-dwelling individuals in the Province of Limburg, the Netherlands, aged 40 to 75 years old. This study served as the rationale for the proof-ofconcept studies described in Chapter 5 and 6. Of all 590 participants, the majority (56\%) stated that dementia risk reduction is not possible, in particular showing gaps of knowledge for the cardiovascular risk factors of dementia. The largest self-reported barrier to engage in a brain-healthy lifestyle was lack of knowledge, and most people expressed a need for brain-health education. Variation in level of awareness by educational level and age was observed. Results of this study stress the need to inform the public about the potential of dementia risk reduction.

Building upon these results, Chapter 5 described the public health campaign that was developed and executed in the Province of Limburg, targeting middle-aged community-dwelling individuals. The main message of this campaign was that a healthy lifestyle benefits brain health. No pre-post difference was observed in population-level awareness of dementia risk reduction. However, two of the three themes that formed the foundation of this campaign ("eat healthy" and "exercise regularly") were identified more often after the campaign as being protective against dementia. From all post-campaign participants of the survey, the group that reported to have been exposed to the campaign was more aware of dementia risk reduction than the non-exposed group. Participants from the community-oriented campaign, which was customized to the needs and wishes of three specific districts, recognized campaign materials and the MijnBreincoach platform more often than participants from the broad, mass media-oriented campaign. While awareness of dementia risk reduction was higher in higher-educated participants compared to lower-educated participants both prior to and after the campaign, awareness only improved in the lower educated group. Recommendations include the involvement of the community in the design, implementation and evaluation (participatory action research), the incorporation into a broader dementia or general health project (e.g., in primary care settings), targeting and tailoring the health messages to specific subgroups (e.g., based on educational level, age, risk status), sensitizing younger people, 
and providing more support on the required behavioural change. Overall, future campaigns should scale up to maximize exposure and engagement in the population.

In Chapter 6, two versions of the MijnBreincoach mobile app were evaluated on usage pattern, user appreciation, perceived knowledge increase and beliefs and attitudes related to dementia risk behaviours. Measurements included selfreported questionnaires and usage-tracking data. The total sample consisted of 299 participants; 198 participants used the standard app that was designed to give people insight into their personal dementia risk profile and identify roomfor-improvement based on LIBRA, and 101 participants used the extended app. The latter was additionally equipped to guide participants further into the process of behaviour change, by addressing beliefs and attitudes about dementia risk reduction. Overall, the app was rated positively, and most participants stated to have improved their knowledge on the potential of dementia risk reduction. Women, younger ( $<60$ years) and low-to-middle educated participants evaluated the app most positive. Importantly, the app was designed to make it suitable for less educated or less health literate people (e.g., prompting people with short and simple messages that appeared automatically). However, very few participants used the additional feature of the extended version of the app. On average, no or only modest pre-post changes were observed on beliefs and attitudes related to dementia risk reduction. Usage-tracking showed that $44 \%$ of our sample did not install the app and technical issues were the most frequent self-reported reason for non-use or drop-out. The usability of the tool should be improved prior to executing further effect studies, since it is impossible to draw definite conclusions about the effect of the tool if participants were not able to use it the way it was initially conceived. Future studies should explore the effect of extended behavioural change aspects in the app.

\section{Methodological considerations}

\section{Strengths}

The work in this thesis combined fundamental, epidemiological research, aimed at improving our understanding of modifiable risk and protective factors of dementia (Chapter 2 and 3), with applied research, directed at developing and evaluating ways (e.g., public health campaign, mobile app) to translate this knowledge into practice and policy (Chapters 4-6). 
The use of relatively large population-based cohort studies (The Maastricht Study and the Maastricht Aging Study) to study LIBRA in Chapter 2 and Chapter 3 increased the power to detect underlying associations. The target age range of 40 years and older makes the results of these studies particularly generalizable to middle-aged individuals, which seems the most feasible age bracket to target most risk and protective factors. The extensive phenotyping approach of risk factors, multi-domain cognitive outcomes, and availability of state-of-the-art population imaging data in The Maastricht Study (Chapter 2) allowed us to study underlying lifestyle-brain pathways in good depth. Hereby, we took the non-linear association between LIBRA and cognition into account in the mediation analysis ${ }^{14}$ by quantifying the change in cognition through MRI markers across different levels of LIBRA. This provided an interesting and new approach to the data compared to traditional mediation analyses in which direct and indirect effects are decomposed. The longitudinal design used in Chapter 3 made it possible to focus on the rate of decline of cognitive functioning over time. The use of a compound score for socioeconomic status, in which not only an educational measure (which is a proxy for cognitive reserve ${ }^{15}$ ) but also an economical measure (equivalent income) was considered, is beneficial for the construction of a valid and robust SES construct.

The proof-of-concept studies on the effects of a public health campaign and the MijnBreincoach mobile app on increasing public awareness of brain health, described in Chapter 4, 5 and 6, were built upon previous research by using comparable methodology. For example, the British Social Attitudes survey for assessment of dementia risk awareness ${ }^{7}$, and the Motivation to Change Lifestyle and Health Behaviours for Dementia Risk Reduction scale ${ }^{16}$ were used. Regarding the design of the public health initiatives (awareness campaign and app), both addressed multiple dementia risk factors (based on LIBRA) and were developed in collaboration with stakeholders in a multidisciplinary and multisectoral approach, fully in line with guidelines of the $\mathrm{WHO}^{17}$. The major partners we consulted in these projects were the municipal health services, the department of Health Promotion of Maastricht University, an agency for strategy and design (Zuiderlicht), two software companies (Betawerk and Sananet), the Dutch Alzheimer's Association and the Dutch Brain Foundation. The campaign materials and custom-made daily notifications of the app were checked on accuracy, completeness, and readability by renowned experts in the field, and on general acceptance in potential end-users and a client panel of the Alzheimer Centre Limburg. 


\section{Limitations}

This thesis had several limitations that need to be addressed. In both LIBRA validation studies, LIBRA was calculated based on 11 instead of all 12 factors. The absence of the LIBRA factor cognitive activity, which is the strongest protective factor with a weight of -3.2, in The Maastricht Study (Chapter 2) and of the LIBRA factor healthy diet (weight of -1.7) in the Maastricht Aging Study (Chapter 3) could have weakened the predictive value of LIBRA ${ }^{5}$. A general note regarding the LIBRA calculation is that the use of dichotomous scores, i.e., presence of LIBRA factor yes/no, makes the index less suitable to detect small changes in a specific factor in behavioural change programs. Yet, a study showed that LIBRA was most responsive to change compared to other risk indices, probably due to the large amount of modifiable factors and omission of non-modifiable factors ${ }^{18}$. Further, the mediation analyses described in Chapter $\mathbf{2}$ are of cross-sectional nature and should be replicated in a prospective design. In addition, the non-linear mediation analyses, which calculated instantaneous mediation effects at specific values of LIBRA, did not allow for traditional decomposition of direct and indirect effects. This made it impossible to quantify the overall indirect effect of LIBRA on cognition through the MRI markers.

The design of the studies and used measurements induced bias that needs to be addressed. Measurements in the two cohort studies (Chapter 2 and 3) were operationalized as much as possible by objective and/or clinical data from assessment at the research clinic or patient registries. However, only selfreported data were available for some LIBRA factors (e.g., physical activity) and socioeconomic status (i.e., self-reported net month income), which could have led to recall bias or socially desirable responses. Furthermore, selection bias may have occurred in the studies, which is an unfortunate but common phenomenon in ageing studies. In Chapter 2, we excluded participants who did not undergo an MRI scan. This group was older and more frail, which might have led to an underestimation of the true association in the population. In Chapters 4, 5 and 6, we recruited participants for the proof-of-concept studies from a responder sample of people with a specific interest in health research. This has probably affected the strength of the associations, for example by overestimating awareness of dementia risk reduction and the acceptance and potential of the use of Internet applications in the general population. Furthermore, the used questionnaire to assess presence of "engagement in a cognitively active lifestyle" in the MijnBreincoach app (Chapter 6), the 
Cognitive Reserve Index questionnaire (CRIq) ${ }^{19}$, allows older people to have more chance of higher (i.e., better) scores because of accumulated activities over the years. This makes this index less suitable to detect current roomfor-improvement to engage in cognitively stimulating activities, independent of your age or your history. This challenge in using the CRIq and alternative questionnaires for assessing cognitive activity will be explored in the near future. Last, it should be noted that the internal validity of the MijnBreincoach study is low since we did not use a control group and randomization (in the total sample).

\section{Implications}

Since the development of LIBRA in 2015 by Maastricht University, much research has been conducted in order to validate LIBRA for cognitive outcomes (i.e., functioning, decline and impairment) and dementia risk ${ }^{8-13}$. Further, LIBRA has proven to be responsive to change and intervention effects in randomized controlled trials ${ }^{13,18}$. Whereas other risk indices selected risk factors based on single cohort studies (i.e., the CAIDE Score ${ }^{20}$ ), which limits the detection of dementia risk factors to the variables included in the dataset, or on an existing literature search (i.e., ANU-ADRI ${ }^{21}$ ), LIBRA is based on a careful systematic development, including a comprehensive literature review and international Delphi consensus rounds. It should be noted that LIBRA has, compared to other risk indices, per definition less overall predictive accuracy since it is does not incorporate age as a risk factor (or any other unmodifiable factors), which is known to be the most potent predictor variable in these indices ${ }^{22}$. The fact that, even when only including modifiable factors and adjusting for age, sex and educational level, we still were able to find associations with cognitive performance, dementia risk and underlying biological gradients of volumetric brain markers, proves that LIBRA is indeed an index for brain health and has great public health implications. Given that around $40 \%$ of all dementia cases worldwide is attributable to factors that could potentially be delayed or prevented underlines the impact of these implications. LIBRA could serve as an important public health tool and, amongst others, be used in primary care settings to identify at-risk individuals who will benefit most from cardiovascular risk management and lifestyle interventions and to monitor lifestyle and health status in prevention trials and health campaigns.

Our awareness campaign was the first public health initiative in the Netherlands aimed at raising awareness of dementia risk reduction. It was designed as a 
proof-of-concept study to identify both the barriers and facilitators of executing a public health campaign. The fact that, even with limited resources, our campaign was able to raise awareness on two out of the three campaign themes is promising. Also, we experienced a large support-base for our campaign from stakeholders within the Province, creating a "snowball effect" in spreading the campaign message. Although we had chosen a flexible and open design for the campaign, in which it was possible to alter strategies along the way, the underlying theoretical framework of the campaign was based on the Theory of Planned Behaviour ${ }^{23}$ 24. According to this theory, beliefs about the likely consequences of the behaviour (behavioural beliefs), about the expectations of others (normative beliefs) and about the presence of facilitators and barriers to engage in the behaviour (control beliefs) influence the intention to perform the behaviour ${ }^{23,24}$. In our campaign, we addressed these beliefs by providing information on the role of a healthy lifestyle on lowering dementia risk (e.g., campaign website, public events, personal risk profiler in the MijnBreincoach mobile app) and providing simple and positive examples of how to engage in such behaviours (e.g., campaign posters and daily notifications in the MijnBreincoach app). We are aware that these are important yet insufficient steps towards the behavioural change that is needed to reach the potential of dementia risk reduction. However, our primary goal was to assess the level of awareness, to explore the needs, wishes and barriers to learn more about and to engage in a brain-healthy lifestyle and to subsequently raise awareness on this matter in community-dwelling inhabitants of the Province of Limburg, the Netherlands. Our broader aim was to also raise awareness among regional and national policymakers of the fact that the time is ripe to act on dementia risk reduction and to facilitate the joining of forces on these initiatives.

\section{Future directions}

\section{LIBRA and MijnBreincoach}

There are many opportunities for LIBRA and MijnBreincoach in future research, and several projects have already been planned or launched based on the findings presented in this thesis. The Dutch multi-domain lifestyle intervention FINGER-NL, in which participants are either randomized to a high-intensity or low-intensity group for a duration of 24 months, was launched in 2021. It uses LIBRA as a secondary outcome measure. Furthermore, the MijnBreincoach app, that has so far been tested in the general population, will be tested in primary care settings in a new and ongoing study, and is currently translated 
into German, French and Danish for use in national projects. The public health campaign tested in Limburg in currently running in other regions in the Netherlands. Next, since LIBRA is based on a systematic literature review dating back from 2015, it is important to keep the index up to date using recently obtained new insights. Therefore, the mixed methods approach conducted in 2015 will be repeated, which could lead to the inclusion of new risk and protective factors (e.g., sleep or auditory function). The recently launched ABOARD (A Personalized Medicine Approach for Alzheimer's Disease) project, by a consortium of more than thirty Dutch partners aimed at stopping dementia before it starts, will adapt the existing LIBRA for use in the clinical population by triangulation of the available evidence. An online risk profiler will be developed showing patients' most relevant risk factors and inform them about their room-for-improvement, based on the MijnBreincoach mobile app for the general population.

Next, it could also be of value to investigate ways to make LIBRA more suitable to detect small behavioural changes. A recent study that investigated the responsiveness of LIBRA in three large multi-domain dementia prevention trials, proposed (weighted) Z-score versions of the modifiable risk factors in LIBRA and other risk indices instead of the traditional dichotomous scores (i.e., presence of LIBRA factors yes/no) to make them more suitable as surrogate outcomes measures in lifestyle intervention trials ${ }^{18}$. Methods using data science and machine learning could also be of interest in this respect ${ }^{18}$. Furthermore, given the interactions between the different LIBRA factors (e.g., hypertension and coronary heart disease, obesity and physical inactivity) and the fact that our LIBRA validation studies so far have not taken these interactions into account, it would be interesting to model possible interactions between LIBRA factors in our algorithm and see whether the found associations with cognitive and structural brain outcomes remain.

\section{Raising awareness}

It would be interesting to assess the level of awareness of dementia risk reduction and the factors associated with motivation to change behaviour for dementia risk reduction in a clinical sample (i.e., visitors of memory clinics). The use of (adapted versions of) our surveys (Chapter $4,5,6$ ) could provide insights into dementia risk literacy, preferences for seeking information on this topic, barriers to engage in a healthy lifestyle and predictors of successful behavioural change in a population that might be at-risk for developing dementia. This 
would mean a shift from primary prevention of dementia towards secondary prevention. An important consideration in conducting such projects would be to avoid "victim blaming" and stigmatization ${ }^{25}$, by emphasizing on the positive message of room-for-improvement and to take ethical considerations into account. Collaboration with important stakeholders (e.g., Dutch Alzheimer's Association), and co-creation with and pilots in the target group are therefore important.

\section{Conclusions}

This thesis provided important insights into the further validation of LIBRA, consisting solely of modifiable factors, for cognitive decline and impairment and volumetric brain changes. Next, this thesis described the first public health campaign in the Netherlands aimed at raising awareness of dementia risk reduction and described an mHealth intervention (MijnBreincoach) that identifies individual room-for-improvement and informs about the relationship between lifestyle and brain health. We hope that this thesis will contribute to the joining of forces to disseminate the existing evidence on dementia risk reduction to the general public. 


\section{References}

1. Norton S, Matthews FE, Barnes DE, Yaffe K, Brayne C. Potential for primary prevention of Alzheimer's disease: an analysis of population-based data. Lancet Neurol 2014;13:788-94.

2. Lincoln $\mathrm{P}$, Fenton $\mathrm{K}$, Alessi $\mathrm{C}$, et al. The Blackfriars Consensus on brain health and dementia. Lancet 2014;383:1805-06.

3. Baumgart M, Snyder HM, Carrillo MC, Fazio S, Kim H, Johns H. Summary of the evidence on modifiable risk factors for cognitive decline and dementia: A population-based perspective. Alzheimers Dement 2015;11:718-26.

4. Livingston G, Huntley J, Sommerlad A, et al. Dementia prevention, intervention, and care: 2020 report of the Lancet Commission. Lancet 2020;396:413-46.

5. Deckers K, van Boxtel MP, Schiepers OJ, et al. Target risk factors for dementia prevention: a systematic review and Delphi consensus study on the evidence from observational studies. Int $J$ Geriatr Psychiatry 2015;30:234-46.

6. Cations M, Radisic G, Crotty M, Laver KE. What does the general public understand about prevention and treatment of dementia? A systematic review of population-based surveys. PLoS One 2018;13.

7. Marcinkiewicz A, Reid S, Attitudes to dementia: Findings from the 2016 British Social Attitudes survey 2016, NatCen Social Research: London.

8. Vos SJB, van Boxtel MPJ, Schiepers OJG, et al. Modifiable Risk Factors for
Prevention of Dementia in Midlife, Late Life and the Oldest-Old: Validation of the LIBRA Index. $J$ Alzheimers Dis 2017;58:537-47.

9. Schiepers OJG, Kohler S, Deckers K, et al. Lifestyle for Brain Health (LIBRA): a new model for dementia prevention. Int $J$ Geriatr Psychiatry 2018;33:167-75.

10. Pons A, LaMonica HM, Mowszowski L, Kohler S, Deckers K, Naismith SL. Utility of the LIBRA Index in Relation to Cognitive Functioning in a Clinical Health Seeking Sample. J Alzheimers Dis 2018;62:373-84.

11. Deckers $K$, Nooyens $A$, van Boxtel M, Verhey F, Verschuren M, Kohler S. Gender and Educational Differences in the Association between Lifestyle and Cognitive Decline over 10 Years: The Doetinchem Cohort Study. J Alzheimers Dis 2018;70:S31-S41.

12. Deckers $\mathrm{K}$, Cadar $\mathrm{D}$, van Boxtel MPJ, Verhey FRJ, Steptoe A, Kohler S. Modifiable Risk Factors Explain Socioeconomic Inequalities in Dementia Risk: Evidence from a Population-Based Prospective Cohort Study. J Alzheimers Dis 2019;71:549-57.

13. Deckers $\mathrm{K}$, Köhler $\mathrm{S}$, Ngandu $\mathrm{T}$, et al. Quantifying dementia prevention potential in the FINGER randomized controlled trial using the LIBRA prevention index. Alzheimers Dement 2021.

14. Hayes AF, Preacher KJ. Quantifying and Testing Indirect Effects in Simple 
Mediation Models When the Constituent Paths Are Nonlinear. Multivariate Behav Res 2010;45:627-60.

15. George KM, Lutsey PL, Kucharska-Newton A, et al. Life-Course Individual and Neighborhood Socioeconomic Status and Risk of Dementia in the Atherosclerosis Risk in Communities Neurocognitive Study. Am J Epidemiol 2020;189:113442.

16. Kim S, Sargent-Cox K, Cherbuin N, Anstey KJ. Development of the motivation to change lifestyle and health behaviours for dementia risk reduction scale. Dement Geriatr Cogn Dis Extra 2014;4:172-83.

17. Risk reduction of cognitive decline and dementia: WHO guidelines. 2019, World Health Organization: Geneva.

18. Coley N, Hoevenaar-Blom MP, van Dalen JW, et al. Dementia risk scores as surrogate outcomes for lifestyle-based multidomain prevention trials-rationale, preliminary evidence and challenges. Alzheimers Dement 2020;16:1674-85.

19. Nucci M, Mapelli D, Mondini S. Cognitive Reserve Index questionnaire (CRIq): a new instrument for measuring cognitive reserve. Aging Clin Exp Res 2012;24:21826.

20. Kivipelto $M$, Ngandu T, Laatikainen T, Winblad B, Soininen $\mathrm{H}$, Tuomilehto J. Risk score for the prediction of dementia risk in 20 years among middle aged people: a longitudinal, population-based study. Lancet Neurol 2006;5:735-41.

21. Anstey KJ, Cherbuin N, Herath PM. Development of a new method for assessing global risk of Alzheimer's disease for use in population health approaches to prevention. Prev Sci 2013;14:411-21.

22. Licher S, Yilmaz P, Leening MJG, et al. External validation of four dementia prediction models for use in the general community-dwelling population: a comparative analysis from the Rotterdam Study. Eur J Epidemiol 2018;33:645-55.

23. Ajzen I. Theory of Planned Behavior. https://people.umass.edu/aizen/index. html (May 11, 2021, date last accessed).

24. Godin G, Kok G. The theory of planned behavior: a review of its applications to health-related behaviors. AJHP 1996;11:87-98.

25. Horstkötter D, Deckers K, Köhler S. Primary Prevention of Dementia: An Ethical Review. $J$ Alzheimers Dis 2021;79:467-76. 
General discussion 

Addendum

Summary

Nederlandse samenvatting

Impact paragraph

List of publications

Dankwoord

Thesis defences of MHeNs

Author information 

Summary

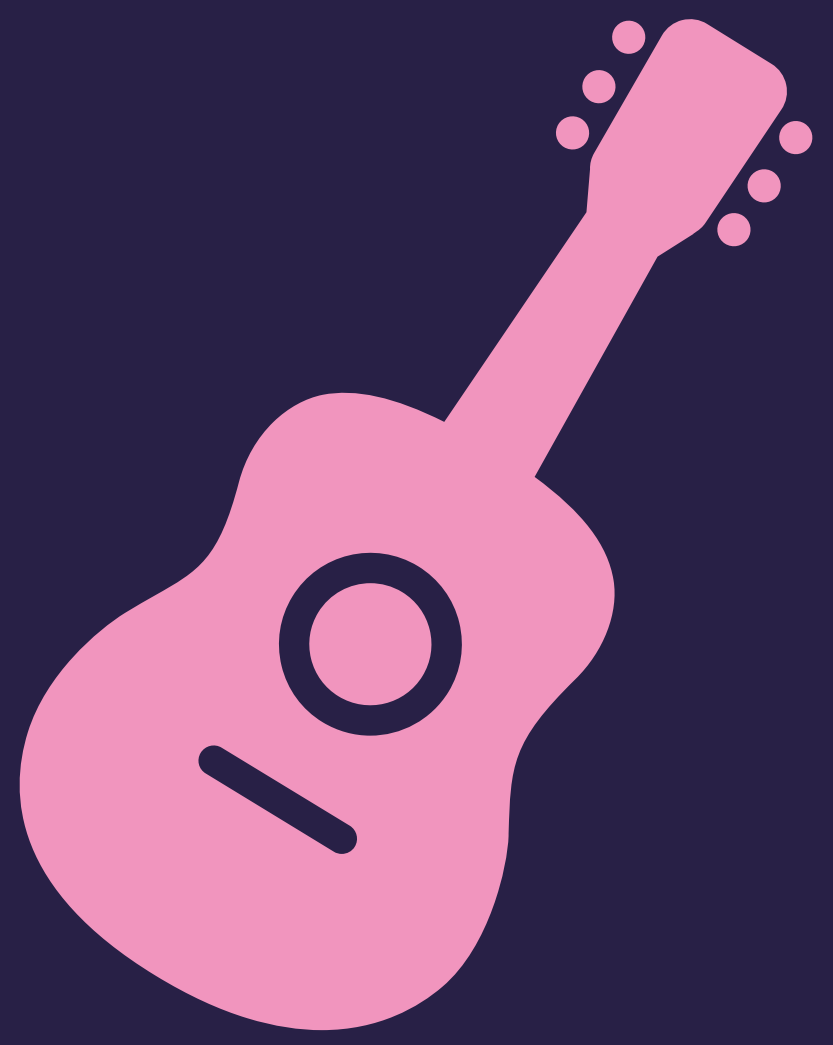





\section{Summary}

A substantial proportion of dementia cases worldwide is attributable to modifiable risk factors such as physical inactivity, smoking and high blood pressure, stressing the potential of primary prevention of dementia. Maastricht University developed the LIfestyle for BRAin health (LIBRA) index in 2015, a risk score consisting of twelve modifiable risk and protective factors for cognitive decline and dementia. LIBRA has been shown to predict cognitive decline and dementia risk in several cohort studies and could be used as a tool to inform people about their personal dementia (modifiable) risk profile and room-for-improvement, and to identify at-risk groups in health campaigns. However, the underlying biologically plausible mechanisms for the association of LIBRA with cognition, such as neurodegeneration and cerebrovascular damage, have not been explored to date. Hence, the main aim of the first part of this thesis was to contribute to the existing evidence of the association between LIBRA and cognition, by exploring potential plausible underlying pathways of this association using volumetric brain markers. In addition, we aimed to investigate whether the association between LIBRA and cognitive decline differs across different levels of socioeconomic status (SES).

It is remarkable that the scientific evidence for a healthy lifestyle in delaying or even preventing dementia onset has barely been disseminated to the general population. Most people in the general public are still unaware of the potential of dementia risk reduction and view dementia as non-preventable and part of normal aging. These gaps in knowledge can pose barriers to engage in behaviour change to reduce dementia risk, thereby not using the full potential of dementia risk reduction. The second part of this thesis aimed to assess awareness of dementia risk reduction in the general population and to identify the needs, wishes and barriers to engage in a brain-healthy lifestyle. Subsequently, we aimed to raise awareness in the general population by conducting two proof-ofconcept studies (a public health awareness campaign and the MijnBreincoach app), to identify the facilitators and barriers for future projects. In Chapter 1, a general introduction is given into the topic of dementia risk reduction and aims of this thesis.

\section{Part 1: Epidemiological perspective}

The cross-sectional analyses of The Maastricht Study described in Chapter 2 investigated whether higher (i.e., more unhealthy) LIBRA scores were associated with lower cognitive performance, lower volumes of brain structures 
and more white matter hyperintensities. In addition, we explored whether the MRI damage markers explained the association between LIBRA and cognition. The results showed that higher LIBRA scores were associated with higher volumes of white matter hyperintensities, lower scores on information processing speed and executive function and attention and higher odds of cognitive impairment. In men only, associations between LIBRA and volumes of grey matter and cerebrospinal fluid and memory function were found, too. White matter hyperintensities (a marker of cerebral small vessel disease) and intracranial volume-adjusted cerebrospinal fluid volumes (as a marker for general brain atrophy) partly explained the association between LIBRA and cognitive performance.

Chapter 3 presented the longitudinal analyses of LIBRA in the Maastricht Aging Study, in which we analysed whether the rate of cognitive decline differed across SES strata. A higher (i.e., more unhealthy) LIBRA score and a lower SES (based on a compound score of educational level and net month income) independently predicted more decline in information processing speed and memory function. Across all SES strata, lifestyle appeared to be an important determinant of cognitive decline. Yet, people with low SES had a higher LIBRA score suggesting more potential for lifestyle-based interventions.

\section{Part 2: Public health perspective}

Chapter 4 presented the assessment of literacy of dementia risk reduction in community-dwelling individuals in the province of Limburg, the Netherlands and showed that most individuals were unaware of the relationship between lifestyle and brain health. Specific gaps in knowledge existed regarding the cardiovascular risk factors of dementia, such as high blood pressure and high cholesterol. Awareness was lowest in individuals with a lower educational level and higher age. Lack of knowledge appeared to be the largest barrier to engage in a brain-healthy lifestyle. Most people were eager to learn more about dementia risk reduction and were positive about using a dementia risk reduction eHealth device.

Building upon these results, Chapter 5 described the 10-month public health awareness campaign in the province of Limburg. Two out of the three campaign themes were recognized as being protective against dementia more often after the campaign ("eat healthy" and "exercise regularly") than before the campaign. No population-level change in level of awareness was observed 
after the campaign. Yet, post-campaign participants who had heard of the campaign were more aware of dementia risk reduction than post-campaign participants who had not heard of the campaign. In this respect, it should be noted that this campaign was developed based on limited resources, and largely depended on word-of-mouth and stakeholders philanthropy. In order to reach population-level effects, it is thus important to scale-up and increase resources to reach out. Lessons learned and recommendations for future projects were discussed, such as the incorporation of a prevention campaign into a broader dementia or general health campaign, overcoming the challenge of reaching younger people with a message on dementia (prevention) and the notice that raising awareness is an important, yet insufficient step towards the behavioural change that is needed.

Chapter 6 presented the MijnBreincoach proof-of-concept study, in which participants used the MijnBreincoach app for three months to increase knowledge and motivation to change. Results showed that most users rated the app positively. However, a large part of the sample reported technical issues, which made it difficult to draw conclusions about the effect of the app. Thus, although most users seemed positive about (the concept of) the app, the usability of MijnBreincoach should be improved prior to executing further intervention studies.

The general findings of this thesis, the methodological considerations and the implications and future directions were discussed in Chapter 7. 

Nederlandse samenvatting

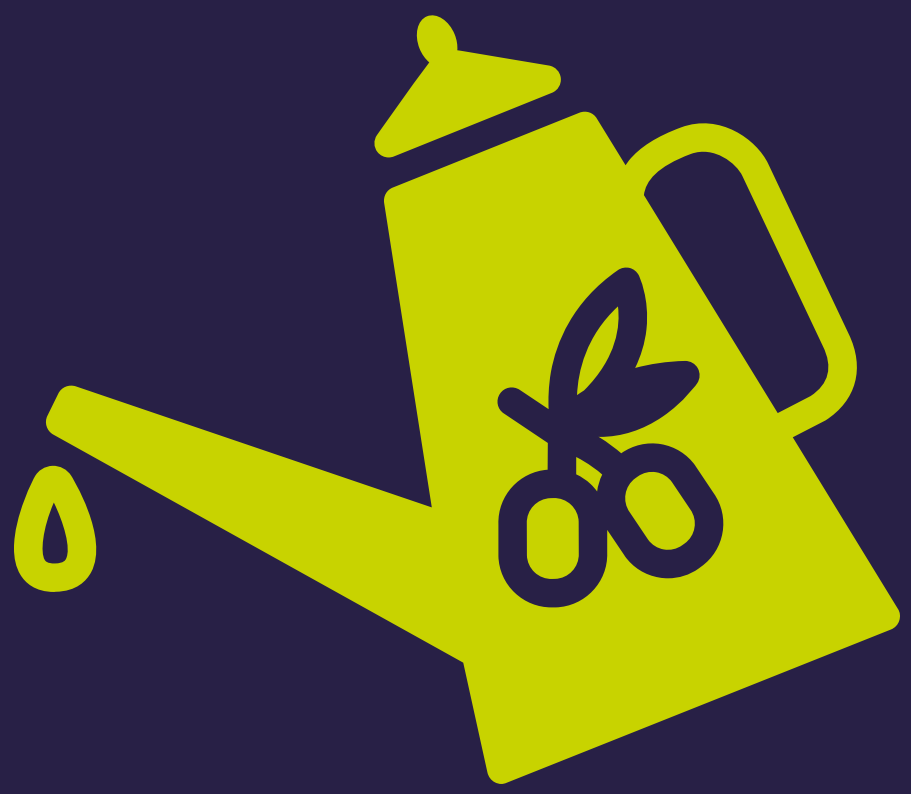





\section{Nederlandse samenvatting}

Een aanzienlijk deel van alle gevallen van dementie wereldwijd is toe te schrijven aan beïnvloedbare risicofactoren, zoals fysieke inactiviteit, roken en hoge bloeddruk, wat het belang van primaire preventie van dementie onderstreept. De Universiteit van Maastricht heeft in 2015 de LIfestyle for BRAin health (LIBRA) index ontwikkeld. Dit is een risico score bestaande uit twaalf mogelijk beïnvloedbare risico- en beschermende factoren voor cognitieve achteruitgang en dementie. Verschillende wetenschappelijke studies laten zien dat LIBRA het risico op cognitieve achteruitgang en dementie voorspelt. LIBRA zou daarmee geschikt kunnen zijn om mensen te informeren over hun persoonlijke (beïnvloedbare) risicoprofiel, inzicht te geven in hun ruimte voor verbetering en om hoog-risicogroepen te identificeren voor gezondheidscampagnes. Er is echter nog nooit onderzocht welke onderliggende biologische mechanismen een rol spelen bij de relatie tussen LIBRA en cognitieve prestaties. Het hoofddoel van het eerste deel van deze thesis was dan ook om bij te dragen aan het bestaande bewijs voor de relatie tussen LIBRA en cognitieve prestaties, door mogelijke onderliggende biologische mechanismes die deze associatie verklaren te exploreren middels MRI-markers. Daarnaast werd onderzocht of de relatie tussen LIBRA en achteruitgang op cognitief functioneren verschilt tussen mensen met een verschillende sociaaleconomische status (SES).

Het is opmerkelijk dat de meeste mensen uit de algemene bevolking nog niet of nauwelijks kennis hebben genomen van het wetenschappelijke bewijs voor de invloed van een gezonde leefstijl op het vertragen dan wel voorkomen van het ontstaan van dementie. Er wordt veel gedacht dat dementie niet-beïnvloedbaar en onderdeel van normale veroudering is. Deze hiaten in kennis kunnen ervoor zorgen dat mensen niet het gedrag uitoefenen dat hun risico zo veel mogelijk verkleint, en daarmee wordt de potentie van risicoreductie van dementie niet volledig benut. Het tweede deel van deze thesis richtte zich daarom op het meten van het niveau van bewustwording over risicoreductie van dementie in de algemene Limburgse bevolking en het in kaart brengen van de wensen, behoeften en barrières om hersengezonder te leven. Vervolgens richtte dit deel van de thesis zich op het verhogen van bewustwording over risicoreductie van dementie in de algemene bevolking door twee proof-of-concept studies uit te voeren (een bewustwordingscampagne en de MijnBreincoach app), om zo de faciliterende en bemoeilijkende factoren te identificeren voor vervolgprojecten. In Hoofdstuk 1 van deze thesis werd een algemene introductie gegeven in het onderwerp risicoreductie van dementie. 


\section{Deel 1: Epidemiologisch perspectief}

In Hoofdstuk 2 werd de cross-sectionele analyse uit De Maastricht Studie beschreven, waarin werd onderzocht of hogere (d.w.z. ongezondere) LIBRA-scores samenhangen met lagere volumes aan breinstructuren, meer witte stof hyperintensiteiten en lagere cognitieve prestaties. Daarnaast werd onderzocht of de MRI-markers de relatie tussen LIBRA en cognitie verklaarden. Uit de resultaten bleek dat hogere LIBRA-scores geassocieerd waren met meer volume aan witte stof hyperintensiteiten, lagere scores op informatieverwerkingssnelheid en executief functioneren en een hogere kans op cognitieve stoornissen. Alleen bij mannelijke deelnemers werd er ook een associatie gevonden tussen LIBRA en volumes van grijze stof en cerebrospinale vloeistof en geheugen. Witte stof hyperintensiteiten (een marker van cerebrale kleine vaatziekten) en intracranieel volume-gecorrigeerd cerebrospinale vloeistof volume (als marker voor algehele breinatrofie) verklaarden de relatie tussen LIBRA en cognitie deels.

In Hoofdstuk 3 werd onderzocht of de relatie tussen LIBRA en cognitieve prestaties beïnvloed werd door SES, middels een longitudinale analyse in de Maastricht Ageing Study. Hogere (d.w.z., ongezondere) LIBRA-scores en lagere SES (op basis van opleidingsniveau en netto maandinkomen) bleken beiden voorspellers van achteruitgang in informatieverwerkingssnelheid en geheugen. Binnen alle SES-groepen bleek leefstijl een belangrijke determinant in het voorspellen van cognitieve achteruitgang. Deelnemers met een lage SES hadden hogere LIBRA-scores, wat suggereert dat zij meer ruimte voor verbetering hebben bij leefstijlinterventies.

\section{Deel 2: Public health perspectief}

In Hoofdstuk 4 werd de steekproefmeting beschreven naar kennis over risicoreductie van dementie bij inwoners uit de provincie Limburg. Hieruit bleek dat het merendeel van deze groep zich niet bewust was van de relatie tussen leefstijl en hersengezondheid en dat er met name kennishiaten waren bij de cardiovasculaire risicofactoren van dementie, zoals hoge bloeddruk en verhoogd cholesterolgehalte. Bewustwording was het laagst bij mensen met een laag opleidingsniveau en hogere leeftijd. Het onderzoek liet verder zien dat de grootste barrière om met een hersengezonde leefstijl aan de slag te gaan een gebrek aan kennis hierover was. De meeste deelnemers gaven aan graag meer te willen leren over dementie risicoreductie en waren ook positief tegenover het gebruik van eHealth. 
Voortbouwende op deze resultaten, evalueerde Hoofdstuk $\mathbf{5}$ een tien maanden durende bewustwordingscampagne in de provincie Limburg. Twee van de drie campagne speerpunten werden na afloop van de campagne vaker herkend als beschermend tegen dementie dan ervoor ("eet gezond" en "beweeg regelmatig"). Op populatieniveau werd geen pre-post verschil geobserveerd in mate van bewustwording van het verminderen van het risico op dementie. In een aanvullende analyse bleek echter wel dat de groep mensen die na afloop van de campagne aangaven met de campagne in aanraking te zijn gekomen, zich meer bewust waren van het verminderen van het risico op dementie dan de groep mensen die niet in aanraking waren gekomen met de campagne. Een belangrijke kanttekening is dan ook dat deze campagne tot stand is gekomen met beperkte middelen en grotendeels afhankelijk was van mond-tot-mond reclame en de inzet van belanghebbenden. Om veranderingen op populatieniveau te bewerkstelligen is maximalisering van het bereik van campagnes noodzakelijk. Aanbevelingen voor vervolgprojecten zijn in het artikel besproken, zoals het toevoegen van een boodschap over preventie aan een bredere dementie- of gezondheidscampagne, het aangaan van de uitdaging om jongere groepen te bereiken met een boodschap over dementie (preventie) en de kanttekening dat bewustwording verhogen een belangrijke maar onvolledige stap is richting de benodigde gedragsverandering.

Hoofdstuk 6 beschrijft de MijnBreincoach proof-of-concept studie, waarin deelnemers drie maanden gebruik maakten van de MijnBreincoach app om kennis over en motivatie voor risicoreductie van dementie te verhogen. De meeste gebruikers evalueerden de app positief. Echter, een groot deel van de deelnemers rapporteerden technische problemen binnen de app, waardoor het lastig was om conclusies te trekken over het effect. Alhoewel de meeste gebruikers positief waren over (het concept van) de app, dient de gebruiksvriendelijkheid verbeterd te worden voordat nieuwe interventiestudies naar MijnBreincoach plaats kunnen vinden.

In Hoofdstuk 7 werden de algemene bevindingen uit deze thesis, de gebruikte methoden en de implicaties en toekomstvisie besproken. 



\section{Impact paragraph}

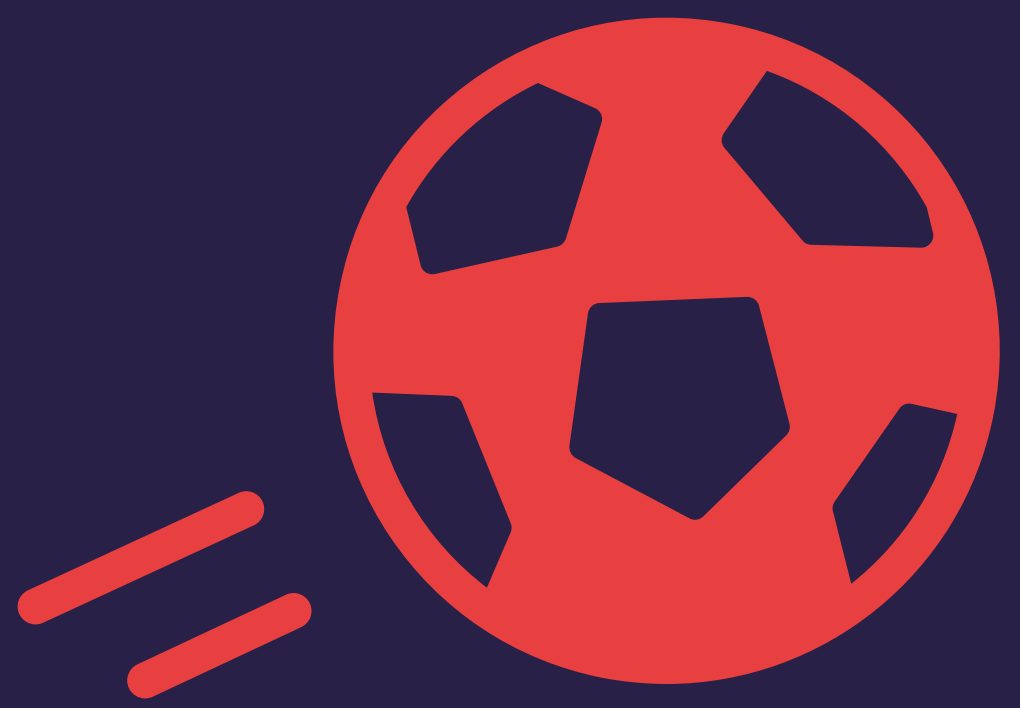





\section{Impact paragraph}

\section{This thesis}

The overall aim of this thesis was to contribute to the growing consensus that lifestyle factors affect brain health and dementia risk and to disseminate this knowledge to the general public. More specifically, this thesis aimed to validate the LIfestyle for BRAin health (LIBRA) index, a risk score consisting of twelve potentially modifiable risk and protective factors (such as smoking, hypertension, or physical inactivity), for cognitive outcomes (functioning, impairment and decline) and structural brain markers on MRI. In addition, we aimed to assess knowledge of dementia risk reduction in the general population and raise awareness on this matter using two proof-of-concept studies: a 10-month public health awareness campaign and the MijnBreincoach app.

The results of this thesis provide us with several insights. First, this thesis contributes to previous work showing that higher (i.e., unhealthier) LIBRA scores are associated with lower cognitive performance and more cognitive decline. Additionally, this thesis provides first evidence for higher LIBRA scores being associated with lower structural brain volumes and more white matter hyperintensities, an indication for neurodegeneration and cerebrovascular damage. These results suggest that LIBRA is a useful tool to identify (groups of) individuals at-risk for dementia and monitor room-for-improvement over time, for example in primary care or lifestyle intervention trials. Second, this thesis provides information on the level of awareness of dementia risk reduction in community-dwelling individuals in the province of Limburg, the Netherlands, and shows that most people were unaware of the potential of primary prevention of dementia. Considerable gaps in knowledge existed for the vascular risk factors of dementia, such as hypertension and high cholesterol. The good news is that most people were eager to learn more about this topic and most participants were positive about using eHealth to improve (knowledge on) brain health. We subsequently conducted a 10-month public health campaign to promote awareness in the Province of Limburg, the Netherlands, which resulted in an increase in recognition of two out of the three campaign themes ("eat healthy" and "exercise regularly"). Unfortunately, population-level awareness did not change over time. Yet, the individuals (in the post-campaign assessment) that stated to have heard of the campaign were more aware of dementia risk reduction than the individuals that had not heard of the campaign. This campaign only had limited resources to reach 
people and it is therefore recommended that future campaigns scale up to maximize exposure and engagement in the population. The MijnBreincoach app was investigated in another study. On the one side, this study showed us that most users rated the (concept of the) app positively and stated to have increased their knowledge on the topic. On the other side, this study uncovered technical issues of the app that caused users to drop-out and limited the use of several new functions of the app. Altogether, this study was designed as a proof-of-concept, aimed at identifying both the barriers and difficulties in the development of an mHealth tool for dementia risk reduction, as well as the advantages and gains, to improve our understanding of important factors that need to be considered in future studies. An elaboration on this, including optimizing the usability of the mHealth tool, was discussed in more detail in Chapter 6.

\section{Relevance: what does this thesis contribute to science and society?}

This thesis supports the increasing consensus that lifestyle affects dementia risk and that there is a need to inform the public about this matter. LIBRA is the only well-validated dementia risk score that focusses solely on the dementia risk fraction that is potentially modifiable through lifestyle interventions and optimized cardiovascular risk management. LIBRA could therefore be of added value in several research contexts, from epidemiological studies using LIBRA as a predictor variable for the risk of dementia and related conditions such as stroke, to lifestyle prevention trials and clinical trials using LIBRA as a surrogate outcome measure.

In 2018, we developed the MijnBreincoach app that uses LIBRA as a framework to identify room-for-lifestyle improvement and informs people about the relationship between lifestyle and brain health. MijnBreincoach was launched in March 2018, together with the 10-month public health awareness campaign in the Province of Limburg. With MijnBreincoach, we aim to bridge the gap between scientific research and society by offering this app free-of-charge to all Dutch inhabitants while doing research (with consent) on the anonymized usage tracking data. This provides us with the opportunity to raise awareness in the public about dementia risk reduction while at the same time fine-tuning the app based on direct user feedback.

To date, several national and international partners have adopted LIBRA, the public health campaign "We zijn zelf het medicijn" and MijnBreincoach. 
For example, all visitors of the Healthy Brain Ageing Clinic at the Brain and Mind Centre in Sydney, Australia answer questions to obtain information on all LIBRA factors. LIBRA will also be used in two large-scale Dutch projects that have been launched in 2021. The Dutch FINGER-NL intervention trial, in which participants will receive a high-intensity or low-intensity lifestyle intervention for a duration of 24 months, uses LIBRA as a surrogate outcome measure. The ABAORD project will adapt the current LIBRA to a clinical setting for people with subjective cognitive complaints or mild cognitive impairment. LIBRA is also being used to detect room for improvement during a multiple risk behaviour change intervention on dementia risk literacy, launched in 2021 at Macquarie University, Australia. Hopefully our campaign can serve as a blueprint for a nationwide campaign aimed at increasing awareness of dementia risk reduction. Two municipal health services (GGD West Brabant and GGD Haaglanden) in the Netherlands are currently running a locallyadapted dementia awareness campaign with the MijnBreincoach app and the design and materials of our campaign. The Danish Alzheimer's Association and the Norwegian National Advisory Unit on Ageing and Health will use the web portal version of MijnBreincoach, in which individuals can complete the LIBRA test to identify room-for-improvement (but does not provide subsequent informative daily notifications). Our survey to measure awareness of dementia risk reduction, which was based on the British Social Attitudes survey and assessed awareness on all twelve LIBRA factors (e.g., "Do you agree or disagree (on a 5-point Likert Scale) that hypertension increases the risk of getting dementia?") is being used in several countries, such as Belgium and France.

\section{Target group: who could benefit from this thesis?}

The results of this thesis could be of added value for researchers, clinicians and policy-makers working in the public health domain in general, or specifically in the field of dementia risk reduction. The lessons learned and recommendations for future (research) projects provided in this thesis could be of benefit to these professionals and hopefully lead to multidisciplinary collaborations across fields. The results of this thesis are also relevant to community-dwelling individuals, especially considering the growing number of people with dementia worldwide and the absence of treatment options.

\section{Activities: how to inform and involve the target group?}

We published all of our scientific work in open access journals that are freeof-charge available and we have reprinted the campaign results (Chapter 
5) in a Dutch scientific journal (Tijdschrift voor Gerontologie en Geriatrie) to facilitate collaborations within the Netherlands. Results of this thesis have been presented during the Alzheimer's Association International Conference in Los Angeles and the Alzheimer Europe Conference in The Hague, both in 2019.

A large part of the work in this thesis was aimed at disseminating scientific knowledge to the general public and, therefore, informing and involving this target group was in fact the essence of this thesis. We have used several strategies to inform the target group about dementia risk reduction, the awareness campaign and MijnBreincoach app. First, we strive to communicate our scientific results to the general public in lay-man's terms. During the campaign, we organized weekly educational events on dementia risk reduction throughout the Province of Limburg (e.g., lectures, workshops). We published a short booklet that provides information on the design, look-and-feel and results of the campaign and MijnBreincoach app, accompanied by attractive visuals. The booklet is free-of-charge available on request and can also be downloaded from our campaign website. The campaign results have been presented during a symposium for all "friends" of the campaign (more than 140 in total, e.g., national stakeholders, municipalities, health care centres, schools, supermarkets) at the provincial government (Gouvernement). The campaign friends received the short booklets during the symposium to distribute to their network. Our results were also presented during an educational event that is scheduled a few times a year in which researchers present their work in lay-man's terms to the general public ("Dialogen rond Dementie", organized by the Alzheimer Centre Limburg). With the help of several press releases, we have reached national and regional media (items in newspapers and on radio and TV). The MijnBreincoach app, which will remain available, will be further developed and has an increasing reach around the world, provides us with the opportunity to inform people about the potential of dementia risk reduction. The fact that the Dutch Ministry of Health, Welfare and Sport (Zorg van $\mathrm{Nu}$ ) and the municipal health services (GGD Appstore) have incorporated MijnBreincoach on their website on innovations in health care, will hopefully lead to a "snowball effect" in the Netherlands. Next to informing the general public, we also aimed to involve them in our research by organizing client panels in which people with dementia, their caregivers and people with a general interest in dementia provided us with feedback on research ideas, custom questionnaires and products, such as MijnBreincoach. 
Impact paragraph 



\section{List of publications}

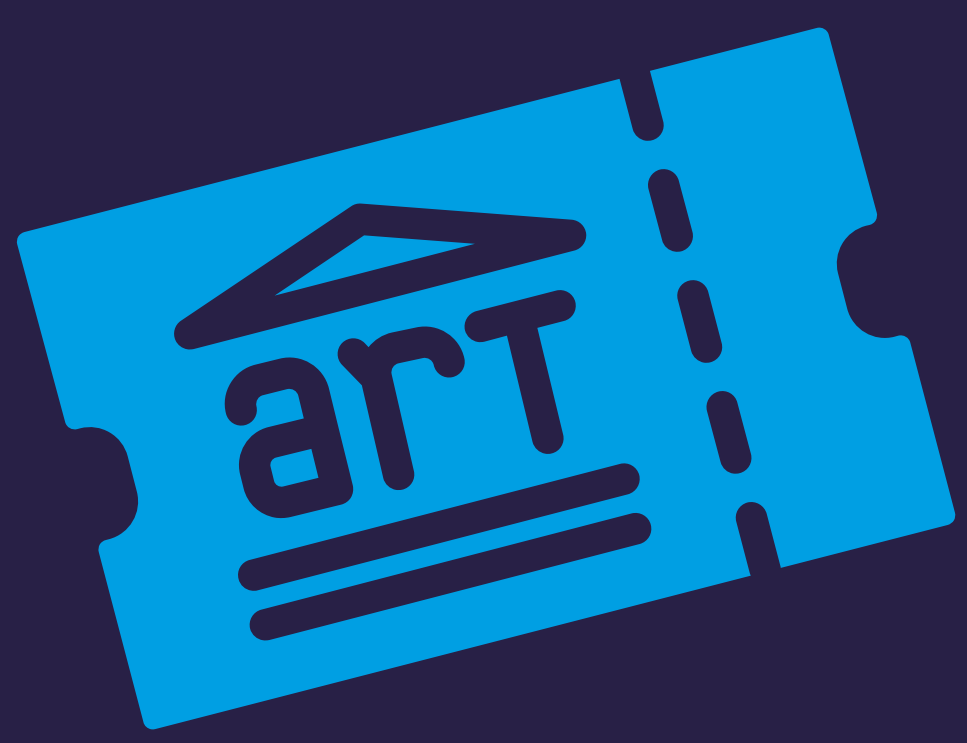





\section{List of publications}

\section{Thesis}

Heger I, Deckers K, van Boxtel M, de Vugt M, Hajema K, Verhey F, Köhler S. Dementia awareness and risk perception in middle-aged and older individuals: baseline results of the MijnBreincoach survey on the association between lifestyle and brain health. BMC Public Health. 2019 Jun 3;19(1):678. doi: 10.1186/s12889-019-7010-z.

Heger I, Köhler S, van Boxtel M, de Vugt M, Hajema K, Verhey F, Deckers K. Raising awareness for dementia risk reduction through a public health campaign: a pre-post study. BMJ Open. 2020 Nov 6;10(11):e041211. doi: 10.1136/bmjopen-2020-041211.

Heger IS, Deckers K, Schram MT, Stehouwer CDA, Dagnelie PC, van der Kallen CJH, Koster A, Eussen SJPM, Jansen JFA, Verhey FRJ, van Boxtel MPJ, Köhler S. Associations of the Lifestyle for Brain Health Index With Structural Brain Changes and Cognition: Results From the Maastricht Study. Neurology. 2021 Sep 28;97(13):e1300-e1312. doi: 10.1212/WNL.0000000000012572. Epub 2021 Aug 25. PMID: 34433680; PMCID: PMC8480401.

Heger I, Deckers K, van Boxtel M, de Vugt M, Hajema K, Verhey F, Oenema A, Köhler S. Appreciation of an mHealth tool to increase knowledge and beliefs and attitudes for dementia risk reduction: a pre-post proof-of-concept study. Journal of Medical Internet Research mHealth and uHealth, pending revisions.

Heger I, van Boxtel M, Deckers K, de Vugt M, Hajema K, Bosma H, Verhey F, Köhler S. Socioeconomic position, modifiable dementia risk and cognitive decline: results of 12-year the Maastricht Aging Study.

International Journal of Epidemiology, submitted.

\section{Other}

Van Asbroeck S, van Boxtel MPJ, Steyaert J, Köhler S, Heger I, de Vugt M, Verhey F, Deckers K. Increasing knowledge on dementia risk reduction in the general population: Results of a public awareness campaign. Preventive Medicine. 2021 Jun;147:106522. doi: 10.1016/j.ypmed.2021.106522. 
Heger I, Köhler S, van Boxtel M, de Vugt M, Hajema K, Verhey F, Deckers K. Een publiekscampagne om bewustwording over risicoreductie van dementie te verhogen: een pre-post studie. Tijdschrift voor Gerontologie en Geriatrie. 2021 Jun;52(2). doi: 10.36613/tgg.1875-6832/2021.02.01 
List of publications 



\section{Dankwoord}

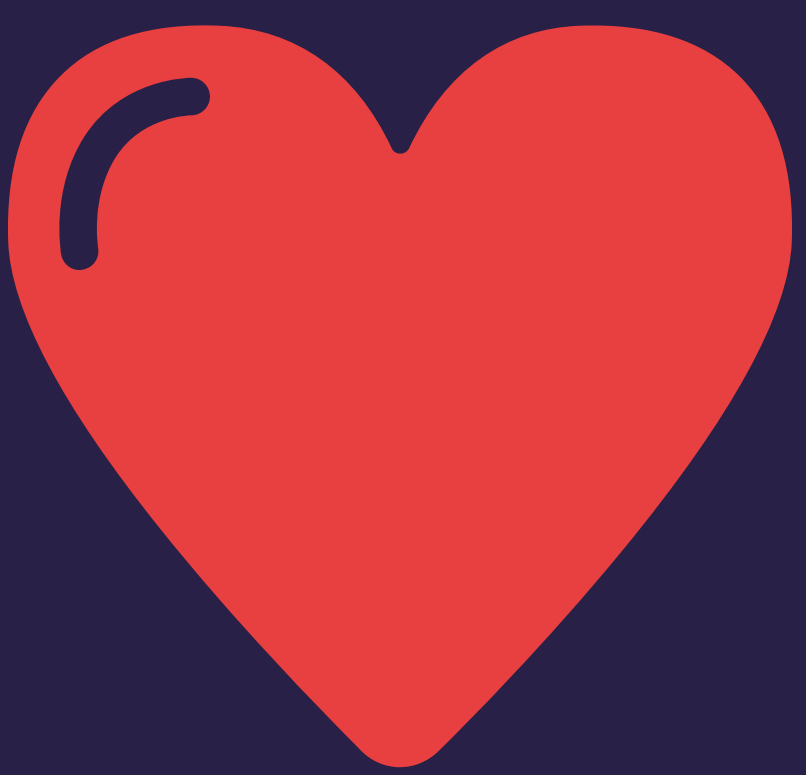





\section{Dankwoord}

Allereerst wil ik mijn promotieteam bedanken: Sebastian Köhler, Frans Verhey en Martin van Boxtel. Jullie fijne begeleiding, constructieve feedback (altijd startend met een complimentje!) en kritische blik hebben dit proefschrift gemaakt tot wat het is en daar ben ik jullie zeer dankbaar voor. Ik ben blij dat we de komende jaren blijven samenwerken.

Seb, bedankt voor al je wijze lessen. Ik heb de afgelopen vier jaar met fascinatie gekeken naar jouw kennis over en enthousiasme voor het werken met statistische modellen. Ik weet nog goed hoe we voor het eerst samen met Kay analyses zouden draaien voor De Maastricht Studie en jullie beiden helemaal verheugd waren om te zien wat eruit zou komen, alsof we een spannende film aan het kijken waren. Je staat op de afdeling niet voor niets bekend om je excellente kennis van statistiek, maar ik zou willen aanvullen dat jouw kracht ook ligt in onderwijs. Jouw rust en geduld zijn heel waardevol als docent en hebben er, naast je enthousiasme, voor gezorgd dat ook ik nu met plezier aan mijn analyses werk!

Frans, tijdens het sollicitatiegesprek zei je tegen me "het kunnen best vier pittige jaren gaan worden, maar we hebben wel een erg leuk team", waarna je me vol trots de (inmiddels oude) ACL-folder meegaf. Pittig was het op zijn tijd zeker, maar ik kijk vooral terug op vier leuke en leerzame jaren waarin ik me heb mogen ontwikkelen als onderzoeker onder jouw begeleiding. Bedankt voor je persoonlijke betrokkenheid tijdens de promotie overleggen en voor je toegewijde hulp tijdens de We Zijn Zelf Het Medicijn campagne, waarin je -ondanks je drukke agenda- altijd enthousiast de paarse ACL-jas aantrok en meehielp met het uitdelen van flyers en verzorgen van presentaties.

Martin, je hebt me altijd aangemoedigd om tijdens mijn $\mathrm{PhD}$ voldoende stil te staan bij mijn persoonlijke ontwikkeling en na te denken over welk pad ik in wil slaan na afloop van het traject en wat er voor nodig is om daar te komen. Ik ben je daar zeer dankbaar voor, want daardoor heb ik op een breed vlak cursussen kunnen volgen en weet ik nu beter waar mijn ambities liggen voor de toekomst. 
Aansluitend zou ik de overige twee leden van het MijnBreincoach team willen bedanken:

Kay, ondanks dat je officieel geen lid van mijn promotieteam bent, heb ik dat wel altijd zo ervaren. Je bent betrokken en altijd hard werkend. Ik vind het mooi om te zien hoe de preventie-onderzoekslijn jou nauw aan het hart ligt: jij vertelt "ons verhaal" altijd met verve. Of het nou op een groot symposium op het Gouvernement is, of bij de plaatselijke jeu de boules vereniging. Ik heb met veel plezier samen met jou de campagne gerund, crossend door de provincie in mijn Skoda Citigo (inclusief lekke band, waarbij alle campagneposters op de vluchtstrook uitgeladen moesten worden om bij de extra band te komen!). Erg leuk dat we blijven samenwerken de komende jaren. Heel veel geluk samen met Claudia, Cas en Bo.

Marjolein, we hebben tijdens mijn $\mathrm{PhD}$ met name in de eerste anderhalf jaar samengewerkt voor de campagne en de MijnBreincoach app. Ik heb veel bewondering voor jouw natuurlijke manier van presenteren en de wijze waarop je de kliniek en onderzoek weet te combineren. Ik ben erg blij dat we de komende jaren weer meer gaan samenwerken voor het ABOARD-project.

Graag zou ik de leden van de beoordelingscommissie willen bedanken voor het lezen en beoordelen van mijn proefschrift.

Ook zou ik alle participanten uit de studies in dit proefschrift hartelijk willen danken voor hun deelname. Het gaat hierbij om deelnemers uit De Maastricht Studie, MAAS, de twee steekproefmetingen in de provincie Limburg voor de bewustwordingscampagne en gebruikers van de MijnBreincoach app. Daarnaast wil ik alle coauteurs bedanken voor hun waardevolle feedback op de manuscripten.

Er zijn een aantal mensen die ik graag wil bedanken voor hun hulp bij het opzetten van de bewustwordingscampagne We Zijn Zelf Het Medicijn en de MijnBreincoach app.

Wat betreft de campagne wil ik allereerst alle campagnevrienden bedanken voor hun inzet. Zonder jullie was de campagne niet hetzelfde en niet even leuk geweest. Het enthousiasme waarmee jullie posters hebben opgehangen en activiteiten hebben georganiseerd is hartverwarmend. Alle collega's van 
Zuiderlicht bedankt voor het maken van het prachtige campagnemateriaal en de campagnewebsite. Extra dank voor Peggy van Sebillen en Eline Dekker voor jullie input en strategisch advies gedurende het campagnejaar, en voor Ruud Temmink en Emi Brok voor het vormgeven van dit proefschrift (erg mooi geworden!). Fania, we hebben samen meerdere campagne-klussen opgepakt en dat was altijd erg gezellig. Met name de opdracht voor het Technasium vond ik leuk om samen te doen. Ik hoop dat we nog lang blijven samenwerken! Ook wil ik Paula Snijders bedanken voor de hulp bij alle campagneactiviteiten.

Voor de ontwikkeling van de MijnBreincoach app wil ik allereerst graag de collega's van Betawerk en Sananet bedanken voor de hulp bij het ontwikkelen van de app. Daarnaast wil ik alle inhoudelijk experts bedanken voor het lezen en beoordelen van de notificaties uit de app. Anke Oenema, ik heb veel gehad aan jouw expertise op het gebied van gezondheidsbevordering en het ontwikkelen en evalueren van apps. Bedankt voor de vele uren aan begeleiding die je in het MijnBreincoach project hebt gestopt. Stephanie, ik heb vorig jaar met veel plezier samen met jou gewerkt aan de MijnBreincoach app. Bedankt voor al je goede werk, ik laat de app met een gerust hart achter in jouw handen! Leden van het cliëntpanel, jullie input was essentieel en zeer waardevol, waarvoor nog hartelijk dank.

Bedankt aan alle collega's van het Alzheimer Centrum Limburg en het Expertisecentrum Hersenletsel Limburg voor de fijne samenwerking, de gezellige werksfeer en de gesprekjes bij het koffiezetautomaat. Jammer genoeg hebben we elkaar de afgelopen periode wat minder gezien vanwege het vele thuiswerken en mijn zwangerschapsverlof. In het bijzonder wil ik Danielle en Els bedanken voor hun onmisbare hulp op de afdeling. Daarnaast wil ik Ron en Joost bedanken voor hun hulp bij de MijnBreincoach vragenlijsten en voor de vele teamviewer-sessies om mijn IT-problemen te verhelpen. Niels, Hannah, Leonie, Anouk en Linda, dank jullie wel voor jullie tips en tricks voor het afronden van een proefschrift, hier heb ik erg veel aan gehad. Jeroen, bedankt voor de gezelligheid die jij bracht op onze gezamelijke werkkamer.

Lieve Lieke, dank je wel voor je Brabantse nuchterheid en humor. Ik heb erg genoten van onze werkreizen richting Gent en Los Angeles (al moet ik dit misschien niet te hard roepen gezien mijn stelling 10). Wat bijzonder dat we elkaar vorig jaar februari tijdens een wandeling in Maastricht konden vertellen dat we allebei zwanger waren, en uiteindelijk met 4 dagen verschil zijn bevallen 
van Sophia en Daan. Ik wens jou, Rik en Daan alle goeds toe, en vooral veel rust en gezondheid om samen te genieten.

Lieve Julia, in vier jaar tijd zijn we van kamergenootjes bij het ACL goede vriendinnen geworden. Hoeveel kilometers wij inmiddels gewandeld hebben zou ik niet meer weten. Ik kijk altijd uit naar onze therapeutische rondjes over de Sint Pietersberg en door het Cannerbos. Onze vele zelfreflecties en psychoanalyses hebben volgens mij evenveel helpende inzichten gebracht als nieuwe problemen gecreëerd. Ik kom altijd met een glimlach op mijn gezicht thuis van onze rondjes.

Lieve Steef en lieve An, jullie zijn de liefste grote broer en kleine zus die ik me kan voorstellen. Hoe bijzonder (en bizar toevallig!) dan we in 12 maanden tijd alle drie een eerste kind hebben gekregen: Emily, Sophia en Julian. Ondanks de afstand zien we elkaar gelukkig regelmatig en dat voelt altijd als vanouds. Ik heb nu al zin in de weekendjes Recht samen met jullie, Stefanie, Sander en de kleintjes.

Lieve mama, bedankt voor jouw enthousiasme, onvoorwaardelijke steun en liefde. Samen met papa heb jij ons geleerd te genieten van het leven, trots op jezelf te zijn en dromen na te jagen. Ik kijk iedere keer weer op van jouw dappere houding in het leven en van jouw onuitputbare energie. Ik zal nooit vergeten hoe wij tijdens mijn verhuizing naar een nieuwe studentenkamer met zijn tweeën een stofzuiger en deel van mijn bed dwars door Maastricht hebben gedragen, met een pitstop op een terrasje op de Markt om even uit te blazen (met een hoop bekijks!). Dank je wel voor de lieve moeder die je voor me bent, en voor de lieve oma voor Sophia. Ik ben trots op je.

Lieve Rob, al meer dan tien jaar ben je mijn rots in de branding. Dank je wel voor je relativeringsvermogen en humor tijdens mijn stressmomentjes, voor je eerlijkheid, en natuurlijk vooral voor je uitmuntende Excel skills. Ik ben elke dag blij dat wij samen zijn, of we nou samen naar de sterrenhemel kijken in de Australische Snowy Mountains of langs de A2 een poepluier van Sophia verschonen. We vonden deze tekst zo passend voor de geboorte van Sophia, maar wat mij betreft geldt dit even goed voor jou: How wonderful life is, now you are in the world. 
Lieve, lieve Sophia. Je bent veel te klein om dit allemaal mee te maken. Toch vind ik het een bijzonder idee dat jij al lekker in mijn buik aan het trappelen was toen ik de laatste hoofdstukken van dit proefschrift schreef. Zo was je er toch al een beetje bij. Je hebt mijn leven in elk opzicht op zijn kop gezet en het is er alleen maar beter, mooier en betekenisvoller van geworden. Ik hou van je.

Lieve papa.

Wat ontzettend verdrietig dat je dit allemaal niet mee kan maken. Wat had je dit een mooie tijd gevonden, met de komst van drie kleinkinderen. Ik had Sophia zo graag aan je voorgesteld. Jouw humor, rust en liefde voor de natuur en Limburg zal ik haar zeker meegeven.

Jij was de eerste die de onderzoeker in mij zag. "Ik zie je zo voor me", zei je dan, "rijdend in een sportautootje met een mooi colbertje, op weg naar de universiteit" (soms wist ik niet zeker of je het over mij of over jezelf had!).

Ik mis je en ik denk aan je als ik op 24 maart de aula van de Minderbroedersberg in stap. In jouw geliefde Limburg.

Lieve papa, deze is voor jou. 



\section{Thesis defences of MHeNs}

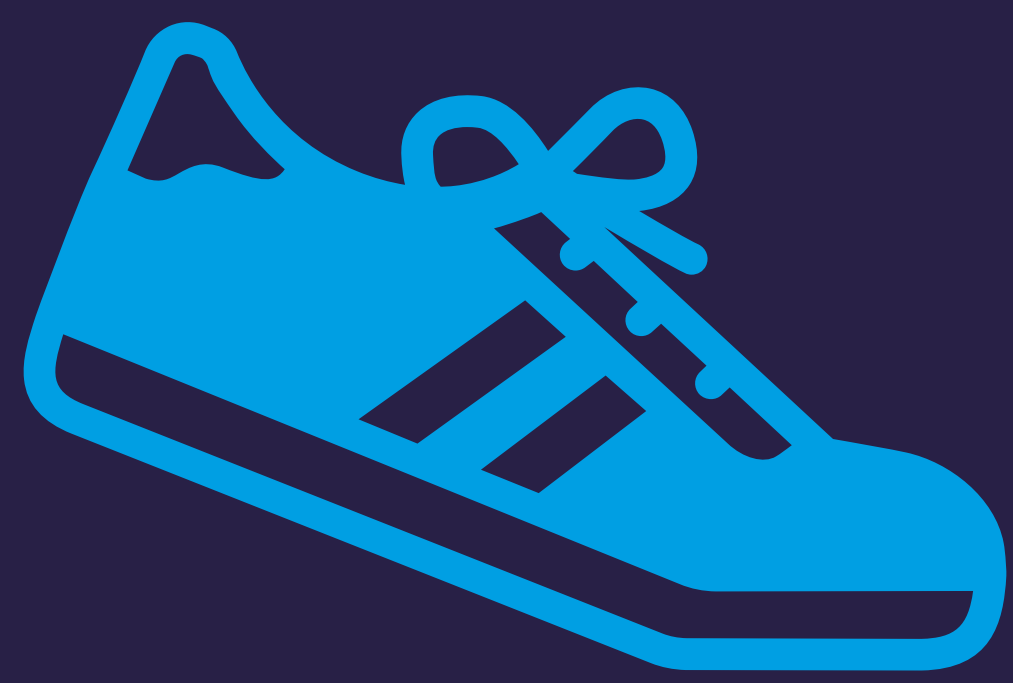





\section{Thesis defences from the School for Mental Health and Neuroscience (MHeNs)}

2021

Linda Pagen. Stress and Worry during cognitive aging: behavioral and neural correlates. Supervisors: Dr. Heidi I.l. Jacobs, Prof. Dr. Benedikt A. Poser. Co-supervisor: Prof. dr. Frans R.J. Verhey.

Christian Rauschenberg. Transdiagnostic Approaches to Mental Health: Linking adversity, cognition, candidate mechanisms, and novel digital interventions. Supervisor: Prof. Dr. J. van Os. Co-supervisor: Prof. Dr. U. Reininghaus.

Christian Nogales Calvo. Network Modules as Novel Molecular Disease Definitions for Precision Theranostics. Supervisors: Prof. Dr. Harald H.H.W. Schmidt, Dr. Ana I. Casas Guijarro.

Margot Heijmans. Track and treat Parkinson's disease using wearable sensors and MRI. Supervisor: Prof. Dr. Yasmin Temel. Cosupervisors: Dr. Pieter Kubben, Dr. Mark Kuijf.

Jessica Bruijel. Tired of being tired: Fatigue and sleep following traumatic brain injury. Supervisor: Prof. Dr. C.M. van Heugten. Cosupervisors: Dr. A. Vermeeren, Dr. S.Z. Stapert.

Naomi Daniëls. Bringing experiencesampling technology to family medicine: Feasibility, usability and lessons-learned. Supervisors: Prof. dr. P.A.E.G. Delespaul, Prof. dr. A.J. Beurskens. Co-supervisor: Dr. M.A. van Bokhoven.

Soraya Jonker. Safety and Efficacy of Intraocular Lenses in Cataract - and Refractive Surgery. Supervisor: Prof. Dr. R.M.M. A. Nuijts. Co-supervisors: Dr. N.J.C. Bauer, Dr. T.T.J.M. Berendschot.

Ana Maria Alzate Sànchez. Microelectrode recordings for deep brain stimulation: Patient specific variables yields fundamental and clinical insights into the human basal ganglia. Supervisor: Prof. Dr. Y. Temel. Cosupervisors: Dr. M.L.F. Janssen, Dr. M.J. Roberts.

Paula Bartholomeus. ReAttach - A transdiagnostic intervention for adults and children with mental health problems. Supervisor: Prof. Dr. T.A.M.J. van Amelsvoort. Co-supervisors: Prof. Dr. M. Fitzgerald, Prof. Dr. D. Marazziti.

Rik Schalbroeck. Orderly chaos: social defeat as a risk factor for psychosis in autism. Supervisors: Prof. Dr. J.P.Selten, Prof. Dr. L.F. de Geus-Oei, Prof. Dr. J. Booij.

Renzo Riemens. Neuroepigenomics in Alzheimer's disease: The single cell ADds. Supervisors: Prof. dr. D. van den Hove, Prof. dr. B. Rutten, Prof. dr. T. Haaf. Co-Supervisor: Dr. 
G. Kenis, Prof. dr. C. Förster, Prof. Dr. K.P. Lesch.

Vera Marsman-Bonekamp. Beyond dis-ease and dis-order - Exploring the long-lasting impact of childhood adversity in relation to mental health. Supervisor: Prof. Dr. J.J. van Os. Co-supervisor: Dr. H.B. Lousberg.

Kim van der Linden. Stress, anxiety and psychotic experiences in adults with autism spectrum disorder; and oberservational study in the context of daily life. Supervisors: Prof. Dr. M.C. Marcelis, Prof. Dr. T.A.M.J. van Amelsvoort. Co-supervisor: Dr. C.J.P. Simons.

Jeroen Habets. Prediction and real-life monitoring of DBS motor response in Parkinson's disease. Supervisor: Prof. dr. Y. Temel. Co-supervisor: Dr. P. Kubben, Dr. M. Kuijf.

Mohammed Alahmari. Radiological and radio-therapeutic nuances in skull base tumours. Supervisors: Prof. Dr. Y. Temel, Prof. Dr. Ir. F.J.W. Verhaegen. Co-supervisor: Dr. D. Eekers.

Michaël Veldeman. Diagnosis and treatment of early and delayed cerebral injury after aneurysmal subarachnoid hemorrhage. Supervisors: Prof. Dr. Y. Temel, Prof. Dr. H. Clusmann. Co-supervisor: Dr. R. Haeren.

Siyu Wu. Modulation of myelin phagocytosis by means of anti-inflammatory treatment as a therapy of spinal injury. Supervisors: Prof. Dr. B.W.W. Kramer, Dr. J. Mey.
Wouter Hubens. Glaucoma biomarkers in aqueous humor and blood. Supervisor: Prof. Dr. C.A.B. Webers. Co-supervisor: Dr. T.G.M.F. Gorgels.

Sjors van de Weijer. Digital technologyenabled home health care - Gamification in online cognitive therapies for Parkinson's disease. Supervisor: Prof. Dr. B.R. Bloem. Cosupervisors: Dr. A.A. Duits, Dr. M.L. Kuijf, Dr. N.M. de Vries.

Lisanne Canjels. Morphological and functional Magnetic Resonance Imaging at ultra-high field. Supervisors: Dr. J.F.A. Jansen, Prof. Dr. Ir. W.H. Backes, Prof. Dr. A.P. Aldenkamp. Co-supervisor: Dr. C. GhosseinDoha.

Yentl van der Zee. Novel Insights into the Neurophysiological and Epigenetic Changes in Major Depressive Disorder. Supervisor: Prof. Dr. B.P.F Rutten. Co-supervisors: Dr. O. Issler, Dr. L. de Nijs, Dr. L.M.T. Eijssen.

Judith Lionarons. Nonmotor comorbidities and somatic manifestations of Duchenne Muscular Dystrophy. Supervisor: Prof. Dr. C.G. Faber. Co-supervisors: Dr. G. Hoogland, Dr. J.G.M. Hendriksen, Dr. S. Klinkenberg.

Gusta van Zwieten. Silencing neural symphonies with deep brain stimulation. Supervisors: Prof. Dr. Y. Temel, Prof. Dr. R.J. Stokroos. Co-supervisor: Dr. M.L.F. Janssen.

Shuo Zhang. A dark field illumination probe linked to Raman spectroscopy for 
non-invasivety etermination of ocular biomarkers. Supervisors: Prof. Dr. C.A.B. Webers, Dr. T.T.J.M. Berendschot. Cosupervisor: Dr. R.J. Erckens.

Ahmed Hassan. FAIR and bias-free network modules for mechanism-based disease redefinitions. Supervisors: Prof. Dr. H.H.H.W. Schmidt, Prof. Dr. M.J. Dumontier.

Anouk Geraets. Biological determinants of depression, the role of cerebral damage, microvascular dysfunction, and hyperglycemia: a polulationbased approach. Supervisors: Dr. M.T. Schram, Prof. Dr. F.R.J. Verhey. Co-supervisor: Dr. S. Köhler.

Le Guo. No, They Didn't? Oh, They Did!; Advancing Insights on Social Norm Interventions in Consumer Financial Decision-Making. Supervisor: Prof. Dr. H.J.M. Smeets. Co-supervisors: Dr. F.H.J. van Tienen, Dr. M. Gerards.

Yvonne van der Zalm. An inquiry into various aspects of clozapine use: prescription, monitoring and mortality. Supervisor: Prof. Dr. J.P. Selten, Prof. Dr. I.E. Sommer. Co-supervisors: Dr. P.F.J. Schulte, Dr. F. Termorshuizen.

Onur Alptekin. Methodological aspects of deep brain stimulation: the untold story behind DBS surgery. Supervisor: Prof. Dr. Y. Temel. Co-supervisors: Dr. E. Kocabicak, L. Ackermans.
Elaine Schepers. The role of white noise speech illusions in indicating risk for psychotic disorders. Supervisor: Prof. Dr. J.J. van Os. Co-supervisor: Dr. R. Lousberg.

Christian Bertens. Development of a noninvasive ocular drug delivery device. Supervisor: Prof. Dr. R.M.M.A. Nuijts. Cosupervisors: Dr. M. Gijs, Dr. F.J.H.M. van den Biggelaar.

Remco Santegoeds. A journey of skull base chordoma: where imaging meets molecular biology. Supervisor: Prof. Dr. Y. Temel. Cosupervisors: Dr. L. Jacobi-Postma, Dr. D. Eekers.

Inge Verheggen. Imaging blood-brain barrier function in aging. Supervisors: Prof. Dr. W.H. Backes, Prof. Dr. F.R.J. Verhey. Co-supervisor: Dr. A. Jahanshahianvar.

Douwe van der Heide. On the assessment of symptom validity in refugee mental health. Supervisors: Prof. Dr. H.L.G.J. Merckelbach, Prof. Dr. P.N. van Harten.

Gowoon Son. Olfactory system pathology in Alzheimer's disease: evidences from rodent and human studies. Supervisors: Prof. dr. H.W.M. Steinbusch, Prof. Dr. C. Moon. Cosupervisor: Dr. A. Jahanshahianvar

Markos Xenakis. Molecular complexity of voltage-gated sodium channels; theory and applications in mutation-response prediction. Supervisor: Prof. Dr. H.J.M. Smeets. Co-supervisors: Dr. P.J. Lindsey, Dr. R.L. Westra. 
Alix Thomson. From Micro to Macro: Unravelling the Underlying Mechanisms of Transcranial Magnetic Stimulation (TMS). Supervisor: Prof. A.T. Sack. Co-supervisors: Dr. T.A. de Graaf, Dr. T. Schuhmann, Dr. G.R.L. Kenis.

Ozan Cinar. Combining In formation: Model Selection in Meta-Analysis and Methods for Combining Correlated p-Values. Supervisor: Dr. W. Viechtbauer. Co-supervisors: Dr. I.S. Gülöksüz.

Anne Koopmans. CYP2D6 and CYP2C19 genotyping in psychiatry - Bridging the gap between practice and lab. Supervisors: Prof. Dr. P.N. van Harten, Prof. Dr. H.W.Hoek. Cosupervisor: Dr. D.J. Vinkers.

Ashwin Mohan. Retinal oximetry in health and disease. Supervisor: Prof. C.A.B. Webers. Co-supervisors: Dr. T.T.J.M. Brendschot, Dr. R. Shetty.

Danique Hellebrekers. Neurocognition and behaviour: diagnostic work-up and interventions in Duchenne and Becker muscular dystrophy. Supervisors: Prof. J.S.H. Vles, Dr. J.G.M. Hendriksen. Co-supervisor: Dr. S. Klinkenberg.

Milaine Roet. Modulating microcircuits in depression. Supervisor: Prof. Y. Temel. Cosupervisor: Dr. A. Jahanshahianvar.
Julia van Tuijl. Post-stroke epilepsy. Supervisor: Prof. A.P. Aldenkamp. Cosupervisors: Dr. R.P.W. Rouhl, Dr. E.P.M. van Raak.

Hans de Munter. The patient's own bone marrow-derived stromal cells: disease modifiers in (neuro) degenerative disorders. Supervisors: Prof. B.W.W. Kramer, Prof. E.CH Wolters. Co-supervisors: Dr. T. Strekalowa, Dr. J. Mey.

Sophie Leijdesdorff. Ain't no mountain high enough - How to improve access to youth mental health care. Supervisors: Prof. T.A.M.J. van Amelsvoort, Prof. A. Popma. Cosupervisor: Dr. R.M.C. Klaassen.

Laura Vergoossen. Brain Network Alterations due to Cardiometabolic Risk Factors; Insights from Population Magnetic Resonance Imaging. Supervisor: Prof. W.H. Backes. Co-supervisors: Dr. J.F.A. Jansen, Dr. M.T. Schram.

Ranjana J. Jairam. Sacral nerve stimulation for lower urinary tract dysfunctions: towards better outcome. Supervisors: Prof. Ph.E.V.A. Van Kerrebroeck, Prof. G.A. van Koeveringe. Co-supervisor: Dr. D.M.J. Vrijens.

Luiz Kae Sales Kanazawa. The antimanic-like effects of andrographolide and quercetin. Supervisors: Prof. J. Prickaerts. Co-supervisor: Dr. Roberto Andreatini. 
Maria Ferrarac. Early Intervention in Psychosis A data-driven population health approach to reduce the duration of untreated psychosis. Supervisors: Dr. S. Gölöksüz, Prof. J.J. van Os. Co-supervisor: Prof. V.H. Srihari.

Talakad Narasappa Sathyaprabha. Cardiovascular autonomic regulation in health and neurological disorders. Supervisors: Pof. B.W.W. Kramer, Prof. H.W.M. Steinbusch. Co-supervisor: Dr. T.R. Raju.

Raoul Stevens. Unboxing the Brain; Development of Technologies for NonInvasive Assessment of Cerebral Pathologies. Supervisor: Prof. T. Delhaas, Prof. W.H. Mess. Co-supervisor: Dr. W. Huberts.

Clara Snijders. Post-traumatic stress disorder epigenetic signatures of differential susceptibility to combat trauma. Supervisor: Prof. B.P.F. Rutten. Co-supervisors: Dr. L. de Nijs, Dr. G. Kenis.

Thesis defences from MHeNs from previous years can be found via the following link: https://mhens.mumc. maastrichtuniversity.nl/ node/ 14680 

Author information 



\section{Author information}

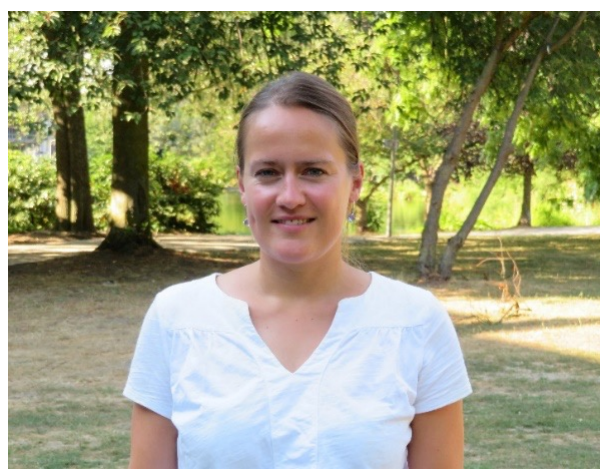

Irene Heger was born on 31 October 1987 in Voorburg. In 2006, she graduated from the Haags Montessori Lyceum and used the following year to follow an Academic English Language Course in Sydney and travel through Australia. She started her bachelor's degree in Health Science at Maastricht University in 2007 and subsequently started her two-year master's degree in Medical Psychology in 2011 at Tilburg University. In the final year of her degree, she conducted a 12-month clinical internship at the department of Medical Psychology at the TweeSteden Ziekenhuis in Tilburg. After finishing her master thesis on psychological problems in patients with a delirium at the geriatric ward of the hospital and completing her degree, she continued working at the TweeSteden Ziekenhuis as a medical psychologist and gained experience in bariatric care, cardiac rehabilitation and at the memory clinic. In 2014, she moved from Tilburg to The Hague and started working as a psychologist at the Dutch Obesity Clinic. Her work consisted of psychological screenings, individual counselling and group counselling for patients receiving bariatric surgery, all in a multidisciplinary setting. In October 2017, she moved to Maastricht and started working as a PhD-student at the Alzheimer Centre Limburg (Department of Psychiatry and Neuropsychology) of Maastricht University. During her $\mathrm{PhD}$, she worked in the MijnBreincoach-team, which organized a public health campaign and developed the MijnBreincoach mobile app. Irene is currently working as a postdoctoral researcher at the Alzheimer Centre Limburg.

Irene Heger is geboren op 31 oktober 1987 in Voorburg. In 2006 behaalde ze haar middelbareschooldiploma aan het Haags Montessori Lyceum. In het daaropvolgende jaar heeft ze een taalcursus wetenschappelijk engels gevolgd in Sydney en door Australië gereisd. In 2007 startte ze met haar bacheloropleiding Gezondheidswetenschappen aan de Universiteit van Maastricht, om vervolgens in 2011 te starten met de tweejarige masteropleiding Medische Psychologie aan de Universiteit van Tilburg. In het laatste jaar van deze opleiding heeft ze een 12-maanden durende klinische stage doorlopen op de afdeling Medische Psychologie van het TweeSteden Ziekenhuis te Tilburg. Na het afronden 
van haar masterthesis over psychologische problematiek bij patiënten met een delier op de afdeling geriatrie van het ziekenhuis en het behalen van haar diploma, bleef ze werken bij het TweeSteden Ziekenhuis als medisch psycholoog. Hier heeft ze ervaring opgedaan binnen de bariatrische zorg, de hartrevalidatie en de geheugenpoli. In 2014 verhuisde ze van Tilburg naar Den Haag en is ze gaan werken als psycholoog bij de Nederlandse Obesitas Kliniek. Haar werkzaamheden bestonden uit het uitvoeren van psychologische screenings en individuele en groepsbegeleiding in een multidisciplinaire setting bij patiënten die een bariatrische ingreep ondergaan. Ze verhuisde in oktober 2017 naar Maastricht, waar zij werkzaam werd als promovendus bij het Alzheimer Centrum Limburg (afdeling Psychiatrie en Neuropsychologie) van de Universiteit van Maastricht. Gedurende haar PhD-traject werkte ze in het MijnBreincoach-team, welke een publiekscampagne organiseerde en de MijnBreincoach app ontwikkelde. Momenteel is Irene werkzaam als postdoctoraal onderzoeker bij het Alzheimer Centrum Limburg. 

